\title{
The Architecture Analysis \& Design Language (AADL): An Introduction
}

\author{
Peter H. Feiler \\ David P. Gluch \\ John J. Hudak
}

February 2006

Performance-Critical Systems 
This work is sponsored by the U.S. Department of Defense.

The Software Engineering Institute is a federally funded research and development center sponsored by the U.S. Department of Defense.

Copyright 2006 Carnegie Mellon University.

NO WARRANTY

THIS CARNEGIE MELLON UNIVERSITY AND SOFTWARE ENGINEERING INSTITUTE MATERIAL IS FURNISHED ON AN "AS-IS" BASIS. CARNEGIE MELLON UNIVERSITY MAKES NO WARRANTIES OF ANY KIND, EITHER EXPRESSED OR IMPLIED, AS TO ANY MATTER INCLUDING, BUT NOT LIMITED TO, WARRANTY OF FITNESS FOR PURPOSE OR MERCHANTABILITY, EXCLUSIVITY, OR RESULTS OBTAINED FROM USE OF THE MATERIAL. CARNEGIE MELLON UNIVERSITY DOES NOT MAKE ANY WARRANTY OF ANY KIND WITH RESPECT TO FREEDOM FROM PATENT, TRADEMARK, OR COPYRIGHT INFRINGEMENT.

Use of any trademarks in this report is not intended in any way to infringe on the rights of the trademark holder.

Internal use. Permission to reproduce this document and to prepare derivative works from this document for internal use is granted, provided the copyright and "No Warranty" statements are included with all reproductions and derivative works.

External use. Requests for permission to reproduce this document or prepare derivative works of this document for external and commercial use should be addressed to the SEI Licensing Agent.

This work was created in the performance of Federal Government Contract Number FA8721-05-C-0003 with Carnegie Mellon University for the operation of the Software Engineering Institute, a federally funded research and development center. The Government of the United States has a royalty-free government-purpose license to use, duplicate, or disclose the work, in whole or in part and in any manner, and to have or permit others to do so, for government purposes pursuant to the copyright license under the clause at 252.227-7013.

For information about purchasing paper copies of SEI reports, please visit the publications portion of our Web site (http://www.sei.cmu.edu/publications/pubweb.html). 


\section{Contents}

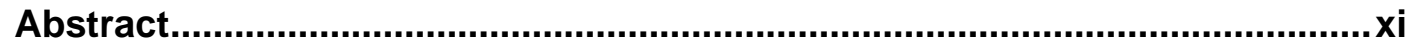

1 Introduction ..................................................................................... 1

1.1 Document Summary ............................................................... 1

1.2 Reader's Guide to Technical Interests ........................................ 2

1.3 Conventions Used in this Document............................................... 3

2 SAE AADL Overview.............................................................................. 4

2.1 Abstraction of Components ....................................................... 4

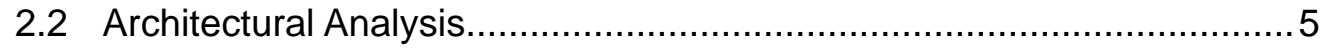

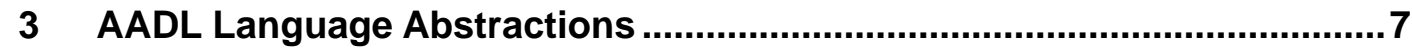

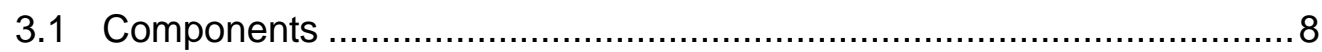

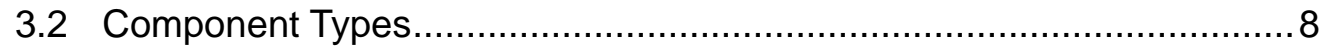

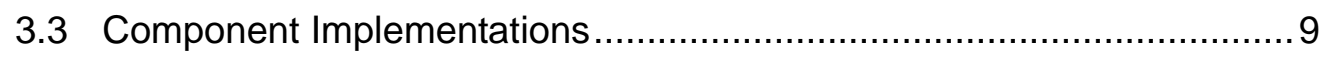

3.4 Packages, Property Sets, and Annexes .................................... 10

4 AADL System Models and Specifications ............................................. 11

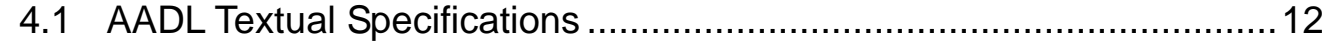

4.2 Graphical Representations ................................................... 13

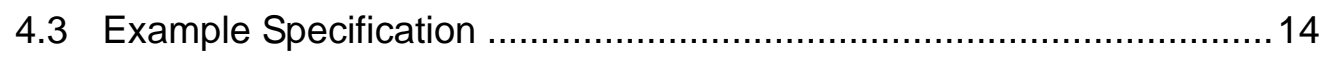

4.4 Type Declarations ................................................................. 16

4.5 Implementation Declarations ............................................... 18

4.6 Package Declarations .......................................................... 19

4.7 Property Set Declarations ................................................... 20

4.8 Annex Library Declarations.................................................... 20

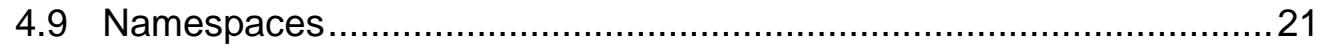

4.10 Partial Specifications ............................................................. 21

4.11 Extends, Refines, and Partial Specification .................................. 21

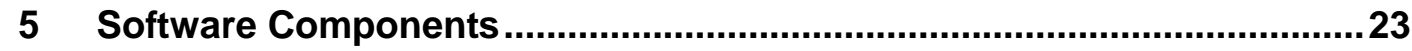

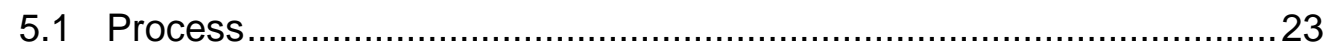

5.1 .1 Textual Representation .............................................. 23 
5.1.2 Graphical Repesenation ............................................... 24

5.1 .3 Properties .................................................................. 25

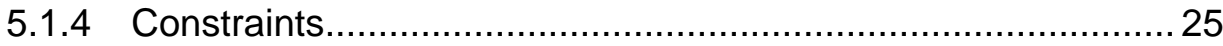

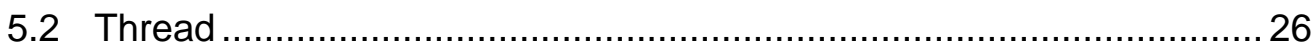

5.2.1 Textual Representation............................................... 26

5.2.2 Graphical Representation .......................................... 27

5.2.3 Thread Execution........................................................ 28

5.2 .4 Properties .................................................................. 29

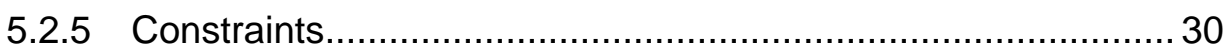

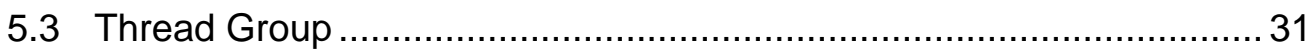

5.3.1 Textual Representation............................................... 31

5.3.2 Graphical Representation ............................................. 32

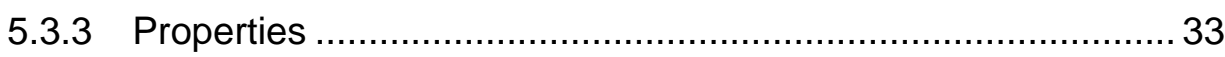

5.3.4 Constraints...................................................................... 33

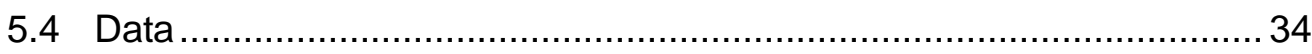

5.4.1 Textual Representation.................................................. 34

5.4 .2 Graphical Representation ............................................ 36

5.4 .3 Properties ........................................................................ 36

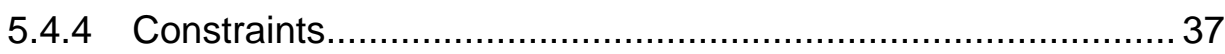

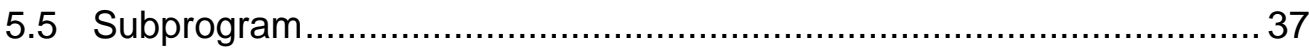

5.5 .1 Textual Representation........................................... 38

5.5.2 Graphical Representation ................................................ 38

5.5 .3 Properties .............................................................. 40

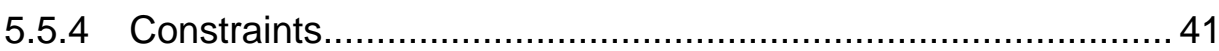

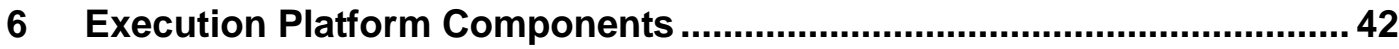

6.1 Processor ............................................................................... 42

6.1.1 Textual and Graphical Representations .............................. 43

6.1 .2 Properties .................................................................... 44

6.1.3 Constraints..................................................................... 44

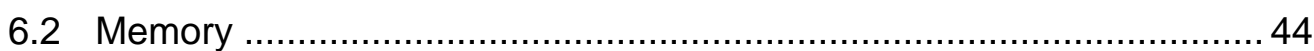

6.2.1 Textual and Graphical Representations ............................. 45

6.2 .2 Properties ....................................................................... 46

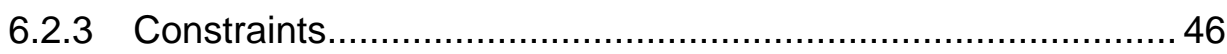

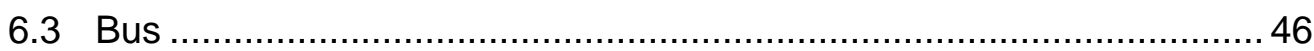

6.3.1 Textual and Graphical Representations ........................... 47

6.3.2 Properties ................................................................... 47

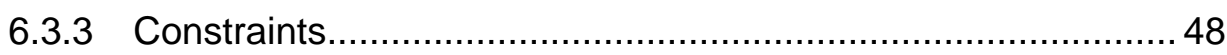

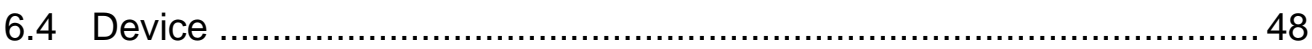

6.4.1 Textual and Graphical Representations ............................ 48

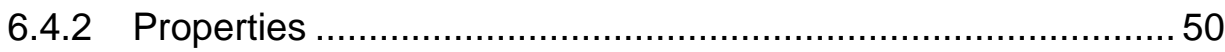

6.4.3 Constraints................................................................. 51 


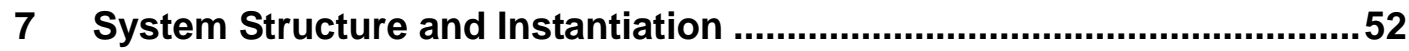

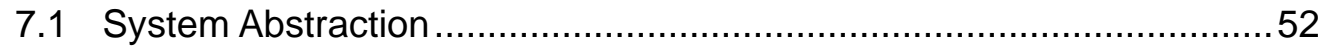

7.1.1 Textual and Graphical Representations.................................... 52

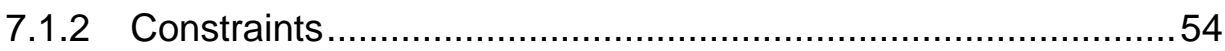

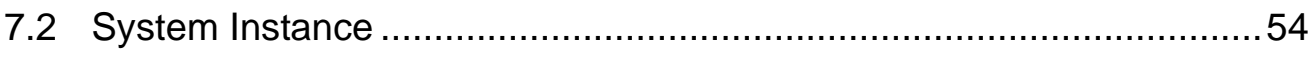

7.3 Binding to Execution Platform Components ……….............................55

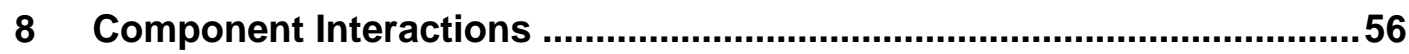

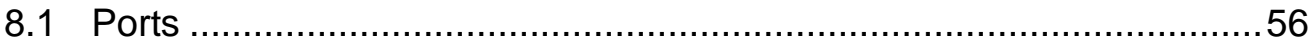

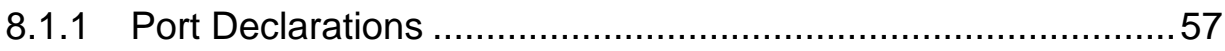

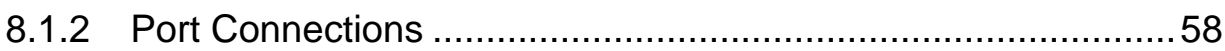

8.1.3 Connections in System Instance Models .................................60

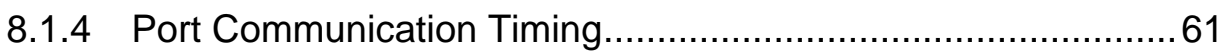

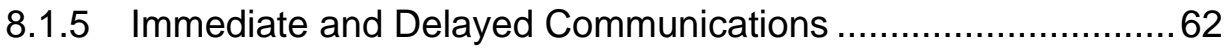

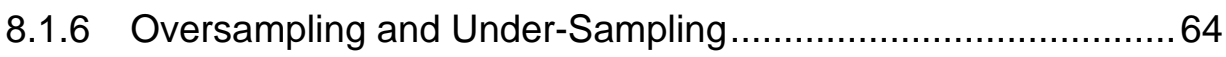

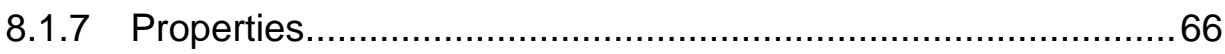

8.1.8 Port and Port Connection Constraints ......................................67

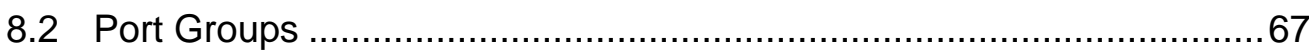

8.2.1 Port Groups and Port Group Type Declarations .......................68

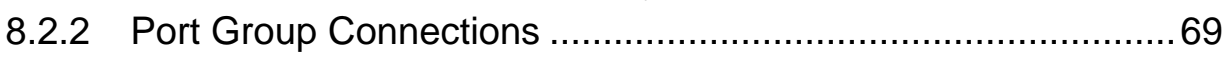

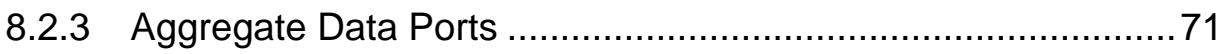

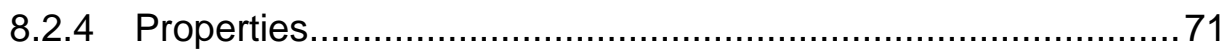

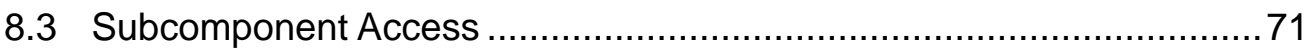

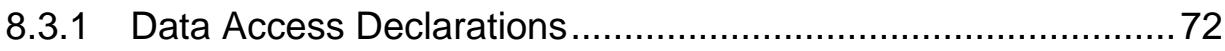

8.3.2 Data Access Connections .......................................................... 72

8.3.3 Bus Access and Bus Access Connections ……………......... 74

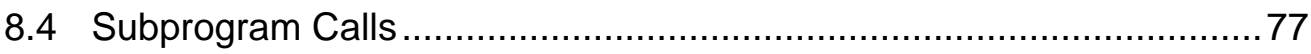

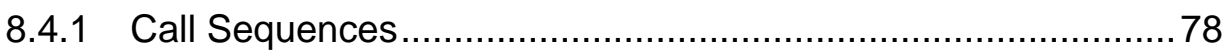

8.4.2 Remote Calls ................................................................ 79

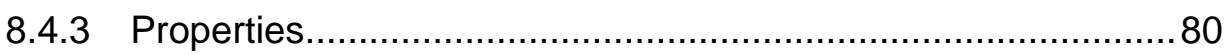

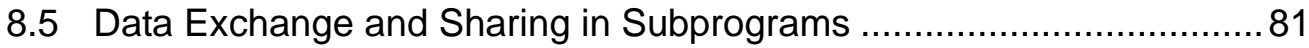

8.5.1 Data Exchange by Value: Parameters and Connections ........... 81

8.5.2 Data Passing by Reference and Global Data .......................... 82

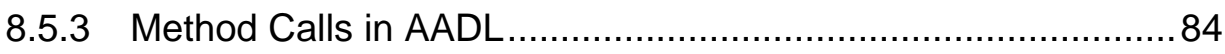

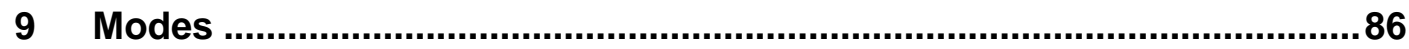

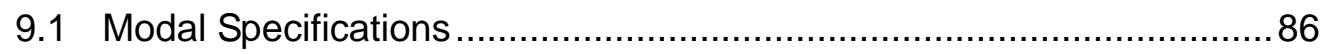

9.1.1 Modal Configurations of Subcomponents and Connections ......86

9.1.2 Modal Configurations of Call Sequences................................. 89

9.1.3 Mode-Specific Properties...................................................... 90

10 Flows

CMU/SEI-2006-TN-011 


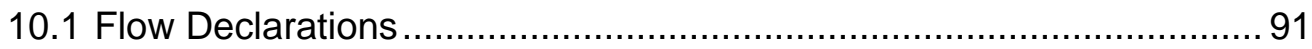

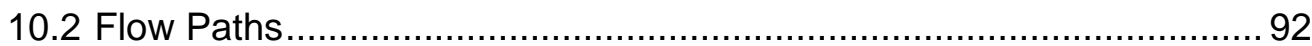

10.2.1 Flow Path through a Component ..................................... 92

10.2.2 End-to-End Flow within a Component ................................. 93

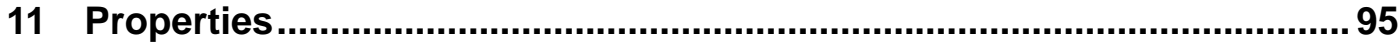

11.1 Property Declarations .................................................................. 95

11.2 Assigning Property Values......................................................... 96

11.2.1 Basic Property Associations ............................................... 96

11.2.2 Contained Property Associations........................................ 97

11.2.3 Inherited Property Associations ............................................ 100

11.2.4 Mode or Binding Specific Property Associations .................. 101

11.2.5 Property Values ................................................................ 102

11.3 Defining New Properties.......................................................... 103

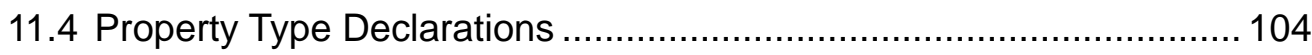

11.5 Property Name Declarations .................................................... 105

11.6 Property Constant Declarations................................................. 106

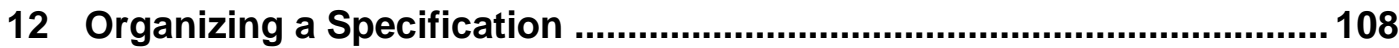

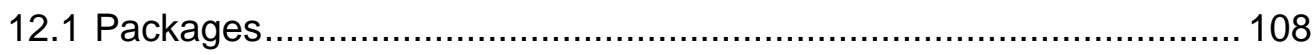

12.2 Design Patterns ..................................................................... 111

12.2.1 Type Extensions ...................................................... 111

12.2.2 Refinements within Implementations ................................ 112

12.2.3 Implementation Extensions.......................................... 113

12.2.4 Example Design Patterns ............................................ 115

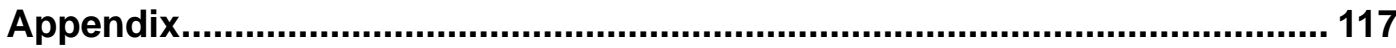

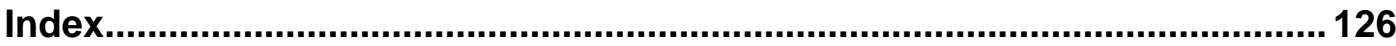

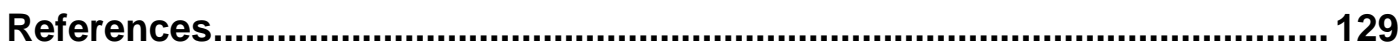




\section{List of Figures}

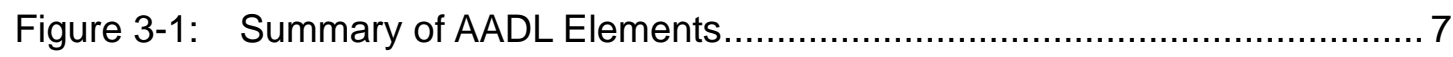

Figure 3-2: Subclauses of a Type Declaration ........................................... 8

Figure 3-3: Subclauses of an Implementation Declaration............................... 9

Figure 4-1: AADL Representations...................................................... 11

Figure 4-2: AADL Graphical Notation .................................................. 13

Figure 5-1: Graphical Representation of a Sample Process .............................. 25

Figure 5-2: Thread Execution State Machine ............................................ 29

Figure 5-3: A Sample Thread Group Graphical Representation........................... 32

Figure 5-4: Sample Data Component Graphical Representations ........................ 36

Figure 5-5: Subprogram Graphical Representation ...................................... 39

Figure 6-1: A Device as Part of the Physical Hardware .................................. 50

Figure 6-2: A Device as Part of the Application System ................................. 50

Figure 6-3: A Device as Part of the Controlled Environment ............................... 50

Figure 8-1: Port Graphical Representations.............................................. 57

Figure 8-2: A Semantic Connection between Thread Instances ......................... 61

Figure 8-3: A Connection Instance in a Partially Specified System Instance Model........................................................ 61

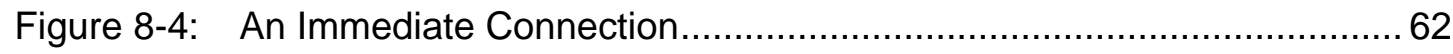

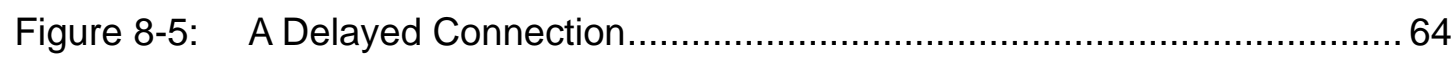

Figure 8-6: Oversampling with Delayed Connections ................................... 65 


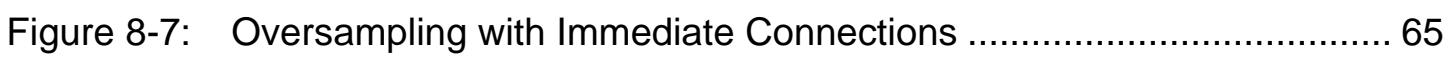

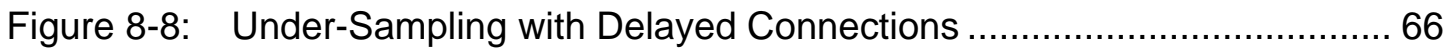

Figure 8-9: $\quad$ Under-Sampling with Immediate Connections................................. 66

Figure 8-10: Graphical Representations of Port Groups ................................... 69

Figure 8-11: Sample Port Group Connections ................................................. 70

Figure 11-1: Contained Property: Allowed_Processor_Binding ….................... 100

Figure 12-1: 3-Way Voting Lane Component ....................................................115

Figure 12-2: Generic N-Way Voting Lanes Type-Implementation Pairs .................115

Figure 12-3: Specialized Extension and Refinement ..................................116 


\section{List of Tables}

Table 1-1: $\quad$ Summary of Content in this Document ...................................... 1

Table 1-2: $\quad$ Technical Interests and Relevant Sections in this Document................ 2

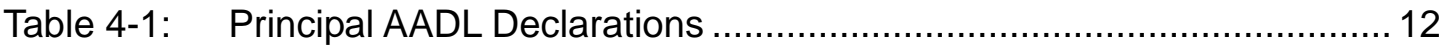

Table 4-2: $\quad$ A Simplified Example of an AADL Specification ............................... 15

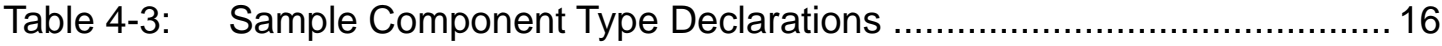

Table 4-4: Component Implementation Declarations ........................................ 19

Table 4-5: Example Packages …................................................................. 20

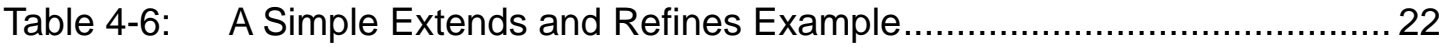

Table 5-1: $\quad$ Textual Representation of a Sample Process .................................. 24

Table 5-2: $\quad$ Summary of Permitted Process Declarations ................................. 26

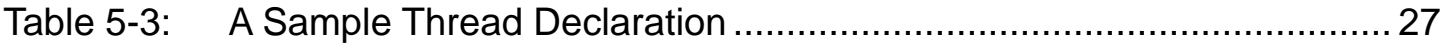

Table 5-4: $\quad$ A Sample Thread Implementation with One Subcomponent ............... 28

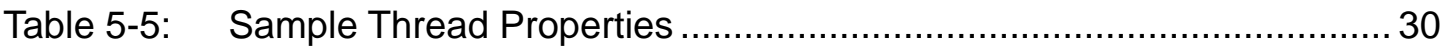

Table 5-6: $\quad$ Summary of Permitted Thread Subclause Declarations ..................... 30

Table 5-7: $\quad$ A Sample Thread Group AADL Textual Specification.......................... 31

Table 5-8: $\quad$ Elements of a Thread Group Component ........................................ 33

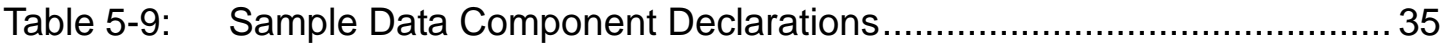

Table 5-10: Legal Elements of Data Type and Implementation Declarations .......... 37

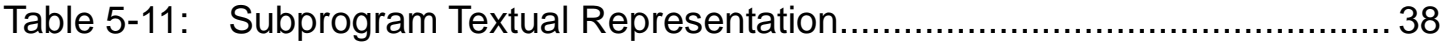


Table 5-12: Example Textual and Graphical Subroutine Declarations

Table 5-13: Restrictions on Subprogram Declarations ...................................... 41

Table 6-1: $\quad$ A Sample Processor Textual and Graphical Representation ............... 43

Table 6-2: $\quad$ Summary of Permitted Processor Declarations ............................. 44

Table 6-3: $\quad$ A Sample Memory Textual and Graphical Representation .................. 45

Table 6-4: $\quad$ Summary of Permitted Memory Declaration Subclauses ................... 46

Table 6-5: A Sample Bus Specification:

Textual and Graphical Representation....................................... 47

Table 6-6: $\quad$ Summary of Permitted Bus Declaration Subclauses .......................... 48

Table 6-7: A Sample Device Specification:

Textual and Graphical Representation......................................... 49

Table 6-8: $\quad$ Summary of Permitted Device Declaration Subclauses .................... 51

Table 7-1: A Sample System Specification:

Textual and Graphical Representation........................................ 53

Table 7-2: $\quad$ Summary of Permitted System Declarations .................................... 54

Table 8-1: $\quad$ Sample Declarations of Data, Event, and Event Data Ports................. 58

Table 8-2: $\quad$ AADL Specification of an Immediate Connection ............................... 63

Table 8-3: $\quad$ AADL Specification of a Delayed Connection .................................... 64

Table 8-4: $\quad$ Sample Port Group with Mixed Port Types ...................................... 68

Table 8-5: $\quad$ A Port Group Type Declaration and its Inverse ................................ 69

Table 8-6: $\quad$ Sample Port Group Connection Declarations ................................. 70

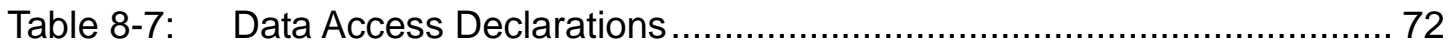

Table 8-8: $\quad$ Shared Access across a System Hierarchy .................................... 73

Table 8-9: $\quad$ Basic Bus Access and Access Connection Declarations ..................... 75 
Table 8-10: Example Bus Access Connection Declarations 76

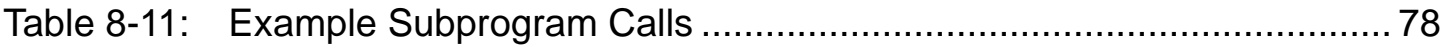

Table 8-12: Client-Server Subprogram Example ........................................ 79

Table 8-13: Example Parameter Connections ….......................................... 82

Table 8-14: Examples of Passing by Reference and Global Data ........................ 83

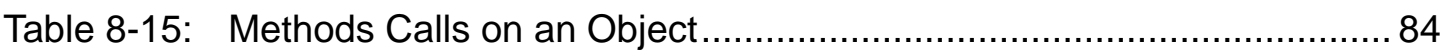

Table 9-1: $\quad$ Sample Graphical and Textual Specifications for Modes .................... 87

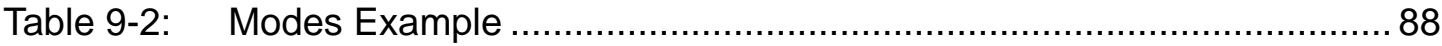

Table 9-3: $\quad$ Mode-Dependent Call Sequences ............................................... 89

Table 9-4: $\quad$ Mode-Specific Component Property Associations ............................. 90

Table 10-1: Flow Declarations within a Component Type Declaration................... 91

Table 10-2: Flow Implementation Declarations through a Component .................. 93

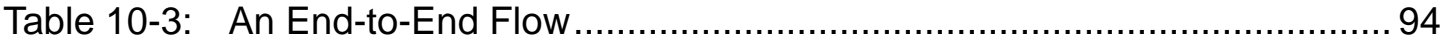

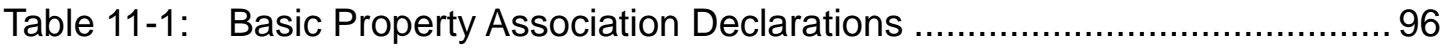

Table 11-2: Sample Access Property Associations ........................................ 97

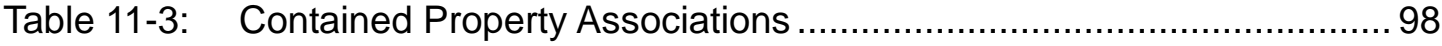

Table 11-4: In Binding and In Mode Property Associations ............................ 101

Table 11-5: Classifier Property Types …...................................................... 102

Table 11-6: Property Associations with Value ............................................... 103

Table 11-7: $\quad$ Sample Property Set Declarations ............................................ 104

Table 11-8: Sample Property Type Declarations .............................................. 105

Table 11-9: $\quad$ Sample Property Name Declarations............................................ 106

Table 11-10: Sample Property Constant Declarations .................................. 107 
Table 12-1: Example Package Declaration.................................................. 109

Table 12-2: Example Design Organization Using Packages ..............................110

Table 12-3: Example Type Extension ....................................................... 112

Table 12-4: Example Refines Type Implementation Subclauses........................113

Table 12-5: Example Implementation Extensions ..........................................114

Table 13-1: Allowed Component-Subcomponent Relationships........................117

Table 13-2: Allowed Features for Components ................................................118

Table 13-3: Features and Allowed Components .............................................119

Table 13-4: Constraints/Restrictions for Components .................................. 120

Table 13-5: AADL Built-in Property Types ................................................... 122

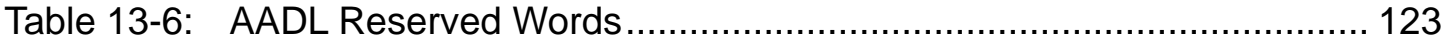

Table 13-7: Type Extensions and Associated Refinements .............................. 124

Table 13-8: Implementations Extensions and Associated Refinements .............. 125 


\section{Abstract}

In November 2004, the Society of Automotive Engineers (SAE) released the aerospace standard AS5506, named the Architecture Analysis \& Design Language (AADL). The AADL is a modeling language that supports early and repeated analyses of a system's architecture with respect to performance-critical properties through an extendable notation, a tool framework, and precisely defined semantics.

The language employs formal modeling concepts for the description and analysis of application system architectures in terms of distinct components and their interactions. It includes abstractions of software, computational hardware, and system components for ( $a$ ) specifying and analyzing real-time embedded and high dependability systems, complex systems of systems, and specialized performance capability systems and (b) mapping of software onto computational hardware elements.

The AADL is especially effective for model-based analysis and specification of complex realtime embedded systems. This technical note is an introduction to the concepts, language structure, and application of the AADL. 


\section{Introduction}

This document, Part 1 of a use guide for the Architecture Analysis \& Design Language (AADL), provides an introduction to the language and AADL specifications. ${ }^{1}$ The AADL is defined in the Society of Automotive Engineers (SAE) standard AS5506. ${ }^{2}$

\subsection{Document Summary}

Readers who are unfamiliar with the AADL will be able to gain a fuller understanding of the purpose, capabilities, notation, and elements of this modeling language. Table 1-1 summarizes the content in this document.

Table 1-1: Summary of Content in this Document

\begin{tabular}{|c|l|}
\hline $\begin{array}{c}\text { Section } \\
\text { Number }\end{array}$ & \multicolumn{1}{c|}{ Content Summary } \\
\hline 2 & $\begin{array}{l}\text { Section } 2 \text { summarizes the AADL language and introduces the AADL as a } \\
\text { framework for the design and analysis of the architectures of component-based } \\
\text { systems. }\end{array}$ \\
\hline 3 & $\begin{array}{l}\text { Section } 3 \text { provides a foundation for more detailed and problem-oriented } \\
\text { material in other sections of the document. This section also presents a } \\
\text { conceptual overview of the AADL abstractions; subsequent sections supply } \\
\text { details on the syntax and semantics of various language constructs. }\end{array}$ \\
\hline 4 & $\begin{array}{l}\text { Section } 4 \text { focuses on an AADL textual (natural language) specification as a } \\
\text { human-readable set of representations that consists of a collection of textual } \\
\text { declarations that comply with the AADL standard [SAE 06a]. The graphical } \\
\text { representations associated with the textual declarations are also included } \\
\text { throughout this document to highlight the relationship between the } \\
\text { representations. }\end{array}$ \\
\hline 5 & $\begin{array}{l}\text { Section } 5 \text { presents the software component abstractions (process, thread, } \\
\text { thread group data, and subprogram) and provides example declarations for } \\
\text { these components. }\end{array}$ \\
\hline 6 & $\begin{array}{l}\text { Section } 6 \text { provides the execution platform component abstractions (processor, } \\
\text { memory, bus, and device) and provides example declarations for these } \\
\text { components. }\end{array}$ \\
\hline 7 & $\begin{array}{l}\text { Section } 7 \text { discusses the system abstraction and presents examples of the } \\
\text { specification of composite systems and their instances. }\end{array}$ \\
\hline
\end{tabular}

1 The use guide for the AADL will be published initially as a series of technical notes.

2 For more information on the development, ongoing applications, and future plans of the AADL, go to http://www.aadl.info. To purchase a copy of the standard, go to http://www.sae.org/servlets /productDetail?PROD_TYP=STD\&PROD_CD=AS5506. 
Section 1: Introduction

Table 1: Summary of Content in this Document (cont.)

\begin{tabular}{|c|l|}
\hline $\begin{array}{c}\text { Section } \\
\text { Number }\end{array}$ & \multicolumn{1}{c|}{ Content Summary } \\
\hline 8 & $\begin{array}{l}\text { Section 8 describes the abstractions that support the specification of } \\
\text { component interactions. Examples of the specification of component interfaces } \\
\text { and their interconnections are presented. }\end{array}$ \\
\hline 9 & $\begin{array}{l}\text { Section 9 presents the specification of alternative operational states of a } \\
\text { system. Modes mode transitions, and examples of their specification are } \\
\text { described. }\end{array}$ \\
\hline 10 & $\begin{array}{l}\text { Section 10 describes the use of the AADL flows concept and presents } \\
\text { examples of the specification of abstract flows throughout a system. }\end{array}$ \\
\hline 11 & $\begin{array}{l}\text { Section 11 discusses property constructs and presents examples of property } \\
\text { type and name definitions, property set declarations, and property associations. }\end{array}$ \\
\hline 12 & $\begin{array}{l}\text { Section 12 describes the constructs for organizing an AADL specification. It } \\
\text { includes examples of AADL architectural pattern sets. }\end{array}$ \\
\hline
\end{tabular}

The Appendix (pages 117-125) provides tabular summaries of the features, components, and built-in properties of the language.

\subsection{Reader's Guide to Technical Interests}

Readers familiar with the AADL standard document will be able to take advantage of the detailed descriptions and examples (in textual and graphical forms) shown in the technical interest areas that are correlated with sections in this document in Table 1-2.

Table 1-2: Technical Interests and Relevant Sections in this Document

\begin{tabular}{|l|l|}
\hline \multicolumn{1}{|c|}{ Section Numbers } & \multicolumn{1}{c|}{ Technical Considerations } \\
\hline $\begin{array}{l}5.4,5.5,8.3 .1,8.3 .2,8.4, \\
\text { and } 8.5\end{array}$ & $\begin{array}{l}\text { Modeling Application Software-These sections address data } \\
\text { and subprogram components and their interactions (e.g., calls } \\
\text { and component access. }\end{array}$ \\
\hline $\begin{array}{l}5.1,5.2,5.3,8.1,8.2, \\
8.3 .1,8.3 .2, \text { and 8.4.2 }\end{array}$ & $\begin{array}{l}\text { Execution Tasking and Concurrency-These sections present } \\
\text { relevant aspects of runtime interaction, coordination, and timing } \\
\text { associated with multiple execution paths. }\end{array}$ \\
\hline 6,7, and 8.3.3 & $\begin{array}{l}\text { System Instances and Binding Software to Hardware } \\
\text { Components-These sections discuss issues and capabilities in } \\
\text { defining a complete instance of a system architecture. }\end{array}$ \\
\hline 11 & $\begin{array}{l}\text { Properties of Model Elements-This section discusses assigning } \\
\text { values to properties and defining new properties within an AADL } \\
\text { model. }\end{array}$ \\
\hline
\end{tabular}


Table 1-2: $\quad$ Technical Interests and Relevant Sections in this Document (cont.)

\begin{tabular}{|l|l|}
\hline \multicolumn{1}{|c|}{ Section Numbers } & \multicolumn{1}{c|}{ Technical Considerations } \\
\hline 9 and 11.2 & $\begin{array}{l}\text { Partitioning Runtime Configurations-These sections present the } \\
\text { structuring of alternative architectural configurations for a system. }\end{array}$ \\
\hline $10,11.3,11.4$, and 11.5 & $\begin{array}{l}\text { Analysis Abstractions-These sections discuss capabilities that } \\
\text { facilitate analysis of a system architecture. }\end{array}$ \\
\hline
\end{tabular}

\subsection{Conventions Used in this Document}

The textual and graphical illustrations used in this technical note reflect the styles used in the AADL standard document [SAE 06a], except where noted. In addition, for consistency and clarification in this document, we have represented AADL core language concepts and key specification elements the same way (i.e., using the same type style and format) in textual examples and explanatory text (in sections 4 through 12). Also, we have used the AADL icon (A) to indicate a different semantics than that represented by a similar graphical symbol in the Unified Modeling Language (UML). 


\section{SAE AADL Overview}

The SAE AADL standard provides formal modeling concepts for the description and analysis of application systems architecture in terms of distinct components and their interactions. The AADL includes software, hardware, and system component abstractions to

- $\quad$ specify and analyze real-time embedded systems, complex systems of systems, and specialized performance capability systems

- map software onto computational hardware elements

The AADL is especially effective for model-based analysis and specification of complex realtime embedded systems.

\subsection{Abstraction of Components}

Within the AADL, a component is characterized by its identity (a unique name and runtime essence), possible interfaces with other components, distinguishing properties (critical characteristics of a component within its architectural context), and subcomponents and their interactions.

In addition to interfaces and internal structural elements, other abstractions can be defined for a component and system architecture. For example, abstract flows of information or control can be identified, associated with specific components and interconnections, and analyzed. These additional elements can be included through core AADL language capabilities (e.g. defining new component properties) or the specification of a supplemental annex language. ${ }^{3}$

The component abstractions of the AADL are separated into three categories:

1. application software

a. thread: active component that can execute concurrently and be organized into thread groups

b. thread group: component abstraction for logically organizing thread, data, and thread group components within a process

c. process: protected address space whose boundaries are enforced at runtime

d. data: data types and static data in source text

e. subprogram: concepts such as call-return and calls-on methods (modeled using a subprogram component that represents a callable piece of source code)

3 Annex libraries enable a designer to extend the language and customize an AADL specification to meet project- or domain-specific requirements. An annex document is an approved extension to the core AADL standard. [SAE 06a]. 
2. execution platform (hardware)

a. processor: schedules and executes threads

b. memory: stores code and data

c. device: represents sensors, actuators, or other components that interface with the external environment

d. bus: interconnects processors, memory, and devices

3. composite

a. system: design elements that enable the integration of other components into distinct units within the architecture

System components are composites that can consist of other systems as well as of software or hardware components.

The AADL standard includes runtime semantics for mechanisms of exchange and control of data, including

- message passing

- $\quad$ event passing

- synchronized access to shared components

- thread scheduling protocols

- timing requirements

- remote procedure calls

In addition, dynamic reconfiguration of runtime architectures can be specified using operational modes and mode transitions.

\subsection{Architectural Analysis}

The AADL can be used to model and analyze systems already in use and design and integrate new systems. The AADL can be used in the analysis of partially defined architectural patterns (with limited architectural detail) as well as in full-scale analysis of a complete system model extracted from the source code (with completely quantified system property values).

AADL supports the early prediction and analysis of critical system qualities - such as performance, schedulability, and reliability. For example, in specifying and analyzing schedulability, AADL-supported thread components include the predeclared ${ }^{4}$ execution property options of periodic, aperiodic (event-driven), background (dispatched once and executed to completion), and sporadic (paced by an upper rate bound) events. These thread characteristics are defined as part of the thread declaration and can be readily analyzed.

4 There is a standard predeclared property set named AADL_Properties that is part of every AADL specification [SAE 06a]. 
Within the core language, property sets can be declared that include new properties for components and other modeling elements (e.g. ports and connections). By utilizing the extension capabilities of the language, too, additional models and properties can be included. For example, a reliability annex can be used that defines reliability models and properties of components facilitating a Markov or fault tree analysis of the architecture [SAE 06b]. This analysis would assess an architecture's compliance with specific reliability requirements.

Collectively, these AADL properties and extensions can be used to incorporate new and focused analyses at the architectural design level. These analyses facilitate tradeoff assessments among alternative design options early in a development or upgrade process.

AADL components interact exclusively through defined interfaces. A component interface consists of directional flow through

- data ports for unqueued state data

- event data ports for queued message data

- event ports for asynchronous events

- synchronous subprogram calls

- explicit access to data components

Interactions among components are specified explicitly. For example, data communication among components is specified through connection declarations. These can be midframe (immediate) communication or phase-delayed (delayed) communication. The semantics of these connections assures deterministic transfer of data streams. Deterministic transfer means that a thread always receives data with the same time delay; if the receiving thread is over- or under-sampling the data stream, it always does so at a constant rate.

Application components have properties that specify timing requirements such as period, worst-case execution time, deadlines, space requirements, arrival rates, and characteristics of data and event streams. In addition, properties identify the following:

- $\quad$ source code and data that implement the application component being modeled in the AADL

- constraints for binding threads to processors, source code, and data onto memory

The constraints can limit binding to specific processor or memory types (e.g., to a processor with DSP support) as well as prevent colocation of application components to support fault tolerance [Feiler 04]. 


\section{AADL Language Abstractions}

The core language concepts and key specification elements of AADL are summarized in Figure 3-1. In AADL, components are defined through type and implementation declarations. A Component Type declaration defines a component's interface elements and externally observable attributes (i.e., features that are interaction points with other components, flow specifications, and internal property values). A Component Implementation declaration defines a component's internal structure in terms of subcomponents, subcomponent connections, subprogram call sequences, modes, flow implementations, and properties. Components are grouped into application software, execution platform, and composite categories. Packages enable the organization of AADL elements into named groups. Property Sets and Annex Libraries enable a designer to extend the language and customize an AADL specification to meet project- or domainspecific requirements. ${ }^{5}$

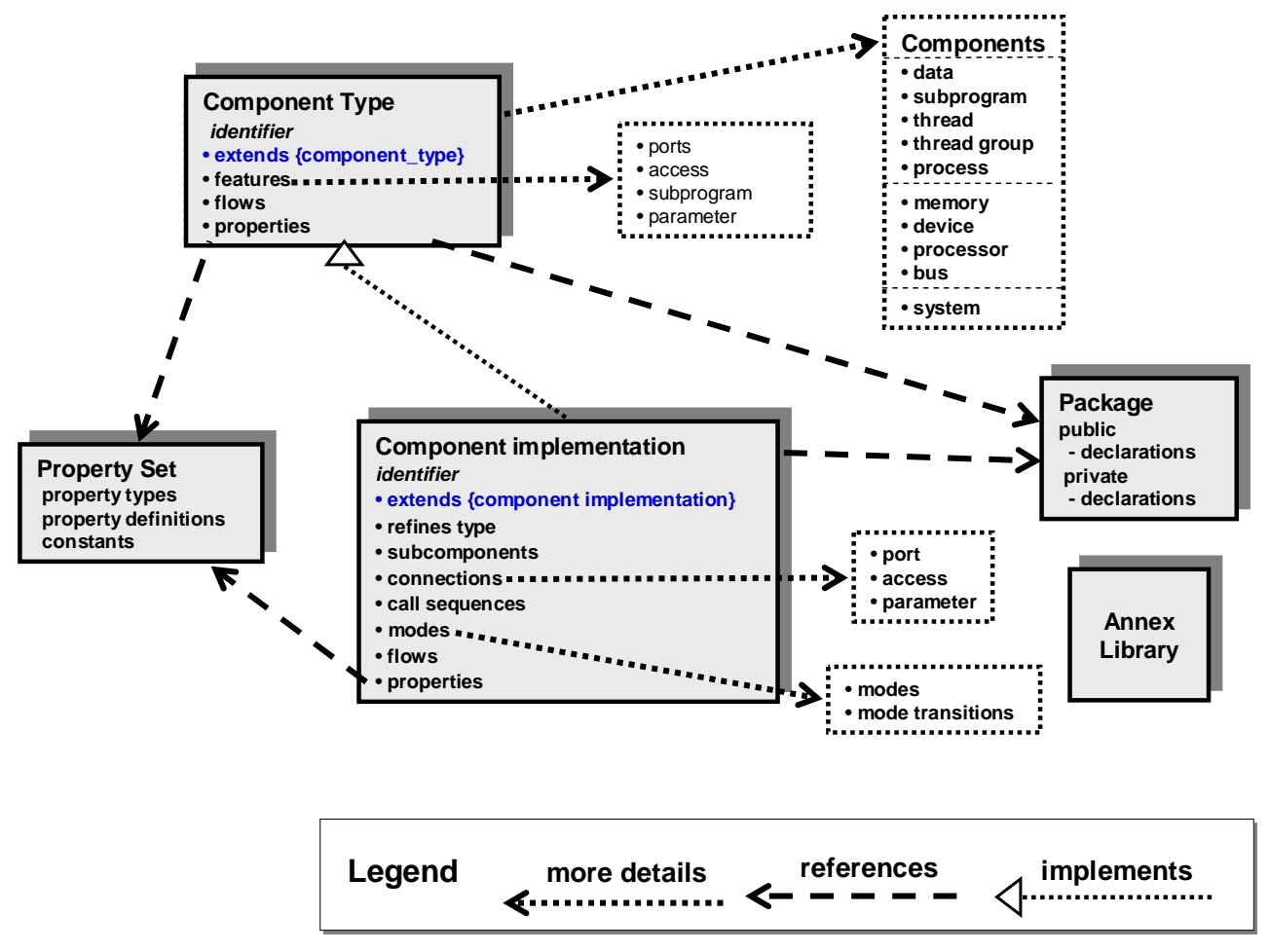

Figure 3-1: $\quad$ Summary of AADL Elements

5 Annex libraries enable a designer to extend the language and customize an AADL specification to meet project- or domain-specific requirements. An annex document is an approved extension to the core AADL standard. 


\subsection{Components}

Components form the central modeling vocabulary for the AADL. Components are assigned a unique identity (name) and are declared as a type and implementation within a particular component category. A component category defines the runtime essence of a component. There are three distinct sets of component categories:

1. application software

a. thread: a schedulable unit of concurrent execution

b. thread group: a compositional unit for organizing threads

c. process: a protected address space

d. data: data types and static data in source text

e. subprogram: callable sequentially executable code

2. execution platform
a. processor: components that execute threads
b. memory: components that store data and code
c. device: components that interface with and represent the external environment
d. bus: components that provide access among execution platform components

3. composite

a. system: a composite of software, execution platform, or system components

Each of the component categories is discussed in separate sections of this document. The syntax and semantics of declarations in an AADL specification are discussed in Section 4.1.

\subsection{Component Types}

An AADL component type declaration establishes a component's externally visible characteristics. For example, a declaration specifies the interfaces of a thread component. A component type declaration consists of a defining clause and descriptive subclauses; Figure 3-2 shows a type declaration of a thread. Features are the interfaces of the component. Flows specify distinct abstract channels of information transfer. Properties define intrinsic characteristics of a component. There are predefined properties for each component category (e.g., the execution time for a thread).

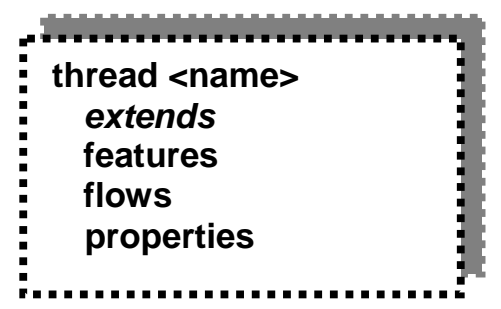

Figure 3-2: $\quad$ Subclauses of a Type Declaration 
The extends subclause enables one component type declaration to build upon another. A component declared as an extension inherits the characteristics of the original component (i.e., it is a subclass of the original). Within a component declared as an extension of another type, interfaces, flows, and properties can be added; partially declared elements of the antecedent component type can be detailed; and properties can be modified (refined). These qualities permit the modeling of variations in the interfaces of a family of related components.

\subsection{Component Implementations}

A component implementation specifies an internal structure in terms of subcomponents, interactions (calls and connections) among the features of those subcomponents, flows across a sequence of subcomponents, modes that represent operational states, and properties.

The subclauses of an implementation declaration are summarized in Figure 3-3. The subcomponents, connections, and calls declarations specify the composition of a component as a collection of components (subcomponents) and their interactions. Flows represent implementations of flow specifications in the component type or end-to-end flows to be analyzed (i.e., flows that start in one subcomponent, go through zero or more subcomponents, and end in another subcomponent). Modes represent alternative operational modes that may manifest themselves as alternate configurations of subcomponents, calls sequences, connections, flow sequences, and properties. Properties define intrinsic characteristics of a component. There are predefined properties for each component implementation.

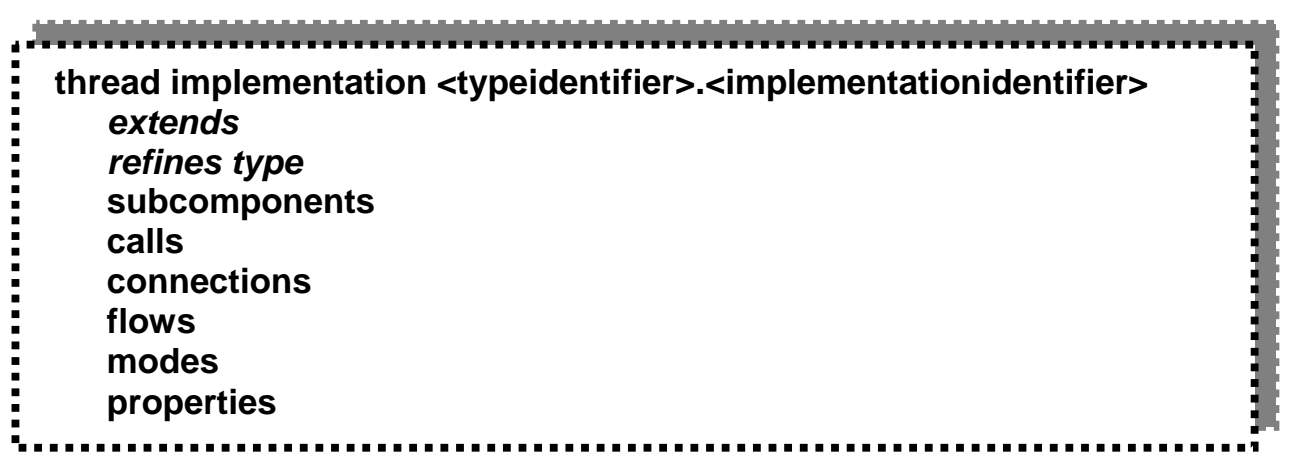

Figure 3-3: $\quad$ Subclauses of an Implementation Declaration

Multiple implementations of a component type can be declared, allowing multiple variants with the same external interfaces to be modeled because each implementation provides a realization of a component that satisfies the same interface specified by the component type. In addition, a component implementation may extend and refine other previously declared component implementations. Extended implementations (declared with the extends subclause) inherit the characteristics of the original component implementation and all of its predecessors. Refinement allows partially specified 
component implementations (templates) to be completed, while extension allows a component implementation to be expressed as variation of a common component description through additions. In addition, an extends implementation declaration can add property values to the features of its corresponding type. These additions can be made through the refines type subclause.

Component decomposition is defined through subcomponents declarations within component implementation declarations. A subcomponent represents the decomposition element and the classifier (named implementation) represents a choice in a family. A component instance is created by instantiating a component implementation and each of its subcomponents recursively.

\subsection{Packages, Property Sets, and Annexes}

AADL packages permit collections of component declarations to be organized into separate units with their own namespaces. Elements with common characteristics (e.g., all components associated with network communications) can be grouped together in a package and referenced using the package name. Packages can support the independent development of AADL models for different subsystems of a large-scale system by providing a distinct namespace for each group of subsystem elements.

A property set is a named grouping of property declarations that define new properties and property types that can be included in a specification. For example, a security property set can include definitions for security levels required in a database system. These properties are referenced using the property set name and can be associated with components and other modeling elements (e.g., ports or connections) within a system specification. Their declaration and use become part of the specification.

An annex enables a user to extend the AADL language, allowing the incorporation of specialized notations within a standard AADL model. For example, a formal language that enables an analysis of a critical aspect of a system (e.g., reliability analysis, security, or behavior) can be included within an AADL specification. ${ }^{6}$

Each of these elements is described in more detail in other sections of this document.

6 Annex libraries enable a designer to extend the language and customize an AADL specification to meet project- or domain-specific requirements. An annex document is an approved extension to the core AADL standard. 


\section{AADL System Models and Specifications}

An AADL system model describes the architecture and runtime environment of an application system in terms of its constituent software and execution platform (hardware) components and their interactions. An AADL model is captured in a specification consisting of syntactically and semantically correct AADL declarations. A complete AADL system model includes all of the declarations required to instantiate a runtime instance of an application system that the specification represents (e.g., an aircraft's flight control system).

From a user perspective, an AADL specification and its constituent declarations can be expressed textually, graphically, in a combination of those representations, or as Extensible Markup Language (XML). The AADL textual and graphical notations are defined by the SAE AADL standard and its extensions [SAE 06a]. The XML form is defined in Extensible Markup Language (XML) 1.0 (Third Edition) [W3C 04]. Figure 4-1 summarizes the alternative representations of an AADL specification, showing sample textual, graphical, and XML representations.

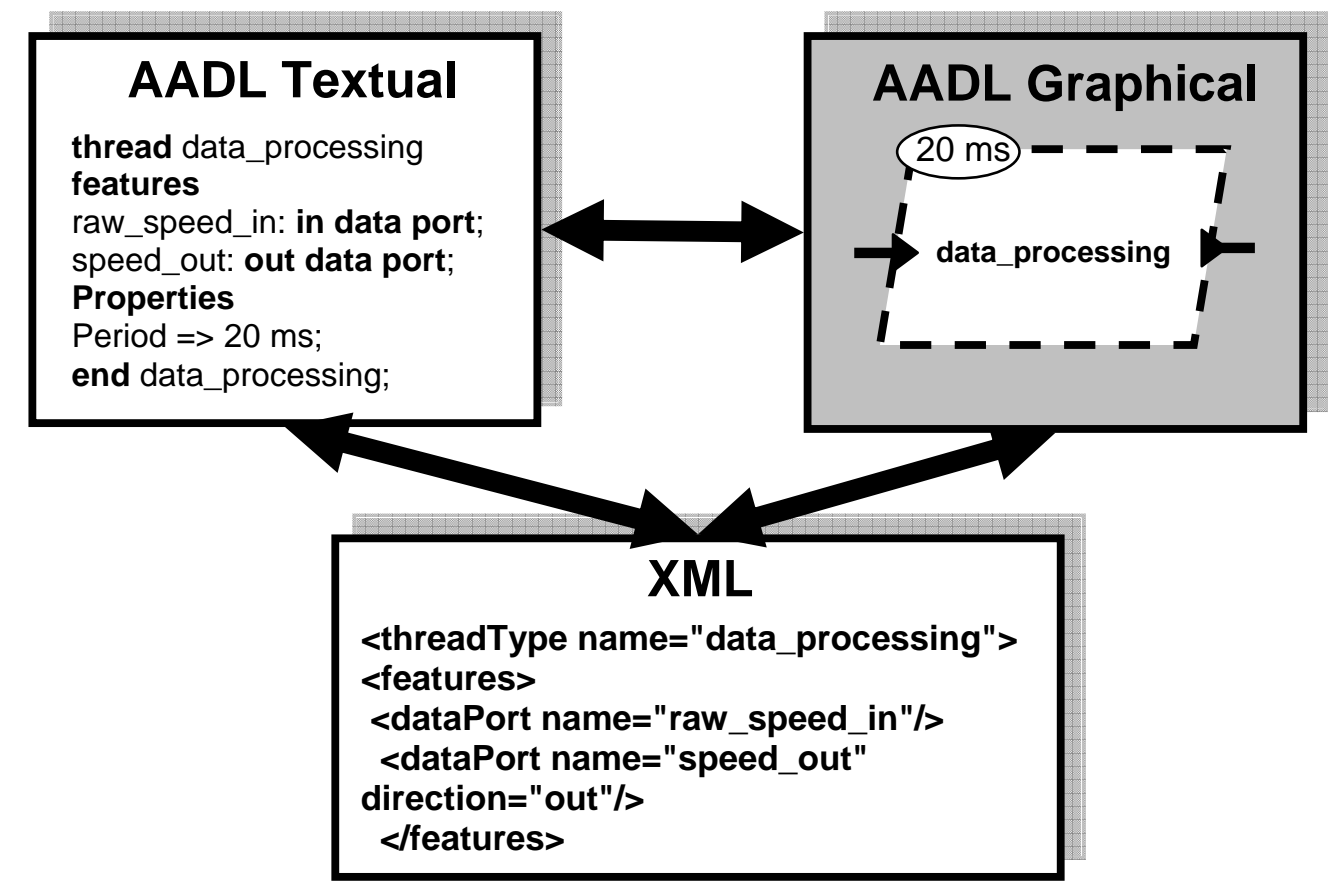

Figure 4-1: $\quad$ AADL Representations 


\subsection{AADL Textual Specifications}

This section focuses on an AADL textual (natural language) specification as a humanreadable collection of textual declarations that comply with the AADL standard [SAE 06a]. Graphical notations associated with the textual specifications are included in this document to highlight the relationship between representations and to help the reader visualize the architecture. Detailed descriptions of the graphical representations for AADL constructs and declarations are provided in the graphical standard. ${ }^{7}$ The principal AADL declarations are summarized in Table 4-1.

Table 4-1: Principal AADL Declarations

\begin{tabular}{|c|c|}
\hline Declaration & Description \\
\hline $\begin{array}{l}\text { Component Type: } \\
\text { system, process, thread, thread } \\
\text { group data, subprogram, } \\
\text { processor, device, memory, and } \\
\text { bus }\end{array}$ & $\begin{array}{l}\text { The component type declaration establishes the } \\
\text { identity (component category and name) and } \\
\text { defines the features, flows, and properties of a } \\
\text { component type. A component type declaration may } \\
\text { also declare the type as an extension of another } \\
\text { type (extends). }\end{array}$ \\
\hline $\begin{array}{l}\text { Component Implementation: } \\
\text { system, process, thread, thread } \\
\text { group data, subprogram, } \\
\text { processor, device, memory, and } \\
\text { bus }\end{array}$ & $\begin{array}{l}\text { The component implementation declaration } \\
\text { establishes the identity (component category, type, } \\
\text { and name) and defines the refinements (refines } \\
\text { type subclause), subcomponents, calls, } \\
\text { connections, flows, modes, and properties of a } \\
\text { component implementation. The identity must } \\
\text { include a declared component type consistent with } \\
\text { the component category. The component } \\
\text { implementation declaration may also declare the } \\
\text { implementation as an extension of another } \\
\text { implementation (extends subclause). }\end{array}$ \\
\hline Port Group Type & $\begin{array}{l}\text { Port group type declarations establish the identity } \\
\text { (name) and define the features and properties of a } \\
\text { grouping of ports and/or port groups. Within the } \\
\text { features declaration, a port group may be defined } \\
\text { as the inverse of another port group. A port group } \\
\text { type declaration may also declare the port group as } \\
\text { an extension of another port group type (extends). }\end{array}$ \\
\hline Package & $\begin{array}{l}\text { The package declaration establishes the identity } \\
\text { (name) of a collection of AADL declarations, groups } \\
\text { those declarations into private and public sections, } \\
\text { and declares properties associated with a package. } \\
\text { Packages are used to logically organize AADL } \\
\text { declarations. AADL component type, } \\
\text { implementation, and port group declarations placed } \\
\text { in AADL packages can be referenced by } \\
\text { declarations in other packages. }\end{array}$ \\
\hline
\end{tabular}

7 The complete set of graphical symbols for AADL components is presented in "Graphical AADL Notation," a draft document at the time of the publishing of this technical note. 
Table 4-1: AADL Declarations (cont.)

\begin{tabular}{|c|c|}
\hline Property Set & $\begin{array}{l}\text { Property set declarations introduce additional } \\
\text { properties, property types, and property constants } \\
\text { that are not included as predeclared AADL } \\
\text { properties. }{ }^{8} \text { Each property set has a unique global } \\
\text { name and provides a unique namespace for the } \\
\text { items declared in it. In other words, properties and } \\
\text { property types declared in a property set are } \\
\text { referenced by property set name and item name. }\end{array}$ \\
\hline Annex Library & $\begin{array}{l}\text { Annex library declarations establish the identity } \\
\text { (name) and define the contents of a set of reusable } \\
\text { declarations that are not part of the standard AADL } \\
\text { language. Annex declarations are used to extend } \\
\text { AADL's core modeling and analysis capabilities. }\end{array}$ \\
\hline
\end{tabular}

\subsection{Graphical Representations}

The AADL's graphical notation facilitates a clear visual presentation of a system's structural hierarchy and communication topology and provides a foundation for distinct architecture perspectives. Graphical notation elements for AADL components are shown in Figure 4-2. The letter-shaped AADL icon (A) is used to indicate a different semantics than that represented by a similar graphical symbol in the Unified Modeling Language (UML). This symbol is not required in notation; it can be used where a distinction from a UML symbol is necessary. Additional symbols, such as circles, are used to represent component properties (e.g., the period of a thread).
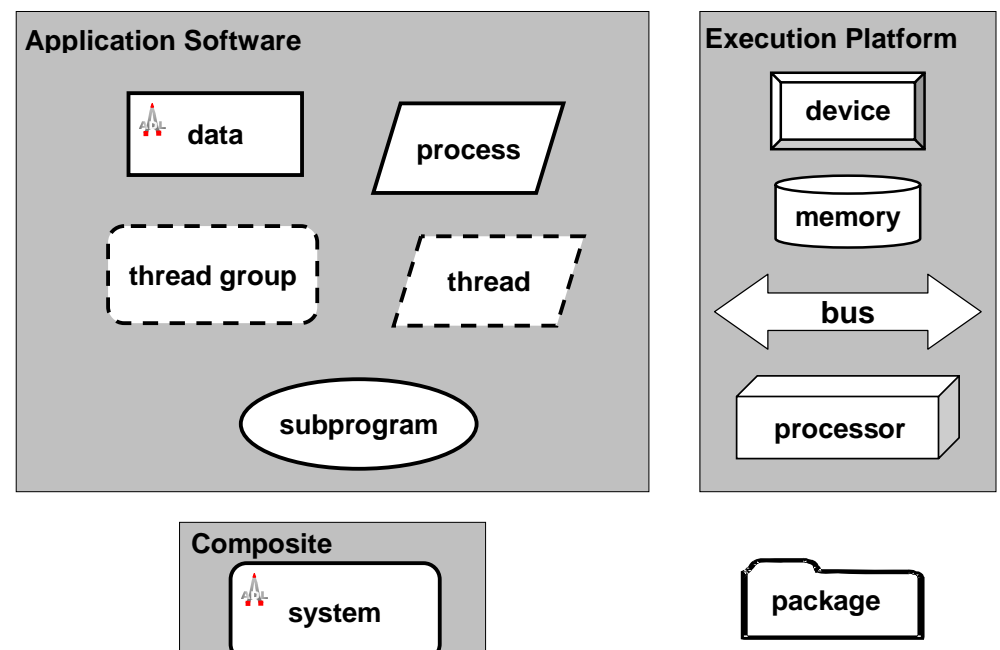

Figure 4-2: $\quad$ AADL Graphical Notation

8 There is a standard predeclared property set named AADL_Properties that is a part of every AADL specification [SAE 06a]. 


\subsection{Example Specification}

Table 4-2 contains an excerpt from an AADL textual specification and includes sample graphical representations of portions of the specification. ${ }^{9}$ The excerpt shows simplified component type, component implementation, and subcomponents declarations (i.e., only some of the features, flows, or properties are declared) and illustrates the pattern other examples in this document will follow.

In the example shown in Table 4-2, related type and implementation declarations are grouped together. Individual declarations can be arranged in any order within an AADL specification. For example, a component type declaration that includes a specific port group as one of its interfaces (features) can precede that port group's type declaration. An alternative organization might involve grouping together all type declarations. In addition, all or some of the declarations shown in Table 4-2 can be partitioned into groups using packages. The options provided by packages and their implications are discussed in Section 12 (Organizing a Specification).

The excerpt in Table 4-2 contains one process and two thread component type declarations. The process type definition has the component type identifier (name) control_processing. Two data ports, in data port and out data port, are declared for this process type. The sensor_data and command_data data types are declared in individual data type declarations.

The thread type definition identifiers are control_in and control_out. An implementation declaration of the process type control_processing is shown. The component implementation identifier is speed_control. An

implementation is referenced by using both the component type identifier and the component implementation identifier, separated by a period (.). A reference to a thread implementation input_processing_01 of the thread type control_in is shown in the declaration of the subcomponent control_input. Thus, control_input is an instance of the component implementation control_in.input_processing_01.

Graphical representations of the process type declaration control_processing and the process implementation declaration are shown in the latter portions of Table 4-2. The process implementation symbol in the example is bounded with a bold line. Bold-lining of an implementation symbol is optional. It can be useful in distinguishing component type and component implementation representations visually. A solid black triangular symbol represents a data port. Port and other features symbols are discussed in Section 8 (Component Interactions).

9 In the example specifications shown here and in Sections 5-12, we typically follow the pattern of displaying the textual representation followed by the graphical representation in portions of the same table, as shown in Table 4-2. Where needed to provide clarification, we have placed the textual and graphical representations in separate tables and figures. 
Section 4: AADL System Models and Specifications

Table 4-2: A Simplified Example of an AADL Specification ${ }^{10}$

- - A process type definition with the component type

- - identifier (name) "control_processing" is shown below.

process control_processing

features

input: in data port sensor_data;

output: out data port command_data;

end control_processing;

- - Below is an implementation of process type "control_processing"

- - The component implementation identifier(name)is "speed_control"

- - The implementation is referenced by using both the component type

- - identifier and the component implementation identifier, separated

- - by a period(.)in the form: control_processing.speed_control.

- - A reference to a thread implementation "input_processing_01"

- - of the thread type "control_in" is shown below in the

- - declaration of the subcomponent "control_input"

process implementation control_processing.speed_control subcomponents

control_input: thread control_in.input_processing_01;

control_output: thread control_out.output_processing_01;

end control_processing.speed_control;

-- The declaration of the thread type "control_in" is shown below.

thread control_in

end control_in;

- - The declaration of the thread implementation

- - "control_in.input_processing_01" is shown below.

thread implementation control_in.input_processing_01

end control_in.input_processing_01;

-- The declaration of the thread type "control_out" is shown below.

thread control_out

end control_out;

- - The declaration of the thread implementation

- - "control_out.output_processing_01" is shown below.

thread implementation control_out.output_processing_01

end control_out.output_processing_01;

- - The declaration of the data type "sensor_data" is shown below.

data sensor_data

end sensor_data;

- - The declaration of the data type "command_data" is shown below. data command_data

end command_data;

${ }^{10}$ Comment lines in an AADL specification are prefaced by two dashes (--). 
Section 4: AADL System Models and Specifications

Table 4-2: A Simplified Example of an AADL Specification (cont.)

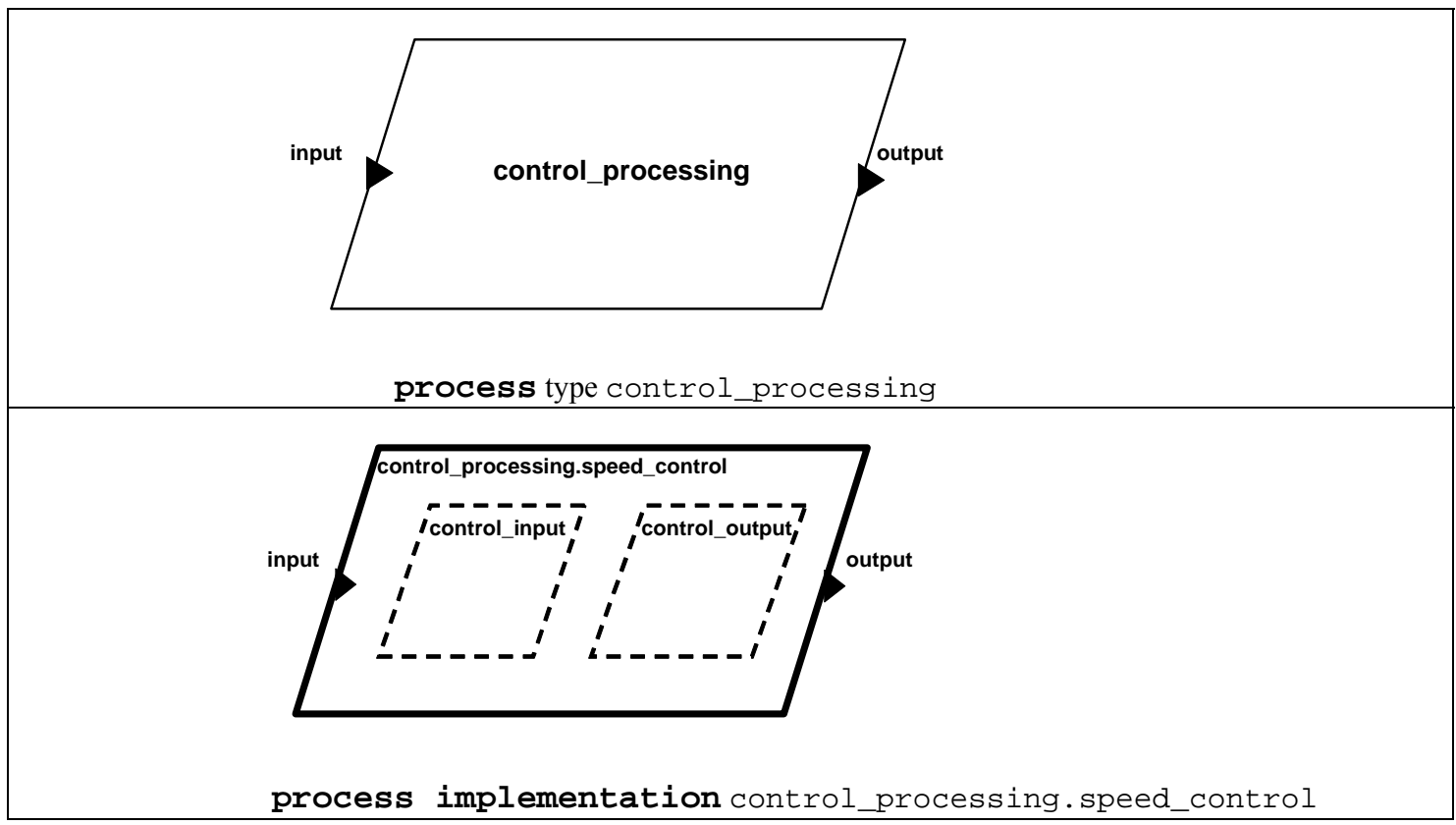

\subsection{Type Declarations}

The structures for a component type declaration (area labeled (1)) and a type declaration that extends another type (area labeled (2)) are shown in Table 4-3, along with sample component type declarations (area labeled (3). The sample type declarations are for a process type simple_speed_control and a thread type data_management. The first line of each declaration begins with the appropriate component category reserved word in boldface. In these examples, process and thread are reserved words.

Table 4-3: Sample Component Type Declarations

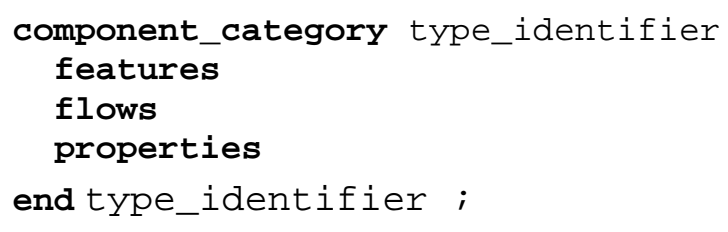


Table 4-3: $\quad$ Sample Component Type Declarations (cont.)

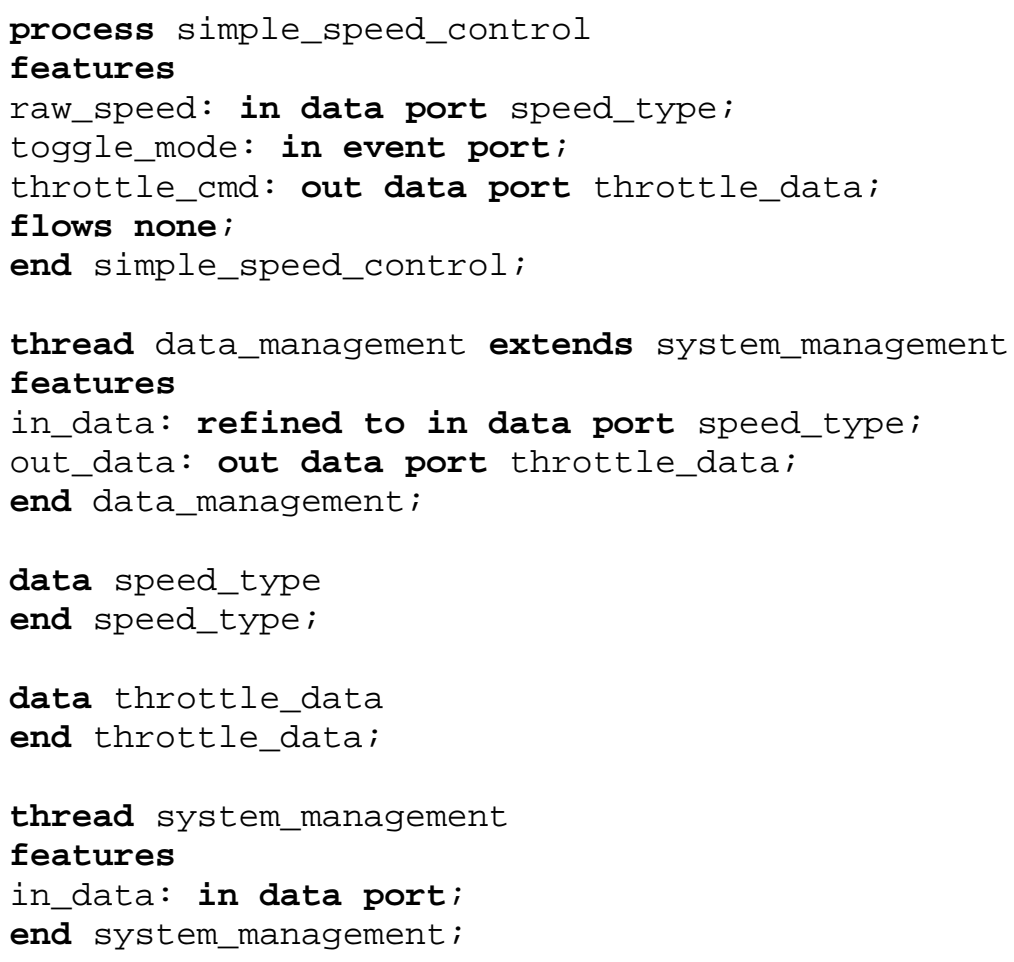

The component type classifier (name) of the type follows the component category reserved word. A component type declaration may contain up to four subclauses that are identified with these reserved words:

- features: specifies the interaction points with other components, including the inputs and accesses required by the component and all the outputs and items the component provides

- flows: defines specifications of logical flows through the component from incoming interaction points to outgoing interaction points (These flows can be used to specify endto-end flows without having to expose or have available any implementation detail of the component. Flows can trace data, control, or mixed flow by connecting event and data ports.)

- properties: specifies properties of the component that apply to all instances of this component unless overwritten in implementations or extensions

- extends: is used where a type extends another type, as shown for the thread type data_management in Table 4-3

If there are no entries under the subclause reserved words features, flows, or properties, they may be omitted, or the reserved word statement none can be used to signify explicitly that there are no entries. For example, the reserved word subclause flows is omitted in the thread type declaration for data_management and none is used in the 
other empty subclause cases in Table 4-3. The use of none explicitly designates that the subclause is empty. The use of none avoids the misinterpretation of a developer's accidental omission of a subclause declaration as intentional.

In Table 4-3, these declarations under the features subclause in the type declaration for simple_speed_control define ports for the type:

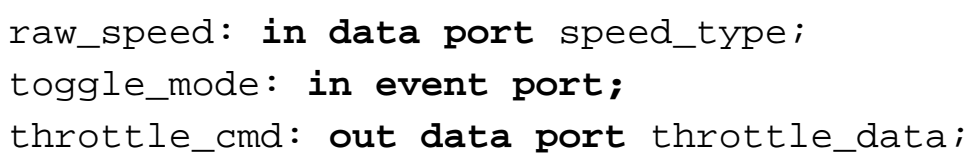

Notice that there is one in data port declaration in the features section of the type system_management. The type declaration for data_management extends the type system_management. Within this type extension declaration, the in data port in_data declaration is completed by including refined to and adding the data type speed_type to the port declaration, and an out data port declaration is added.

A component type declaration is terminated by the reserved word end followed by the component's type classifier and a semicolon (;).

\subsection{Implementation Declarations}

A component implementation declaration structure (1) and (2) and a sample declaration (3) are shown in Table 4-4. The basic form (1) declares a distinct implementation. The second form (2) includes the reserved word extends, indicating that the declared implementation extends another.

In the sample declaration (3) in Table 4-4), a thread implementation with the name control_laws.control_input is declared as an implementation of the type control_laws. The implementation name is formed using the type identifier followed by a specific identifier for the implementation. These are separated by a period (.). Within the control_laws.control_input declaration, a single data subcomponent is declared, the reserved word statement (none) is used for the calls subclause, and the other subclauses are omitted. 
Table 4-4: Component Implementation Declarations

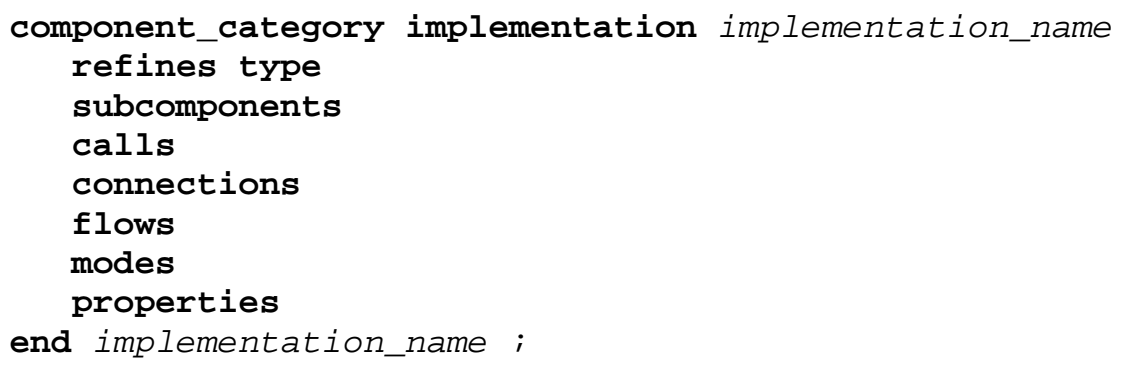

\subsection{Package Declarations}

Packages provide a way to organize component type declarations, implementation declarations, and property associations within an AADL specification. Each package introduces a distinct namespace for component classifier declarations, port group type declarations, annex library declarations, and property associations.

For example, a component type may be declared within a package and used in multiple subsystem declarations. This is shown in Table 4-5 where the package acutators_sensors includes a device speed_sensor that is used in the primary and backup implementation of the system control. Note that the package name with a double colon (: :) is used to precede the device speed_sensor when it is referenced (e.g., in the subcomponent declaration within the implementation declarations). The comment line ( - - ...) is used to indicate other declarations that are not shown. Packages are discussed in more detail in Section 12.1 (Packages). 
Table 4-5: Example Packages

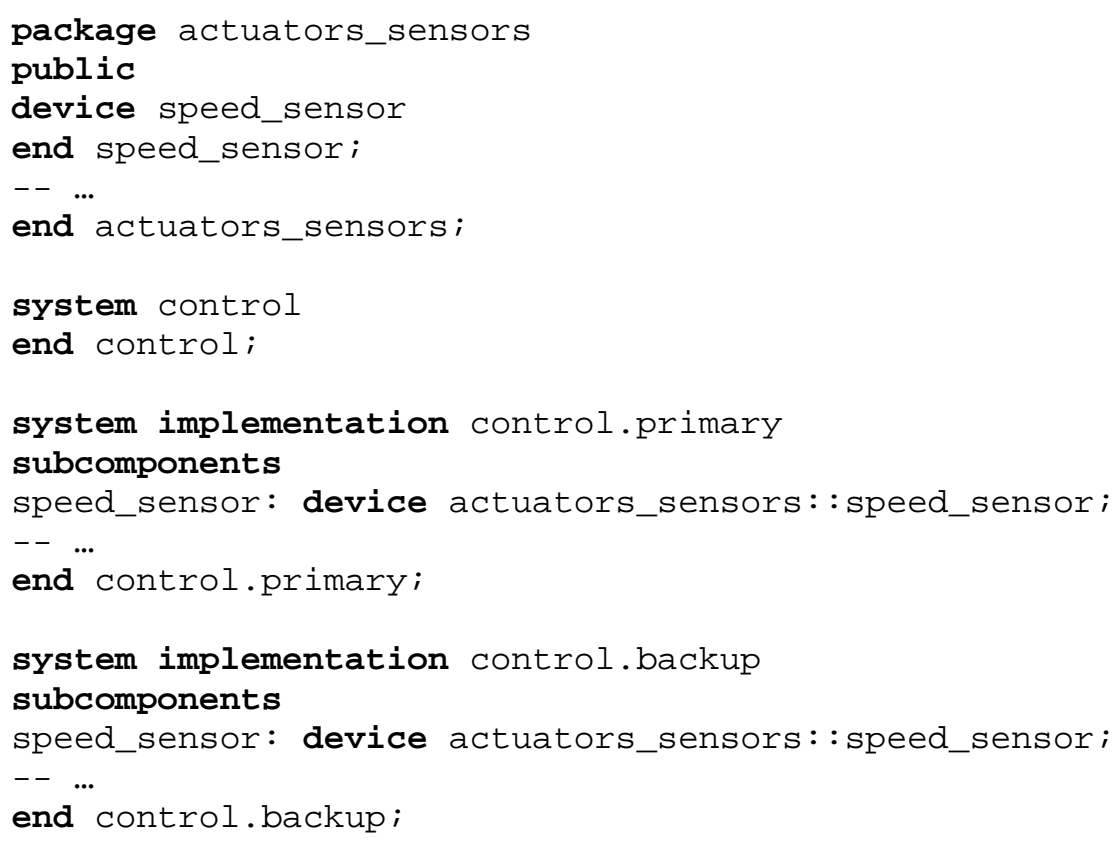

\subsection{Property Set Declarations}

Property set declarations allow the addition of properties to the core AADL property set. These additions can be used to support specialized modeling and analysis capabilities that can be defined in AADL annexes. Declarations in an AADL specification can refer to packages and property sets that may be separately stored. More details on property set declarations can be found in Section 11.3 (Defining New Properties). References to property names, types, and constants declared within a property set are prefaced by the name of the property set.

\subsection{Annex Library Declarations}

Annex library declarations enable extensions to the core language concepts and syntax. Often these extensions support custom analyses using specialized models and abstractions (e.g., an error annex that supports reliability analysis). Annex libraries define a sublanguage that can be used in annex subclauses within component type and implementation declarations. Annex libraries are declared within packages and annex subclauses can be included within component type and implementation declarations. These subclauses use the elements declared in the annex library (e.g., associating values or expressing assertions with elements of the annex). ${ }^{11}$

11 The language can also be extended through annex documents, which are approved extensions to the core AADL standard. 


\subsection{Namespaces}

There is a global namespace for an AADL specification. Packages and property set names are in the global namespace. Their content can be named anywhere by preceding it with the package name. Component declarations placed directly in an AADL specification are visible only within that AADL specification. They are not accessible from within packages or other AADL specifications; they are considered to reside in an anonymous namespace. An AADL specification acts as a local work area whose component declarations are only locally visible.

\subsection{Partial Specifications}

A number of declarations within a syntactically and semantically correct specification can be partially completed. For example, neither the identity (type or implementation) of a component contained within another nor the data type for the ports in a data connection between components needs to be specified until a complete representation is instantiated from the specification (i.e., the design is finalized).

The flexibility to develop partial specifications can be used effectively during design, especially in the early stages where details may not be known or decided upon. This flexibility allows the syntactic checking of an incomplete specification and enables extended semantic, domain, or project-specific analysis to be conducted. For example, the detailed signal timing can be specified and signal latency can be analyzed without a complete or detailed specification of the representation of data communicated through ports or other elements of the design. Similarly, using the flow specification construct, end-to-end flows can be analyzed without the system hierarchy being detailed to the level required for instantiation.

\subsection{Extends, Refines, and Partial Specification}

When coupled with the extends, refines, and implementation facilities of the language, partial specification can be used to define a core type or implementation pattern. This core pattern can be used to generate a family of components (i.e., core patterns with less detail and descendants with more specific and modified declarations). Table 4-6 shows an example of the use of extends. The basic system component type declaration forms the core for two type extensions, basic_plus and control. Within the extensions, the data input port declaration input_data is completed with a data type, and an additional port is added.

A more detailed discussion of the extension and refinement capabilities and additional example patterns is presented in Section 12.2 (Design Patterns). 
Section 4: AADL System Models and Specifications

Table 4-6: A Simple Extends and Refines Example

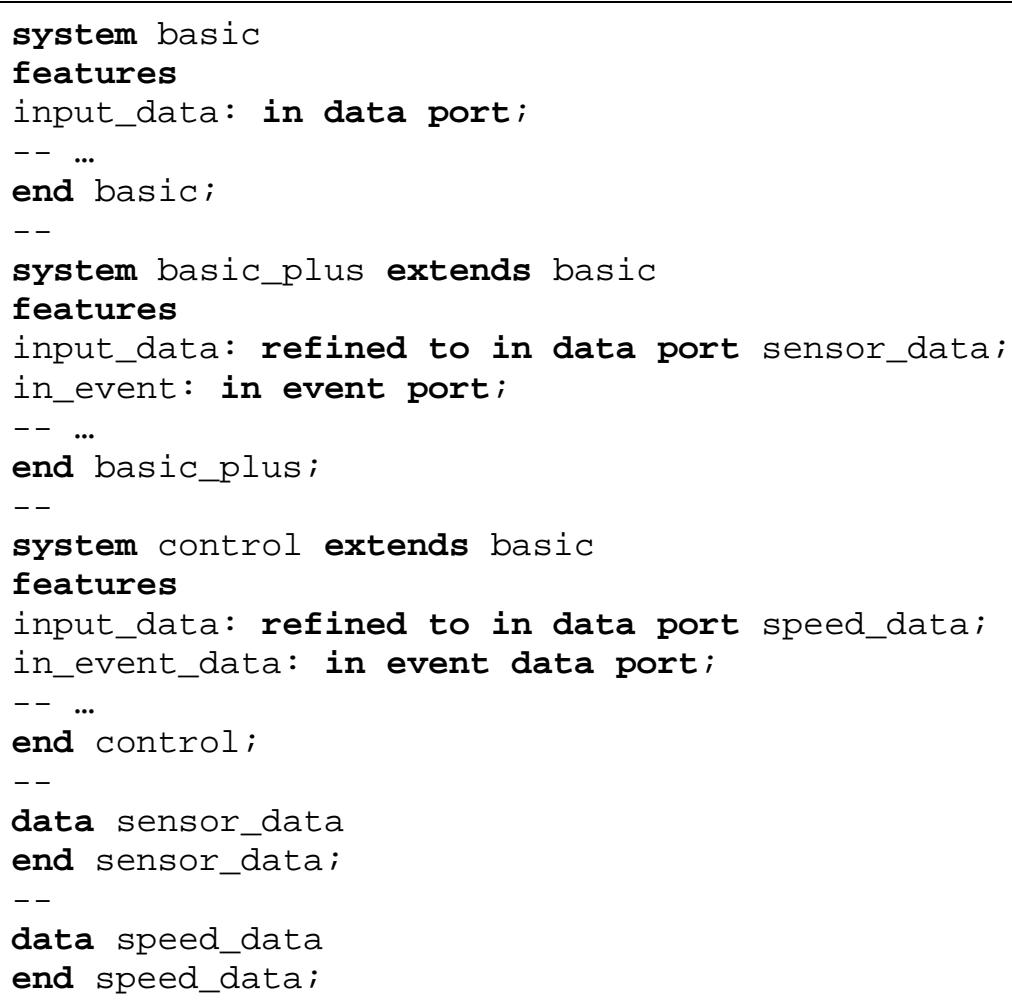




\section{Software Components}

Software component abstractions represent processed source text (executable binary images) and execution paths through executable code. Executable binary images (i.e., executable code and data) are the result of processing (such as compiling or linking) source text associated with a component. A component's source text may be written in a conventional programming language (e.g., Ada, Java, or C), domain-specific modeling language (e.g.,

MatLab/Simulink), or hardware description language (e.g., VHDL). The source text may also be an intermediate product of processing those representations (e.g., an object file).

The AADL software component abstractions are

- process (Section 5.1): represents a protected address space

- thread (Section 5.2): represents a unit of concurrent execution

- thread group (Section 5.3): represents a compositional unit for organizing threads

- data (Section 5.4): represents data types and static data in source text

- subprogram (Section 5.5): represents callable sequentially executable code

\subsection{Process}

The process abstraction represents a protected address space, a space partitioning where protection is provided from other components accessing anything inside the process. The address space contains

- executable binary images (executable code and data) directly associated with the process

- executable binary images associated with subcomponents of the process

- $\quad$ server subprograms (executable code) and data that are referenced by external components

A process does not have an implicit thread. Therefore, to represent an actively executing component, a process must contain a thread.

\subsubsection{Textual Representation}

Table 5-1 contains a partial listing of the textual specification for a process. The process is shown with examples of all three of its allowed subcomponent categories: (1) thread, (2) thread group, and (3) data. In this listing, simplified type and implementation declarations for the components are provided. Two ports are shown, one as input and one as output for the process. In a complete specification, connections that define the 
information flow would be declared within the process implementation. Only the subcomponent declarations of the process implementation of

control_processing.speed_control are shown explicitly. Other details of the specification are not included. These omissions are legal for a syntactically correct partial specification as discussed in Section 4.10 (Partial Specifications).

Table 5-1: Textual Representation of a Sample Process

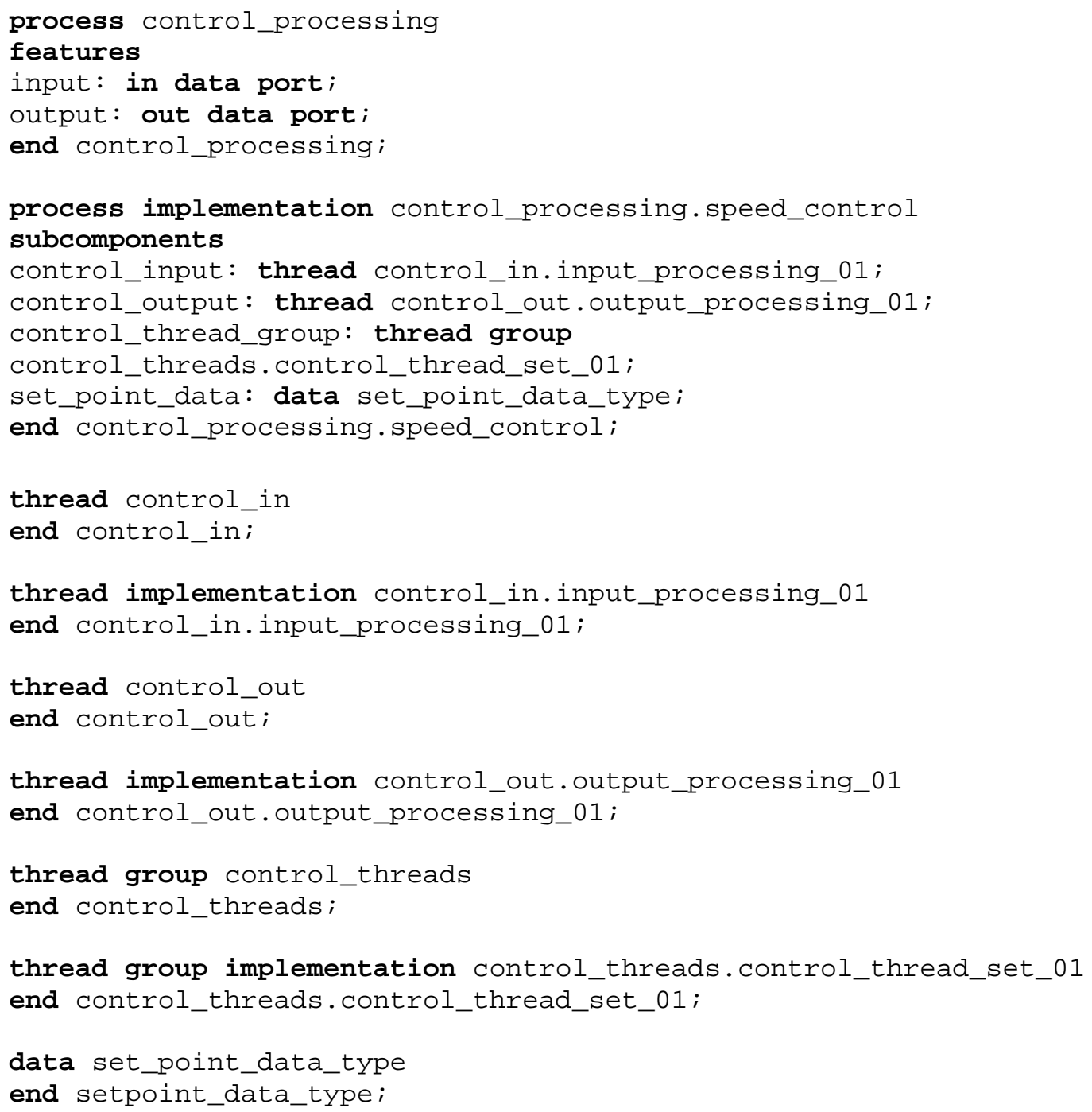

\subsubsection{Graphical Repesenation}

A graphical representation of the process implementation from Table 5-1 control_processing.speed_control is shown in Figure 5-1. The process is shown with examples of its allowed subcomponent categories: thread, thread group, and data. As shown in Figure 5-1, two threads (control_input and control_output), a single data component (set_point_data), and a thread group (control_thread_group) are contained within the process implementation control_processing.speed_control. 


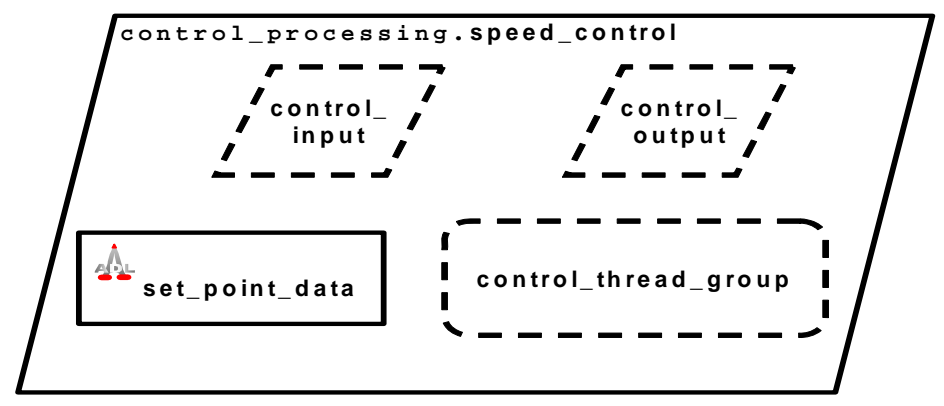

Figure 5-1: Graphical Representation of a Sample Process

\subsubsection{Properties}

For the process and its subcomponent threads, predeclared properties for processes enable the specification of the

- runtime enforcement of memory protection

- relevant source file information

- $\quad$ source file loading times

- scheduling protocols

- binding constraints

In addition, there are properties that can be inherited and shared by a process's subcomponent threads (e.g., Period, Deadline, or Actual_Processor_Binding). These include predeclared properties as well as new properties, defined as prescribed in Section 11.3 (Defining New Properties). ${ }^{12}$

\subsubsection{Constraints}

An AADL process represents only a protected address space. Consequently, processes must contain at least one explicitly declared thread or thread group subcomponent. In other words, it is not equivalent to a POSIX process that represents both a protected address space and an implicit thread.

Table 5-2 summarizes the permitted type declaration and implementation declaration elements of a process. A process can only be a subcomponent of a system component. A summary of the allowed subcomponent relationships and features is included in the Appendix on pages 117-119.

12 There is a standard predeclared property set named AADL_Properties that is a part of every AADL specification [SAE 06a]. 
Table 5-2: Summary of Permitted Process Declarations

\begin{tabular}{|c|c|c|}
\hline Category & Type & Implementation \\
\hline process & $\begin{array}{l}\text { Features: } \\
\text { - } \quad \text { server subprogram } \\
\text { - } \quad \text { port } \\
\text { - } \quad \text { provides data access } \\
\text { - } \quad \text { requires data access } \\
\text { Flow specifications: yes } \\
\text { Properties yes }\end{array}$ & $\begin{array}{l}\text { Subcomponents: } \\
\text { - data } \\
\text { - thread } \\
\text { - thread group } \\
\text { Subprogram calls: no } \\
\text { Connections: yes } \\
\text { Flows: yes } \\
\text { Modes: yes } \\
\text { Properties yes }\end{array}$ \\
\hline
\end{tabular}

\subsection{Thread}

A thread is a concurrent schedulable unit of sequential execution through source code. Multiple threads represent concurrent paths of execution. A variety of execution properties can be assigned to threads, including timing (e.g., worst case execution times), dispatch protocols (e.g., periodic, aperiodic, etc.), memory size, and processor binding.

\subsubsection{Textual Representation}

Sample thread type, implementation, and subcomponents declarations are shown in Table 5-3. In Table 5-3, there are two thread type and three thread implementation declarations. Two of the thread implementation declarations describe separate implementations of the same thread type data_input. Instances of threads are defined in subcomponents subclause declarations of the process implementation data_management.

Related type and implementation declarations are grouped together in this example. This grouping of declarations is used for clarity and is not a required organization within a specification. 
Table 5-3: A Sample Thread Declaration

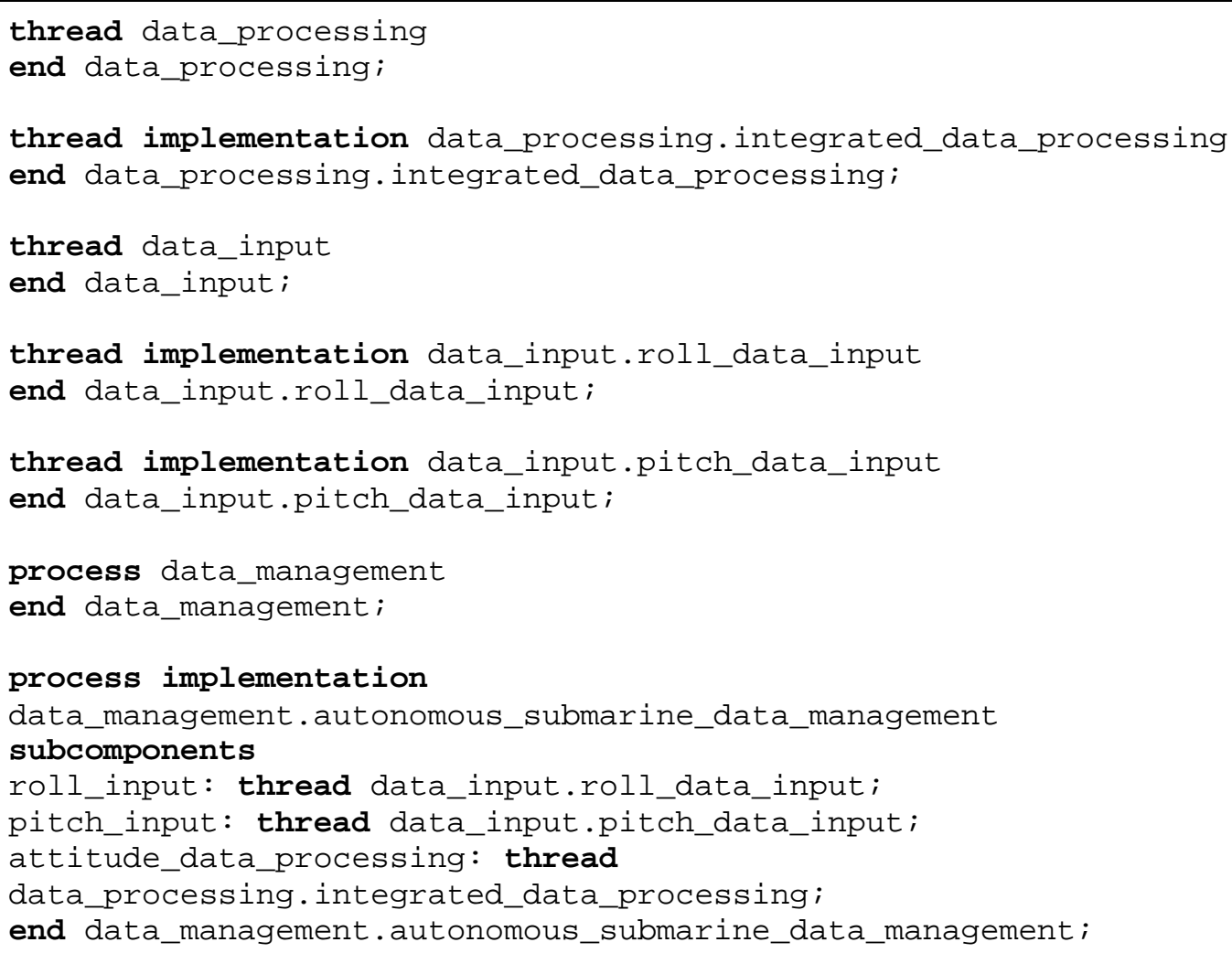

\subsubsection{Graphical Representation}

A graphical representation of the thread implementation

control_laws.control_input and its associated textual representation are shown in Table 5-4. No interfaces for the type or other details of the type or implementation declarations are shown.

In the example, the data instance configuration_data is defined as a subcomponent of the thread, and the referenced identifier is a data type rather than a data implementation. This is legal only if there are no implementation declarations of the data type anywhere within the specification. 
Table 5-4: A Sample Thread Implementation with One Subcomponent

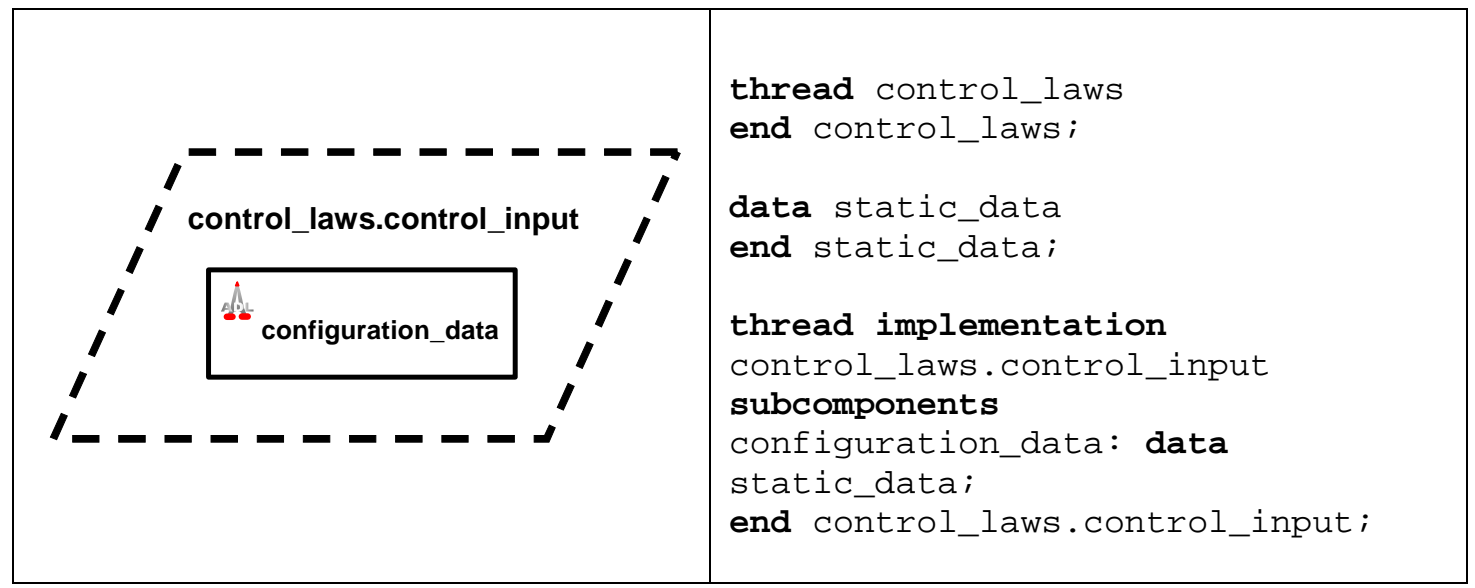

\subsubsection{Thread Execution}

A graphical state machine representation of thread execution is shown in Figure 5-2. A round-cornered rectangle represents an execution state of a thread or a composite state that includes at least one execution state. The ovals are non-execution states. Transitions between states are represented by directed arcs. Arcs may originate, join, diverge, or terminate at junction points depicted as small circles.

Instances of a thread can transition between various scheduling states as the result of normal execution (e.g., preemption or completion of initialization) or faults/errors. There are predefined entry points for each of the thread execution states: Initialize, Compute, and Recover. The initialize and compute entry points are used for normal execution.

If thread execution results in a fault that is detected, the source text may handle the error. If the error is not handled in the source text, the thread is requested to recover and prepare for the next dispatch. If an error is considered unrecoverable, its occurrence is propagated as an event through the thread's predeclared out event data port Error (not shown in Figure 5-2). All threads have an Error out event data port that allows an unrecoverable error with descriptive information to be signaled. 


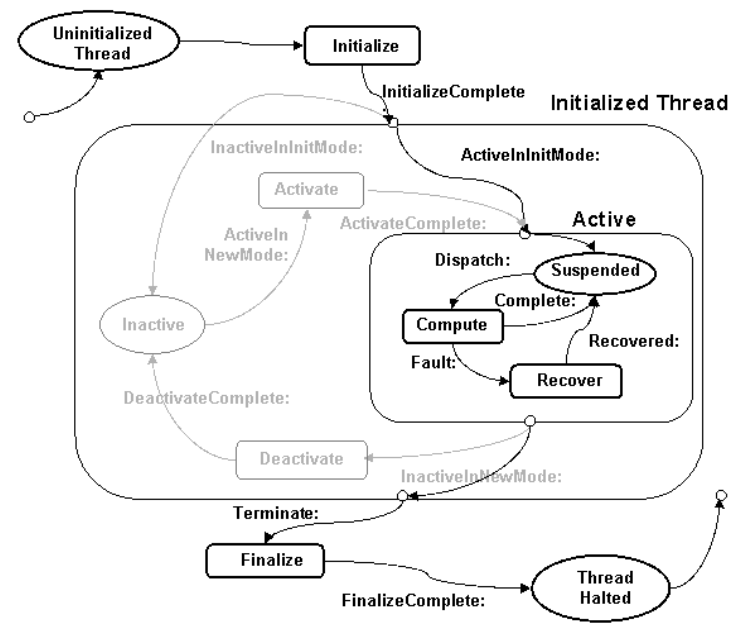

Figure 5-2: $\quad$ Thread Execution State Machine

\subsubsection{Properties}

Predeclared properties support the detailed description of each of the execution phases of a thread. There are entry point properties that specify entry into code associated with each of these execution phases (Figure 5-2):

1. Initialize allows threads to perform application specific initialization.

2. Activate allows actions to restore application states between mode switches.

3. Compute represents the code to be executed on every thread dispatch.

4. Recover allows threads to perform fault recovery actions.

5. Deactivate allows actions to save application states between mode switches.

6. Finalize executes when thread is asked to terminate as part of a process unload or stop. In addition, there are execution time and deadline properties for each of these execution phases (not shown in Figure 5-2).

A thread's scheduling-related properties include Dispatch_Protocol and Period. The supported protocols are

- periodic: repeated dispatches occurring at a specified time interval (a Period)

- aperiodic: event-triggered dispatch of threads

- sporadic: event-driven dispatch of threads with a minimum dispatch separation

- background: a dispatch initiated once with execution until completion

Periodic, aperiodic, and sporadic protocols typically involve hard deadlines for the thread. The predeclared and user-defined thread properties can be used to specify critical runtime aspects of a thread within a system's architectural representation, enabling the early analyses of thread behavior. 
Table 5-5 is an example of some property associations for a thread. Entry points and associated execution times are declared for initialization and nominal execution.

Table 5-5: Sample Thread Properties

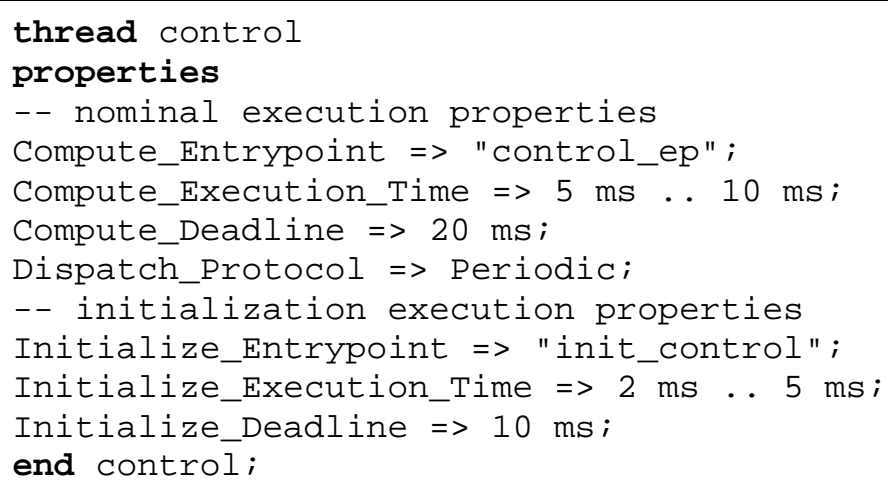

\subsubsection{Constraints}

Table 5-6 summarizes the legal subclause declarations for a thread.

Table 5-6: Summary of Permitted Thread Subclause Declarations

\begin{tabular}{|c|l|l|}
\hline Category & \multicolumn{1}{|c|}{ Type } & \multicolumn{1}{|c|}{ Implementation } \\
\hline \multirow{5}{*}{ thread } & Features: & Subcomponents: \\
& $\bullet \quad$ server subprogram & data \\
& $-\quad$ port & Subprogram calls: yes \\
& $-\quad$ provides data access & Flows: yes \\
& Flow specifications: yes & Modes: yes \\
& Properties yes & Properties yes \\
\hline
\end{tabular}

A thread executes within the protected virtual address space of a process, either as an explicitly declared subcomponent or as a subcomponent of a thread group within a process. Thus, threads must be contained within (i.e., only be a subcomponent of) a process or a thread group. Multiple concurrent threads can exist within a process.

A summary of the allowed subcomponent relationships and features is included on pages 117-119 in the Appendix. 


\subsection{Thread Group}

A thread group is a component abstraction for logically organizing thread, data, and thread group components within a process. Thread groups do not represent a virtual address space or a unit of execution. They provide a foundation for the separation of concerns in the design, defining a single reference to multiple threads and associated data (e.g., threads with a common execution rate or all threads and data components needed for processing input signals).

\subsubsection{Textual Representation}

Table 5-7 is a sample textual specification for a thread group that contains a thread component, two data components, and another thread group. Simplified thread group type and implementation declarations are shown. For example, only the subcomponents declarations part of the control.roll_axis component implementation declaration is shown. No details of the thread group implementation control_laws.roll are shown. Notice that the data subcomponent declarations control_data and error_data reference data implementation declarations rather than data type declarations, reflecting the flexibility that static data components can be declared at any level of the hierarchy. The thread group type declaration for control includes a property association that defines a Period of $50 \mathrm{~ms}$. This value is assigned to (inherited by) all of the threads contained in the thread group.

Table 5-7: A Sample Thread Group AADL Textual Specification

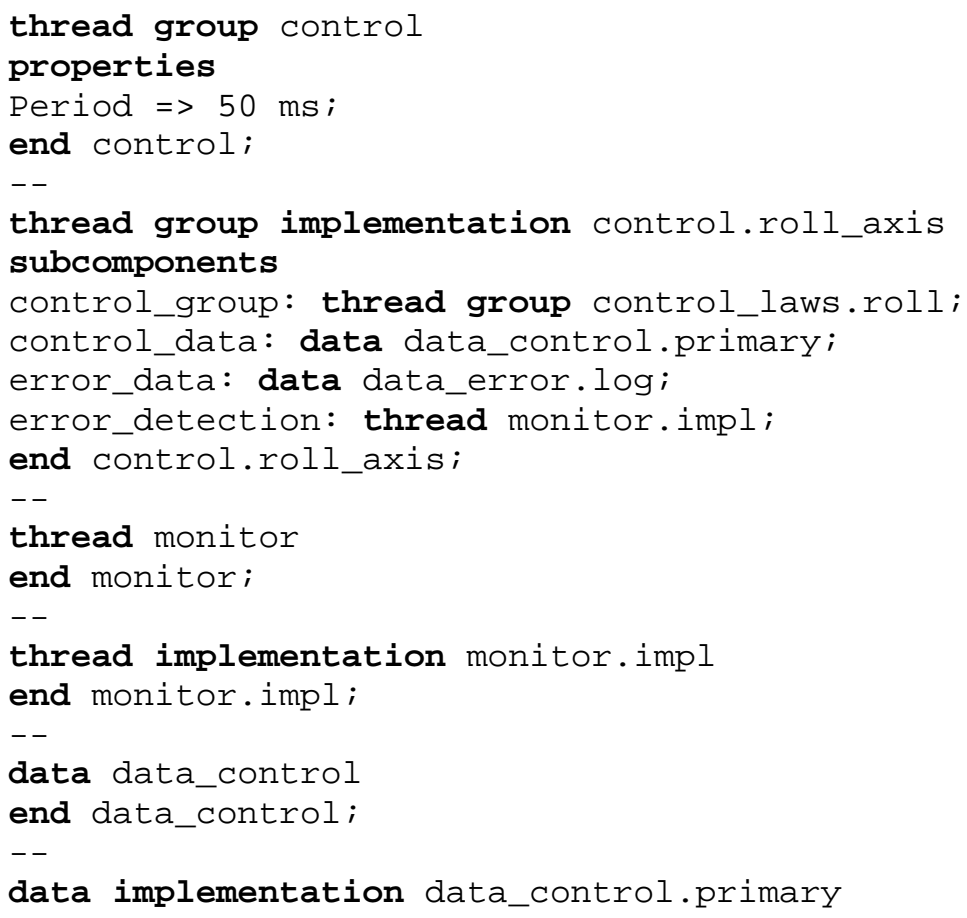


Table 5-7: A Sample Thread Group AADL Textual Specification

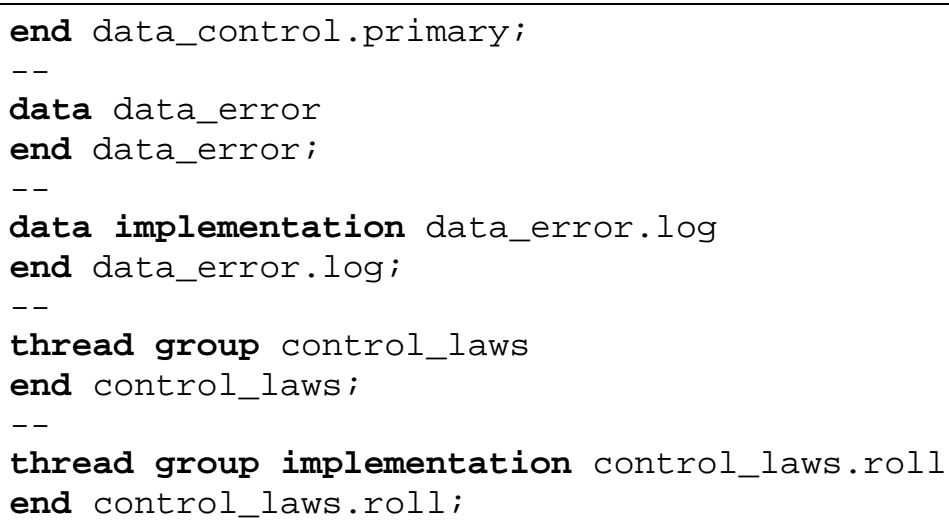

\subsubsection{Graphical Representation}

Figure 5-3 contains a graphical representation of the implementation of the thread group control.roll_axis shown textually in Table 5-7. Notice that the names (identifiers) of the graphical subcomponents of the thread group match those contained in the textual representation of the thread group's implementation declaration. Partial declarations are permitted in the initial specification of the system (e.g., subcomponent declarations may not have component type or implementation references). This partial specification capability is particularly useful during early design stages where details may not be known or decided. Component classifier references can be added or completed in subcomponent refinements or declared in component implementation extensions. For example, in Table 5-7 the declaration for the subcomponent error_detection does not have to include the thread component classifier monitor . impl. This reference could be added later in an extension of the thread group implementation control.roll_axis.

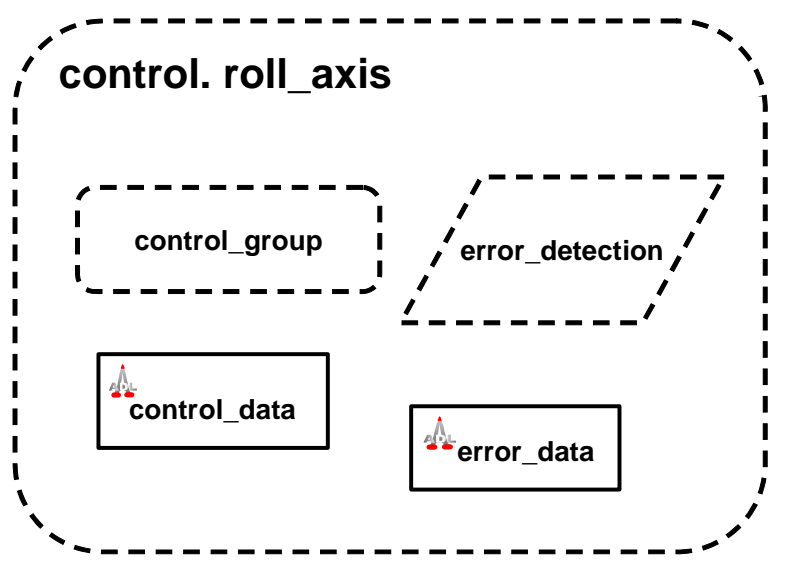

Figure 5-3: $\quad$ A Sample Thread Group Graphical Representation 


\subsubsection{Properties}

Predeclared thread group properties include declarations relating to the specification of

- $\quad$ source text

- timing characteristics

- relevant memory, processor, and connection bindings ${ }^{13}$

For example, there are Actual and Allowed_Processor_Binding properties for threads within the thread group, as well as properties that describe thread handling during mode changes (e.g., Active_Thread_Handling_Protocol that specifies the protocol to use for execution at the time instant of an actual mode switch). ${ }^{14}$

\subsubsection{Constraints}

A thread group can be a subcomponent only of a process or another thread group. Table 5-8 summarizes the permitted elements of a thread group's type and implementation declarations.

Table 5-8: Elements of a Thread Group Component

\begin{tabular}{|l|l|l|}
\hline Category & \multicolumn{1}{|c|}{ Type } & \multicolumn{1}{|c|}{ Implementation } \\
\hline & Features: & Subcomponents: \\
& $\bullet \quad$ server subprogram & - data \\
thread & $\bullet \quad$ port & thread \\
group & $\bullet \quad$ provides data access & thread group \\
& Flow specifications: yes & Connections: yes \\
& Froperties yes & Flows: yes \\
& & Modes: yes \\
& & Properties yes \\
\hline
\end{tabular}

A summary of the allowed subcomponent relationships and features is included on pages 117-119 in the Appendix.

13 The mapping of software to hardware components of a system that are required to produce a physical system implementation is called binding [SAE 06a].

14 Actual_Processor_Binding, Allowed_Processor_Binding, and Active_Thread_Handling_Protocol are predeclared properties in the standard predeclared property set AADL_Properties. 


\subsection{Data}

The AADL data abstraction represents static data (e.g., numerical data or source text) and data types within a system. Specifically, data component declarations are used to represent

- application data types (e.g., used as data types on ports and parameters)

- the substructure of data types via data subcomponents within data implementation declarations

- data instances

Data types in the application system can be modeled by data component type and implementation declarations. A data type (and implementation) declaration can be used to define the data associated with ports and parameters. It is sufficient to model an application source text data type with a data component type and relevant property declarations; it is not necessary to declare a data implementation. Consistency checks can be done on the data type associated with connections between ports and parameters. Data subcomponent declarations can be used to define the substructure of data types and instances. For example, fields of a record can be declared as data subcomponents in a data implementation declaration.

Data instances are represented by data subcomponent declarations within a software component or system implementation. Currently data subcomponents cannot be declared in subprograms. For example, data instances within source text (e.g., the instance variables of a class or the fields of a record) are represented by data subcomponent declarations in a data component implementation. These data instances can be declared as accessible by multiple components as outlined in Section 8.3 (Subcomponent Access). Data component types can have subprograms as features, allowing for modeling of abstract data types or classes with access methods.

\subsubsection{Textual Representation}

Sample data type and implementation declarations are shown in Table 5-9 that includes three data type declarations and a data implementation declaration address . others of the data type declaration address. In addition, a thread implementation declaration is shown with data subcomponents that reference the data types defined in Table 5-9.

As the commented description in the table explains, the first part of the table shows the data type string used in a port declaration. Specifically, it shows the declaration of a data type speed_data_type used to declare the data type for an input data port of the process controller. The property association defines the size of the data type as 16 bits. Only relevant portions of the controller process type declaration are included. The second part of the table shows an example of the declaration of the substructure of a data implementation. The substructure of the data implementation 
address . others consists of four data subcomponents with data types string and int. In the third and final portion of the table, the thread implementation declaration for address_processing.address_lookup includes a specific data instance of the data implementation address. others as a subcomponent.

Notice that the data subcomponent declarations within the data implementation address_others reference only the data type declaration. Subcomponents subclauses can reference a data type declaration rather than a data implementation declaration only if there is no more than one implementation of that data type.

Table 5-9: Sample Data Component Declarations

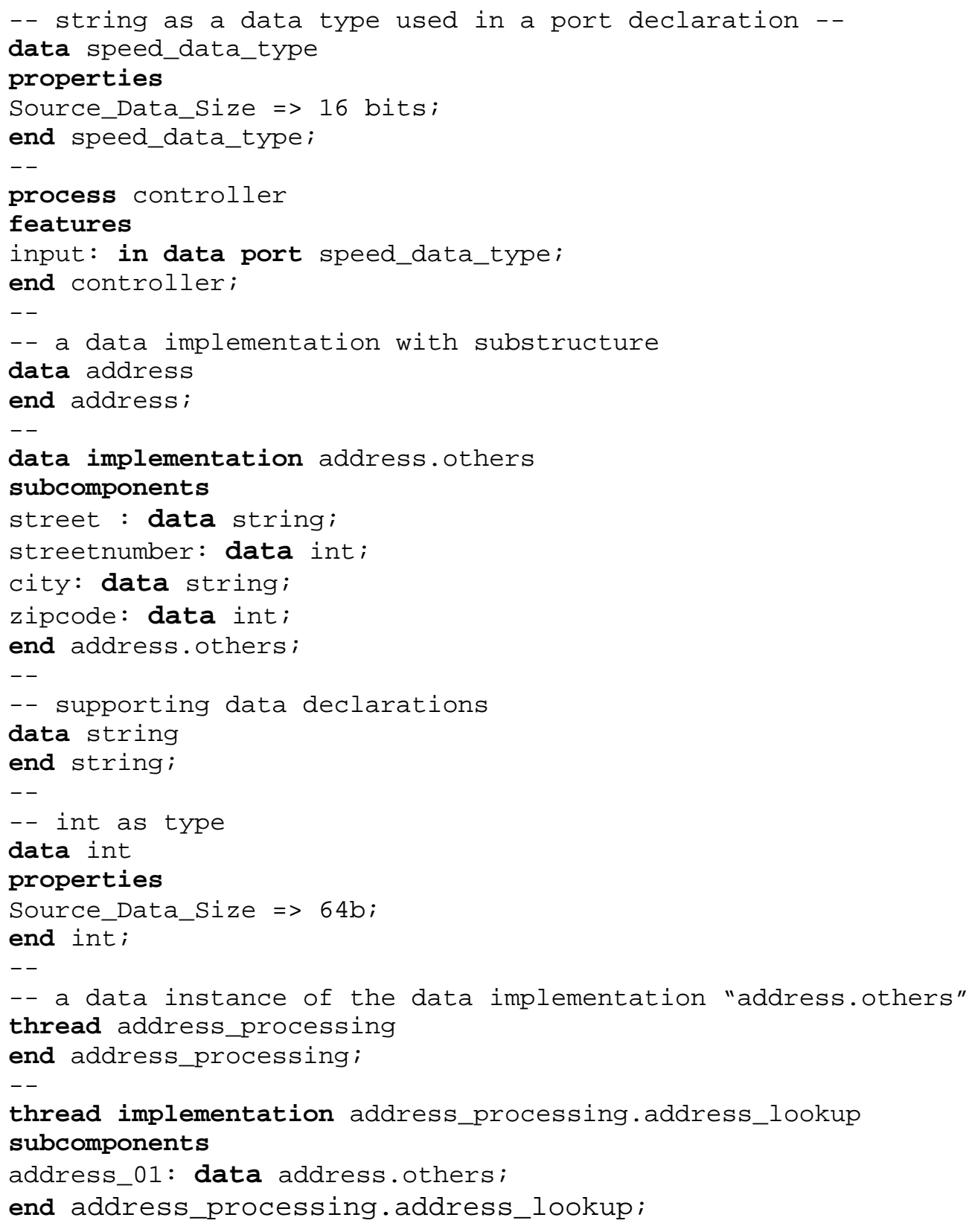




\subsubsection{Graphical Representation}

Figure 5-4 contains graphical and corresponding textual representations for the data subcomponents of the data implementation address . others and the thread implementation address_processing.address_lookup presented in

Table 5-9.
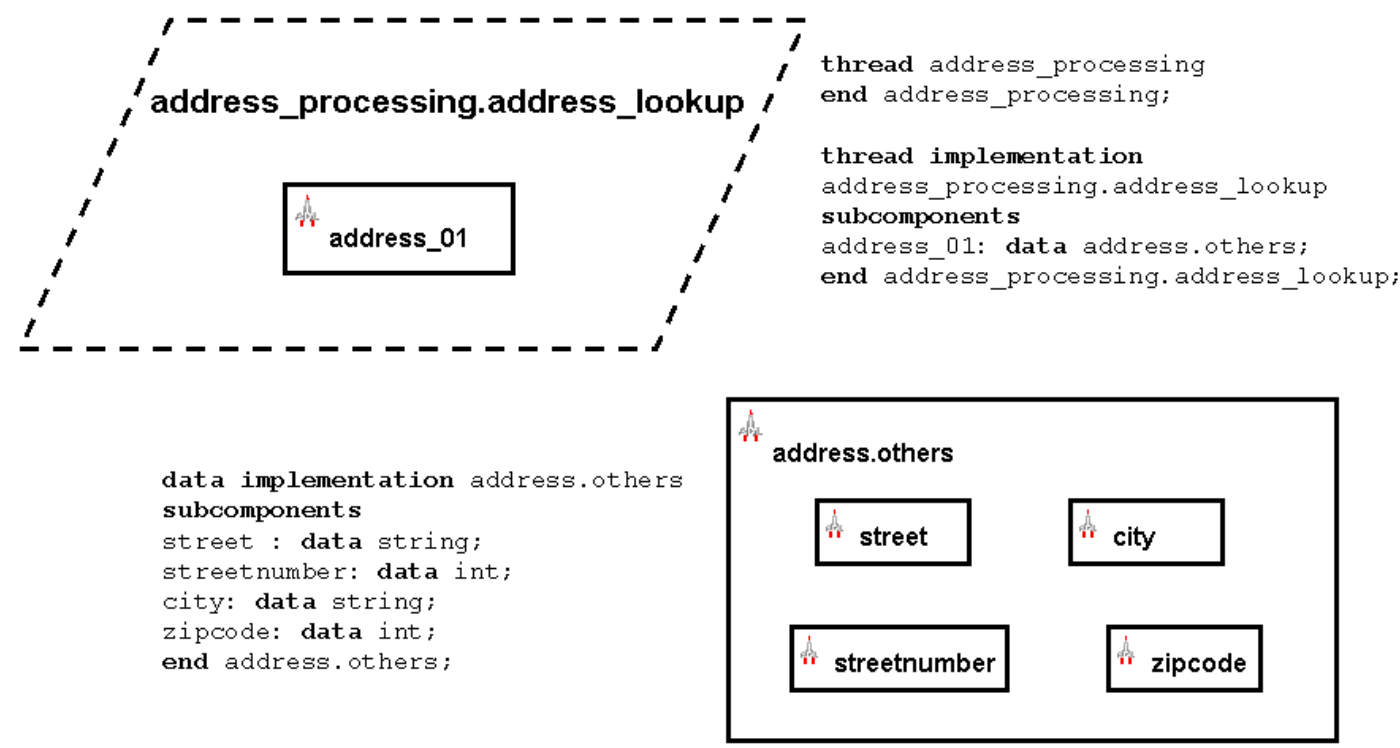

Figure 5-4: $\quad$ Sample Data Component Graphical Representations

\subsubsection{Properties}

The predeclared properties for data components enable specification of

- $\quad$ source text for the data component

- name of the relevant data type declaration

- name of the relevant static data variable in the source text

- data size

- concurrency access protocol for shared data

Base types can be modeled using data types by

1. defining a new property (such as BaseType) that takes a (data) classifier as property value

2. applying this property to data components

3. declaring data component base types (such as SignedInt16 or UnsignedInt8) 
For example, BaseType => classifier BaseTypes: : SignedInt16; could be a property declared in the data type speed_data_type, where the data type SignedInt16 is declared in the package BaseTypes.

\subsubsection{Constraints}

Table 5-10 summarizes the legal elements within data type and data implementation declarations. Notice that only data components can be subcomponents within a data component.

A data component can be a subcomponent of a data, thread, thread group, process, or system component. A summary of the allowed subcomponent relationships and features is included on pages 117-119 in the Appendix.

Table 5-10: Legal Elements of Data Type and Implementation Declarations

\begin{tabular}{|c|l|l|}
\hline Category & \multicolumn{1}{|c|}{ Type } & \multicolumn{1}{c|}{ Implementation } \\
\hline \multirow{4}{*}{ data } & Features: & Subcomponents: \\
& $\bullet \quad$ subprogram & - data \\
& Flow specifications: no & Subprogram calls: no \\
& Properties yes & Connections: access \\
& & Flows: no \\
& & Modes: yes \\
& & Properties yes \\
\hline
\end{tabular}

A data subcomponent subclause can reference a data type declaration that does not have a data implementation. For example, the reference for the subcomponent street of the data implementation address . others shown in Figure 5-4 is to the data type string. However, if a data type declaration has more than one associated data implementation declaration, both the component type and a component implementation must be present in a component classifier reference in order to completely identify the classifier.

\subsection{Subprogram}

The subprogram component abstraction represents sequentially executable source text- $\mathrm{a}$ callable component with or without parameters that operates on data or provides server functions to components that call it. A subprogram and its parameter signature are declared through component declarations but are not instantiated as subcomponents. Instead, calls to subprograms are declared in calls sequences in thread and subprogram implementations. More details on calls to subprograms and example calls declarations are provided in Section 8.4 (Subprogram Calls). 
The modeling roles for subprograms include the representation of

- a method call for operation on data

- basic program calls and call sequencing

- remote service/procedure calls

These calls can include data transfer into or out of the subprogram. Parameters, declared as features of a subprogram, provide the interface for the transfer of data into or out of a subprogram.

\subsubsection{Textual Representation}

Table 5-11 is an example of a subprogram representing a service (method) call for operation on data. It shows the relevant component type and implementation declarations and the declaration of that subprogram as one of the features scale_acc_data within a data component accelerometer_data. The feature scale_acc_data represents an entry point into source text that operates on the data component accelerometer_data.

Table 5-11: $\quad$ Subprogram Textual Representation

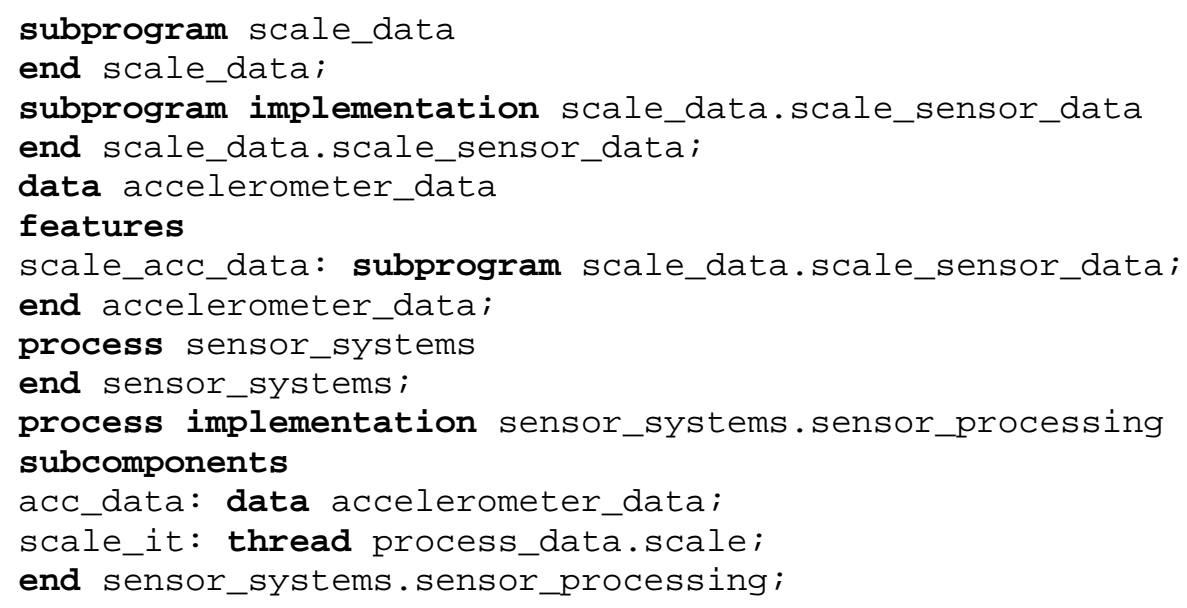

\subsubsection{Graphical Representation}

Figure 5-5 contains graphical and corresponding textual representations for the process implementation sensor_systems.sensor_processing shown in Table 5-11. The subprogram scale_acc_data is represented by an oval that adorns the data subcomponent acc_data of the process implementation sensor_systems.sensor_processing. The thread scale_it is not shown in the figure. 


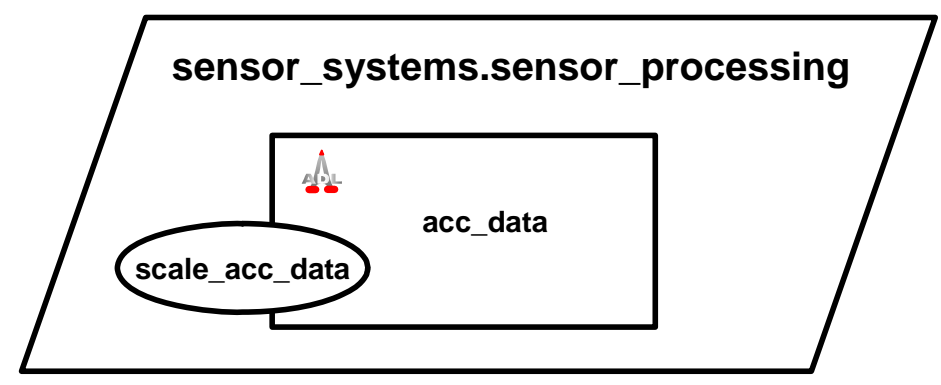

Figure 5-5:Subprogram Graphical Representation

Table 5-12 shows both textual (upper portion) and graphical (lower portion) representations of an example of a subprogram abstraction representing a server subprogram.

In this textual representation, the two process implementation declarations (control.temp_control and manage_data.manage_temp) are bound to separate memory components (e.g., memories associated with individual processing nodes on a distributed computing network). The thread implementation control_law.linear within the control.temp_control process implementation calls the subprogram acquire . temp that is declared as a server subprogram feature in the thread type read.

In the graphical representation of the specification shown in the lower portion of Table 5-12, the subroutine entry point read_it is identified as a feature of the subcomponent thread temp_reader. In addition, the call get_temp is shown in the thread control . temp_control, and the binding of this call to the read_it subprogram is shown with an arrowed line. This call can be a remote call, where the server

subprogram thread temp_reader is bound to a separate processor than the calling thread linear 01 . More details on subprogram calls and a remote clientserver example can be found in Section 8.4 (Subprogram Calls).

\section{Table 5-12: $\quad$ Example Textual and Graphical Subroutine Declarations}

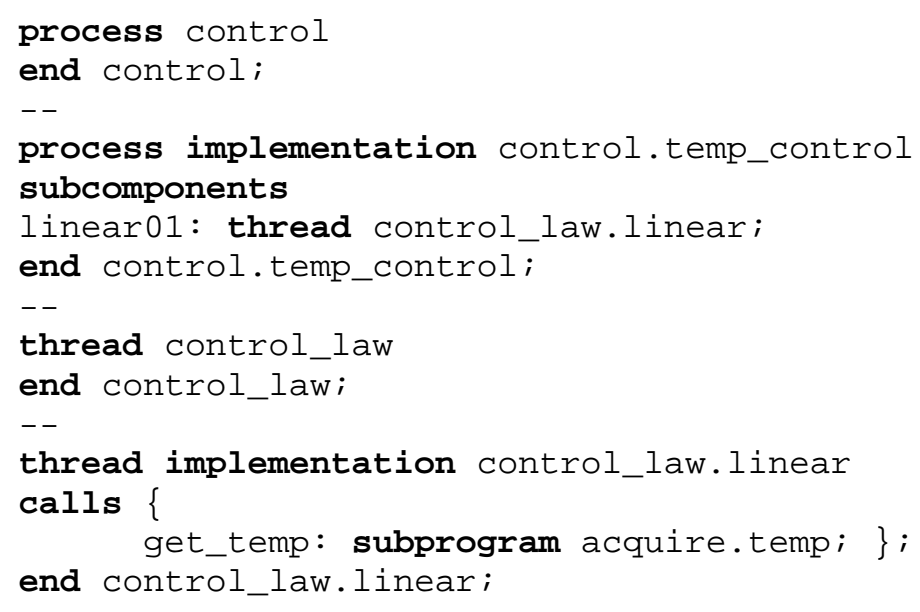


Table 5-12: $\quad$ Example Textual and Graphical Subroutine Declarations (cont.)
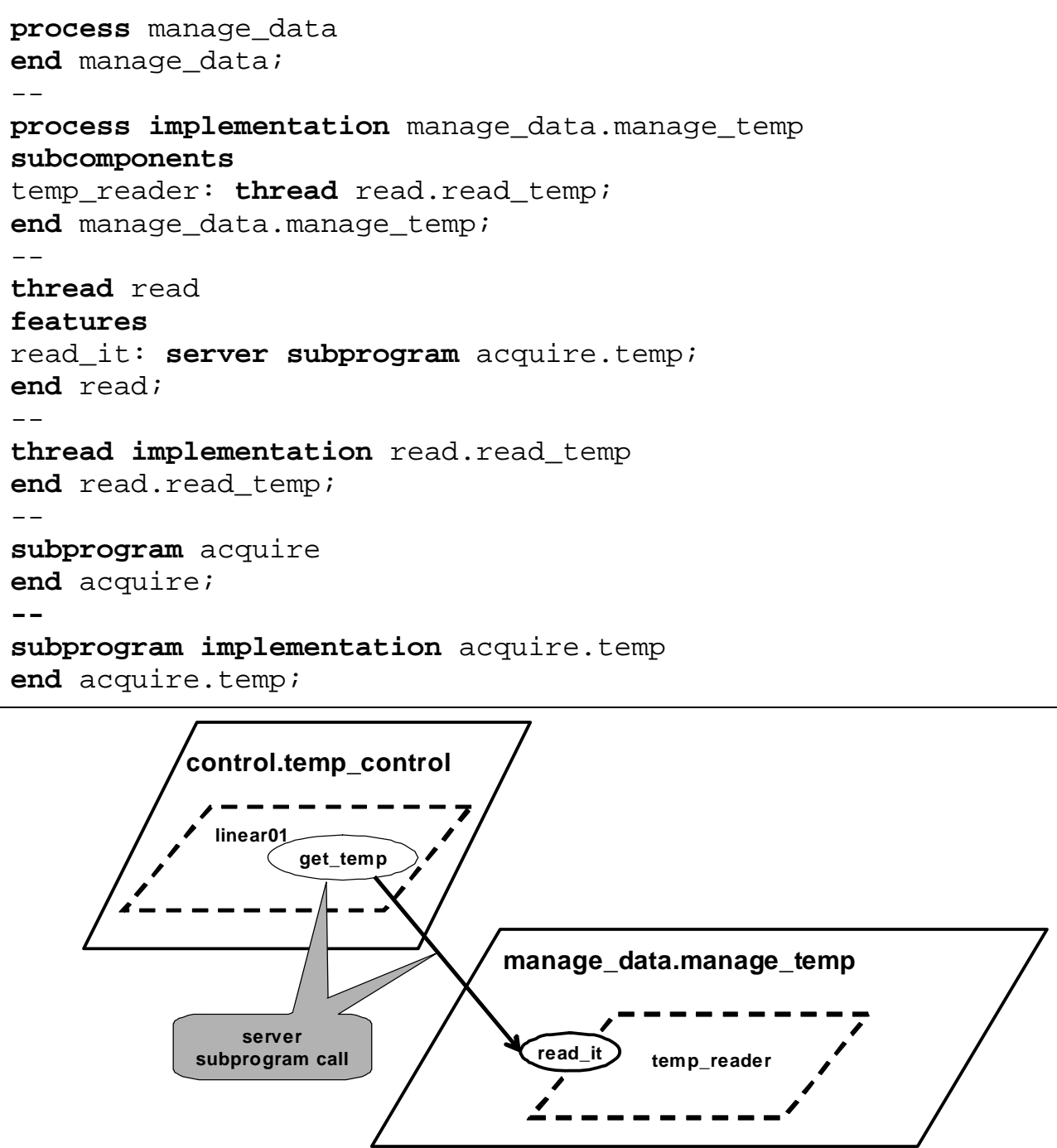

\subsubsection{Properties}

Predeclared subprogram properties include declarations relating to the

- $\quad$ source text for the subprogram

- memory requirements

- memory binding

- characteristics related to calls into the subprogram 


\subsubsection{Constraints}

Table 5-13 summarizes the permitted elements of a subprogram's component type and implementation declarations.

Table 5-13: Restrictions on Subprogram Declarations

\begin{tabular}{|c|l|l|}
\hline Category & \multicolumn{1}{|c|}{ Type } & \multicolumn{1}{|c|}{ Implementation } \\
\hline & Features: & Subcomponents: \\
& $\bullet \quad$ out event port & none \\
subprogram & $-\quad$ out event data port & Subprogram calls: yes \\
& $-\quad$ port group & Connections: yes \\
& $-\quad$ requires data access & Flows: yes \\
& Flow specifications: yes & Modes: yes \\
& Properties yes & Properties yes \\
\hline
\end{tabular}

The interactions of subprograms are constrained to

- event-based interfaces: out event port, out event data port, and a port group consisting only of these event port types

- data interfaces: through parameters of calls to and returns from the subprogram

Out event ports and out event data ports support modeling subprograms that raise an event (with or without associated data) that must be passed through an enclosing thread to other components. A subprogram may require access to data but cannot contain static data subcomponents. 


\section{Execution Platform Components}

Execution platform components represent computational and interfacing resources within a system. This representation includes complex hardware and associated software systems. For example, in one model a Linux computing resource can be represented as a processor and, in an implementation model of the processor, as a system with Linux software mapped onto an execution platform processor.

There are four categories of execution platform components in the AADL:

1. processor (Section 6.1): represents components that execute threads

2. memory (Section 6.2): represents components that store data and code

3. bus (Section 6.3): represents components that provide access among execution platform components

4. device (Section 6.4): represents components that interface to the external environment

Within an AADL specification, software components must be mapped onto execution platforms through binding relationships. These bindings define where code is executed and data and executable code are stored within a system. For example, a thread must be bound to a processor for execution and a process must be bound to memory. Similarly, connections among components within a system must be bound to appropriate execution platform components (e.g., a simple connection is bound to a single bus or a connection within a complex distributed system is bound to a sequence of buses and intermediate processors and devices). Additional information on binding is in Section 7 (System Structure and Instantiation).

A collection of execution platform components contained within an AADL system abstraction can be used to model complex physical computational resources. For example, memory that represents a hard disk and a processor that supports software execution within a system can model a database server. Similarly, a collection of software and execution platform components (i.e., a system implementation) can represent a virtual machine layer within a layered system architecture model.

\subsection{Processor}

A processor is an abstraction of hardware and associated software that is responsible for scheduling and executing threads. Processors can execute threads that are declared in application software systems or threads that reside in components accessible from those processors. 
Processors themselves may have embedded software (e.g., an operating system) that implements scheduling and other capabilities that support thread execution. Alternatively, separate software components or other software virtual machines can supply this support, provided that software is bound to memory that is accessible by the processor.

\subsubsection{Textual and Graphical Representations}

Table 6-1 shows a type and implementation declaration for a processor. Both textual and corresponding graphical representations are shown. In this example, a single processor system with memory contained inside of the processor is shown. No other interconnections are required.

Table 6-1: A Sample Processor Textual and Graphical Representation

\begin{tabular}{|c|c|}
\hline $\begin{array}{l}\text { processor Intel_Linux } \\
\text { properties } \\
\text { Hardware_Source_Language=> VHDL; } \\
\text { Hardware_Description_Source_Text => } \\
\text { "intel_vhdl_1,_intel_vhdl_2"; } \\
\text { end Intel_Linux; } \\
\text {-- } \\
\text { processor implementation } \\
\text { Intel_Linux. Intel_Linux_01 } \\
\text { subcomponents } \\
\text { HSRAM: memory RAM. Intel_RAM; } \\
\text { end Intel_Linux.Intel_Linux_01; } \\
- \text {-- } \\
\text { memory RAM } \\
\text { end RAM; } \\
\text {-- } \\
\text { memory implementation RAM. Intel_RAM } \\
\text { end RAM.Intel_RAM; }\end{array}$ & $\begin{array}{c}\text { InteI_Linux.Intel_Linux_01 } \\
\text { ImSRAM } \\
\text { Implementation }\end{array}$ \\
\hline
\end{tabular}

In the textual representation, the properties subclauses define the hardware description language (Hardware_Source_Language) and the files that contain the source text for the hardware description (Hardware_Description_Source_Text). The

processor implementation declaration of Intel_Linux. Intel_Linux_01 includes a single memory subcomponent HSRAM. The memory subcomponent's type and implementation declarations are shown.

The corresponding graphical representations of type and implementation are shown to the right of the textual representation in Table 6-1. The nesting of the memory graphic (labeled HSRAM) within the processor graphic shows containment. The optional bold line (discussed in Section 4.3) is not used for the processor implementation graphic. 


\subsubsection{Properties}

Predeclared processor properties can be used in a processor declaration. In addition to the hardware description properties included in the example from Table 6-1, other properties include a Scheduling_Protocol property that must have a value if threads are bound to the processor and an Allowed_Dispatch_Protocol property that specifies the dispatch protocols supplied by the processor. ${ }^{15}$

\subsubsection{Constraints}

Table 6-2 summarizes the permitted elements of a processor's type and implementation declarations.

Table 6-2: Summary of Permitted Processor Declarations

\begin{tabular}{|l|l|l|}
\hline \multicolumn{1}{|c|}{ Category } & \multicolumn{1}{|c|}{ Type } & \multicolumn{1}{|c|}{ Implementation } \\
\hline \multirow{5}{*}{ processor } & Features: & Subcomponents: \\
& $-\quad$ server subprogram & - memory \\
& $-\quad$ port group & Subprogram calls: no \\
& - requires bus access & Connections: no \\
& Flow specifications: yes & Modes: yes \\
& Properties yes & Properties yes \\
\hline
\end{tabular}

A processor can only be a subcomponent of a system component. A summary of the allowed subcomponent relationships and features is included on pages 117-119 in the Appendix.

\subsection{Memory}

Memory abstractions represent storage components for data and executable code (i.e., subprograms, data, and processes are bound to memory components). Memory components include randomly accessible physical storage (e.g., RAM, ROM) or complex permanent storage such as disks or reflective memory. Since they have a physical runtime presence, memory components have properties such as word size and word count.

The memory component can represent memory inside of a processor or a separate execution platform unit that must be connected to a processor through a bus. Memory banks can be modeled as a single or composite memory unit.

15 There is a standard predeclared property set named AADL_Properties that is a part of every AADL specification [SAE 06a]. 


\subsubsection{Textual and Graphical Representations}

An example memory declaration and its graphical representation are shown in Table 6-3. In this example, a memory of the type RAM is declared with a single feature bus 01 that establishes that all instances of RAM require access to the bus membus. hsbus. No explicit properties for this type are declared. The type and implementation declarations for the requires bus access to bus 01 are shown at the end of the listing.

The memory implementation RAM . compRAM declares that this implementation of the memory type RAM includes memory subcomponents HSRAMO1 and SRAMO1. No modes or properties are declared. The subcomponents of the memory implementation RAM . compRAM are declared as implementations of a common type XRAM. An expanded memory composition can be used to model a complicated memory bank. These examples show that memory can only contain other memory components and must be connected to a bus unless it is enclosed in a processor.

Table 6-3: A Sample Memory Textual and Graphical Representation

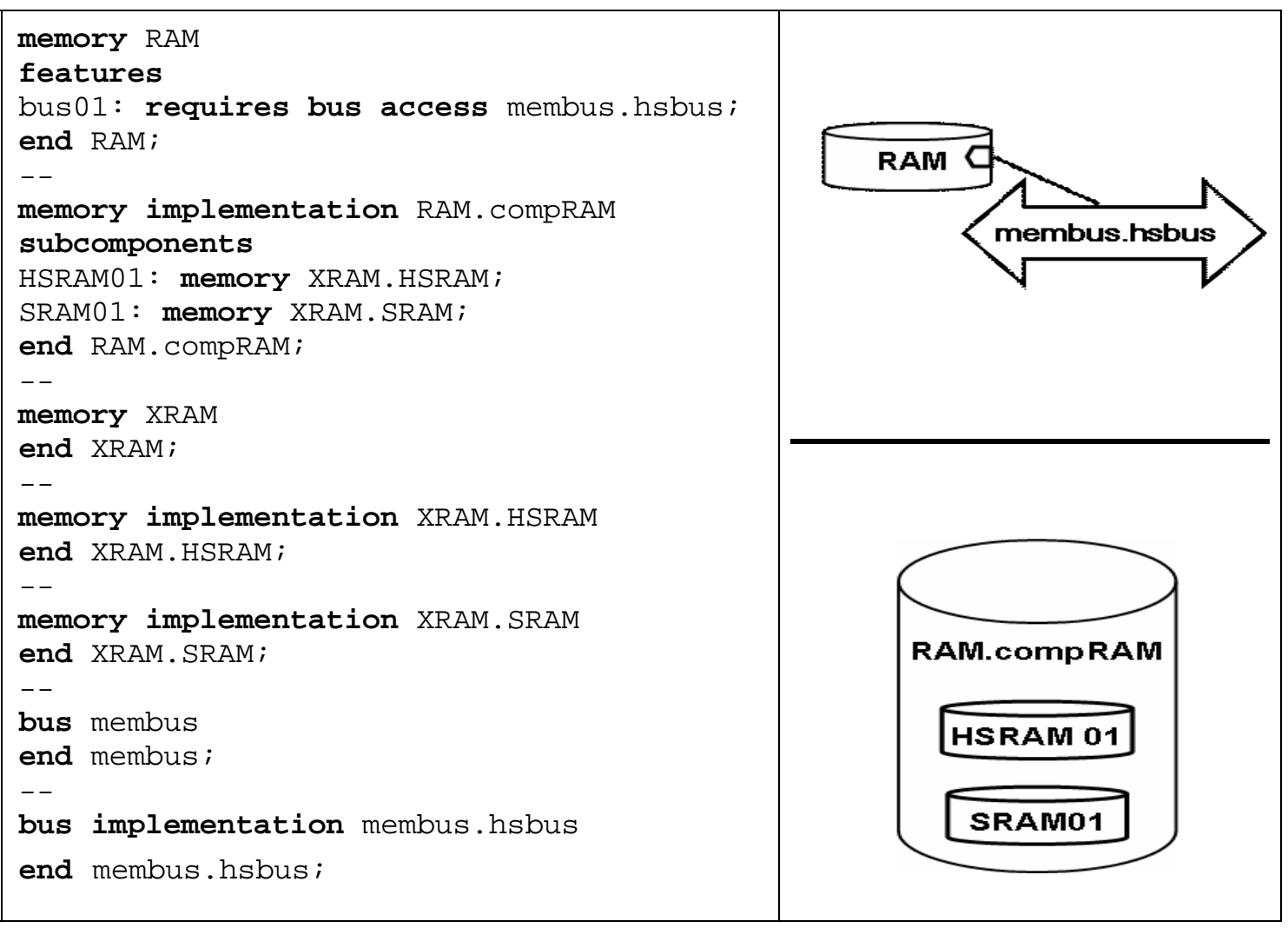




\subsubsection{Properties}

Predeclared memory properties include

- memory access protocol

- $\quad$ word size

- other important descriptive characteristics of storage units

- The default value for memory access (Memory_Protocol) is read-write but can be associated with the values of read only or write only.

\subsubsection{Constraints}

Table 6-4 lists the permitted elements of memory type and implementation declarations.

Table 6-4: Summary of Permitted Memory Declaration Subclauses

\begin{tabular}{|c|c|c|}
\hline Category & Type & Implementation \\
\hline memory & $\begin{array}{l}\text { Features } \\
\text { - requires bus access } \\
\text { Flow specifications: no } \\
\text { Properties yes }\end{array}$ & $\begin{array}{l}\text { Subcomponents: } \\
\text { - memory } \\
\text { Subprogram calls: no } \\
\text { Connections: no } \\
\text { Flows: no } \\
\text { Modes: yes } \\
\text { Properties yes }\end{array}$ \\
\hline
\end{tabular}

A memory component can only be contained within a memory, processor, or system component. Moreover, an individual memory component must be contained in a processor, declared a subcomponent of a memory unit, or connected to a processor through a bus. A summary of the allowed subcomponent relationships and features is included on pages 117-119 in the Appendix.

\subsection{Bus}

A bus represents hardware and associated communication protocols that enable interactions among other execution platform components (i.e., memory, processor, and device). For example, a connection between two threads, each executing on a separate processor, is over a bus between those processors. This communication is specified using access and binding declarations to a bus. Buses can be connected directly to other buses to represent complex inter-network communications. Thus, connections between components can be bound to a sequence of buses or a sequence of buses with intervening processors. 


\subsubsection{Textual and Graphical Representations}

Since a bus acts only as a shared component, its interactions (features) are specified as bus access features in component type declarations. For example, a processor requires access to a bus in order to communicate with memory that contains the threads executing on that processor. Similarly, a bus may require access to another bus.

Alternatively, a system may provide access to one of its bus subcomponents.

Table 6-5 shows a portion of an AADL textual specification and its corresponding graphical representation. Included in the example are a processor type declaration for Intel_Linux and two bus type declarations for X_1553 and ARINC_629. The processor type declaration for Intel_Linex includes a requires bus access declaration for the bus X_1553. HS_1553 and the bus type declaration X_1553 includes a requires bus access for the bus ARINC_629.HS_629. These required accesses are shown in the graphic on the right side of Table 6-5. The implementation declarations for both buses are also shown in the textual specification in Table 6-5.

Table 6-5: A Sample Bus Specification: Textual and Graphical Representation

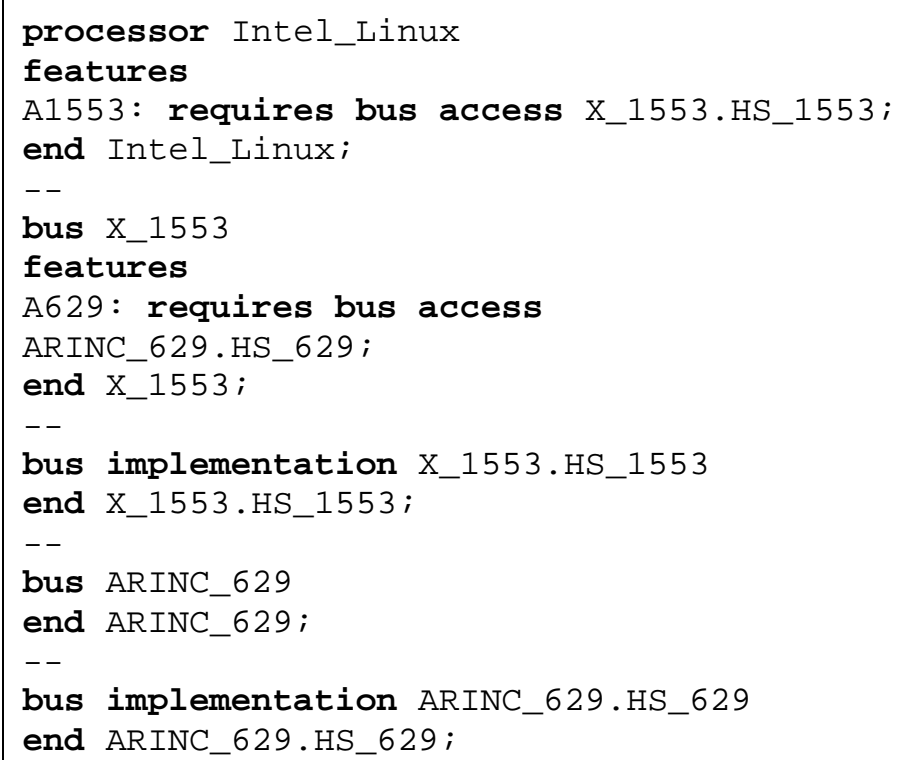

\subsubsection{Properties}

There are a number of predeclared properties that can be used to specify important bus characteristics:

- transmission characteristics such as allowed connection and access protocols, message sizes, transmission time, propagation delay

- hardware source language descriptions

- data movement time characteristics such as the time to move a byte or block of data and any fixed data movement overhead time 


\subsubsection{Constraints}

Table 6-6 summarizes the permitted elements of bus type and implementation declarations.

Table 6-6: Summary of Permitted Bus Declaration Subclauses

\begin{tabular}{|c|l|l|}
\hline Category & \multicolumn{1}{|c|}{ Type } & \multicolumn{1}{|c|}{ Implementation } \\
\hline \multirow{5}{*}{ bus } & $\begin{array}{l}\text { Features } \\
\text { - requires bus access }\end{array}$ & $\begin{array}{l}\text { Subcomponents: } \\
\text { Flow specifications: no }\end{array}$ \\
& Properties yes & Subprogram calls: no \\
& & Connections: no \\
& & Flows: no \\
& & Modes: yes \\
& & Properties yes \\
\hline
\end{tabular}

A bus component can only be a subcomponent of a system component. A summary of the allowed subcomponent relationships and features is included on pages 117-119 in the Appendix.

\subsection{Device}

Device abstractions represent entities that interface with the external environment of an application system. Those devices often have complex behaviors. They may have internal processors, memory, and software that are not explicitly modeled. Alternatively, they may require driver software that is executed on an external processor. A device's external driver software may be considered part of a processor's execution overhead, or it may be treated as an explicitly declared thread with its own execution properties. Examples of devices are sensors and actuators or standalone systems such as a Global Positioning System.

\subsubsection{Textual and Graphical Representations}

A device can interact in complex ways with other components. For example, a device may have a physical connection to a processor via a bus as well as logical connections through ports to application software components. As with all logical connections among components residing on distinct execution platform elements, these logical connections must be supported by (be bound to) physical connections.

Table 6-7 shows an excerpt from an AADL specification that describes a device Roll_Rate_Sensor interacting through a bus with a processor Intel_RTOS. The processor executes the device driver for the Roll_Rate_Sensor. The requirement for bus access is specified in the type declaration for Roll_Rate_Sensor. Similarly, the need for bus access is declared within the processor type declaration for Intel_RTOS. Notice that the out data port declared on the roll rate sensor device provides the rate data from the sensor. A device can be used to represent a more complex 
physical element, such as an engine where the ports can represent the engine's sensors and actuators.

Table 6-7: A Sample Device Specification: Textual and Graphical Representation

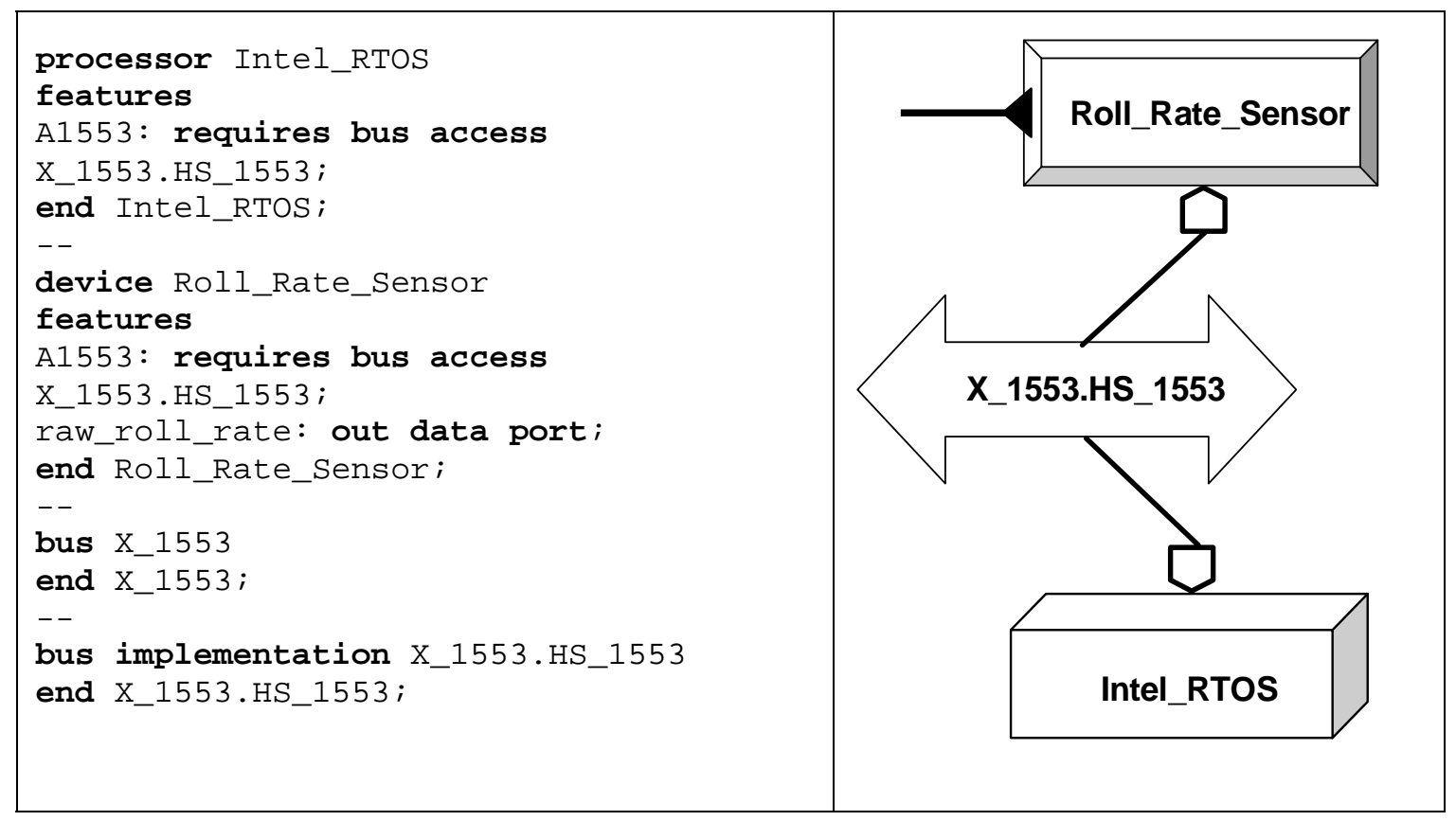

Devices can be viewed from different perspectives. They are integral to the execution environment, both in terms of the application computing system (software and execution platform components) and the physical environment in which the application system exists. Thus, a device can be viewed as

- a physical component that interfaces with the application software through ports (and port groups), as shown in Figure 6-1

- part of the application system interacting with execution platform components and the application system, as shown in Figure 6-2

- a unit in the environment that is accessed or controlled by the application system, as shown in Figure 6-3

The complexity and nature of interactions of a device depend upon how it is included in the architecture. If a device is included as part of the execution platform system, there are numerous logical connections to the application system. If it is included as part of the application system, there are physical connections via bus access across the system hierarchy. In general, it is preferable to place the device declaration with the application code, since the emphasis is on its interaction with the application and the number of connections to the execution platform is then limited to the bus. 


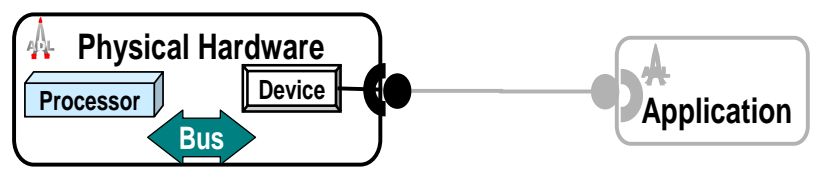

Figure 6-1: $\quad$ A Device as Part of the Physical Hardware

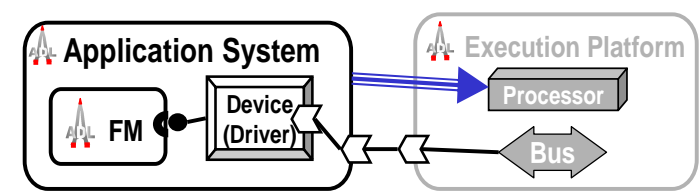

Figure 6-2: $\quad$ A Device as Part of the Application System

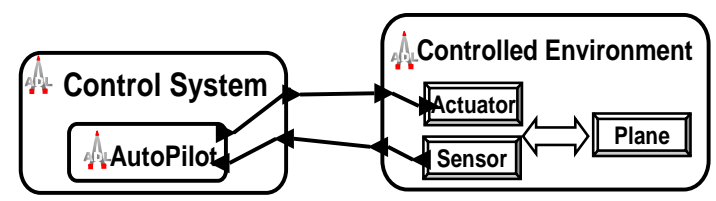

Figure 6-3: $\quad$ A Device as Part of the Controlled Environment

The data port, port group, and connections abstractions - along with their graphical representations - depicted in Figure 6-1 through Figure 6-3 are discussed in Section 8: Component Interactions.

\subsubsection{Properties}

Device properties encompass the dual software and hardware character of a device.

- software-specific properties

- source code files

- source code language

- code size

- execution platform binding properties

- execution platform (hardware) properties, such as those specifying the files that contain the hardware description language for the device and the language used for that description

- properties for specification of the thread properties of the device software executing on a processor, such as dispatch protocols and execution time-related properties 


\subsubsection{Constraints}

Table 6-8 summarizes the permitted elements of device type and implementation declarations. A device component can only be a subcomponent of a system component. A summary of the allowed subcomponent relationships and features is included on pages 117119 in the Appendix.

Table 6-8: Summary of Permitted Device Declaration Subclauses

\begin{tabular}{|l|l|l|}
\hline Category & \multicolumn{1}{|c|}{ Type } & \multicolumn{1}{|c|}{ Implementation } \\
\hline & Features & Subcomponents: \\
& $\bullet \quad$ port & none \\
device & $\bullet \quad$ port group & Subprogram calls: no \\
& $\bullet \quad$ server subprogram & Connections: no \\
& Flows: yes \\
& Flow specifications: yes & Modes: yes \\
& Properties yes & Properties: yes \\
\hline
\end{tabular}




\section{System Structure and Instantiation}

This section presents the language abstractions for structuring and integrating AADL elements into a complete representation of an application system that includes a system component, component bindings, source code elements, and instantiation.

\subsection{System Abstraction}

The system abstraction represents a composite of software, execution platform, or system components. System abstractions can be organized into a hierarchy that can represent complex systems of systems as well as the integrated software and hardware of a dedicated application system (e.g., flight navigation system or database server). Used early in the modeling process to generically represent a component, system components can be formed into a model that is transformed later-some system components being translated into process components and contained components being translated into thread and thread group components.

\subsubsection{Textual and Graphical Representations}

A system can consist of various combinations of software, execution platform, and system components. For example, a system may consist only of software (i.e., process or data components) or execution platform components. Thread and thread group components cannot be subcomponents of a system, since they must be contained within a process or a thread group.

The composition of a system implementation is declared through subcomponent declarations. Table 7-1 provides textual and graphical representations of a system implementation of the system type integrated_control. The details of the type declaration are not included. The explicit subcomponent declarations are shown in the system implementation declaration of integrated_control.integrated_control_system. However, many of the other subclauses are omitted. The supporting declarations are not shown (e.g., the process type declaration for the process type controller). In the graphical portrayal of the system implementation, the subcomponents of integrated_control_system of the type integrated_control are shown. 
Table 7-1: A Sample System Specification: Textual and Graphical Representation
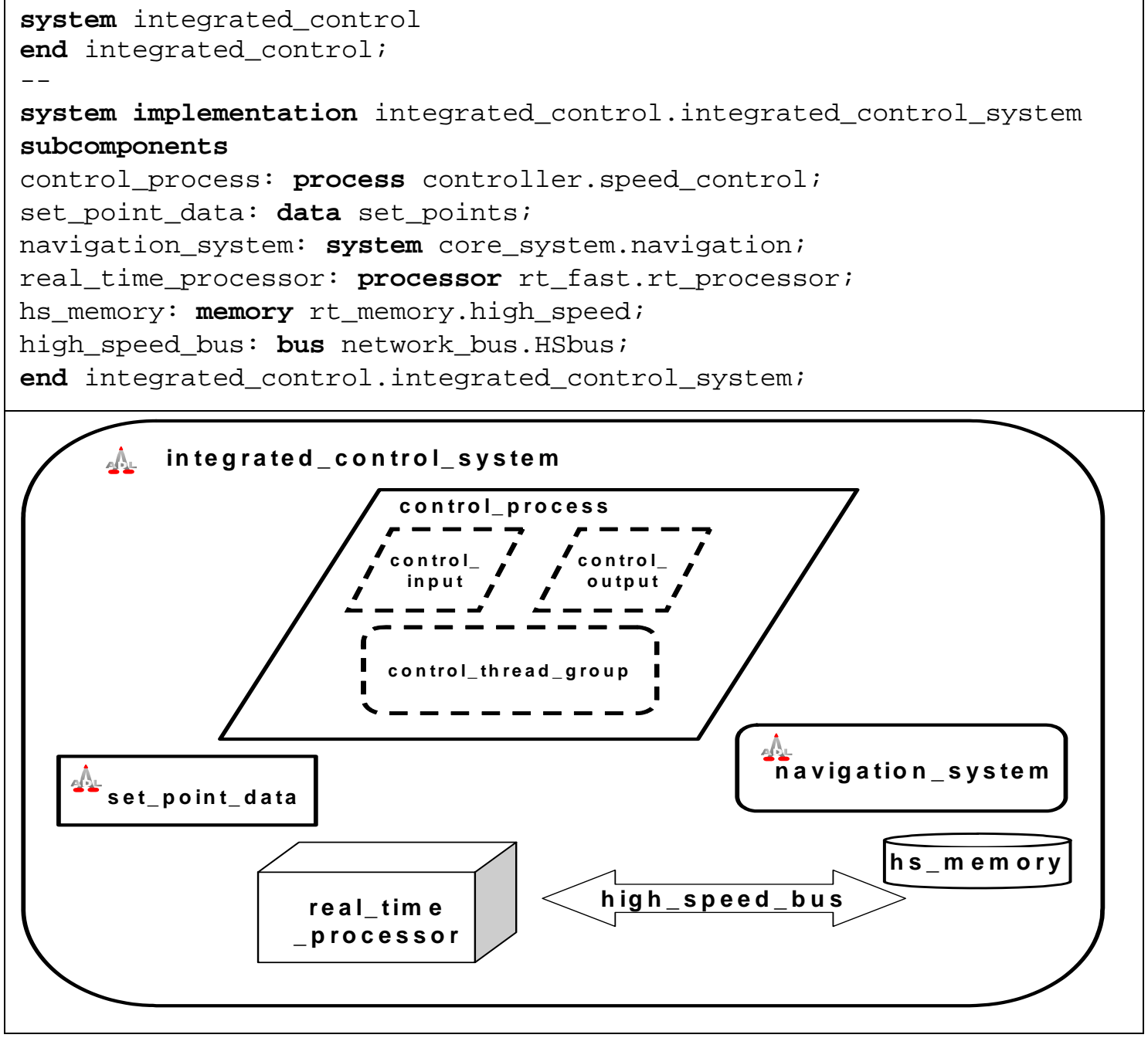


\subsubsection{Constraints}

Table 7-2 summarizes the permitted elements of a system type and implementation declarations. Notice that a system cannot contain a thread or thread group; they must be contained in a process. A system can be a subcomponent only of another system component. A summary of allowed subcomponent relationships and features is included on pages 117-119 in the Appendix.

Table 7-2: Summary of Permitted System Declarations

\begin{tabular}{|c|c|c|}
\hline Category & Type & Implementation \\
\hline system & $\begin{array}{l}\text { Features: } \\
\text { - } \quad \text { server subprogram } \\
\text { - } \quad \text { port } \\
\text { - } \quad \text { port group } \\
\text { - } \quad \text { provides data access } \\
\text { - } \quad \text { provides bus access } \\
\text { - } \quad \text { requires data access } \\
\text { - } \quad \text { requires bus access } \\
\text { Flow specifications: yes } \\
\text { Properties yes }\end{array}$ & $\begin{array}{l}\text { Subcomponents: } \\
\text { - data } \\
\text { - process } \\
\text { - } \text { processor } \\
\text { - memory } \\
\text { - bus } \\
\text { - device } \\
\text { - system } \\
\text { Subprogram calls: no } \\
\text { Connections: yes } \\
\text { Flows: yes } \\
\text { Modes: yes } \\
\text { Properties yes }\end{array}$ \\
\hline
\end{tabular}

\subsection{System Instance}

A system instance represents the runtime architecture of an operational physical system. That physical system may be a stand-alone system or a system of systems. A system instance consists of application software components and execution platform components. Component type and component implementation declarations are architecture blueprints that define the structure and connectivity of a physical system architecture. They must be instantiated to create a complete system instance. A system instance that represents the containment hierarchy of the physical system is created by instantiating a top-level system implementation and then recursively instantiating the subcomponents and their subcomponents.

Once instantiated, the application component instances can be bound to execution platform components (i.e., each thread is bound to a processor; each source text, data component, and port is bound to memory and each connection is bound to a bus if necessary). There is no explicit textual representation for system instances. Instead, system instances are created and stored as system instance models in XML. System instance models can be operated on by analysis and generation tools.

In a fully specified system, the application components are modeled to the level of threads and possibly refined to subprogram calls within threads. Similarly a fully specified execution platform includes processors to execute application code, memory to store 
application code and data, devices that represent the physical environment of the embedded application, and buses that connect these components. Certain system analyses require fully specified system models. For example, scheduling analysis cannot be performed until all the application threads are specified and are bound to processors.

Early in the development process it is desirable to have partially specified system models and be able to instantiate them for analysis. For example, we may represent an application system as a collection of interacting subsystems without providing details of their implementation. Subsystems are modeled as system components or process components. We can instantiate this partial application system together with an execution platform model into a partial system instance model. We can assign resource budgets in terms of CPU cycles and memory requirements to the application subsystems and resource capacities to the execution platform. Given this data we can analyze various bindings of application components to the execution platform and ensure that the budgets do not exceed the capacity. We can also add flow specifications to individual subsystem components and end-to-end flows to the application system. Based on these flow specifications, flow analyses such as an end-to-end response time analysis can be performed without a fully detailed system model. $^{16}$

\subsection{Binding to Execution Platform Components}

For a complete system specification (one that can be instantiated), software components must be bound to appropriate execution platform components. For example, threads must be bound to processing elements and processes must be bound to memory. Similarly, interprocessor connections must be bound to buses, and subprogram calls must be bound to their server subprogram. These bindings are defined through property associations.

There are three categories of binding properties that provide support for declaring:

1. allowed bindings

2. actual bindings

3. identified available memory and processor resources

For example, there is an Allowed_Memory_Binding predeclared property that identifies possible memory components for binding and an Actual_Memory_Binding predeclared property that specifies the memory component to which code and data from source text is bound. The Available_Memory_Binding property specifies the set of contained memory components that are available for the binding to a system's internal components from outside the system. ${ }^{17}$

16 For more information on analysis, see AADL publications and presentations at www.aadl.info.

17 Allowed_Memory_Binding and Actual_Memory_Binding are predeclared properties in the property set AADL_Properties that is part of every AADL specification [SAE 06a]. 


\section{Component Interactions}

Representations of the interactions among components are restricted to defined connections established between interface elements. Connections establish one of the following interactions:

- port connections (Sections 8.1 and 8.2): These are explicit relationships declared between ports or between port groups that enable the directional exchange of data and events among components.

- component access connections (Section 8.3): These are explicit declarations that enable multiple components access to a common data or bus component.

- $\quad$ subprogram calls (Section 8.4): These are explicit declarations within component implementations that enable synchronous call/return access to subprograms.

- parameter connections (Section 8.5): These are relationships among data elements associated with subprogram calls.

Interface elements are declared within the features section of a component type declaration. Paths of interaction (i.e., connections) between interface elements are declared explicitly within component implementations.

\subsection{Ports}

A port represents a communication interface for the directional exchange of data, events, or both (event data) between components. Ports are classified as

- data port: interfaces for typed state data transmission among components without queuing Data ports are represented by typed variables in source text. The structure of the variable/array is defined by the data type [data classifier] on the ports. Connections between data ports are either immediate or delayed.

- event port: interfaces for the communication of events raised by subprograms, threads, processors, or devices that may be queued Examples of event port use include: triggers for the dispatch of an aperiodic thread, initiators of mode switches, and alarm communications. Events such as alarms may be queued at the recipient, and the recipient may process the queue content. Event ports are represented by variables within source text that are associated with runtime service calls.

- event data port: interfaces for message transmission with queuing These interfaces enable the queuing of the data associated with an event. An example 
of event data port use is modeling message communication with queuing of messages at the recipient. Message arrival may cause dispatch of the recipient and allow the recipient to process one or more messages. These ports are represented by port variables in source text that are associated with relevant runtime service calls.

\subsubsection{Port Declarations}

Ports are declared as features in component type declarations. Ports are directional. An out port represents a component's output and an in port represents a component's input. An in out port represents input and output to a component that maps to a single static variable. An in out data port represents both an incoming and an outgoing port such that the outgoing and incoming connections can be made to different components.

The graphical representations for data ports, event ports, and event data ports are summarized in Figure 8-1.

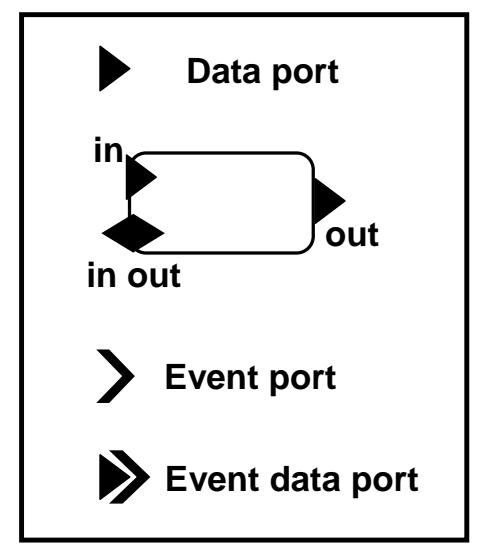

Figure 8-1: Port Graphical Representations

Table 8-1 has an example textual specification and corresponding graphical representation that includes port and port connection declarations. Within component type specifications, appropriate ports declarations are grouped together in the features section. Supporting data type definitions are included at the end of the table. Many of the other details of the specification are not shown. For example, declarations of data types used in data port declarations are not included, as in the declaration of the port c_data_out where the declaration of the data type processed_data is not shown.

In addition to user-defined ports, there are implicitly declared ports for threads. ${ }^{18}$ For example, Error is an implicitly declared out event data port for all threads and may be declared as part of a connection involving a thread. In addition, there is an implicit Complete out event port that, if connected, raises an event, signaling the completion of a thread. Implicit ports can be used directly in connection declarations. They are not included in a features subclause.

18 The predeclared ports for a thread are Dispatch, Complete, and Error [SAE 06a]. 


\subsubsection{Port Connections}

Connection declarations between ports are also shown in Table 8-1. A connection declaration consists of

1. optional identifier (name)

2. $\operatorname{colon}(:)$

3. port connection descriptor (e.g., data port)

4. source port

5. connection symbol (e.g., the symbol $->$ for an immediate connection)

6. destination port

The pattern for port connection textual declaration is shown in the box below:

name: [descriptor] [source port] [connection symbol] [destination port]

Graphically, connections are solid lines between the ports involved in the connection, sometimes with adorned with double cross hatching. See Section 8.1.5 (Immediate and Delayed Communications).

For example, in Table 8-1, the connection c_data_transfer is between the out data port c_data_out of the thread input (written as input.c_data_out) and the in data port c_data_in of the thread control_plus_output (written as control_plus_output.c_data_in). The connections declaration brake_in: event port brake -> input.brake_event; connects the in event port brake of process implementation control.speed_control to the in event port brake_event of the thread subcomponent input. A name for the data port connection between control_plus_output.c_cmd_out and throttle_cmd is not included in this example. The implicit event data port Error is used in the connection error_connection. It is connected to the out event data port Error_Signal but not declared explicitly as a feature in the originating thread.

Table 8-1: Sample Declarations of Data, Event, and Event Data Ports

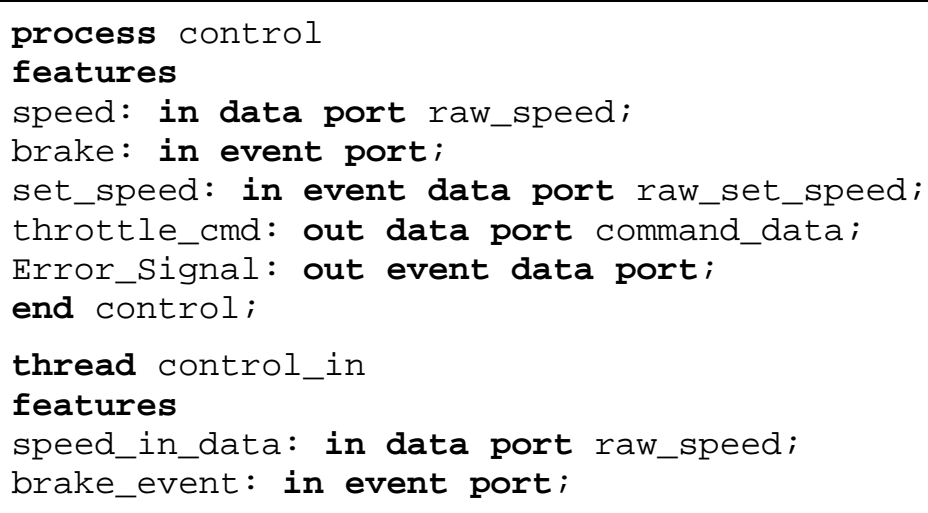


Table 8-1: Sample Declarations of Data, Event, and Event Data Ports (cont.)
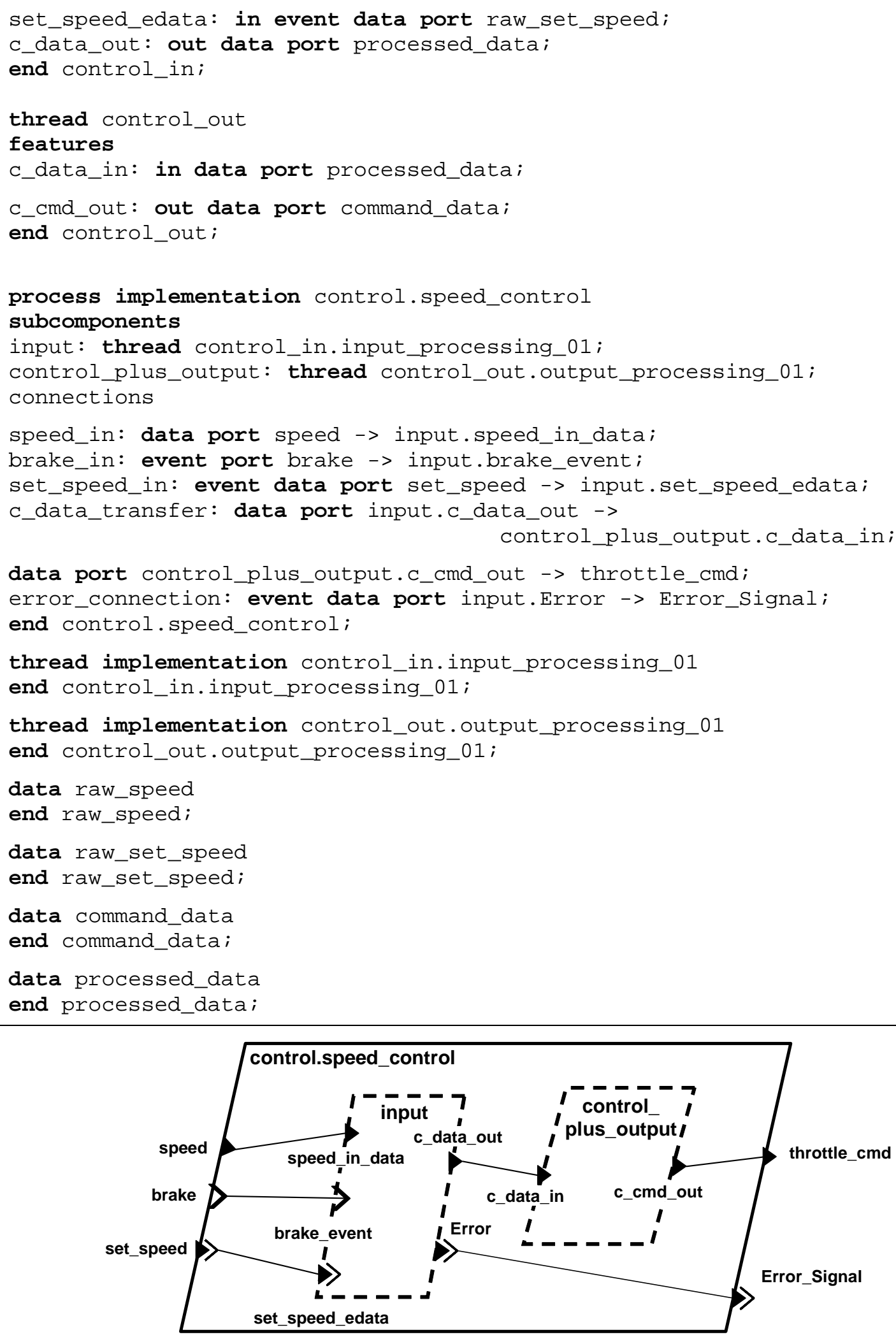


\subsubsection{Connections in System Instance Models}

A connection instance represents the actual flow of data and control between components of a system instance model. In case of a fully specified system, this flow is a transfer between two thread instances, a thread instance and a processor instance, or a thread instance and a device instance. The data flow may be in either direction. However, at least one thread must be included. In the AADL standard, connection instances in a fully specified system model are called semantic connections.

In the case of a partially specified system, the system instance model is expanded through the component hierarchy to the subcomponents for which no implementation detail is provided, regardless of their component category. In this case, connection instances may be between ports of system component instances or process component instances. According to the AADL standard, those connection instances are not semantic connections, but they are essential to certain analyses of partial system instance models.

Connection instances that are semantic connections are illustrated in Figure 8-2. In this figure, data is communicated between two threads in different processes. The data connection between the two threads is expressed by connection declarations that must follow the component hierarchy. In other words, there is a connection declaration from the original thread to its enclosing process, from that process to the second process, and from that process to the contained destination thread. Note that threads cannot arbitrarily communicate with other threads in the system. The enclosing process determines, through the ports in its type declaration and the connection declarations to those ports, which data from its threads should be passed on to threads in other processes.

In a system instance model, the sequence of data connection declarations from a thread to its enclosing process, to the second process, and to the thread contained in the second process results in a connection instance. If two threads are subcomponents within the same process or thread group, the connection instance is represented by a single connection declaration between those threads in the enclosing component

implementation. While there may be a series of port-to-port connections involved in a data transfer (system instance connection) between two threads, data is transferred directly from the sending thread to the receiving thread. From an application source code perspective, the sending thread assigns a value to a variable/array and the receiving thread receives that value in a corresponding variable/array. 


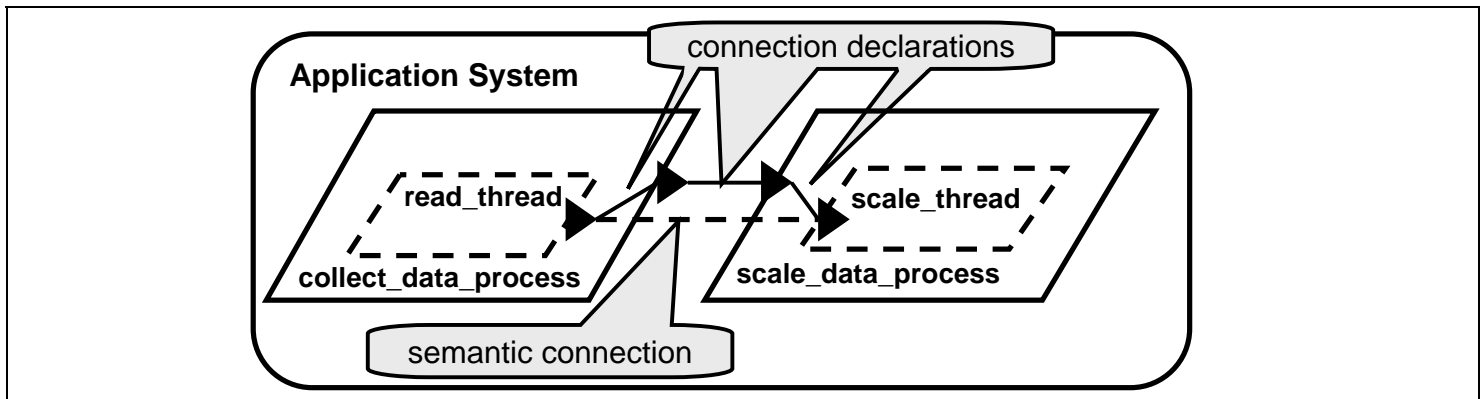

Figure 8-2: $\quad$ A Semantic Connection between Thread Instances

Figure 8-3 illustrates a connection instance in a partial system instance model. In this model, the data collection process and the data scaling process have not been detailed out. The data connection between the two processes results in a connection instance in the system instance model. This connection instance is not considered a semantic connection according to the AADL standard, but the connection instance can be used in a fault propagation analysis or flow analysis of this partially specified system.

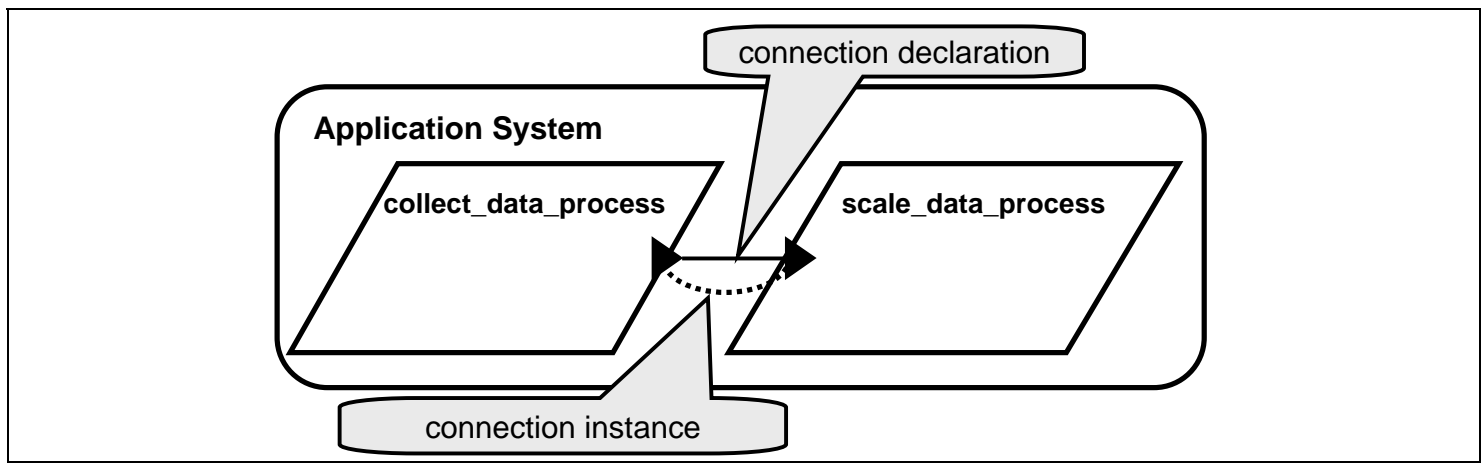

Figure 8-3: $\quad$ A Connection Instance in a Partially Specified System Instance Model

\subsubsection{Port Communication Timing}

The timing of system instance data communication via ports depends upon the type of components involved (i.e., thread, device, or processor) and the nature of their connections. Communication timing is expressed in terms of execution completion, deadline, and dispatch times. For data port transfer out of threads, the data is ready for transfer at the completion of the thread, regardless of dispatch or scheduling characteristics. The timing of the delivery of the data to a receiving component is established by the nature of the data connection between them-immediate or delayed.

For event and event data ports, a source thread executes a Raise_Event call. This call results in the immediate transfer of control for an event port and the immediate transfer of both control and data for an event data port. 


\subsubsection{Immediate and Delayed Communications}

The type of connection between thread data ports establishes specific timing semantics for data that is transferred between originating and terminating threads. Data port connections can be immediate or delayed. This section presents the basic timing semantics for these inter-thread connections. It does not address the potential impact of bus speeds, communication protocols, or partitions on these connections.

For immediate connections, data transmission is initiated when the source thread completes and enters the suspended state. The value delivered to the in data port of a receiving thread is the value produced by the sending thread at its completion. For an immediate connection to occur, the threads must share a common (simultaneous) dispatch. However, the receiving thread's execution is postponed until the sending thread has completed its execution. This aspect can be seen in Figure 8-4, where the immediate connection specifies that the thread control must execute after the thread read_data, within every $50 \mathrm{~ms}$ period. In addition, the value that is received by the thread control is the value output by the most recent execution of the thread read_data.

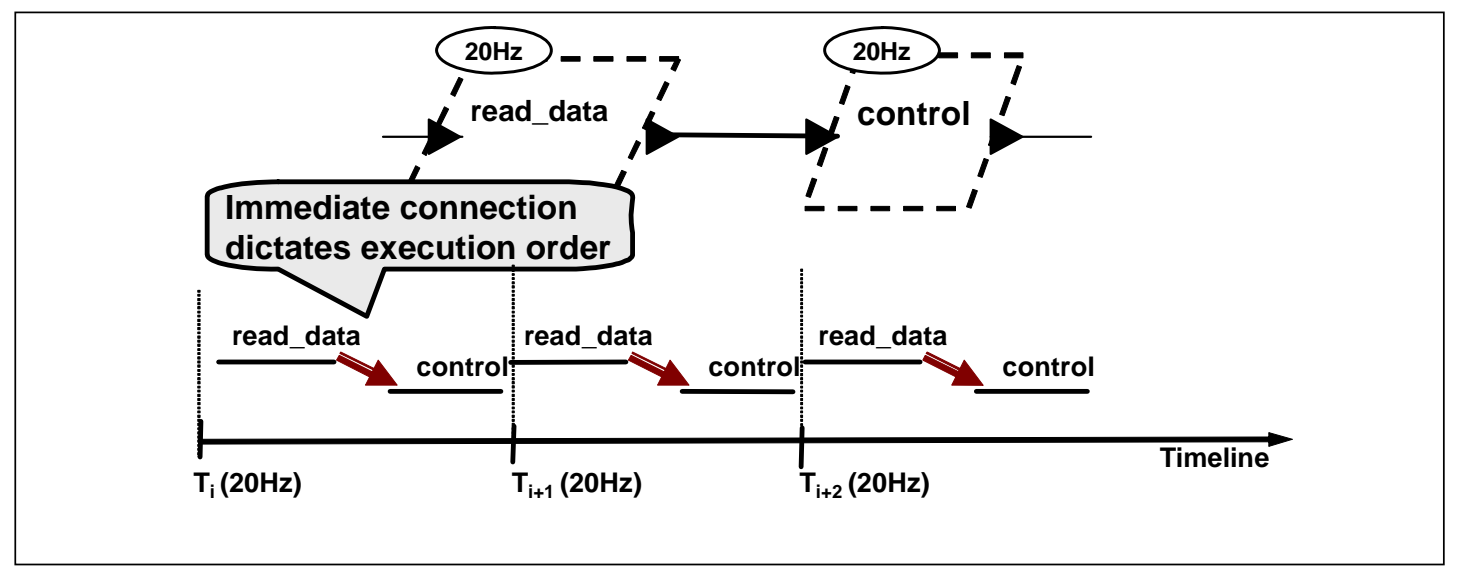

Figure 8-4: $\quad$ An Immediate Connection

For the graphical timelines in Figure 8-4 through Figure 8-9, a horizontal bar above the timeline that is labeled with a thread name represents the execution time of that thread. The left edge represents the start and the right edge represents the termination of the thread's execution. A solid or segmented arrow between thread execution bars represents a data transfer between threads. A segmented arrow represents a delayed (e.g., Figure 8-5) or a repeat transfer (e.g., Figure 8-6).

For the two threads illustrated in Figure 8-4, a partial textual specification is shown in Table 8-2. The connection immediate_C1 is declared as immediate using the single-headed arrow symbol $(->)$ between the out data port and in data port. Notice the Period property association (50 ms) within each of the thread type declarations. 
Table 8-2: AADL Specification of an Immediate Connection

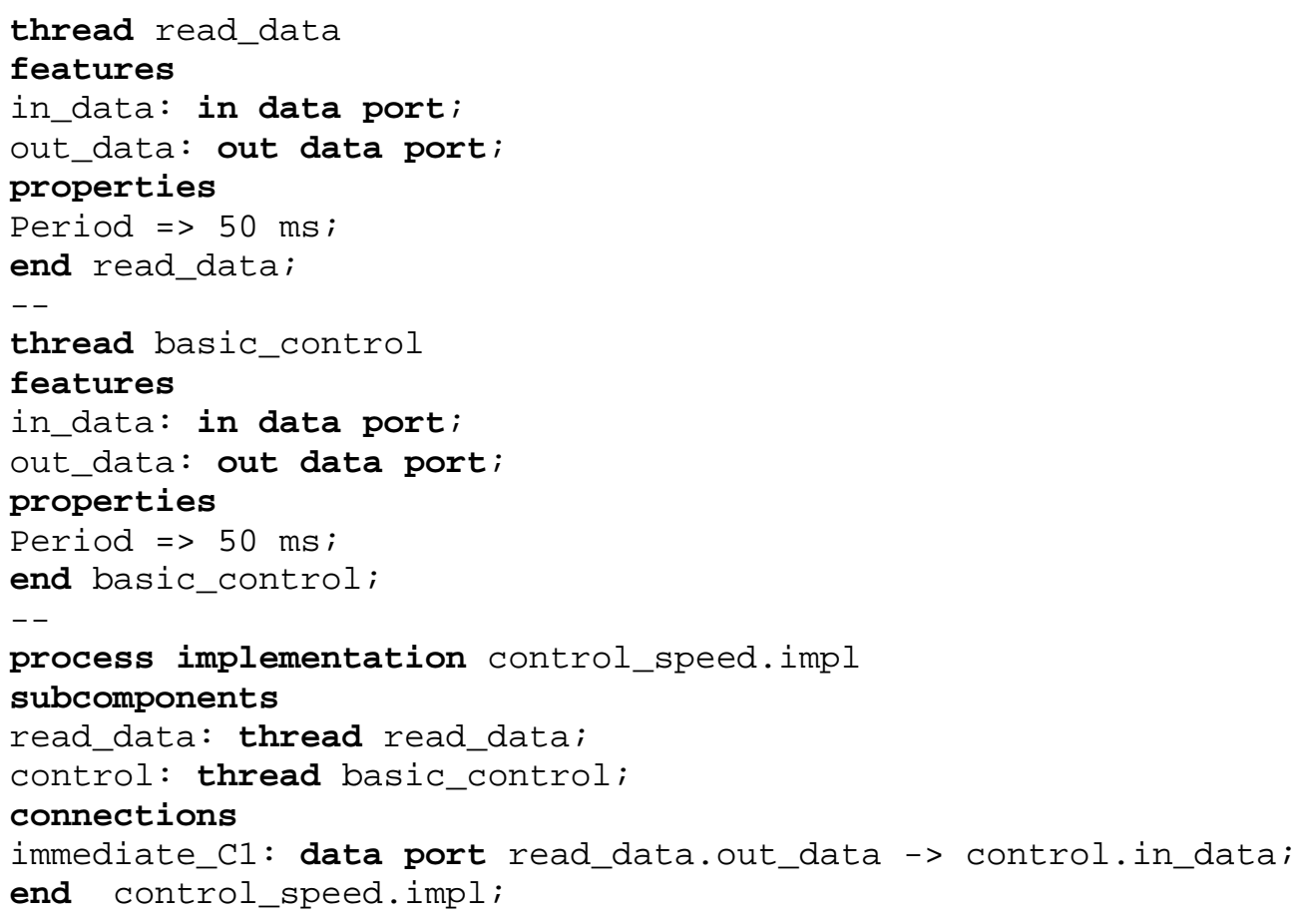

For a delayed port connection, the value from the sending thread is transmitted at its deadline and is available to the receiving thread at its next dispatch. For delayed port connections, the communicating threads do not need to share a common dispatch. In this case, the data available to a receiving thread is that value produced at the most recent deadline of the sending thread. If the deadline of the sending thread and the dispatch of the receiving thread occur simultaneously, the transmission occurs at that instant. The impact of a delayed connection can be seen in Figure 8-5, where the thread control receives the value produced by the thread read_data in the previous $50 \mathrm{~ms}$ frame. A shown in Figure 8-5, a delayed connection is symbolized graphically by double cross hatching on the connection arrow between the ports.

For the two threads illustrated in Figure 8-5, a partial textual specification is shown in Table 8-3. This specification has some differences from the one in Table 8-2: the connection delayed_C1 is declared as delayed using the double-headed arrow (->>) and the Period property association is declared in a properties subclause within the process. This association specifies that the value of $50 \mathrm{~ms}$ is the period of contained threads unless overridden within an individual thread's declaration. 
Section 8: Component Interactions

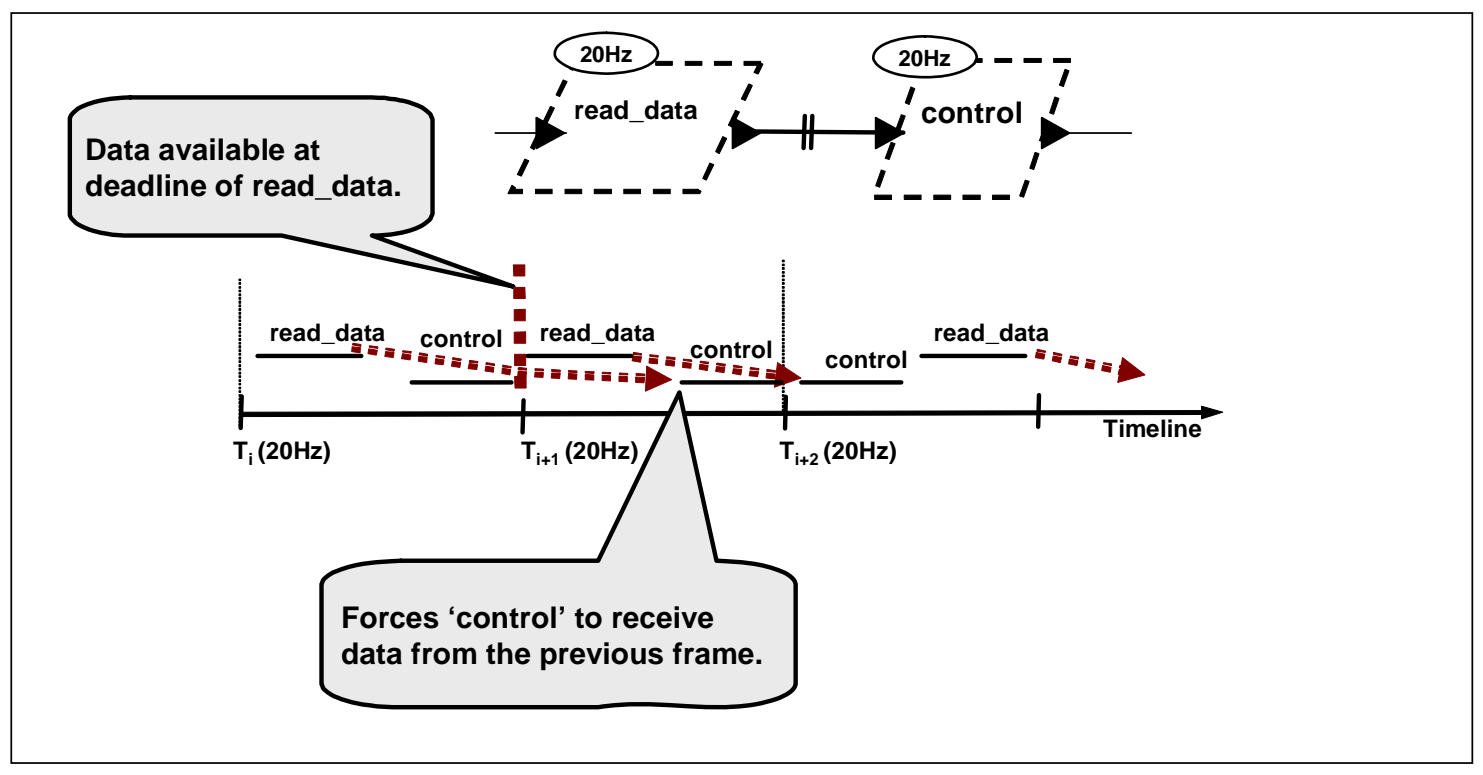

Figure 8-5: A Delayed Connection

Table 8-3: AADL Specification of a Delayed Connection

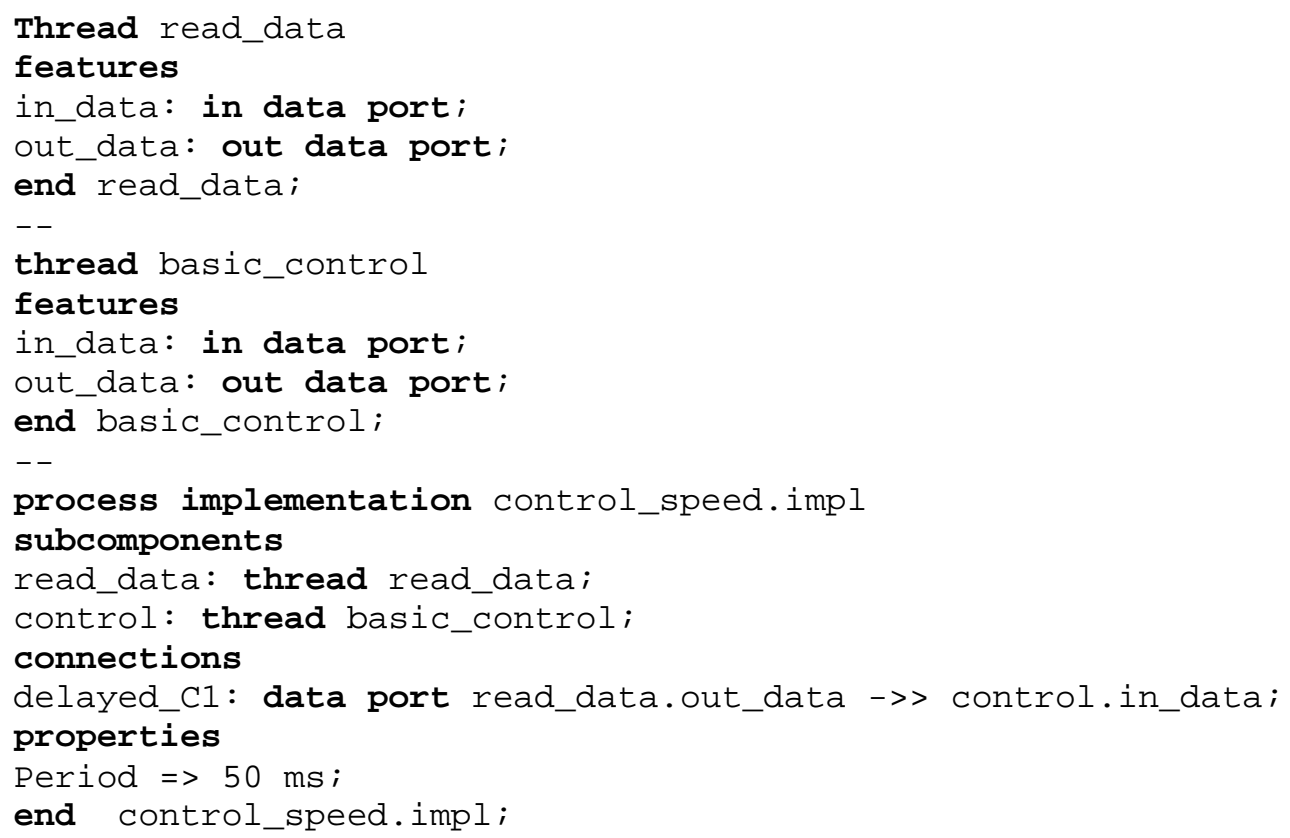

\subsubsection{Oversampling and Under-Sampling}

For communication between different frequency periodic threads with simultaneous dispatch, both delayed and immediate communications can be used to ensure a well-defined exchange.

Consider the example of two simultaneously dispatched threads read_data and control shown in Figure 8-6 and Figure 8-7. In the case of a delayed connection, the value from read_data is available at its deadline. It is received by the two executions of control whose dispatch coincides with or follows that deadline (e.g., read_data may have a 
preperiod deadline). Thus, the two executions of cont rol occurring within an execution frame of read_data receive the value produced in the preceding frame of read_data.

In contrast, consider the case of immediate connections as shown in Figure 8-7, the values available for two sequential executions of control are the same, the value produced within the $10 \mathrm{~Hz}$ execution frame of read_data. This result is accomplished by delaying the execution of the first control within the frame until the completion of read_data. Notice that this can only occur if both read_data and an execution of control can successfully complete (i.e., meet deadline) within the execution frame of control.

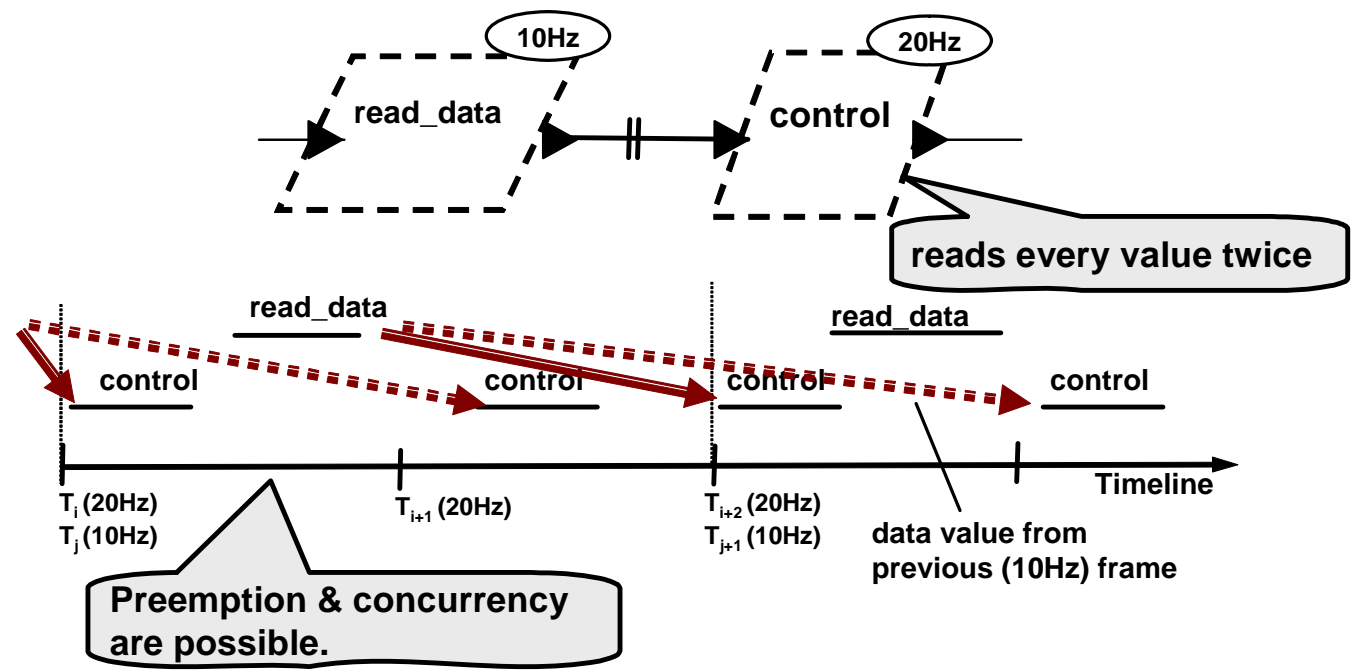

Figure 8-6: $\quad$ Oversampling with Delayed Connections

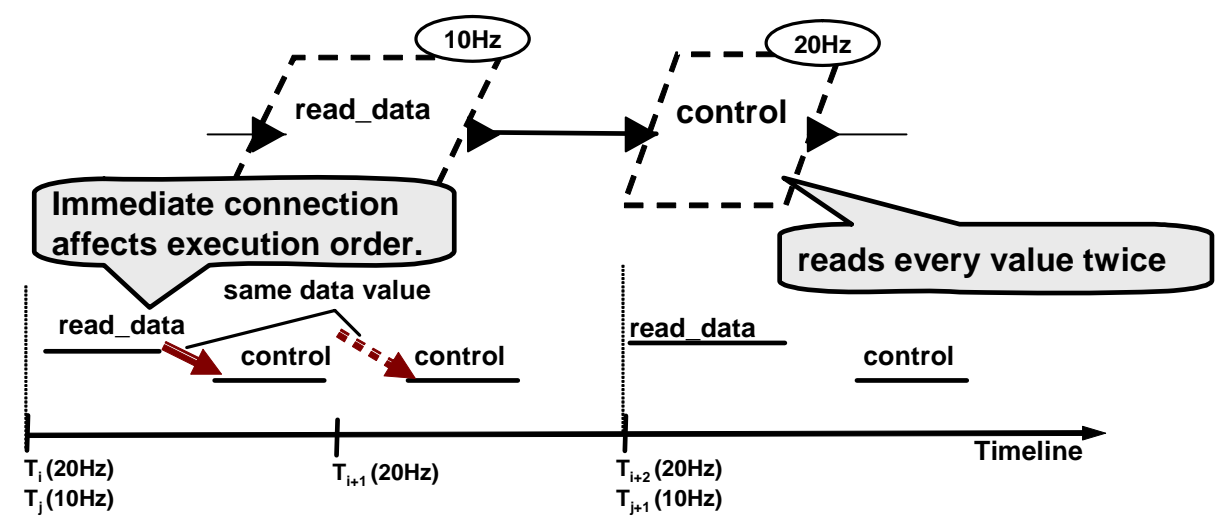

Figure 8-7: $\quad$ Oversampling with Immediate Connections

Consider the situation where a periodic thread is sending to a simultaneously dispatched higher frequency thread. For a delayed connection, as shown in Figure 8-8, the data provided to an execution of control is the value produced by read_data that is available at the simultaneous dispatch of the threads. That value is produced at the most recent read_data deadline, which may coincide with the thread's dispatch. In the case of an immediate connection as shown in Figure 8-9, the value provided to the thread 
control is the value produced by read_data at the end of its first execution after the simultaneous dispatch, and the execution of control is delayed until read_data has completed.
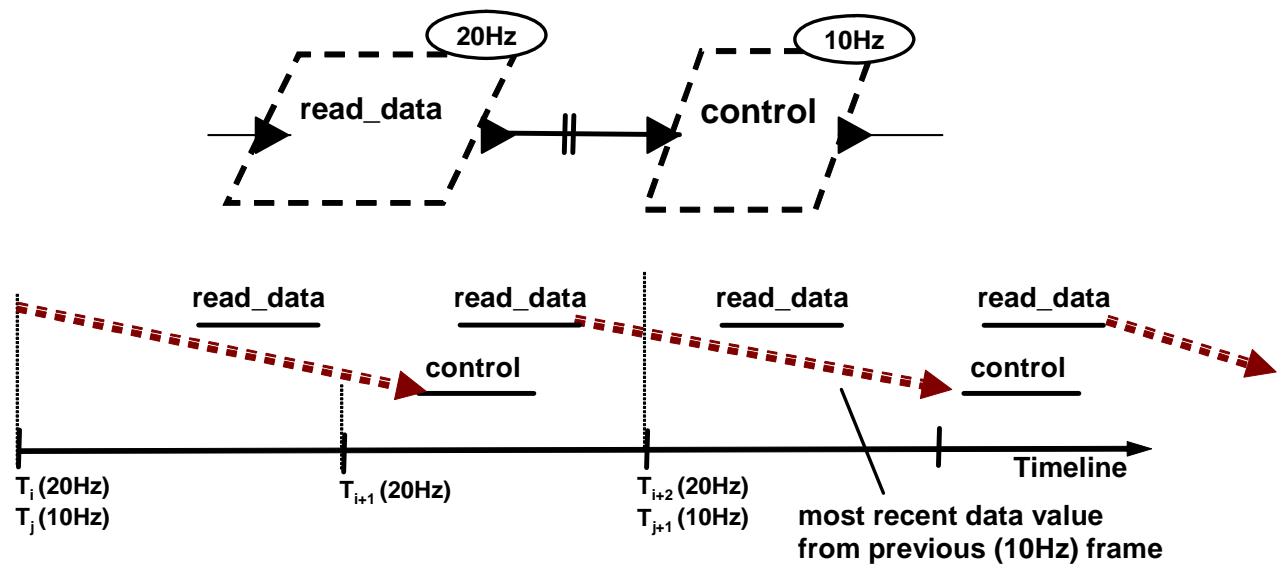

Figure 8-8: $\quad$ Under-Sampling with Delayed Connections
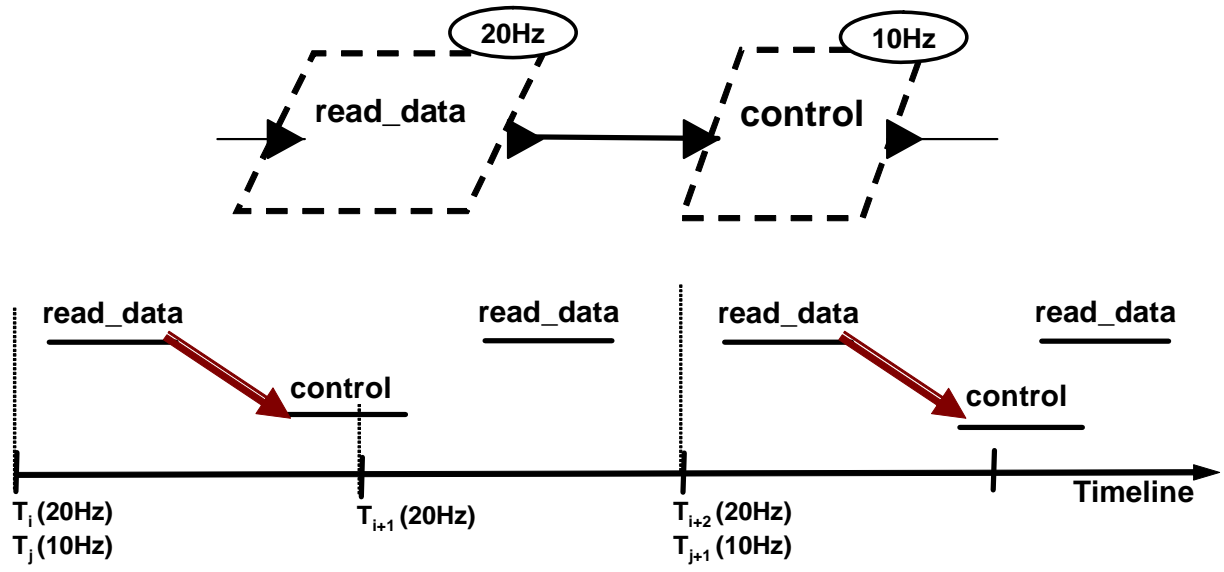

Figure 8-9: $\quad$ Under-Sampling with Immediate Connections

\subsubsection{Properties}

A variety of predeclared port properties provide details on the interface represented by the port, including properties relating to the

- $\quad$ source text for the port

- whether a connection is required for the port

- port binding characteristics

- entry points associated with event and event data ports

For example, Source_Name is used to specify the name of the port variable in the source code. Required_Connection is used to indicate whether the component's implementation is aware of a port's having a connection (i.e., the connection may be 
optional) or whether the component assumes the connection to always be in place. ${ }^{19}$ Portspecific execution time, deadline, and source code entrypoints can be specified for each port to reflect that each may cause a different piece of code to be executed. Several properties allow the queue characteristics of event and event data ports to be specified.

In addition, predeclared port connection properties allow the declaration of specific connection protocols and binding properties relating to the connection. Binding properties allow the declaration of actual and allowed binding as well as the specification of restrictions on the co-location of software elements associated with the connection.

\subsubsection{Port and Port Connection Constraints}

There are restrictions on the topology of port connections. An out data port can be connected to (i.e., send data to) data ports of multiple components - a "fan-out" of data. An in data port, however, is restricted to a single incoming connection. In other words, because it does not support queuing, an in data port cannot have a "fan-in" from different sources; the outputs from those sources would overwrite one another. If queuing of data is desired, an event data port should be used. In contrast, event ports and event data ports support both data fan-out and fan-in. Fan-in is supported because these ports support queuing. Multiple inputs at an event or event data port enable the specification of the sequencing of disparate events as well as the queuing of events.

While it is permissible to omit the explicit declaration of the data type for a data or event data port, the explicit declaration allows checking of consistency of data type and size for the connections made between ports. Thus, the connection from the out data port of the thread read to the in data port of the thread scale in Figure 8-3 requires that the data type declaration for each of these ports and all of the intervening ports must be the same for a complete system specification. However, incomplete port specifications are permitted. For example, it is acceptable for one end of a connection not to have a data type declared while the other end does. Similarly, one end of a connection can have just a data component type while the other end has a data implementation with the same type.

\subsection{Port Groups}

The port group abstraction represents a collection of ports or other port groups. The content and structure of a port group are declared completely through a port group type declaration. There is no implementation declaration. Port groups are declared in the features section of component types and reference a port group type. They may be incompletely specified by not referring to a port group type or by referring to a port group type containing ports that themselves are not completely specified.

19 Source_Name and Required_Connection are in the predeclared property set AADL_Properties that is part of every AADL specification [SAE 06a]. 
Port groups can be used to

- reduce the number of connection declarations

- simplify graphical presentations

- allow a single reference to multiple related ports, connections, and entities in a specification

- group ports with common properties (e.g., all event ports)

- mix port types and directions

\subsubsection{Port Groups and Port Group Type Declarations}

A port group is defined in a type declaration that explicitly identifies the individual ports and port groups that it comprises. Example port group declarations and their declaration as features within a component type are shown in Table 8-4. As with other component type declarations, properties of the port group can be declared and a port group type can be extended and refined.

The declarations in the Table 8-4 are excerpts from a complete specification and include only relevant declarations and portions of declarations needed to show what is required in specifying a specific port group. In the tables, port group type declarations are shown in the left column and example references to the type and supporting declarations are shown in the right column.

Table 8-4: Sample Port Group with Mixed Port Types

\begin{tabular}{|c|c|}
\hline port group type declaration & $\begin{array}{c}\text { port group reference } \\
\text { (with supporting declarations) }\end{array}$ \\
\hline $\begin{array}{l}\text { port group roll_set } \\
\text { features } \\
\text { roll_data: in data port; } \\
\text { roll_cmd: out data port c_form; } \\
\text { engage: in event port; } \\
\text { errors: port group error_set; } \\
\text { end roll_set; } \\
\text { data c_form } \\
\text { end c_form; } \\
\text { port group error_set } \\
\text { features } \\
\text { sensor_error: in data port; } \\
\text { range_error: out event port; } \\
\text { end error_set }\end{array}$ & $\begin{array}{l}\text { process control } \\
\text { features } \\
\text { roll_o1: port group roll_set; } \\
\text { end control; }\end{array}$ \\
\hline
\end{tabular}

A port group type can be declared as the inverse of another port group type. This relationship is indicated by the reserved words inverse of and the name of a port group type. The features of the inverted port group must be in the same order as in 
the referenced port group but with the opposite directions. A port group type that is named in an inverse of statement cannot itself contain an inverse of statement.

Thus, a chaining of inverses, such as $B$ inverse of $A$ and $C$ inverse of $B$, is not permitted. An example of the use of the key word inverse of is shown in Table 8-5.

Table 8-5: A Port Group Type Declaration and its Inverse

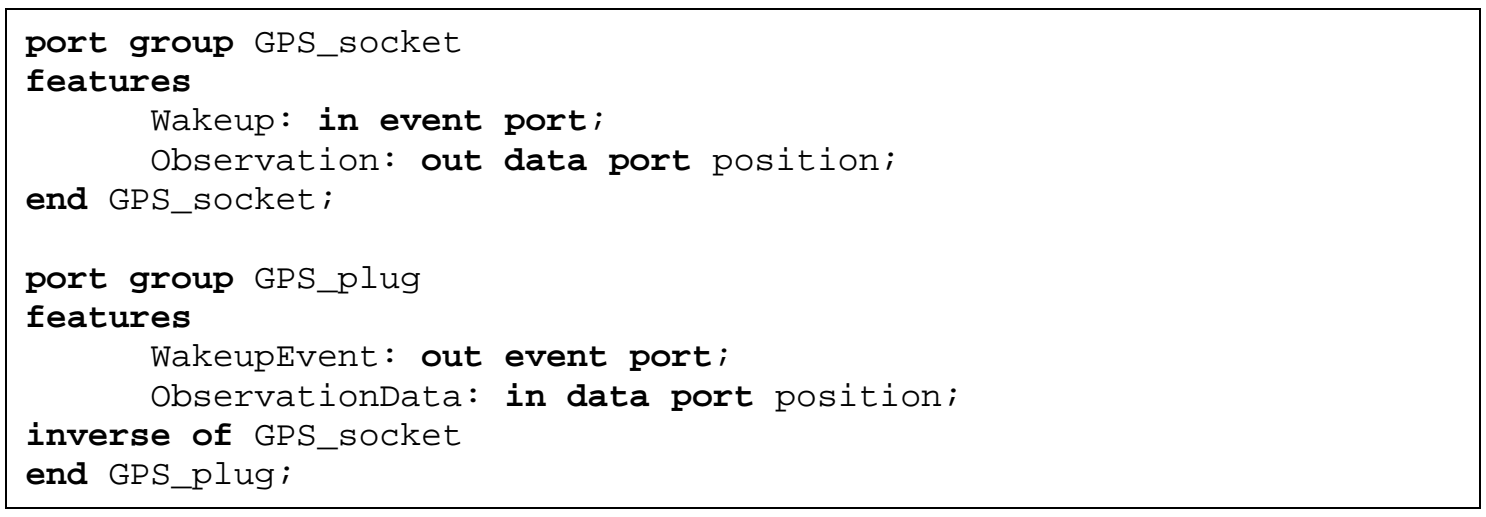

Figure 8-10 contains graphical icons for port groups and their connections. The graphical symbols of a port group represent the features declaration of the port group within a component type declaration. Port groups can bundle different port types and directions.

\begin{tabular}{|l|l|}
\hline \multicolumn{1}{c}{ Port Group } \\
(as a feature of a thread) \\
\hline $\begin{array}{l}\text { Port Group Connection } \\
\text { (between two port groups that } \\
\text { are each a feature of system) }\end{array}$ \\
\hline $\begin{array}{l}\text { Port Group Bundle } \\
\text { (mixed directions and ports) }\end{array}$
\end{tabular}

Figure 8-10: Graphical Representations of Port Groups

\subsubsection{Port Group Connections}

Connections can be made between port groups, individual ports, and the individual ports within a port group. Within a component, elements of a port group in its component type can be individually connected to ports of subcomponents. However, elements of a port group of a subcomponent cannot be individually connected to other subcomponents. In other words, grouping and pulling apart elements of a port 
group can occur when going up or down the component hierarchy, but not within the same level of the component hierarchy.

Figure 8-11 shows a graphical representation of a port group identified as mode_control_group and its inverse, with relevant excerpts from a corresponding AADL specification for a simple cruise control system. The connection declaration between the port groups is shown in Table 8-6 that includes excerpts from an AADL specification.

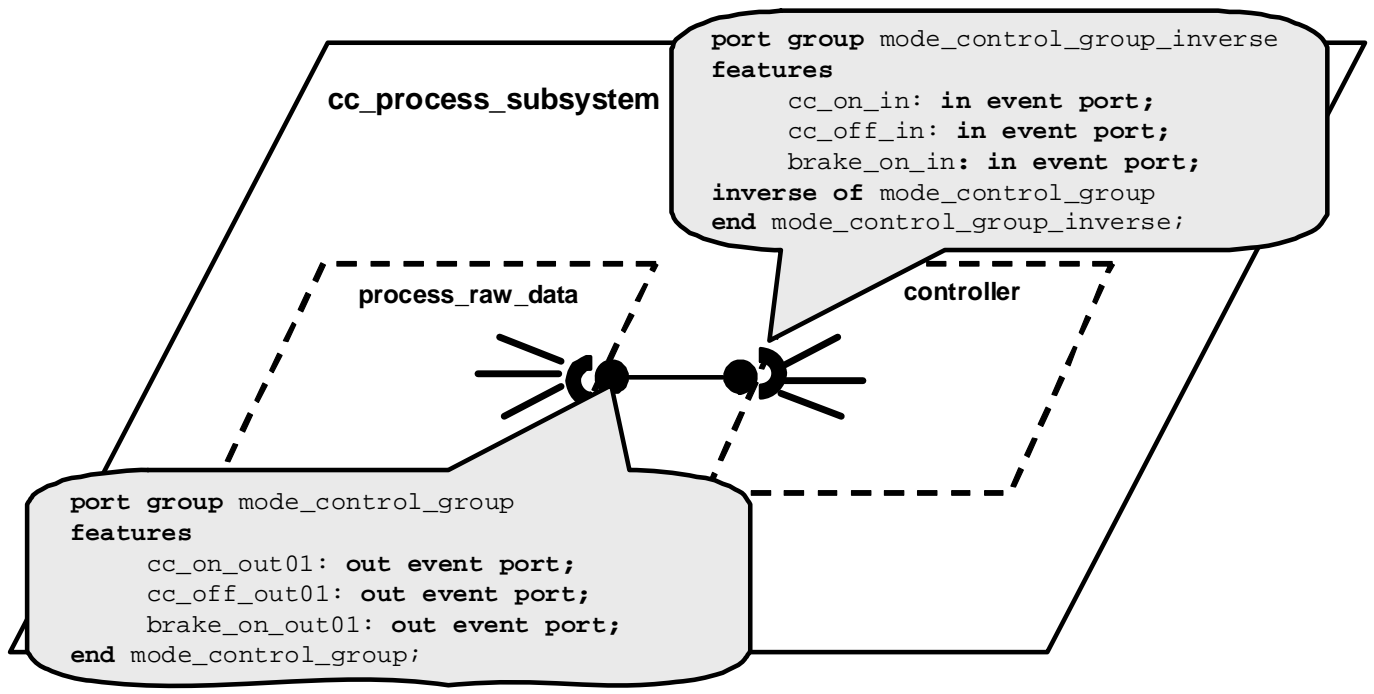

Figure 8-11: Sample Port Group Connections

Table 8-6: Sample Port Group Connection Declarations

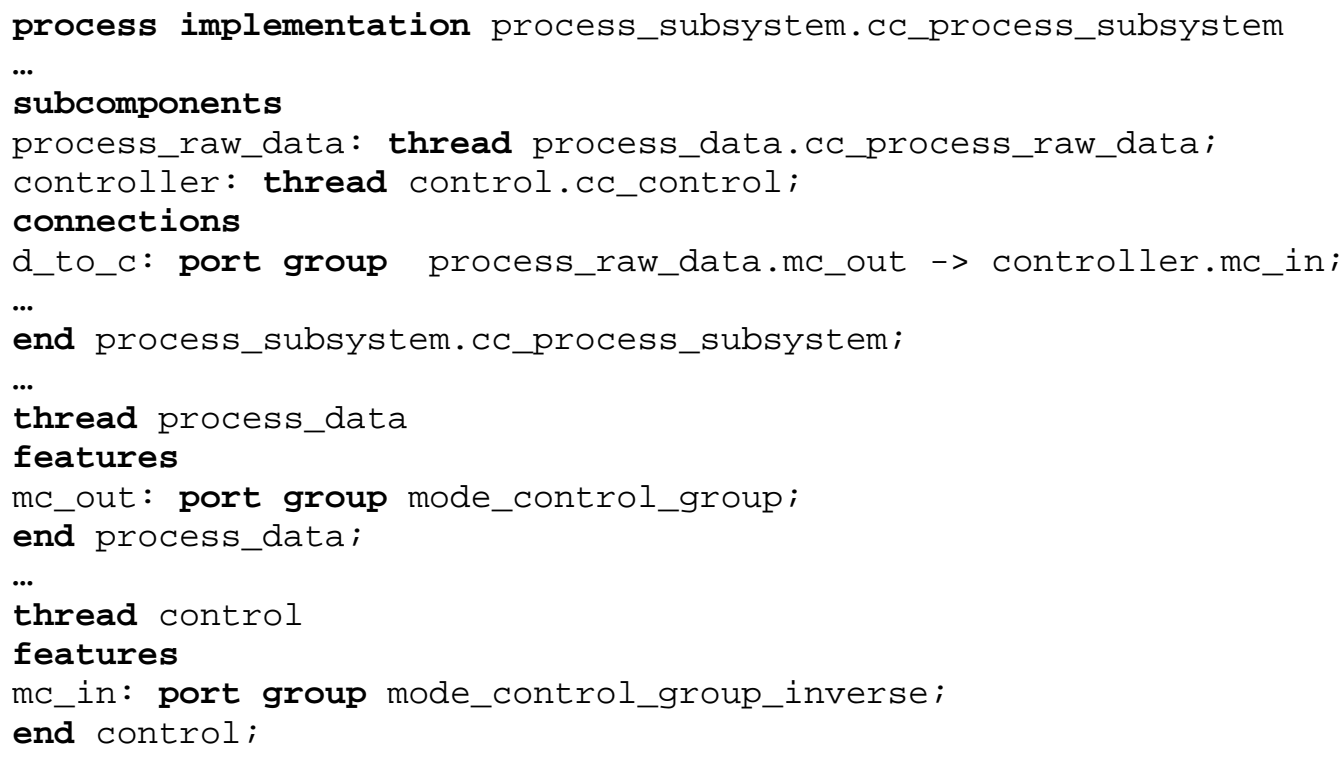

Port groups can be effective in grouping related data and connections. For example, the individual outputs of multiple sensors (devices) within a sensor subsystem (grouped in a system) can be bundled together into a single port group. In that instance, all of the 
sensor data is transferred through a single connection declaration from the sensor subgroup to a control processing system. The information provided by the ports within the port group is distributed through separate connections to individual control processing subsystems.

\subsubsection{Aggregate Data Ports}

Time consistency in data transmission can be achieved using an aggregate data port group. An aggregate data port group consists exclusively of data ports that have the same direction (i.e., all out data ports) with an Aggregate_Data_Port property value of true. ${ }^{20}$ For this specialized port group, data transmission from multiple ports is time coordinated - that is, if data associated with the port group is produced by a set of simultaneously dispatched periodic threads, the recipients of that data receive a consistent set of values from the most recent dispatch or a consistent set of values from the previous dispatch of the threads.

\subsubsection{Properties}

Predeclared port group properties can be used to establish a port group as an aggregate data port and define port group memory binding characteristics. Port group connections can have properties that reflect the properties of the ports that compose the port group. For example, there is a Source_Text property that specifies the source files associated with the port group and an Allowed_Memory_Binding property that specifies the set of memory components to which data and event data ports within the port group can be bound.

\subsection{Subcomponent Access}

Data and bus subcomponents are made accessible throughout a system through explicit features declarations within type declarations of components. For data components, this capability supports modeling of shared access to a common data area or static data. For bus components, this access models the connectivity of execution platform components through buses whose access they share.

The access declarations are

- provides: indicates that a component provides access to a data or bus component contained within it

- requires: indicates that a component requires access to a data or bus component that is external to it

20 Aggregate_Data_Port is a predeclared property for every AADL specification [SAE 06a]. 


\subsubsection{Data Access Declarations}

Examples of a data subcomponent access declaration are shown in Table 8-7. There is an optional identifier for the declaration.

Table 8-7: Data Access Declarations

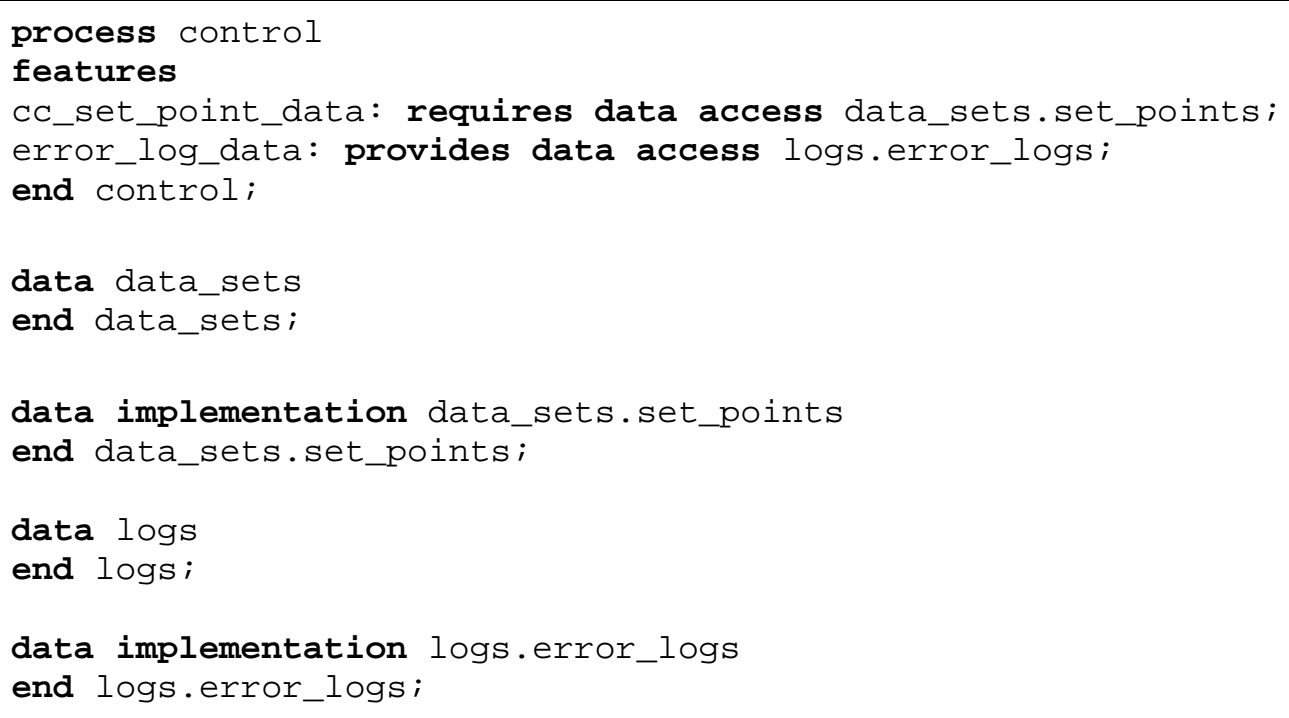

\subsubsection{Data Access Connections}

The connections (paths) for subcomponent access are declared in connections declarations within component implementations. The access connection specifies the path from the component providing access to the component requiring access (i.e., from provides to requires).

Table 8-8 presents an example of data access connections declarations. The lower portion of Table 8-8 is a graphical representation of these data access dependencies. The example shows some of the declarations for the system implementation basic_control.auto_cc that are relevant to the data access relationships for the system. The thread subcomponent cc_algorithm of the process cc_control requires access to the local data subcomponent comm_error_log (logs.error_logs). In addition, the thread subcomponent comm_errors requires access to the data subcomponent comm_error_log (logs.error_logs) of the process cc_error_monitor. This connection is a remote connection across address spaces, where the process cc_control provides access to its data subcomponent.

Notice the concurrent access to the data subcomponent comm_error_log (logs.error_logs) in the example. The predeclared property Concurrency_Control_Protocol can be used to coordinate this access (e.g., to ensure mutually exclusive access). Other predeclared properties for data subcomponent access identify whether the required or provided access is read_only, write_only, or 
read_write.A Required_Access property association must be the same as the Provided_Access property of the component that is accessed. ${ }^{21}$

Table 8-8: Shared Access across a System Hierarchy

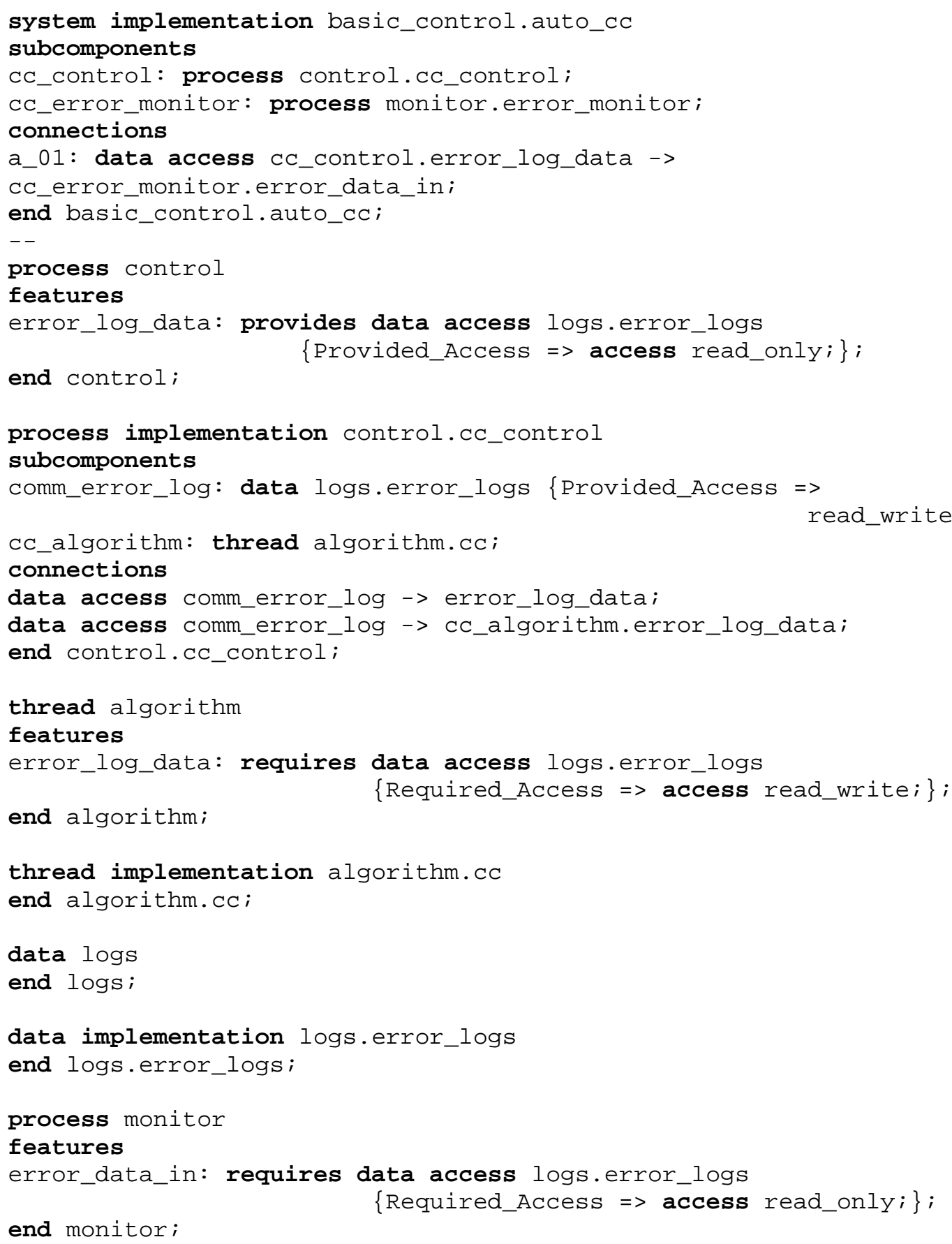

21 The predeclared properties Concurrency_Control_Protocol, Required_Access, and Provided_Access are included in the property set AADL_Properties. This property set declaration is part of every AADL specification [SAE 06a]. 
Table 8-8: Shared Access across a System Hierarchy (cont.)
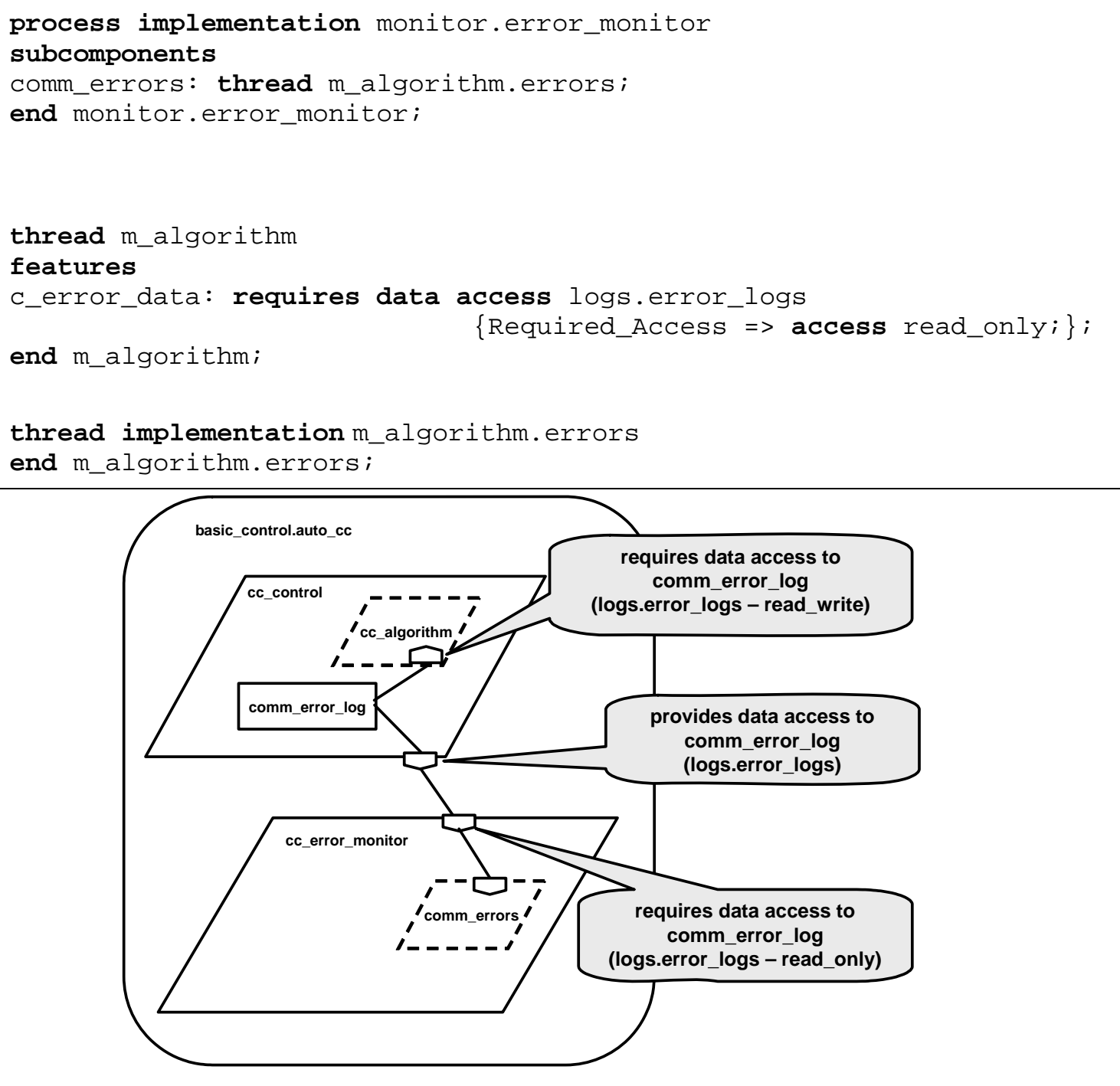

\subsubsection{Bus Access and Bus Access Connections}

In addition to access to data, access to buses is declared explicitly in AADL. Table 8-9 shows an example of bus access for a simplified cruise control system that consists of a cruise control unit (system component) and driver input, speed sensor, and throttle devices. The additional execution hardware for the system consists of a processor that executes the cruise control system application software and a bus connecting the hardware components. The figure in the lower portion of Table 8-9 is a graphical representation for required access features and connections to the bus declared in the text. It also shows the data connections for the system. Some of the details of the subcomponent declarations are not complete in the sample specifications. 
Table 8-9: Basic Bus Access and Access Connection Declarations

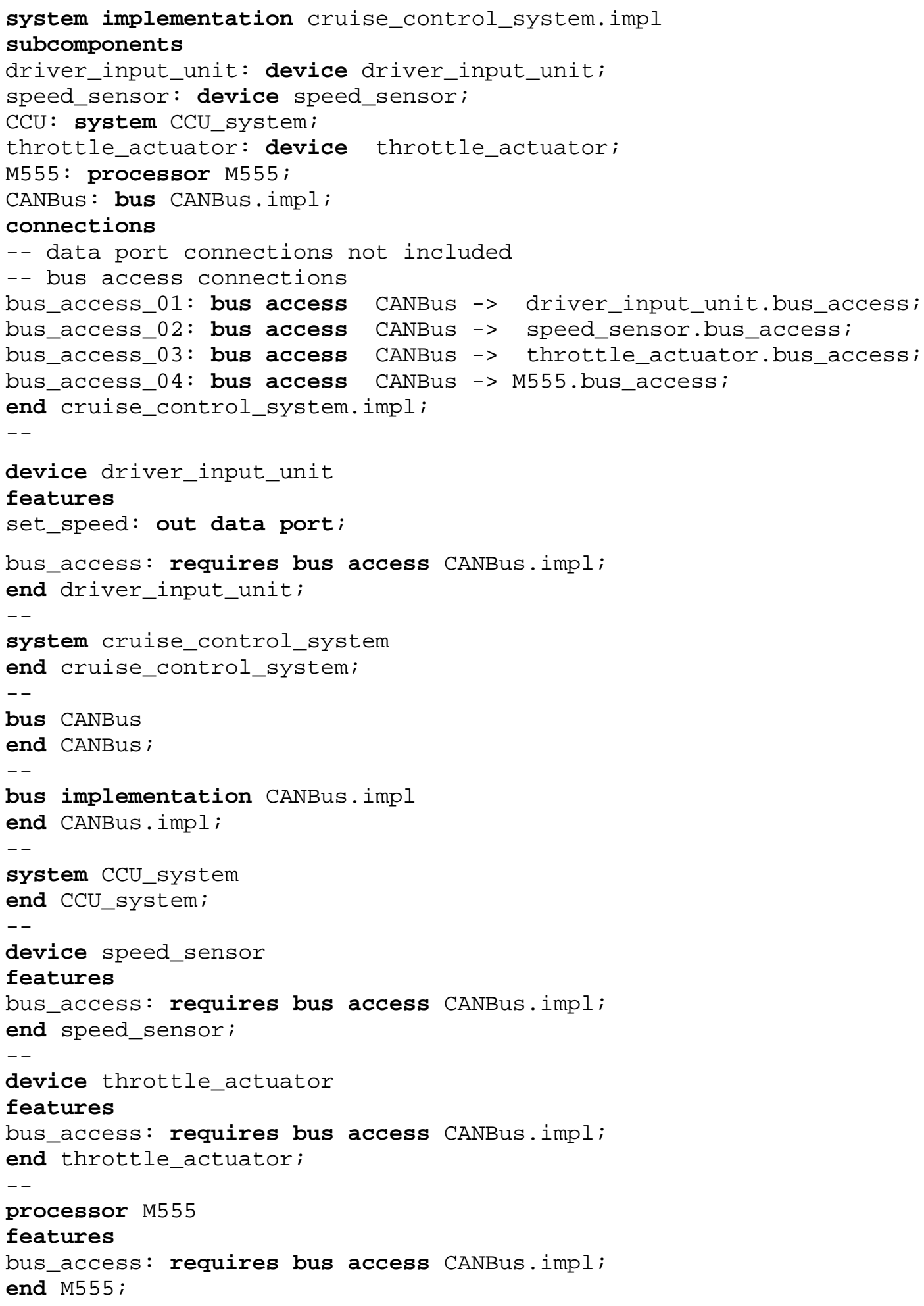


Table 8-9: Basic Bus Access and Access Connection Declarations (cont.)

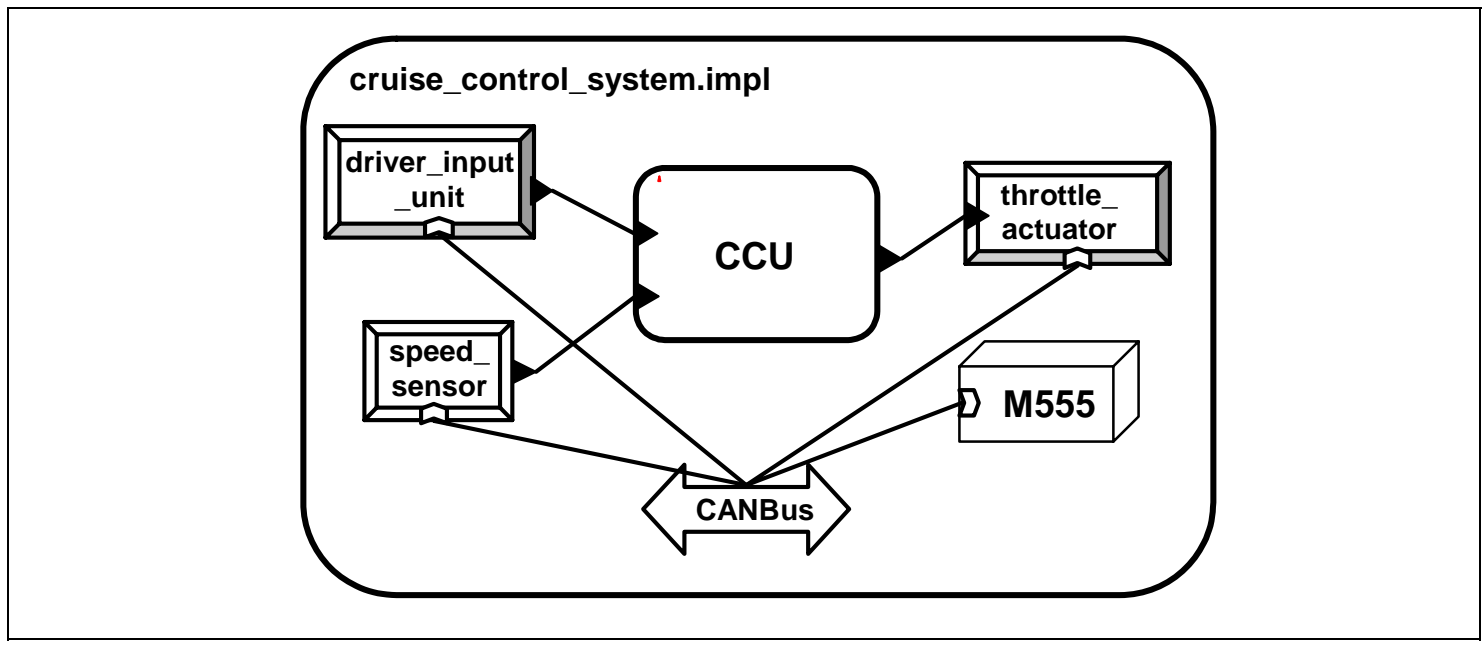

Table 8-10 illustrates how to model two subsystems with hardware components and bus connections. Some of the specifications are not complete (e.g., type rather than implementation classifiers are used in defining some of the components and subcomponents). In the illustration, one subsystem is connected to the other by a bus provided by the second subsystem. Specifically, the application system requires bus access to the network system's 1553 bus. The bus access, requires, provides, and connections are shown both graphically (lower portion of Table 8-10) and as AADL text declarations.

\section{Table 8-10: $\quad$ Example Bus Access Connection Declarations}

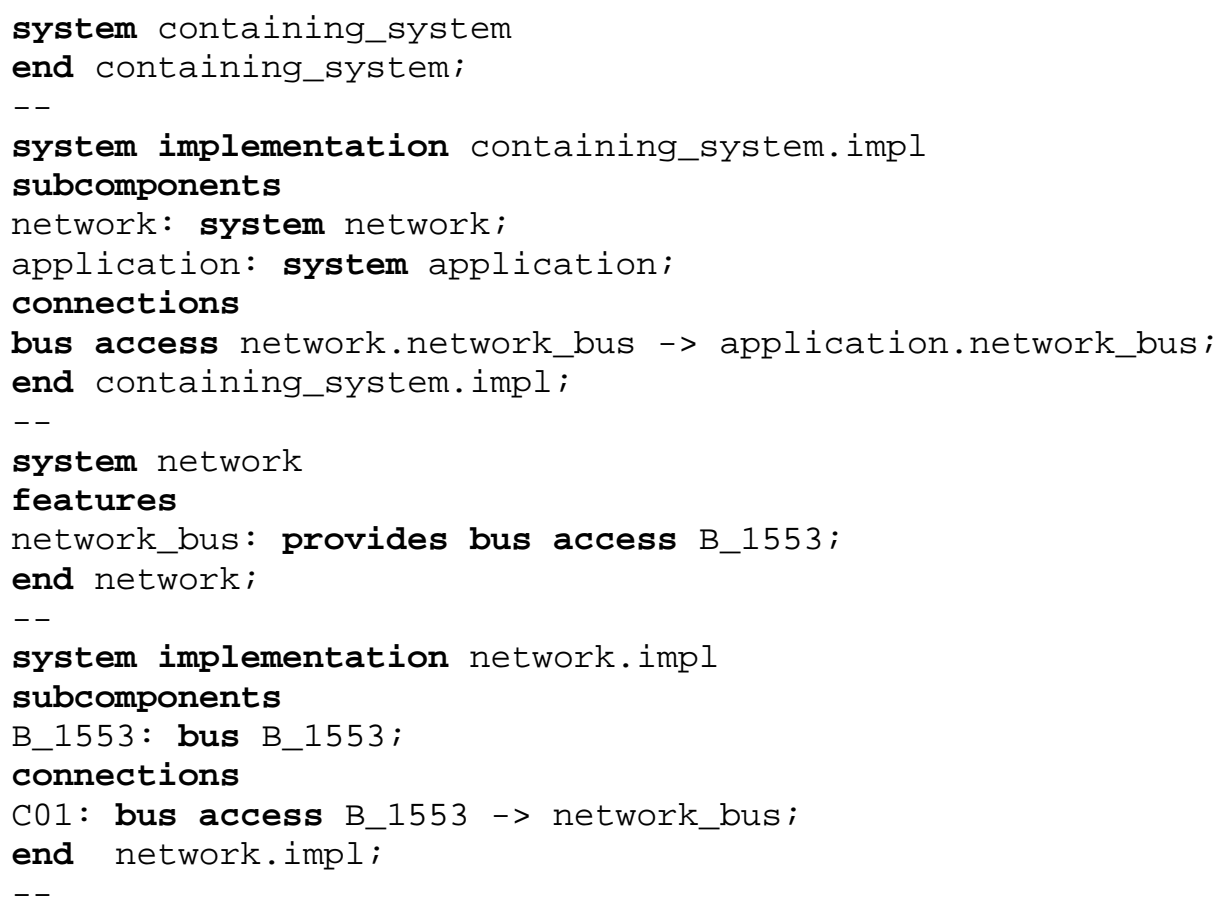


Table 8-10: Example Bus Access Connection Declarations (cont.)
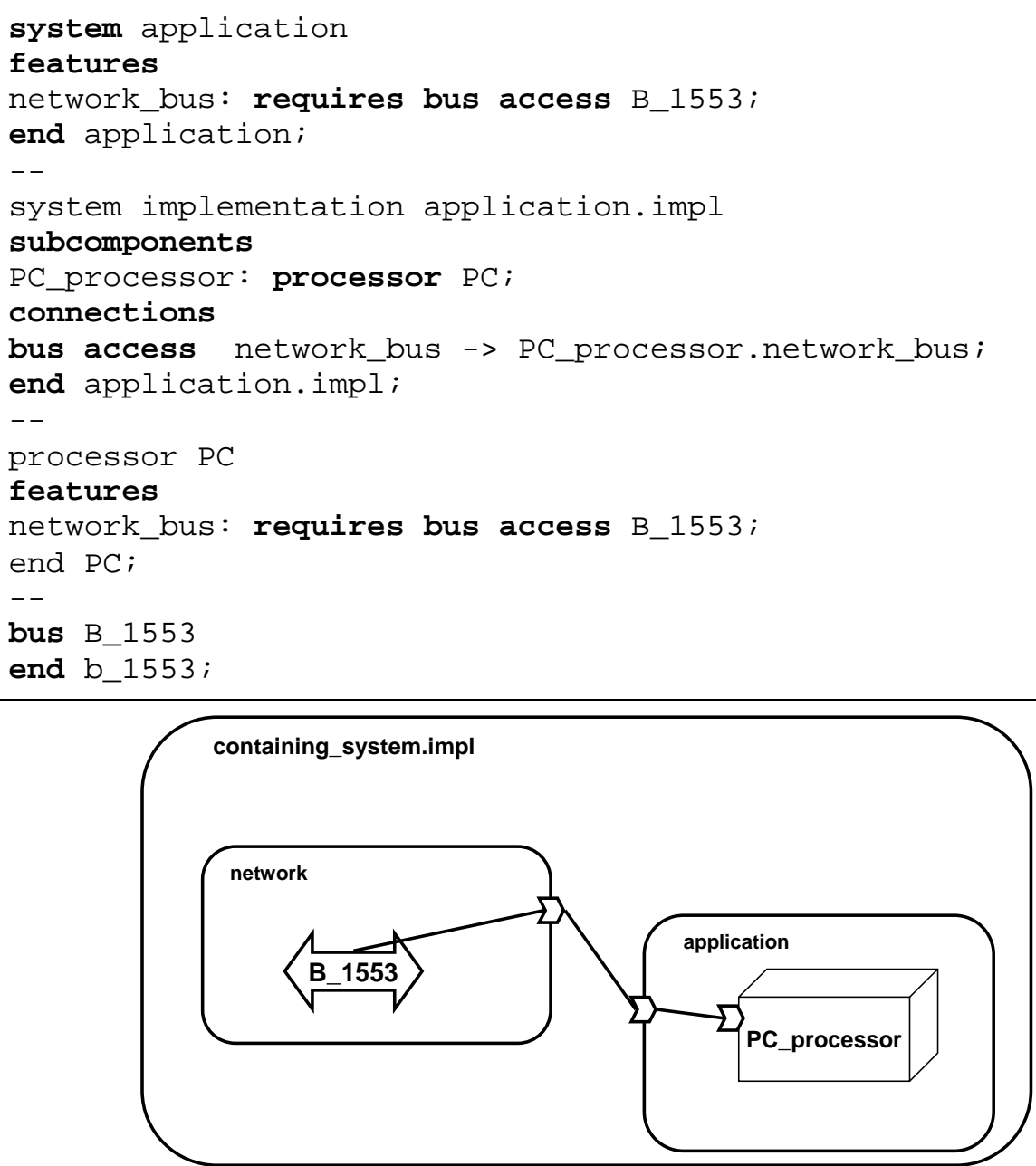

\subsection{Subprogram Calls}

Subprogram calls are declared through calls declarations within a thread or subprogram implementation. The subprogram that is called must be declared through a subprogram type declaration and possibly a subprogram implementation declaration, as discussed in the Section 5.5.1 (Subprogram Declarations).

In the current version of the AADL standard, subprograms are not declared as instances through a subprogram subcomponent declaration. The need for such instances is inferred from the calls and can take into account sharing of subprogram libraries across processes. The specific subprogram called is declared through a property association of the predeclared property Actual_Subprogram_Call. The example in Table 8-12 illustrates this principle. 


\subsubsection{Call Sequences}

There may be a sequence of calls declared within a component implementation. An example is shown in the partial specification of Table 8-11 where the calls sequence two_calls involves a call to the subprogram implementations acquire . temp and then adjust. level. The associated subprogram declarations are also shown. The calls sequence is determined by the subprogram calls declaration order. In other words, the calls order is linear. If more complex call orderings are desired, an annex notation could provide specification of other orderings, such as a "branch" or "iteration." Alternatively, one can specify different calls sequences that are active under different modes. For more details on the use of modes, see Section 9 (Modes).

Notice that subprograms may call other subprograms. This circumstance is shown in Table 8-11 where the subprogram implementation adjust. level calls the subprogram find.temp_values.

Graphically, subprogram calls are represented by subprogram symbols, arranged left to right within a thread implementation or subprogram symbol. A call sequence arrow may be included as shown in the figure in the lower potion of Table 8-11.

\section{Table 8-11: Example Subprogram Calls}

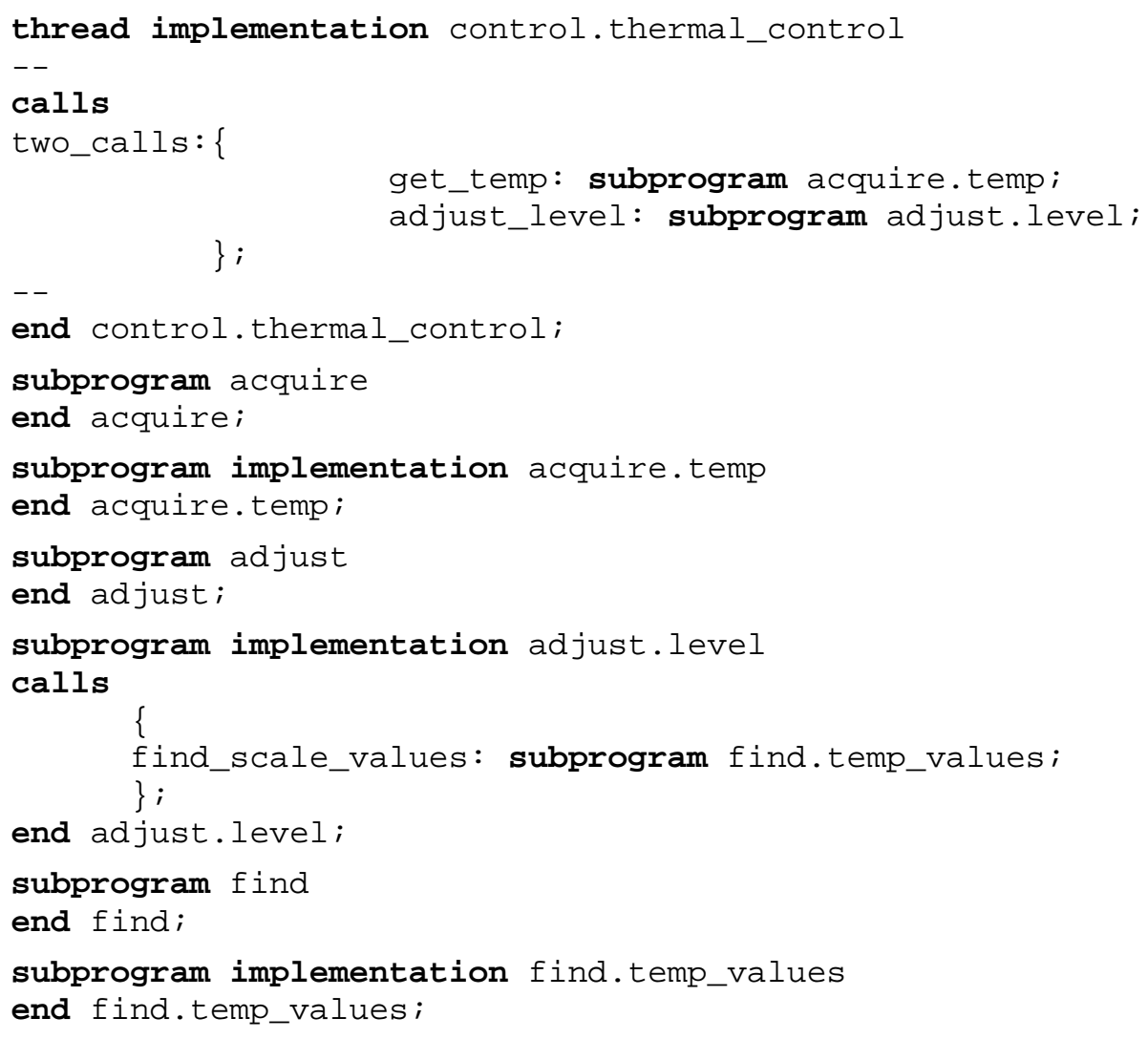


Table 8-11: $\quad$ Example Subprogram Calls (cont.)

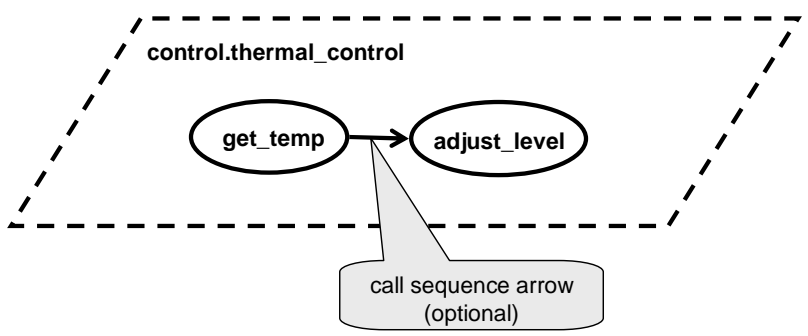

\subsubsection{Remote Calls}

Remote client-server interactions can be modeled using server subprogram calls as shown in the partial specification in Table 8-12. The property association Actual_Subprogram_Calldeclares that the subprogram call call_server within the thread calling_thread, which is a subcomponent of the process client_process, is being made to the subprogram contained within the server process (server_process). This is an example of a contained property association that is discussed in more detail in Section 11.2.2 (Contained Property Associations).

Table 8-12: Client-Server Subprogram Example

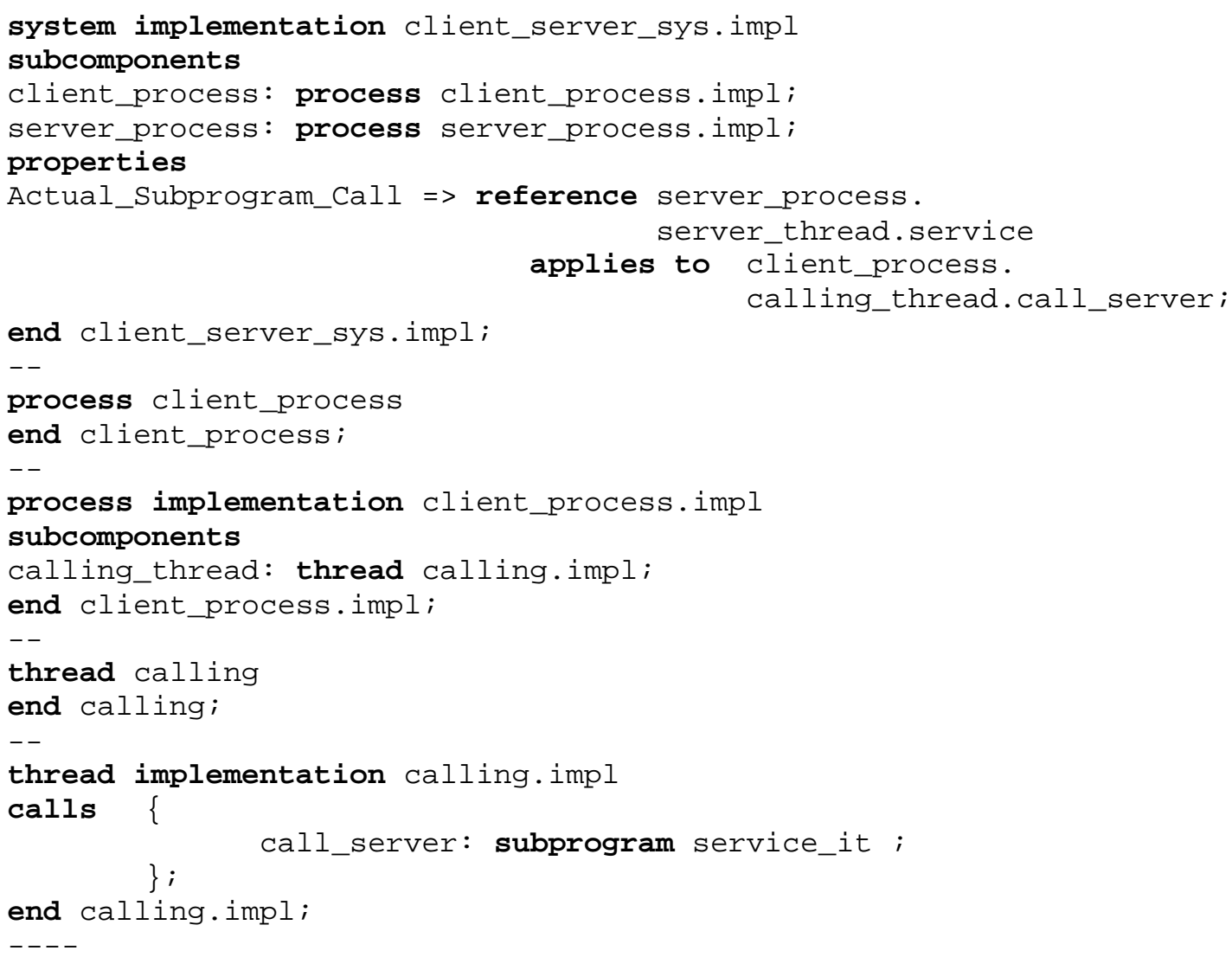


Table 8-12: $\quad$ Client-Server Subprogram Example (cont.)
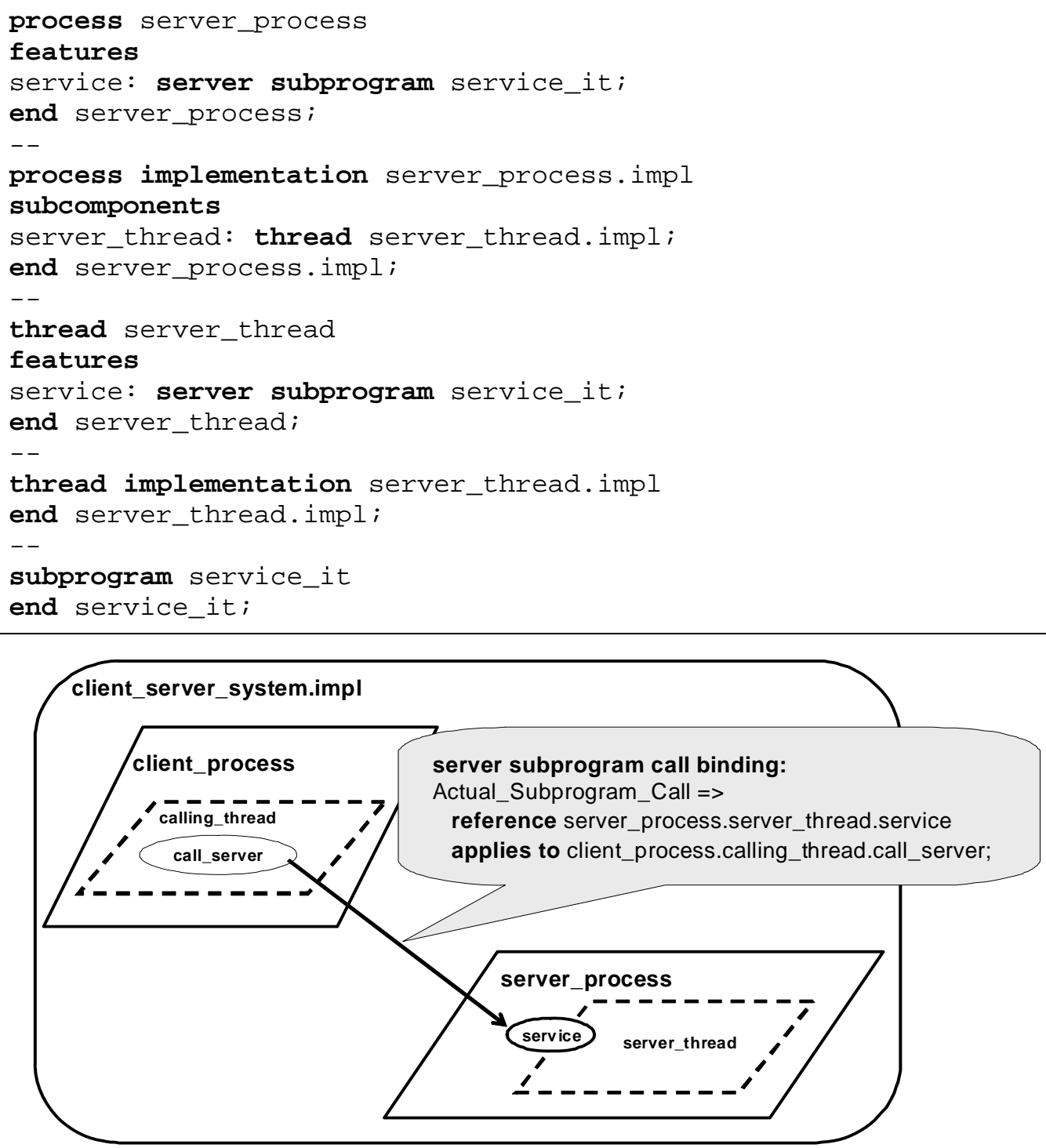

\subsubsection{Properties}

Subprogram calls properties identify the allowed and actual server subprograms involved in a remote server subprogram call. In addition, these properties can be used to specify the allowed and actual binding of the calls to physical elements that support a remote server subprogram call. If no values are assigned to these properties, the subprogram call is a local call to a server subprogram. ${ }^{22}$

22 In the AADL standard, the subprogram calls of all threads must either be local calls or be bound to a server subprogram whose thread is part of the same mode, in a completely instantiable system [SAE 06a]. 


\subsection{Data Exchange and Sharing in Subprograms}

A subprogram can receive and provide data through a variety of mechanisms including

- parameter (passing by value)

- access (passing by reference)

- global/static (shared) data

These diverse and often implicit aspects of data that are followed in programming languages can be modeled and explicitly documented in an AADL representation through parameters, access features, and their associated connections.

\subsubsection{Data Exchange by Value: Parameters and Connections}

A parameter represents call and return data values passed into and out of a subprogram. These exchanges by value are declared as typed data features in the type declaration of a subprogram, similar to data port declarations. Parameter connections are used to describe the flow of data into and out of a subprograms and the data flow through a sequence of subprogram calls within a thread. These connections can be useful in a comprehensive flow analysis when used in conjunction with flows declarations. For more detail on the use of parameters in flow analysis, see Section 10 (Flows).

Table 8-13 presents textual and graphical representations of the parameters and the parameter connections associated with a calls sequence within a thread. In a graphical representation

- parameters are represented as solid arrows $(\bullet)$, like data ports

- parameter connections are shown as solid lines (-) between parameters or between a parameter and a port (on a containing thread of the subprogram call)

- subprogram calls are represented by ovals $(\odot)$ labeled with the call (e.g., scale) and called subprogram type

- calls sequence is indicated by an arrow with an open arrow head $(\rightarrow$ ) (Alternatively, a calls sequence can be specified by the ordering of the calls from the left to the right.)

Notice that the in event data port in_data of the thread scale_data is connected to the parameter in_parameter of the subprogram scale. Parameters can be connected to in data port, out data port, and event data port. 
Table 8-13: Example Parameter Connections
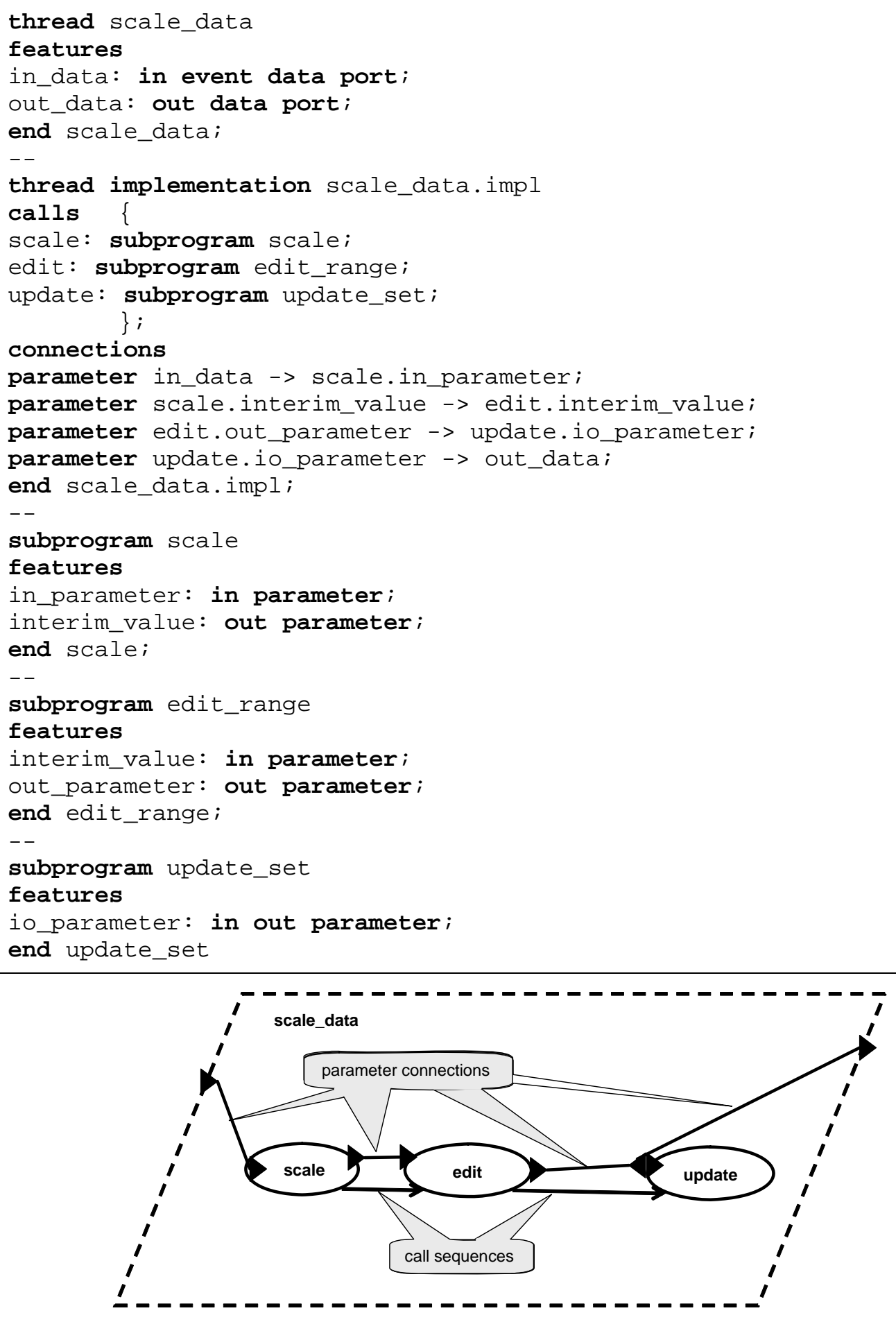

\subsubsection{Data Passing by Reference and Global Data}

The flow of data into and out of a subprogram can involve references to data (e.g., pointer values) or access to common data values (i.e., global or static data), rather than 
explicit data passing. These data reference mechanisms are described through data requires/provides data access declarations in an AADL model.

For example, consider the annotated pseudocode and corresponding AADL textual representation in Table 8-14. In the pseudocode, examples of subprogram calls with data reference and the use of global data are shown. In the Passing by reference section of pseudocode, the function scale modifies data (referenced with the pointer p1) using the scale factor v1. In the second implementation of scale (the Global variable section of Table 8-14), a parameter data value (the scale factor) is passed and a common data element $r a w \_d a t a$ is scaled.

Within AADL, both of these options are represented with $v 1$ as a parameter, whereas the pointer $\mathrm{p} 1$ and the common data $\mathrm{raw}$ _data are represented as a data access feature of the subprogram scale. The thread processing has a call to the subprogram scale. A corresponding AADL representation for the Global variable pseudocode explicitly shows the thread receiving the data value for v1 through the in data port scalar and using that value in the subprogram call, as indicated by the parameter connection VC1 in the thread. In contrast, the pointer reference to the data to be scaled is represented as a data access in the subprogram type declaration for scale. The explicit reference to raw_data in the subprogram scale is the requires statement in the thread type declaration. The AADL specification allows an implementation using either option shown in pseudocode.

Table 8-14: $\quad$ Examples of Passing by Reference and Global Data

\begin{tabular}{|c|c|}
\hline Pseudocode & AADL Representation \\
\hline $\begin{array}{l}\text { Passing by reference: } \\
\text {..... } \\
\text { scale (v1, p1) } \\
\text { v1 is a real that is } \\
\text { the scale factor. } \\
\text { p1 is a pointer to a } \\
\text { data set 'raw_data' } \\
\text { that is to be scaled. } \\
\text {... } \\
\text { processing that calls } \\
\text { the subprogram: } \\
\text {... } \\
\text { call scale (v1, p1); } \\
\text {... }\end{array}$ & $\begin{array}{l}\text { subprogram scale } \\
\text { features } \\
\text { v1: in parameter real; } \\
\text { p1: requires data access raw_data; } \\
\text { end scale; } \\
\text {-- } \\
\text { data raw_data } \\
\text { end raw_data; } \\
\text {-- } \\
\text { data real } \\
\text { end real; } \\
-- \\
\text { thread processing } \\
\text { features } \\
\text { scalar: in data port real; } \\
\text { p1: requires data access raw_data; } \\
\text { end processing; } \\
\text {-- }\end{array}$ \\
\hline
\end{tabular}


Table 8-14: $\quad$ Examples of Passing by Reference and Global Data (cont.)

\begin{tabular}{|c|c|}
\hline $\begin{array}{l}\text { Global variable: } \\
\text {... } \\
\text { variable and } \\
\text { processing } \\
\text { definitions: } \\
\ldots . \\
\text { real: raw_data; } \\
\ldots \\
\text { scale(v1) } \\
\quad\{\quad \text { x := } \\
\text { raw_data; } \\
\quad \text { \} } \\
\text { processing that } \\
\text { calls the } \\
\text { subprogram: } \\
\ldots \\
\text { call scale(v1); } \\
\text {... }\end{array}$ & $\begin{array}{l}\text { thread implementation processing.impl } \\
\text { calls \{ } \\
\qquad \text { scale_it: subprogram scale; } \\
\text { connections } \\
\text { VC1: parameter scalar -> scale_it.v1; } \\
\text { PC1: data access p1 -> scale_it.p1; } \\
\text { end processing.impl; } \\
\text {-- } \\
\text { process data_management } \\
\text { features } \\
\text { scalar: in data port real; } \\
\text { end data_management; } \\
\text {-- } \\
\text { process implementation data_management.impl } \\
\text { subcomponents } \\
\text { r_data: data raw_data; } \\
\text { data_processing: thread processing.impl; } \\
\text { connections } \\
\text { C1: data port scalar -> data_processing.scalar; } \\
\text { C2: data access r_data -> data_processing.p1; } \\
\text { end data management.impl; }\end{array}$ \\
\hline
\end{tabular}

\subsubsection{Method Calls in AADL}

Calls to object methods can be represented in AADL as calls to subprogram features of a data component. Consider the pseudocode in Table 8-15 where the method errorTotal of the class ErrorLog returns an integer value that is the total number of errors currently in the log. The corresponding AADL representation involves the declaration of an enclosing process implementation that establishes instances of the thread monitor and the data component ErrorData, as well as the required data access of the thread monitor to ErrorData. The implementation of the thread monitor involves the call sequence to subprograms errorTotal and reset. The integer type return value for errorTotal is represented as the out parameter total. The data access connections are shown graphically in the figure of Table 8-15 and indicate the subprogram and thread access to ErrorData.

Table 8-15: $\quad$ Methods Calls on an Object

\begin{tabular}{|c|c|}
\hline Object-Oriented Pseudocode & AADL Representation \\
\hline $\begin{array}{l}\text { class ErrorLog }\{ \\
\text { int errorTotal ( ) }\{ \\
\ldots \\
\quad \text { void reset () }\{ \\
\ldots . \\
\ldots\end{array}$ & $\begin{array}{l}\text { process implementation } \\
\text { maintenance.control } \\
\text { subcomponents } \\
\text { monitor: thread monitor.errors; } \\
\text { ErrorData: data ErrorLog; } \\
\text { connections } \\
\text { C1: data access ErrorData -> } \\
\text { monitor.log_access; } \\
\text { end maintenance.control; }\end{array}$ \\
\hline
\end{tabular}


Table 8-15: $\quad$ Methods Calls on an Object (cont.)

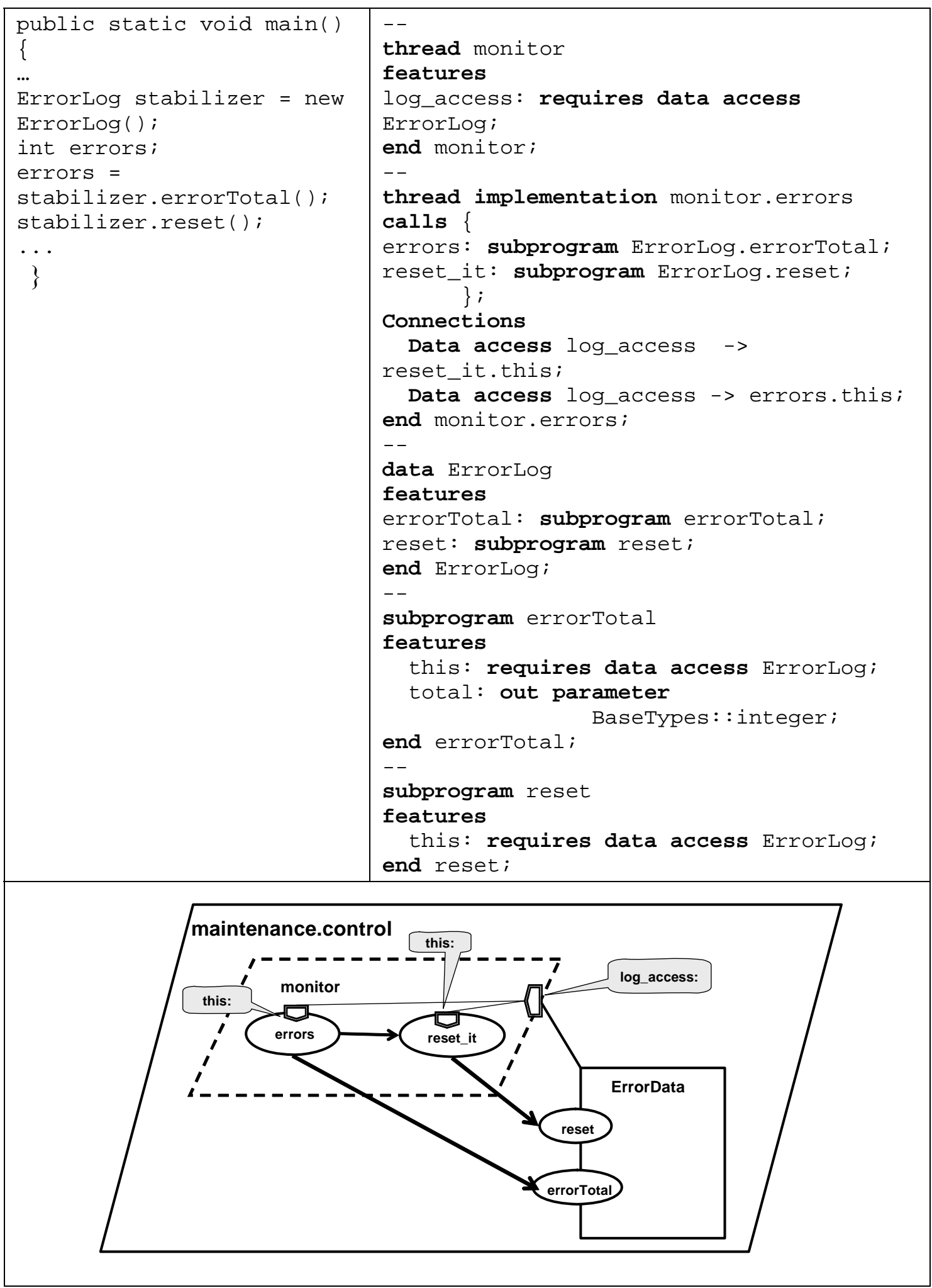




\section{Modes}

A modes abstraction is an explicitly defined configuration of contained components, connections, and property value associations. Modes represent alternative operational states of a system or component. For example, modes for a cruise control system may be $\{$ initialize, disengaged, engaged\}, where each of these modes may involve different sets of processes, executing threads, or active connections (e.g., in the initialization mode there are no connections to sensors).

Modes may specify different calls sequences to be used in a thread or subprogram. Modes also may represent different logical states of any component, such as a thread or subprogram, for which different property values apply. For example, under different modes a thread may have different execution times to represent an algorithm that can execute with different levels of precision. Modes may also represent different hardware configurations such as processors that are active at any one time.

\subsection{Modal Specifications}

Modes are represented as states within a state machine abstraction. Each distinct configuration of a component is identified as one mode (state) within the modal state machine abstraction for the component. The configuration that defines each mode and the events that cause the transitions in the behavior of the component must be specified. Each modal state machine must have at least two modes, one of which must be declared as the initial mode for the component.

Modes can be used to represent alternative system configurations in a variety of ways. They can establish

- alternative configurations of active components and connections and the transitions among these configurations

- variable call sequences within a thread

- mode-specific properties for software or hardware components

\subsubsection{Modal Configurations of Subcomponents and Connections}

Table 9-1 presents both textual and graphical representations of modes transition specifications for a simplified controller thread within a cruise control system. In this example, mode transitions are triggered by external events. Only the relevant ports are shown in the type declaration for the thread control. Neither type nor implementation declarations are complete. The graphic shows the mode transition view for the thread. 
There are two modes, idle and controlling, and three event ports in this example. The idle mode is the initial mode. The event brought into the thread by event port Cc_engage results in a mode transition to the controlling mode (the thread configuration that provides the functionality to maintain a set speed). The event carried through the event port cc_resume_e1 also results in a switch to the controlling mode using the previous value of the speed setting. Event port cc_brake results in an exiting of the controlling mode to the idle mode.

Table 9-1: Sample Graphical and Textual Specifications for Modes
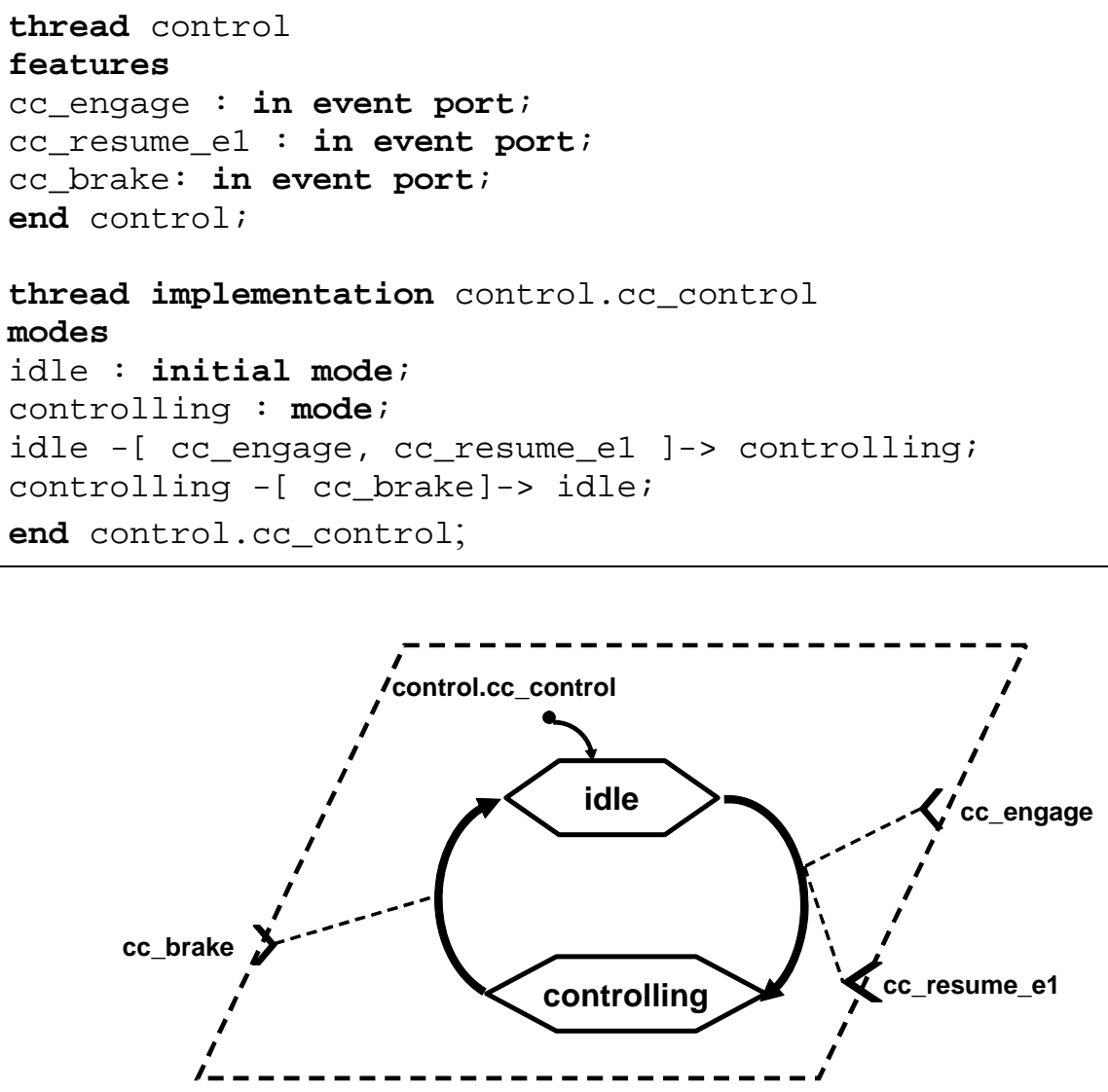

The example in Table 9-2 shows a multimode process where internal events result in mode changes of a process. In the textual specification for the process

control_algorithms.impl, the modes section defines the two operational modes of ground and flight and the transitions between them. The transitions are triggered by out event ports from the thread controller that is a subcomponent of the process implemenation control_algorithms.impl. The specification for the process implementation includes in modes clauses that define the subcomponents and connections active in each mode. 
In the upper right portion of the figure in Table 9-2, a graphic shows the modes and their transitions that are triggered by the events from the controller thread. In that figure, the flight mode configuration is shown in black and the ground mode is shown in gray. This distinction illustrates that the ground_algorithms thread and its connections are not part of the flight mode.

Table 9-2: Modes Example

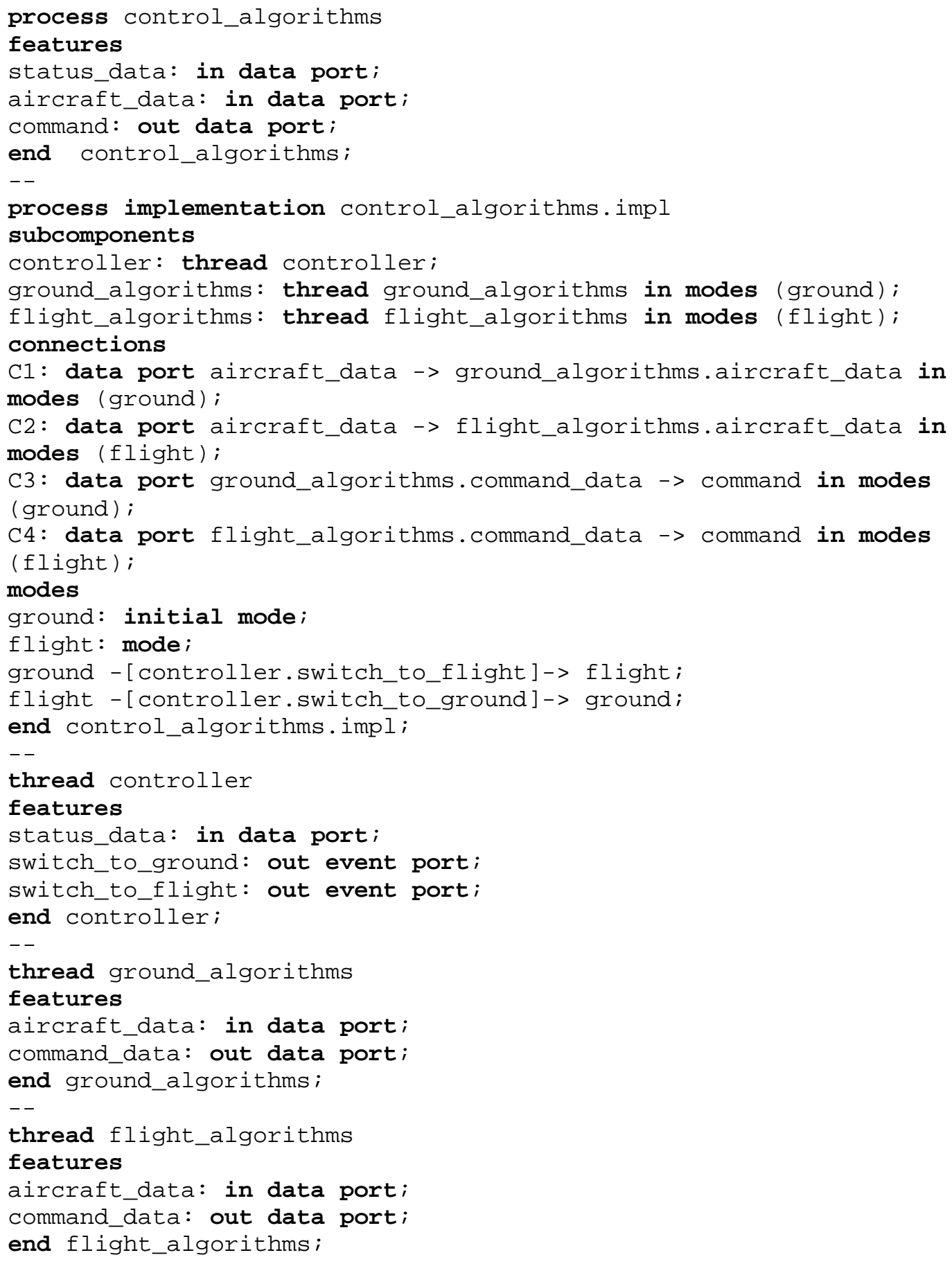


Table 9-2: Modes Example (cont.)

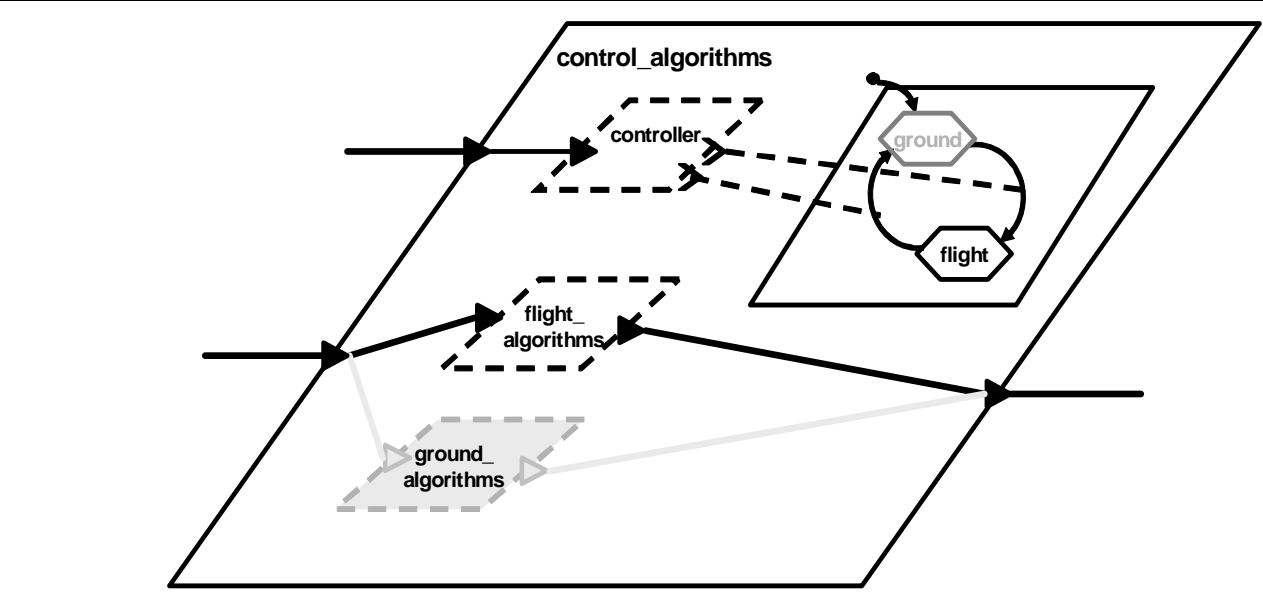

\subsubsection{Modal Configurations of Call Sequences}

Alternative calls sequences can be specified using modes. The example in Table 9-3 shows a monitor thread that checks software and hardware and reports anomalies. The thread employs a sequence of calls to subprograms when the thread is in the nominal mode. When an error is detected, an error_condition is signaled through the event port error_event. This signal results in a mode switch and changes the subprogram calls sequence of the thread.

Table 9-3: Mode-Dependent Call Sequences

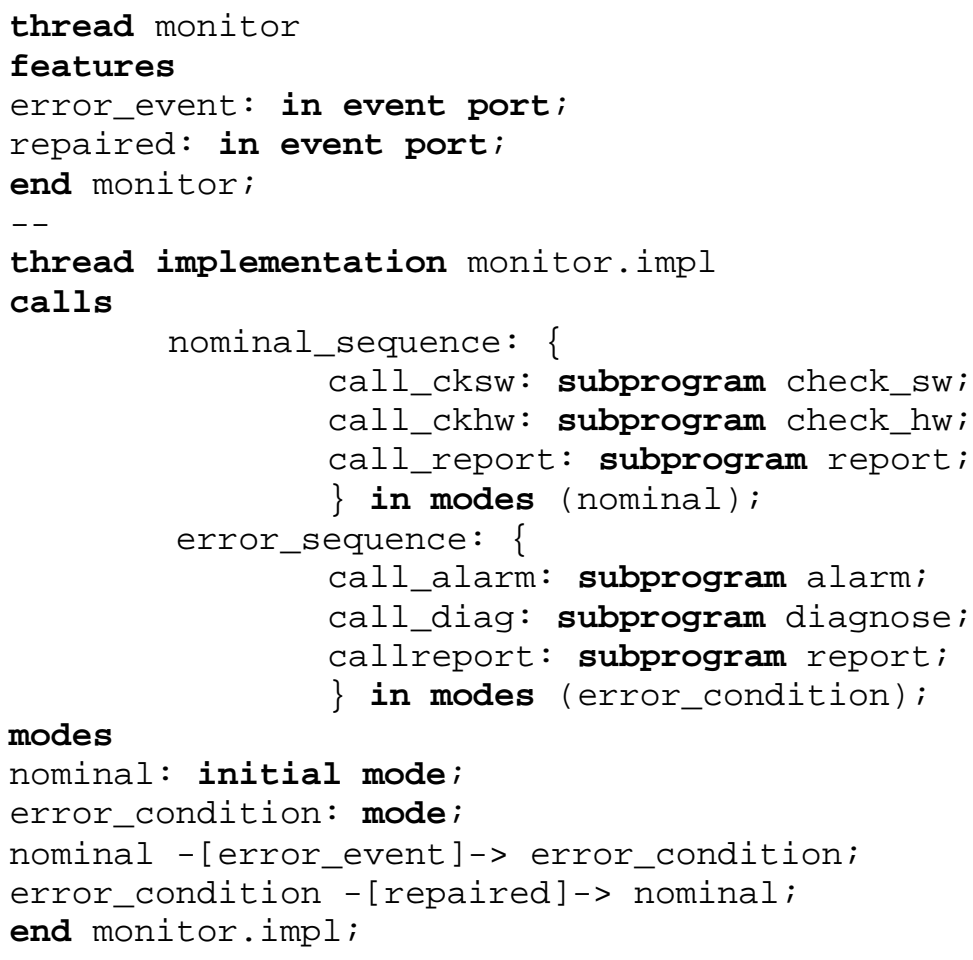




\subsubsection{Mode-Specific Properties}

Property values assignments can be mode-dependent. These mode-specific property associations can be used to define alternative characteristics and behavior for components. For example, consider the partial specification in Table 9-4 that has a modified version of the process implementation for control_algorithms. impl shown in Table 9-2. In this example, the controller thread has a different execution time for the ground mode than for the flight mode.

Table 9-4: Mode-Specific Component Property Associations

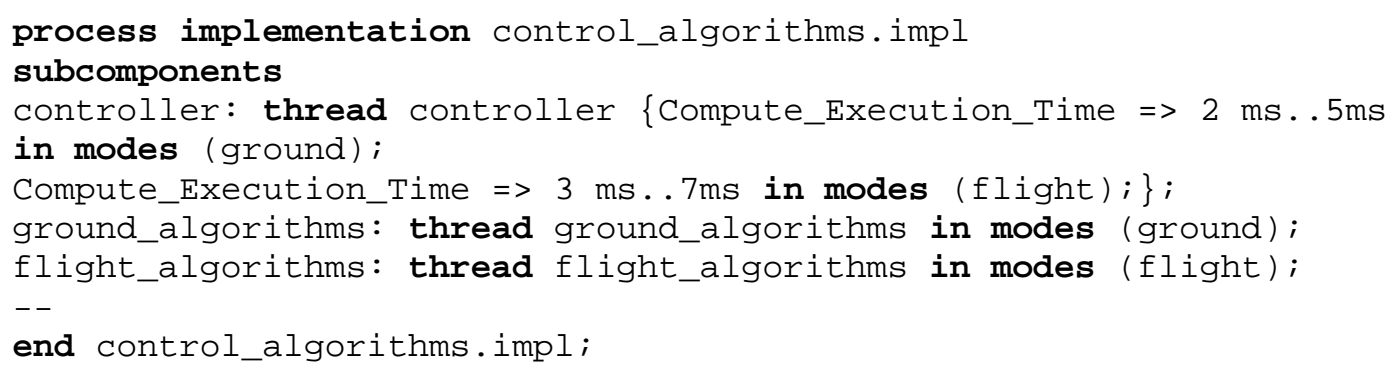




\section{Flows}

AADL flows specification capabilities enable the detailed description and analysis of an abstract information path through a system. A complete path for an abstract information flow - an end-to-end flow implementation - begins at a source component and terminates at a sink component. The specification of an end-to-end flow involves the declaration of the elements of the flow (sources, sinks, and paths) and explicit implementation declarations that describe the details of a complete path through the system.

A source component of a flow is characterized by the feature (e.g., port, port group, or parameter) through which the flow emerges from the component. Similarly, a sink component of a flow is characterized by the feature through which the flow enters the component and terminates. Details of a flow path are described by identifying the entry and exit features of each intermediary component and subcomponent involved in the flow.

\subsection{Flow Declarations}

Flows are directional. To specify a complete flow, declarations in component types and implementations are required. For a component type, flows declarations designate a

- source: a feature of a component

- sink: a feature of a component

- flow path: a flow through a component from one feature to another

Table 10-1 shows a partial specification for a simplified cruise control system with flow source, flow sink, and flow path declarations within component type declarations. Notice that the flow path brake_flow through the system component cruise_control has an in event data port as its origin and an out data port as its termination feature. The lower portion of the table includes a graphical representation of the declarations.

Table 10-1: $\quad$ Flow Declarations within a Component Type Declaration

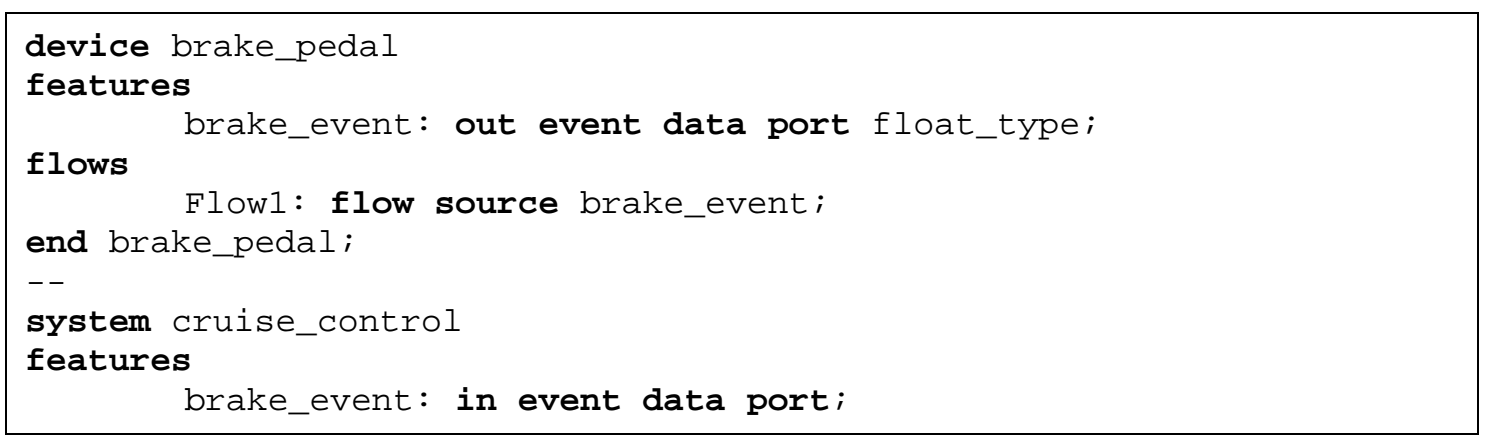


Table 10-1: Flow Declarations within a Component Type Declaration (cont.)

throttle_setting: out data port float_type;
brake_flow: flow path brake_event $->$ throttle_setting;
end cruise_control;
device throttle_actuator
features throttle_setting: in data port float_type;
flows Flow1: flow sink throttle_setting;
end throttle_actuator;

\subsection{Flow Paths}

Within a component implementation, flow declarations define the details of

- flow paths through a component

- end-to-end flows within the component

\subsubsection{Flow Path through a Component}

A flow path through a component consists of alternating sequences of paths through and connections among subcomponents within the component. This path begins and ends at features of the component type and is a realization of the corresponding flow path declared in the component's type declaration. Table 10-2 shows the flows implementation declarations through the component cruise_control.impl for the flow path brake_flow declared in the type declaration cruise_control of Table 10-1. It also shows a graphical representation of the flow path.

The flows implementation originates at the brake_event event data port and proceeds through to the data port throttle_setting. The flow involves the connections $\mathrm{C} 1, \mathrm{C} 3$, and $\mathrm{C} 5$ within the component implementation cruise_control.impl, as well as the paths through the subcomponents of that implementation. Notice that the nature of the data within the flow changes and involves event data ports as well as data ports. 
Table 10-2: $\quad$ Flow Implementation Declarations through a Component
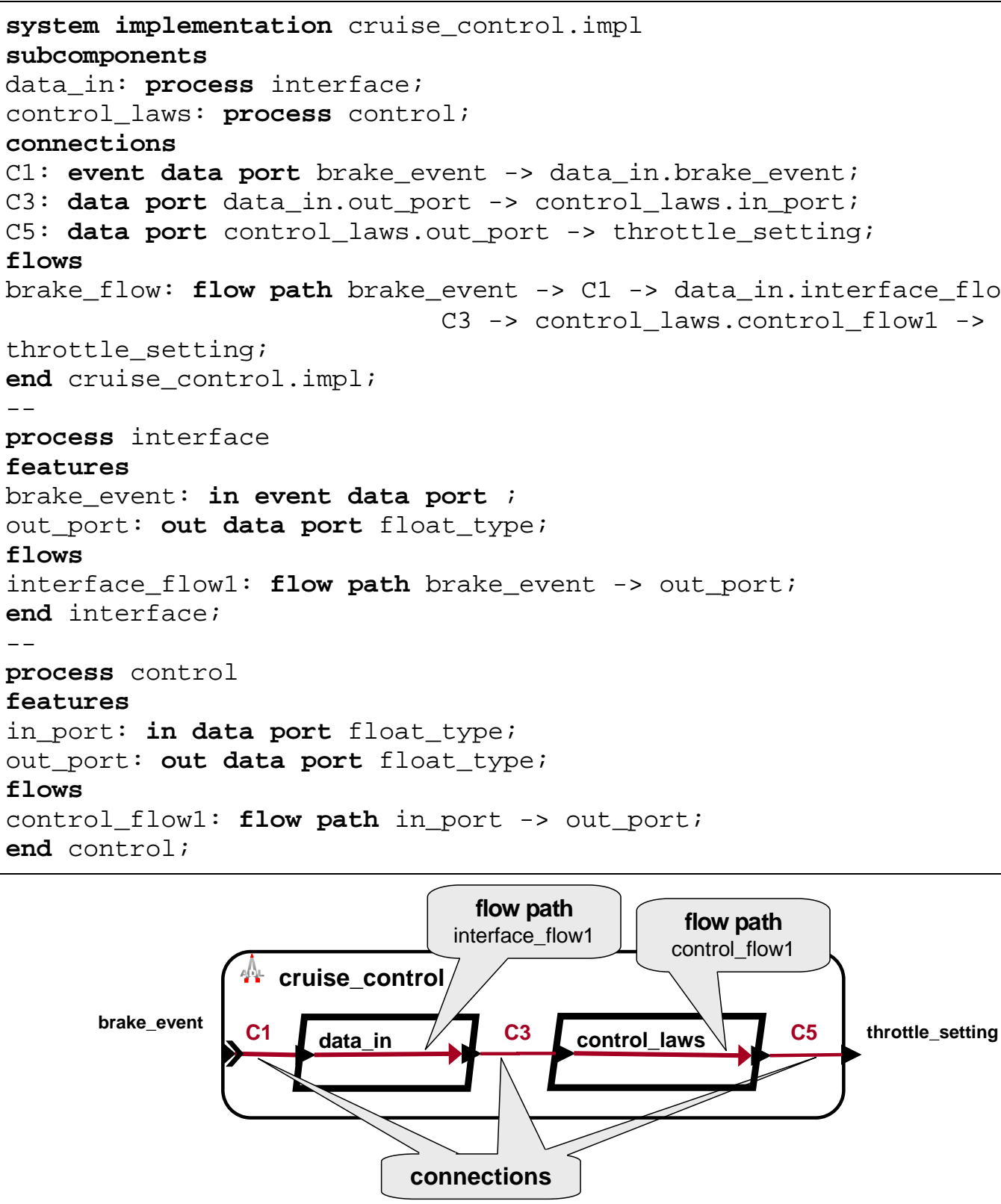

\subsubsection{End-to-End Flow within a Component}

An end-to-end flow within a component involves the declaration of a path from a flow source to a flow sink within the component. The partial specification in Table 10-3 illustrates this type of declaration: an end-to-end flow is defined between the source Flow1 in the device component brake_pedal and the sink Flow1 in the device component throttle_actuator. 
Table 10-3: $\quad$ An End-to-End Flow
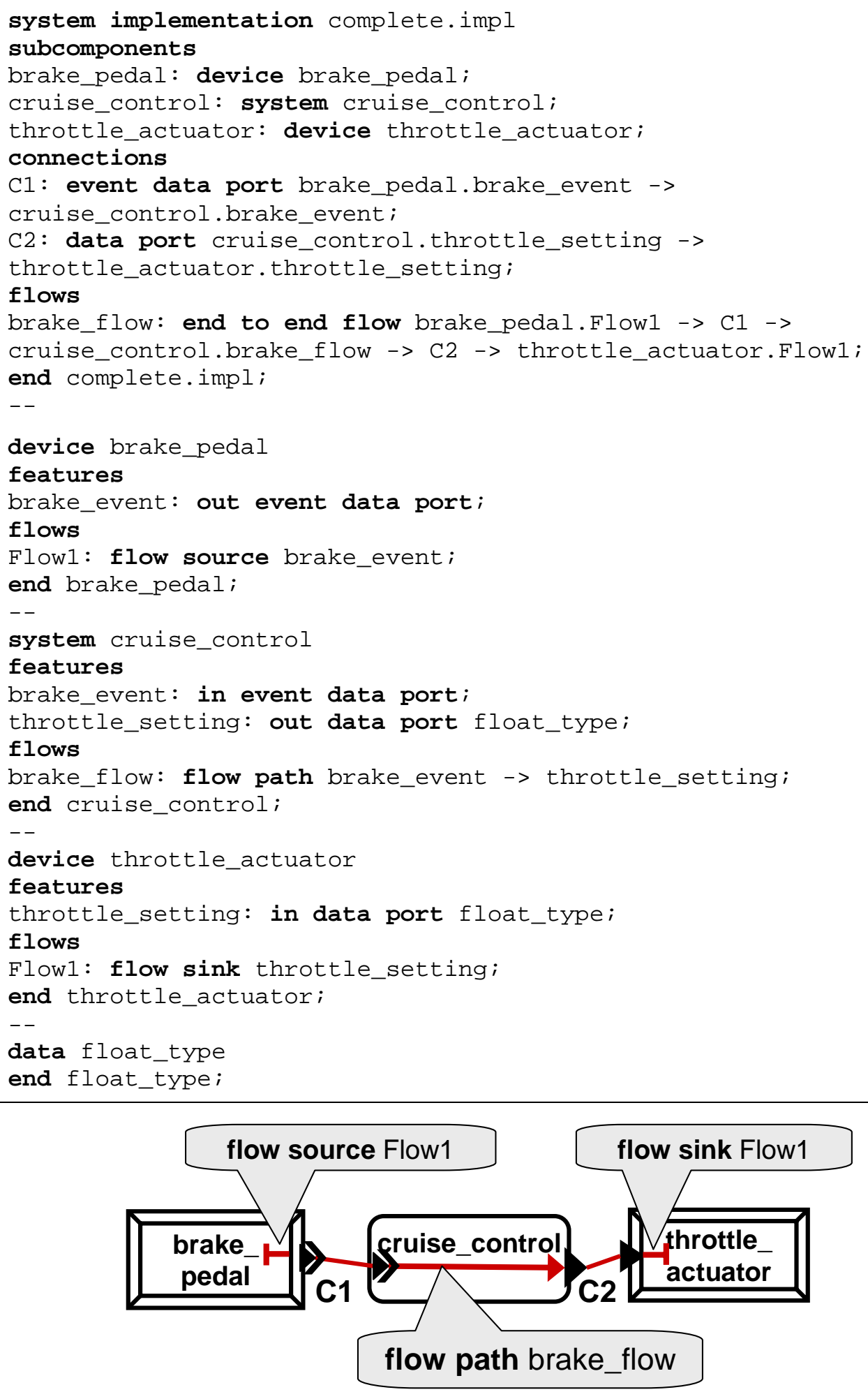


\section{Properties}

Properties provide descriptive information about

- components

- subcomponents

- features

- connections

- flows

- modes

- subprogram calls

A property has a name, type, and an associated value. Properties can be assigned values through property association declarations.

There are built-in property types and predeclared properties in the AADL standard. Collectively, these properties and property types encompass common attributes for the elements of the language. For example, a predeclared property of a port is Required_Connection, which is of type aadlboolean and has a value of true or false. ${ }^{23}$ Its predeclared (default) value is true. However, a property association can assign the value false, allowing the port to be unconnected. A summary of AADL built-in property types is included on page 122 in the Appendix.

In addition to providing predeclared properties and built-in property types, AADL also permits the defining of new properties and property types. For example, to define a new property (e.g., Priority) for a thread, a user would declare a property name, type, and association of the new property. The property type declared for a new property may be a built-in type (e.g., aadlinteger), or a new type can be declared using a property type declaration.

\subsection{Property Declarations}

The declarations relating to properties are listed below.

- property association (Section 11.2): assigns a value or list of values to a named property

${ }^{23}$ Required_Connection is included in the predeclared property set named AADL_Properties that is part of every AADL specification [SAE 06a]. 
- $\quad$ property set (Section 11.3): defines a named collection of property types, names, and constants

- Property type (Section 11.4) defines a property type and specifies the set of acceptable values for properties of that type.

- Property name (Section11.5) defines a property by declaring a name, identifying a type for the property, and applying it to a category of element within the specification (i.e., mode, port group, flow, port, server subprogram, or connection).

- Property constant (Section 11.6) defines a name for a property value that can be referenced in property expressions wherever the value itself is permissible.

Property name, property type, and property constant declarations must be contained within a property set declaration.

\subsection{Assigning Property Values}

A property can be assigned a value or a list of values through a property association declaration. Property values can be associated with properties directly within individual component declarations, through an inherited value or an explicit contained property association referencing elements within a hierarchal component. In addition, property associations can be declared as being mode- or platform-binding specific.

\subsubsection{Basic Property Associations}

Property associations can be included within the properties section of component type or implementation declarations or within declarations for subcomponents, features, connections, flows, modes, and subprogram calls and their refinements.

Sample component property association declarations are shown in Table 11-1 where an implementation speed_data of the thread type data_processing is declared with associations for two standard properties. The Period property is assigned a single value of $100 \mathrm{~ms}$. The Compute_Execution_Time assigned value is a range. In addition, the in data port declaration sensor_data includes a property association that declares the port need not be connected, and the thread subcomponent declaration for data_processing includes a property association declaring the initialization execution time range for the thread (1 ms . . $2 \mathrm{~ms}$ ).

Table 11-1: $\quad$ Basic Property Association Declarations

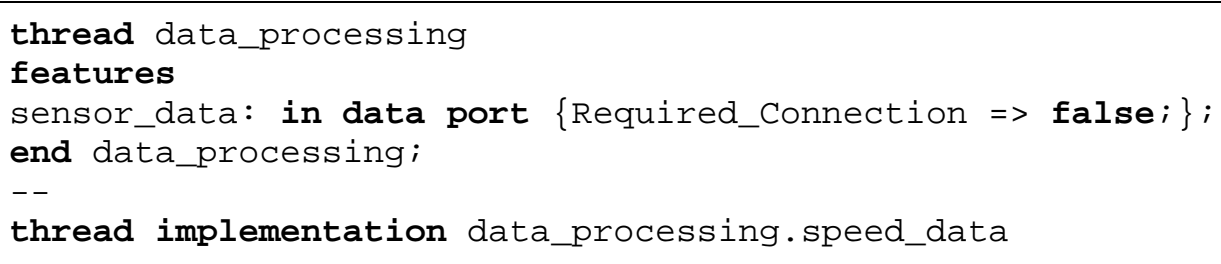


Table 11-1: Basic Property Association Declarations (cont.)

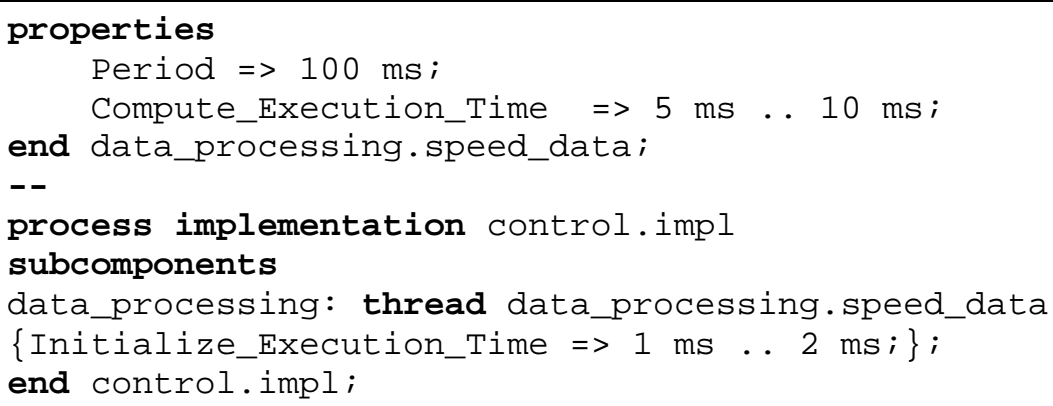

Access property associations are used to detail the character of subcomponent access, both requires and provides. Table 11-2 shows two access property associations, where the process control requires read_only access to set point data data_sets.set_points and provides read_write access to its internal error logs. This is a modification of an example from Table 8-7.

\section{Table 11-2: $\quad$ Sample Access Property Associations}

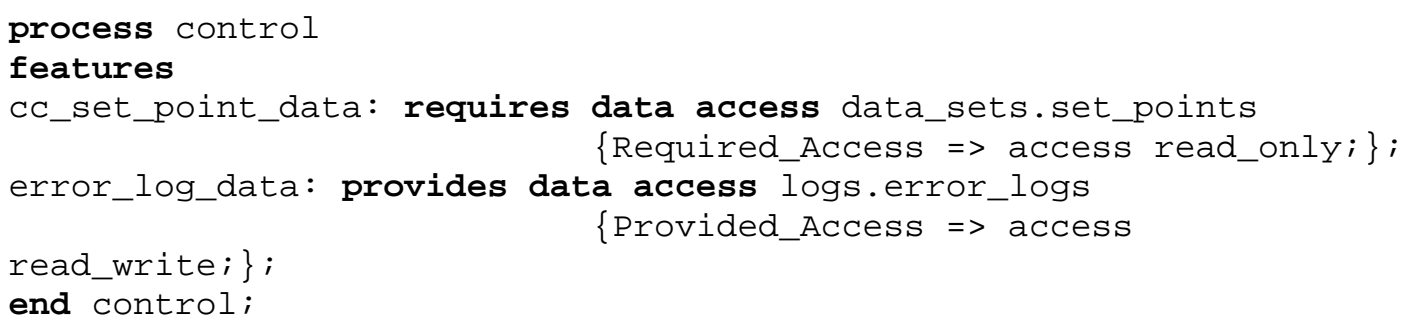

\subsubsection{Contained Property Associations}

Property associations for individual components have been shown in earlier examples (e.g., Table 11-1). These declarations assign values for instances of the component. However, explicit property associations may be omitted for a number of the elements of an individual component. In these cases, values can be assigned through contained property association declarations or inherited from declarations higher in the component containment hierarchy.

A contained property association can be used to assign a property value to subcomponents, features, flows, connections, or modes defined within a component. A value can be assigned to an element that is deeply nested within the component. In addition, with contained property associations, configuration parameters for a system can be defined at a single point (e.g., at the highest point possible in the component hierarchy). In that way, the parameters provide a centralized set of properties and values for elements of a model that can readily be identified, adjusted, and reviewed.

An explicit contained property association is declared using an applies to clause that specifically identifies an element within the component. The identification path to the element consists of a dot-separated sequence of zero or more subcomponent identifiers 
followed by the identifier of the subcomponents, features, flows, connections, or modes identifier to which the property association applies. Consider the partial specification in Table 11-3 that shows the relevant type and implementation declarations for a simplified cruise control system. The property associations within the system implementation declaration for cc_complete.impl are property associations for the execution time for the compute entry point of a contained thread control_algorithm and the required connection value for a data port of the contained thread adjust.

Table 11-3 shows two contained property associations within the system implementation cruise_control.impl. In the first association, the computation time for the compute entry point of the subcomponent thread control_algorithm is assigned the range of $2 \mathrm{~ms}$. . $5 \mathrm{~ms}$. The thread control_algorithm is contained within the process control_laws that is a subcomponent of the system cruise_control. In the second association, the Required_Connection property is assigned the value false for the out data port of the contained thread adjust.

Table 11-3: $\quad$ Contained Property Associations

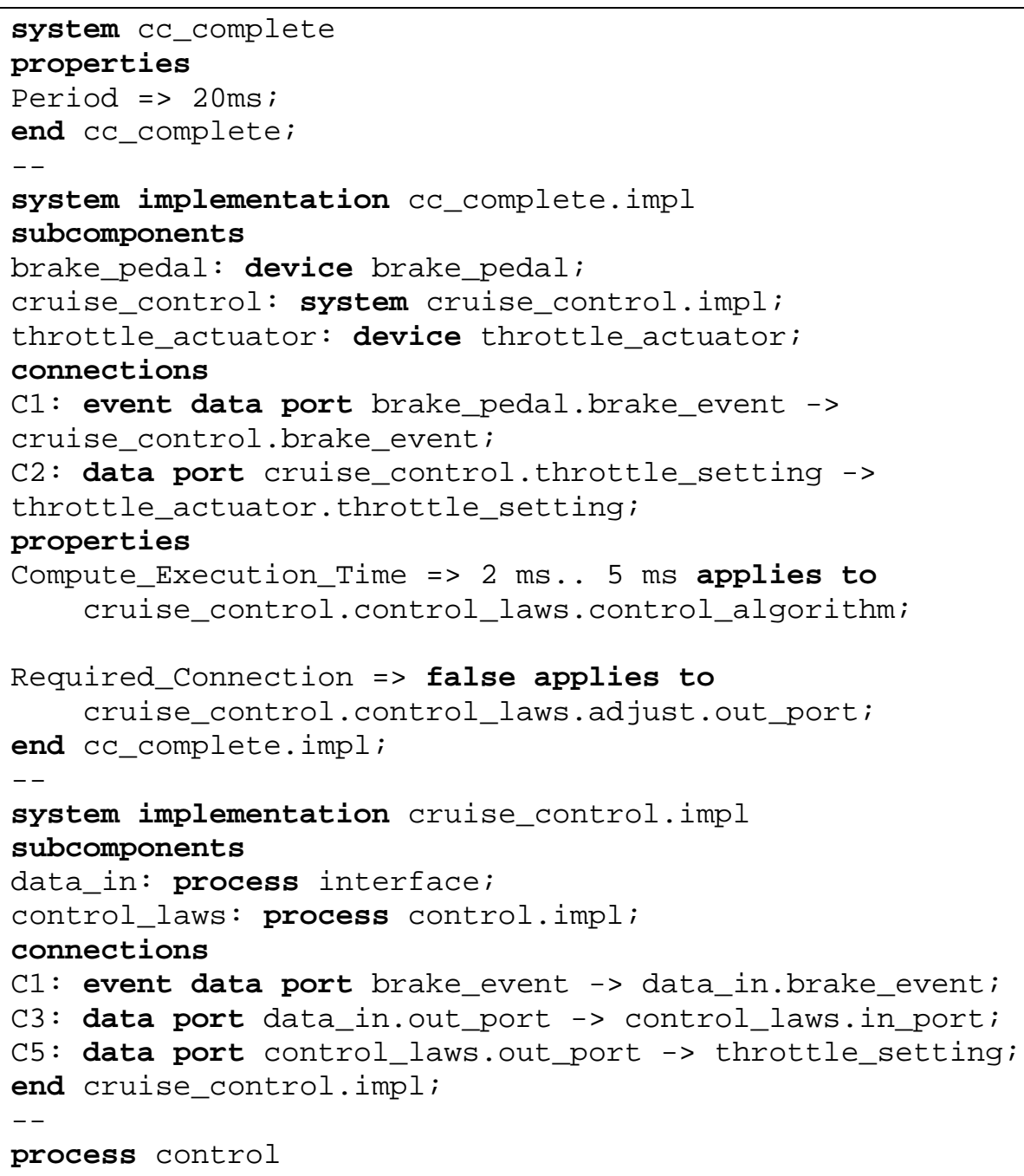


Table 11-3: $\quad$ Contained Property Associations (cont.)
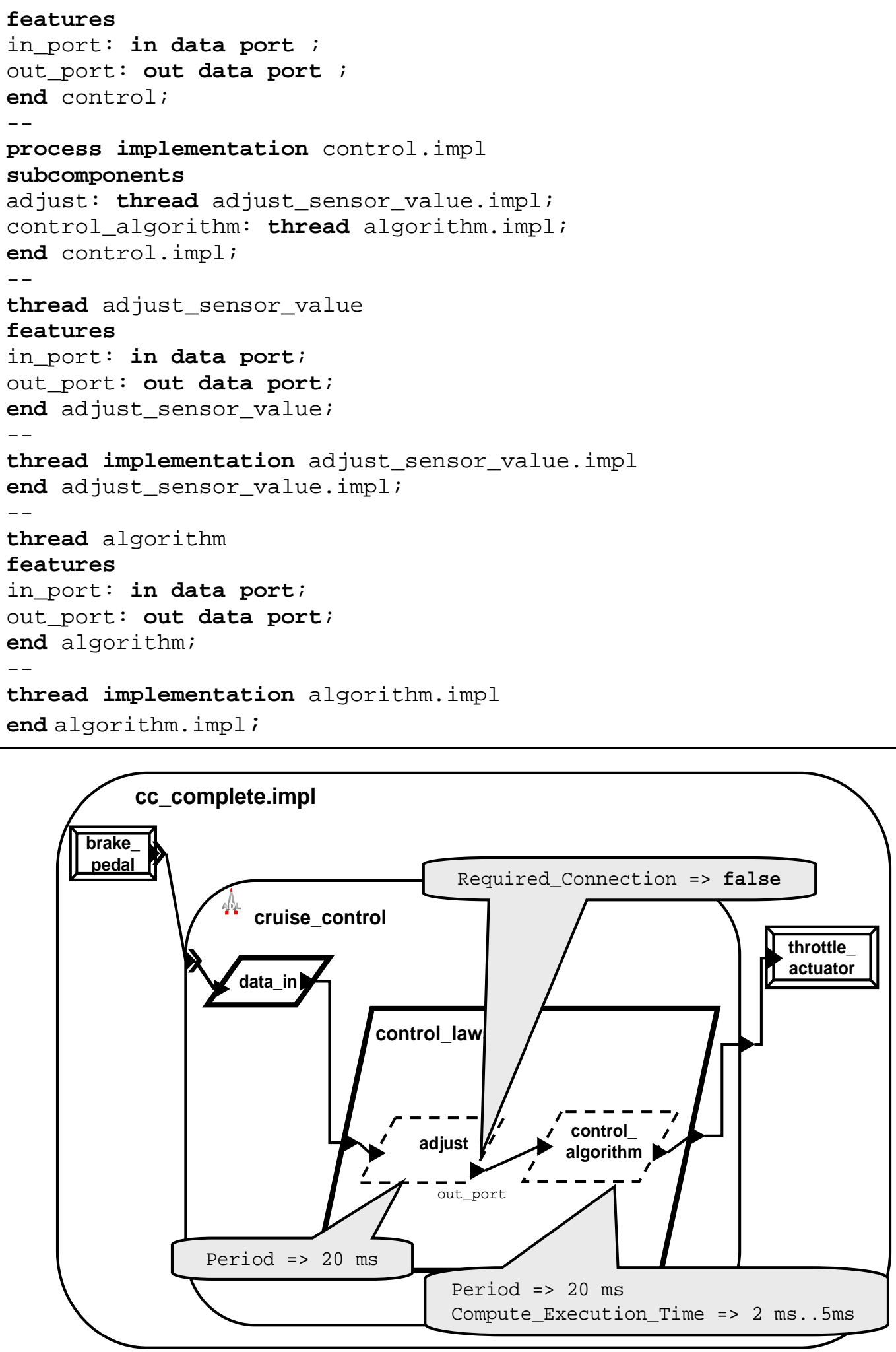
Contained property associations are required when a property value involves a reference to another part of a model. For example, the binding property of a thread must refer to the processor to which it is bound. However, that reference is represented as a path relative to the location at which the property association is specified. Thus, the property association must be declared as contained property association attached to a model component that is the common parent of the component being referenced and the component to which the property value belongs.

An example of a contained property association across a component hierarchy is shown in Figure 11-1 for the property Allowed_Processor_Binding. The property association is included in the specification for the system component Avionics_sys and declares that the thread observe can be bound to the processor linux1.

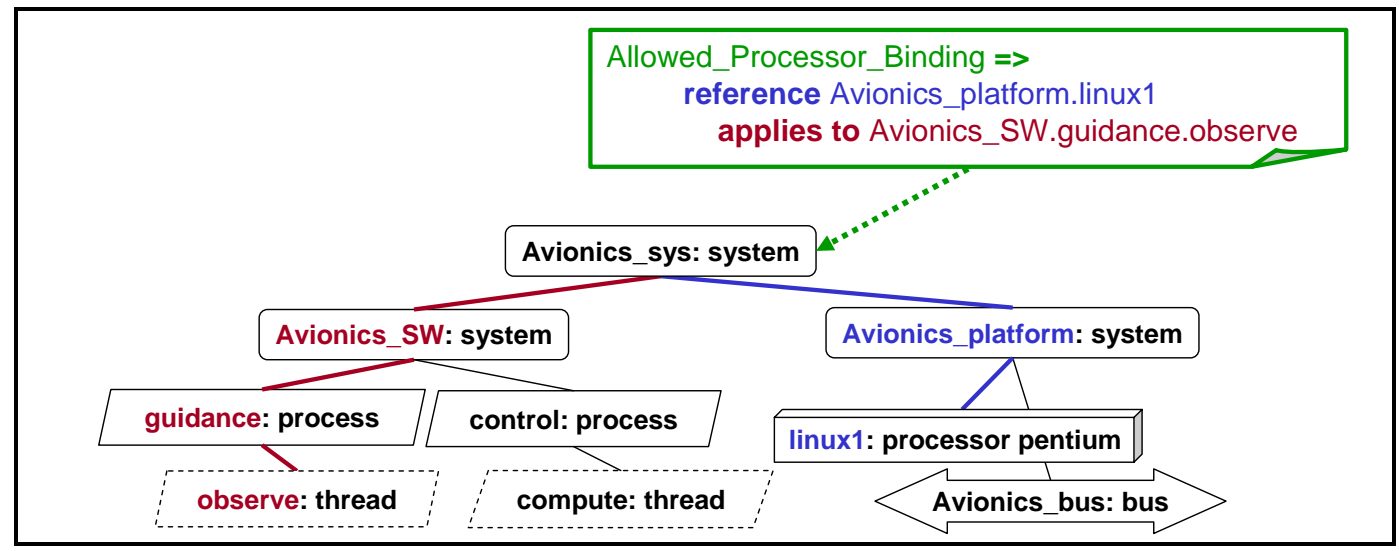

Figure 11-1: Contained Property: Allowed_Processor_Binding

\subsubsection{Inherited Property Associations}

There is an implicit form of a property association that can be declared for contained components. This form involves properties defined with the inherit reserved word. For these properties, a property association declaration within a component is assigned to any subcomponent to which the property applies. For example, a Period property association within a process declaration applies to all of the threads contained within it, unless an individual thread property association declaration assigns a different value to the Period. An example Period property declaration within a system type declaration is shown in Table 11-3. A graphical representation is shown in the lower portion of that table. See Section 11.5 for more information.

One should be cautious in using this implicit property assignment for subcomponents. An inadvertent omission of a specific assignment for a contained component is not readily detectable and may result in an incorrect property value assignment. In the example shown in Table 11-3, Period for the thread adjust defaults to an execution time of $20 \mathrm{~ms}$. If the intention had been to have a Period of $10 \mathrm{~ms}$, there would have to be an explicit declaration for the Period of the adjust subcomponent. 


\subsubsection{Mode or Binding Specific Property Associations}

Property associations can be specialized to specific modes or bindings by declaring this specialization in the property association. For example, the computation time and period property associations from Table 11-3 are declared for a specific processor binding in Table 11-4. Thus, alternative thread execution times and other processor-dependent properties can be declared based upon processor bindings through the in binding declaration. In Table 11-4, the Required_Connection property association is specialized to the initialize mode in the system implementation declaration cc_complete.impl.

Table 11-4: $\quad$ In Binding and In Mode Property Associations

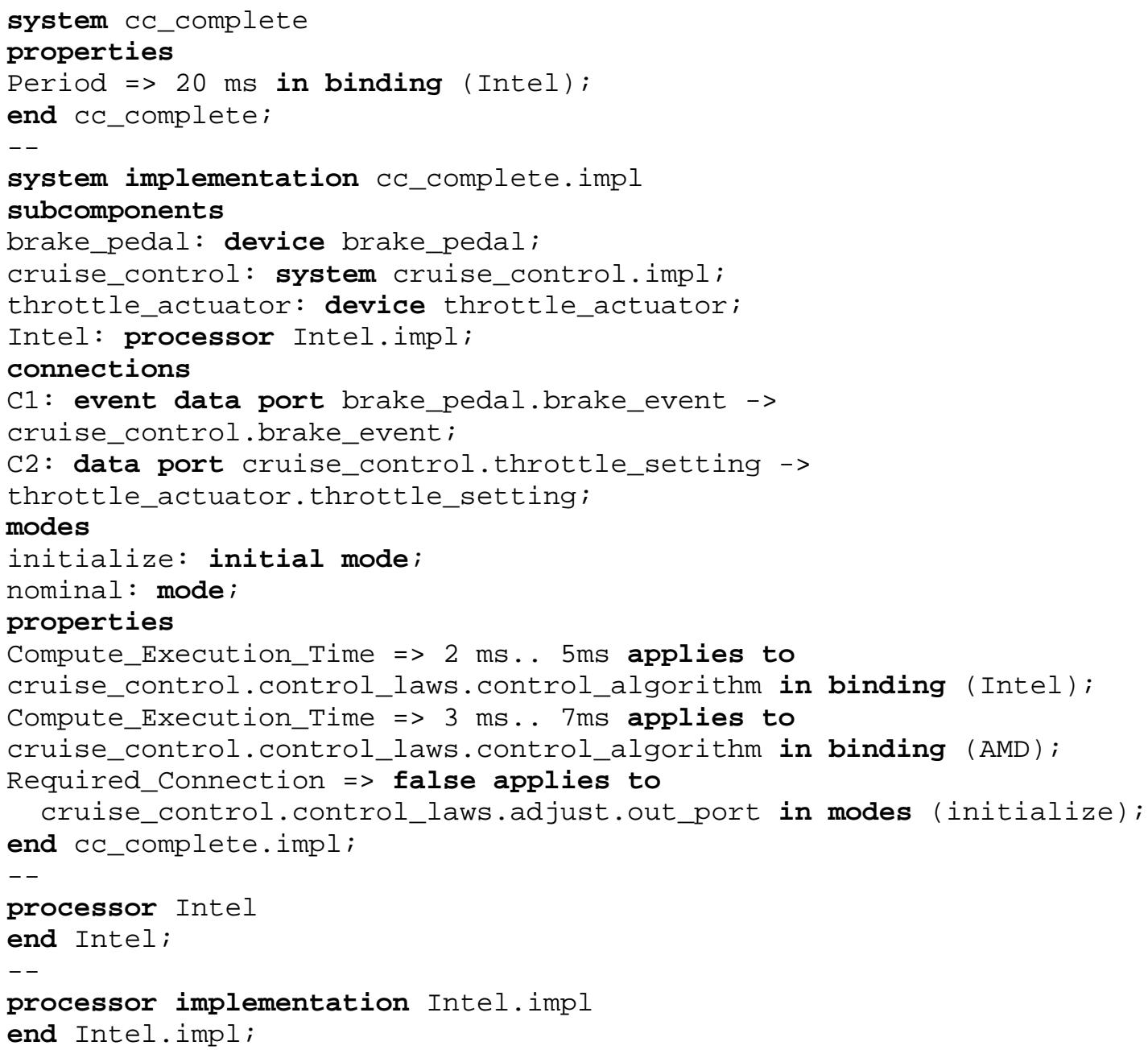




\subsubsection{Property Values}

The values that are assigned to properties can take a variety of forms:

- individual values associated with a basic built-in type like aadlboolean,

$$
\text { aadlstring, aadlinteger, or aadlreal }
$$

- a range of values, as shown in Table 11-4 for execution times

- values with or without units (e.g., Period)

- an enumeration value set (e.g., the Required_Access property)

- values that include model elements as well as explicit component classifiers

- individual values or lists of values

The property type reference allows a property value to refer to a model element according to the containment hierarchy. For example, in Figure 11-1 the Allowed_Processor_Binding declaration references a specific processor in the system hierarchy. The properties of type classifier allow component classifiers to be used as property values. In Table 11-5, the first property association for the property Allowed_Processor_Binding_Class restricts the binding to processors of type PowerPC. The classifier value can be a component implementation or a list of classifier references, as shown in the second property association for the property Allowed_Processor_Binding_Class in the lower part of Table 11-5.

\section{Table 11-5: $\quad$ Classifier Property Types}

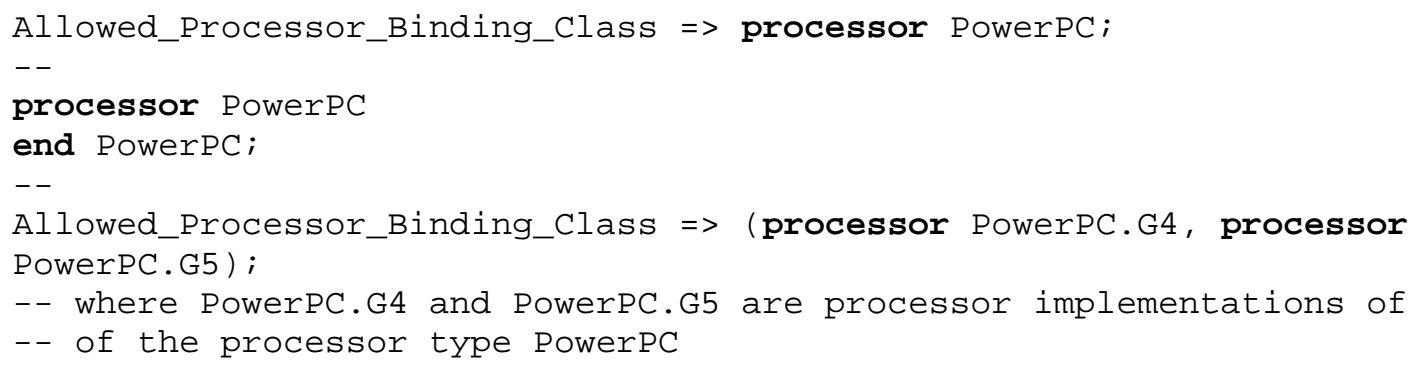

Property value assignments can be indirect and used to centralize the declarations of system parameters. For example, the property associations in Table 11-6 use the keyword value to assign values to the Deadline and Period properties of the thread algorithm. impl. In the property set timing, the property HiRate is defined as a constant of the type Time with a value of $5 \mathrm{~ms}$. Period is assigned the value of HiRate, and the Deadline is assigned the value of Period. Thus, a change in all of these assignments can be accomplished simply by changing the value of HiRate. 
Table 11-6: $\quad$ Property Associations with Value

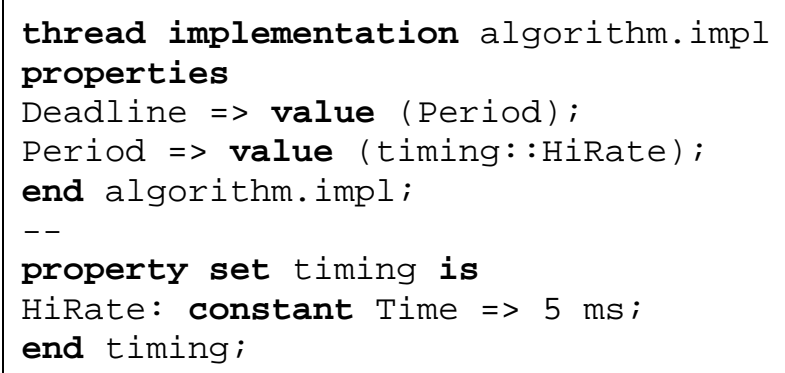

Built-in property types are summarized on page 122 in the Appendix. Details on declaring additional property types are discussed in Section 11.4.

\subsection{Defining New Properties}

A property set is a named collection of property type, property name, and property constant declarations. A named property set can be used to augment a general specification or defined as part of an AADL annex.

Table 11-7 shows the form and content of a sample property set declaration set_of_faults and includes examples of property name, property type, and property constant declarations. The property named comm_error_status is defined as a property of type aadlboolean (true or false) that applies to system and device components. A property type Speed_Range is defined as a range of real values from $\odot . \odot \mathrm{mph} . .150 . \odot \mathrm{mph}$. The constant Maximum_Faults is defined as the integer value 3 .

For more details on

- $\quad$ property type declaration: see Section 11.4

- property name declaration: see Section 11.5

- property constant declaration: see Section 11.6 
Table 11-7: $\quad$ Sample Property Set Declarations

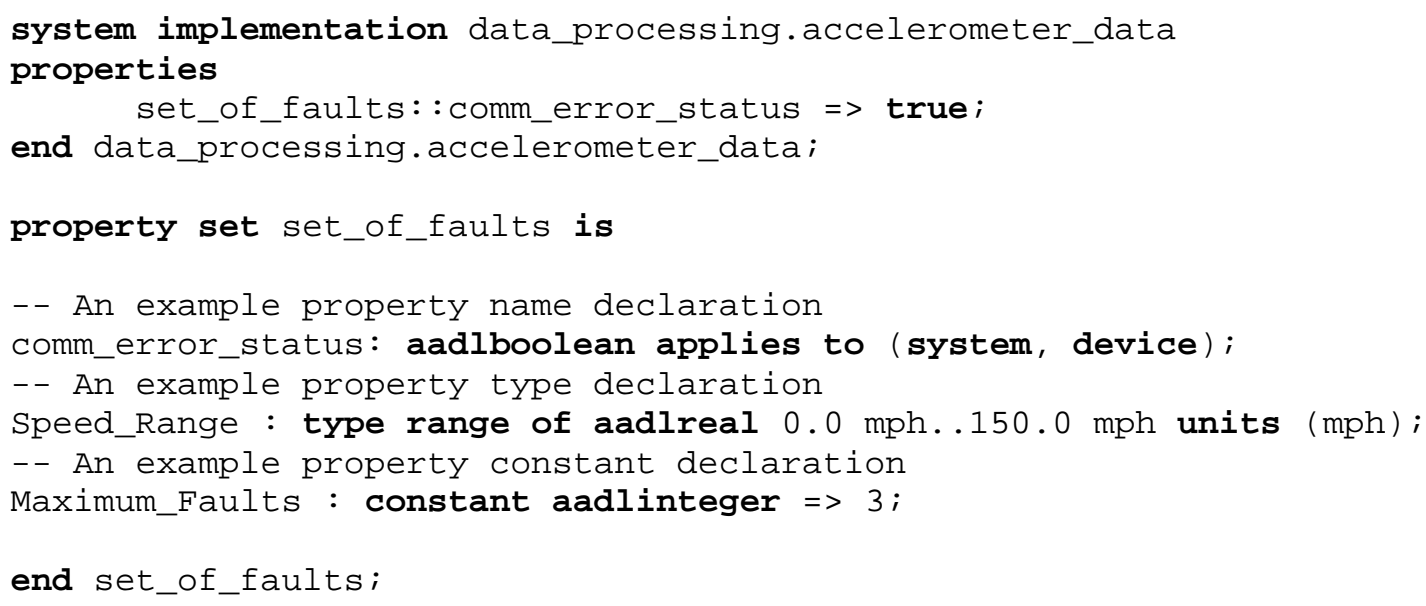

\subsection{Property Type Declarations}

A property type declaration defines a property type by associating an identifier with it and establishing the set of legal values for a property of that type. The declaration consists of

1. the desired identifier for the property type

2. a colon (:)

3. the reserved word type

4. an explicit type definition

5. a terminating semicolon (;)

The pattern for a property type declaration is shown in the box below:

identifier: type property type definition;

A property type definition may be an AADL built-in property type, a specialized type explicitly defined within the declaration, or a reference to previously defined property type.

In the examples shown in Table 11-8, the property type bit_error is defined as an aadlboolean property type. The predefined aadlboolean property type has two legal values, true and false. The property types fault_category and fault_condition are defined as enumeration types. An enumeration property type defines a specific set of identifiers as its legal values.

Type declarations can be more complex than simple base types. For example, the type number_of_components is declared in the property set more_types as an aadlinteger that ranges over the value 0 . . 25. The property boat_length is declared as a type of aadlreal with the units of feet that ranges over the values of 7.5 
.. 150.0 units ( feet ). The property voltage_ranges is a type of aadlreal that is a range of values that can span $0.0 \ldots 5.3$ units (volts).

Table 11-8: $\quad$ Sample Property Type Declarations

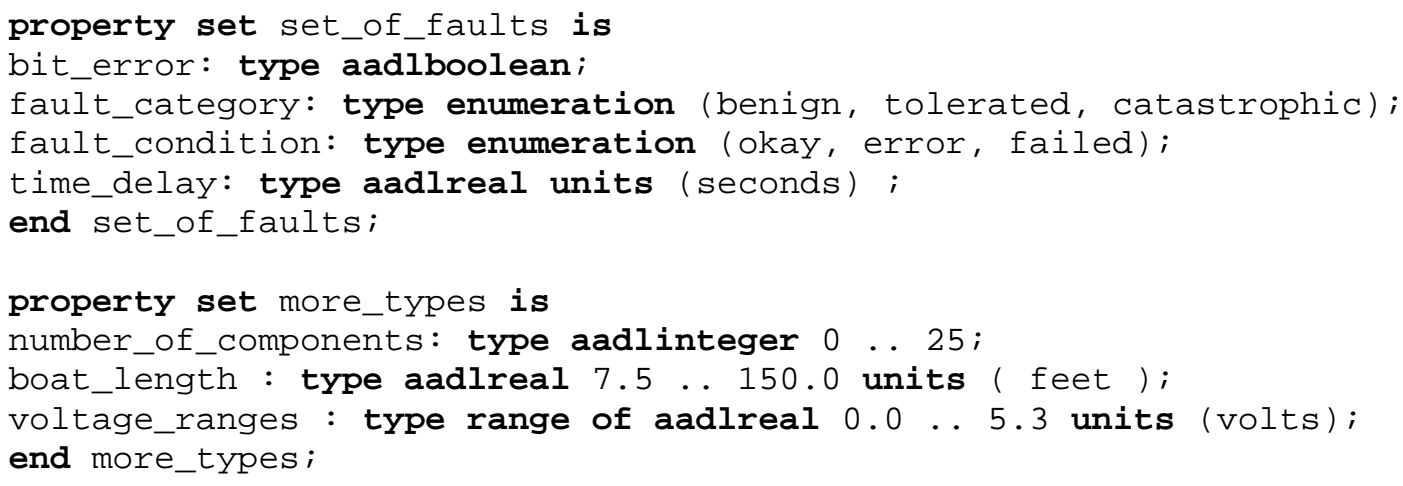

\subsection{Property Name Declarations}

A property name declaration defines a property by declaring a name, identifying a type for the property, and applying the property to a category of element within the specification (i.e., component, mode, port group, flow, port, server subprogram, or connection). A property name declaration consists of

1. desired identifier for the property name

2. $\operatorname{colon}(:)$

3. neither, either, or both of the reserved words (access or inherit)

4. explicit type identifier

5. reserved words (applies to)

6. property owner category or the reserved word (all)

7. terminating semicolon (;)

The pattern for a property name declaration is shown in the box below:

name : [access inherit property type applies to (property owner category);

A property owner category can be a component (e.g., system, thread, device), mode, port group, flow, port (event or data), server subprogram, parameter, or connections (port group, event port, data port, access, or parameter).

Example property name declarations within a property set set_of_names are shown in Table 11-9. Property name declarations can include the access and inherit options. A property declared with the reserved word inherit indicates that a value is inherited from a containing component, if a property value cannot be determined for a component. 
This inheritance can be seen in the declaration for the property critical_unit that is declared as inherit and as type aadlboolean and applies to all component categories. A property declared with the reserved word access is associated with access to a subcomponent rather than to the data component itself. The property queue_access is declared as a true-false access property for a data component. This can be used to restrict required access to a data queue. The property required_sensor_array_size is declared as type array that is declared within the property set set_of_types that is shown in the lower portion of Table 11-9.

Similarly, the property dangerous_voltages is declared with a type voltage_ranges that is declared in the property set more_types found in Table $11-8$.

Table 11-9: $\quad$ Sample Property Name Declarations

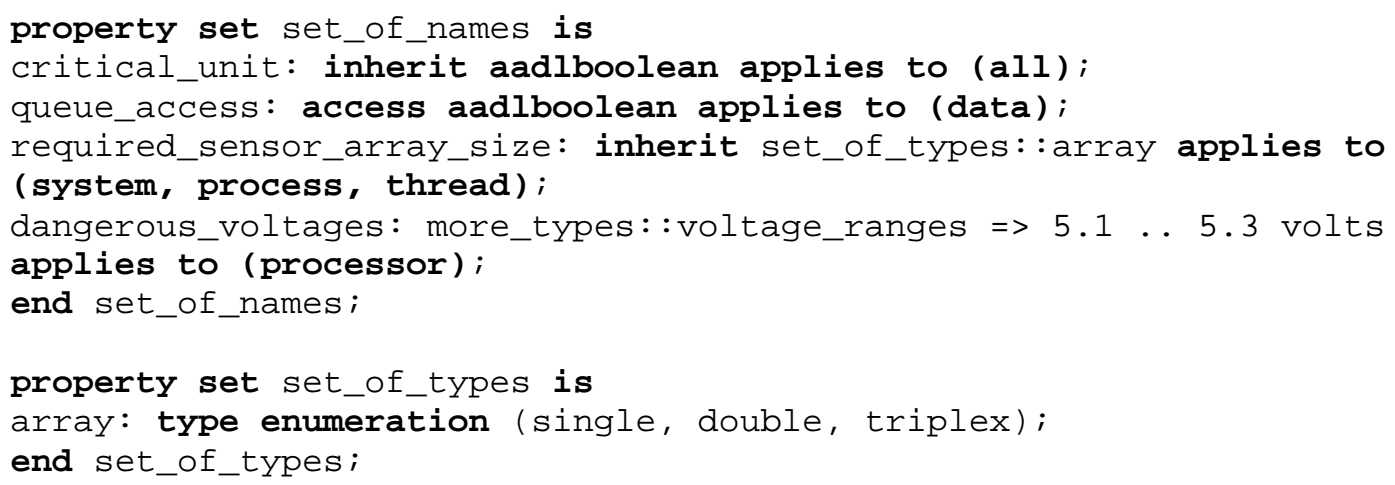

\subsection{Property Constant Declarations}

Property constants are property values that are known by a symbolic name. Property constants are provided in the predeclared property sets and can be defined in named property sets. They can be referenced in property expressions by name wherever the value itself is permissible.

Here are the basic declaration forms for a property constant declaration:

\begin{tabular}{|c|}
\hline identifier: constant (type) $=>$ property value \\
\hline identifier: constant list of $($ type $)=>$ property values \\
\hline
\end{tabular}

In the forms shown above

- Identifier is the name that can be used as a value in property associations.

- Entry (type) is a built-in type or a type declared in a property set.

- Property value or values must be of the type included in the constant declaration. 
Some sample declarations are shown in Table 11-10, where, for the property set limits_set,

- Max_Threads is defined as an integer value of 256.

- Minimum_value is defined as a real value of 5. $\odot$.

- Default_Fault_State is defined as a constant of the type fault_condition with the value of okay.

The type fault_condition, mentioned in Table 11-10, is defined in the package set_of_faults, as shown in Table 11-8.

Table 11-10: Sample Property Constant Declarations

property set limits_set is

Max_Threads : constant aadlinteger $=>256$;

Minimum_value: constant aadlreal $=>5.0$;

Default_Fault_State: constant set_of_faults: :fault_condition => okay;

end limits_set 


\section{Organizing a Specification}

This section presents language constructs that can be used to organize an AADL specification by grouping like elements using packages or design patterns.

\subsection{Packages}

A package is a named grouping of declarations and property associations that can be used to organize a specification. Packages establish distinct namespaces. However, they do not define an architectural hierarchy or design structure and cannot be declared inside other packages.

A package is divided into public and private segments. Declarations in the public segment are visible outside the package, whereas declarations in the private segment are visible only within the package. To reference an element in the public segment from outside a package, preface the element's identifier with the package name. In Table 12-1 for example, a process type compress_display_data contained in the public segment of the package display_dynamics_set would be referenced from outside the package as display_dynamics_set : : compress_display_data.

Also in Table 12-1, the specification for the system display_management references the compress_display_data process declared in the package display_dynamics_set. The data component new_format declared in the private segment of the package cannot be accessed from outside. However, the data component display_data can be, since it is declared in the public segment of the package. 
Table 12-1: Example Package Declaration

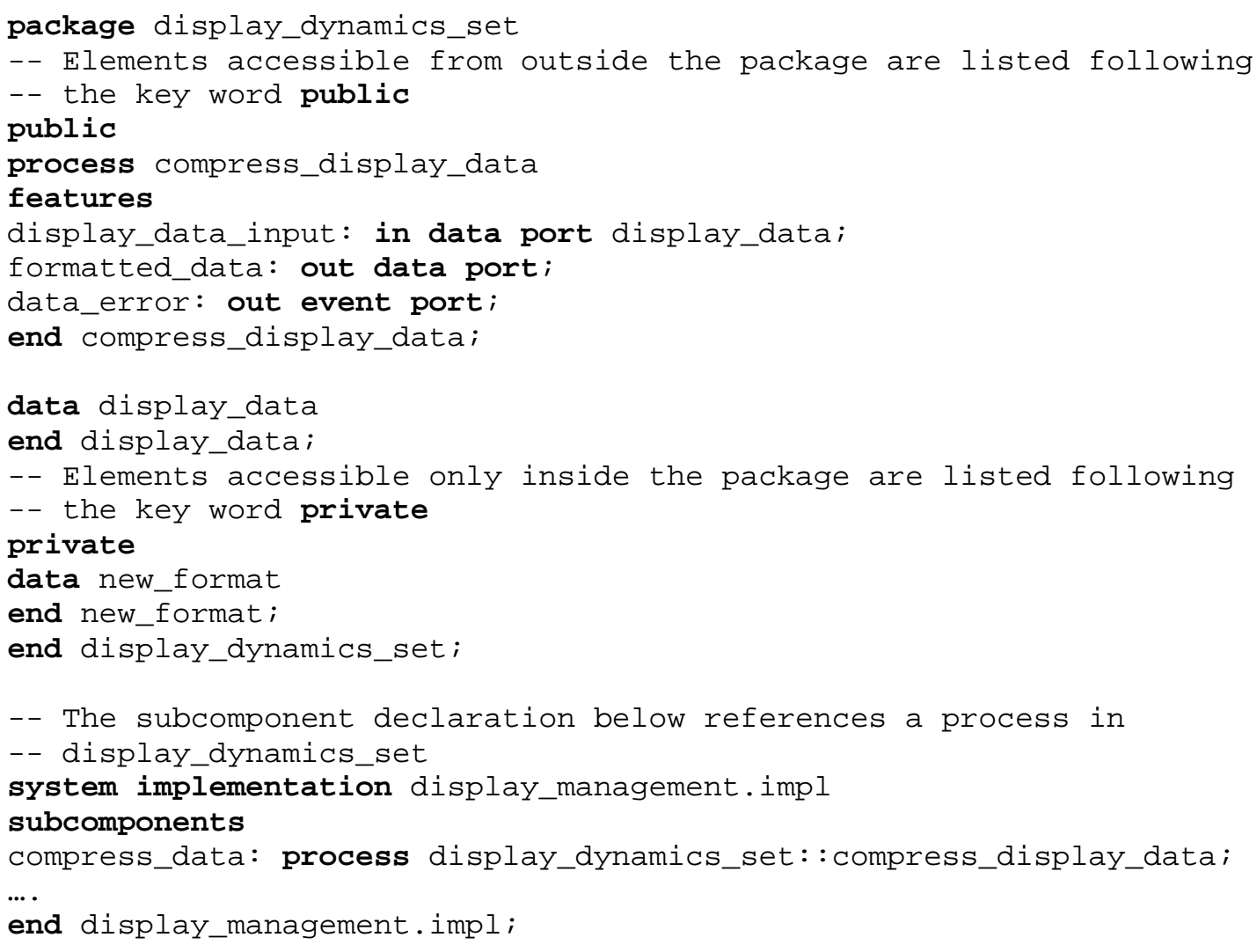

A package name can include multiple identifiers separated by a double colon (: : ). Thus, a package name like "primary_control_system::roll_axis::control_components" is permitted. This naming flexibility can be useful for packages that have been developed independently and have been assigned the same name. For example, consider two engineering teams working on a project, team red and team blue. Each team develops a package with the name "sensor_control." These packages can be renamed "team_red::sensor_control" and "team_blue::sensor_control". ${ }^{24}$ This would establish separate namespaces for each package and allow references to components with the same name within each package. That is, "team_red::sensor_control::controller" would reference a different declaration than "team_blue::sensor_control::controller." In addition, this flexibility can be used to associate packages logically. For example, two packages "roll_control" and "yaw_control" can be associated by renaming them "aircraft::roll_control" and "aircraft::yaw_control."

Packages can be used to organize layers of a design. For example, a package can be defined for a flight manager subsystem using constituent component subsystems, packages

24 The AADL standard states that "A defining package name must be unique in the global namespace. This means that the first identifier in a package name must be unique in the global namespace. Succeeding identifiers in the package name must be unique within the scope of the previous identifier" [SAE 06a]. 
that contain generic (common) descriptions, or packages containing only data types (e.g., a data dictionary). This concept is shown in the partial specification and packages of Table 12-2 where the Flight_Manager type declaration and declarations within the package avionics_subsystems reference components defined in separate packages.

In particular, in the portion of Table 12-2 labeled (1), the Flight_Manager component type declaration extends the Flight_Manager system type declared in the avionics_subsystems package. In the section labeled (2), the data type avionics_data: :raw_data, declared in the package avionics_data in the section labeled (4), is used in the avionics_subsystem package. And, in table section (3), the GPS subcomponent is an instance of the implementation GPS. impl from the avionics_sensor package. The comment lines (- - .....) indicate that other declarations required for a complete system specification are not shown.

Table 12-2: $\quad$ Example Design Organization Using Packages

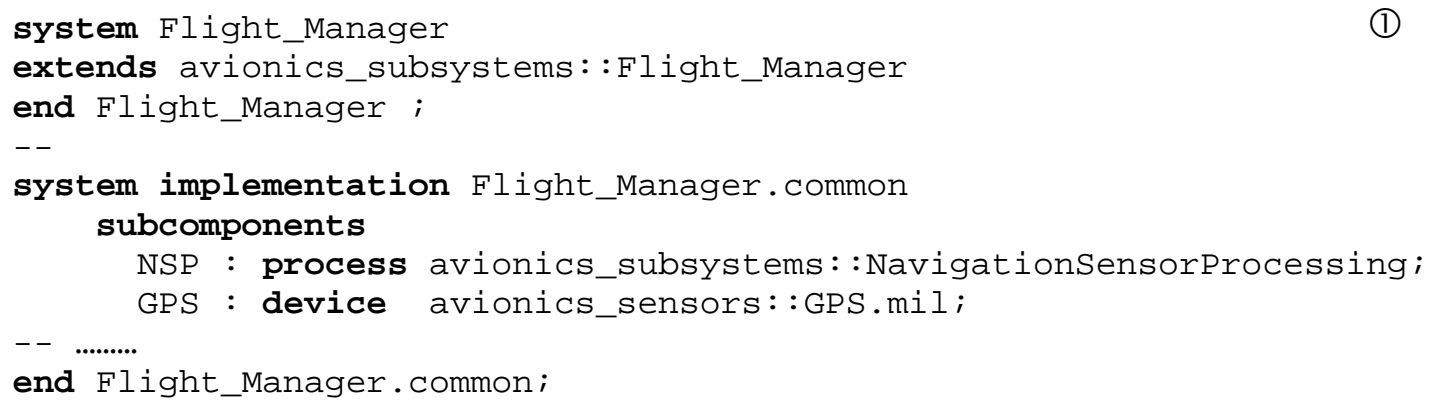


Table 12-2: Design Organization Using Packages (cont.)

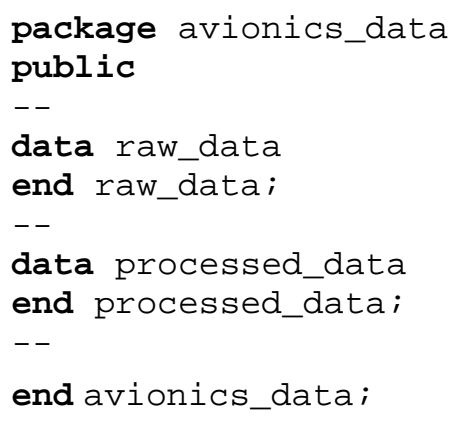

\subsection{Design Patterns}

A collection of specifications can be defined that form a set of extensible design patterns. Using AADL extension and refinement capabilities, these patterns can be used to develop specific application models.

\subsubsection{Type Extensions}

Elements of a design pattern set can involve core type declarations whose features are only partially defined. These core types as well as their descendents can be repeatedly extended, defining more specific types through feature refinements (refined to), as shown in Table 12-3. In that example, the core type one_dimensional_control is extended to form two specific types: (1) roll_control and (2) pitch_control. In these extensions, the partially defined in port and out port are refined to include specific data types. For the type declaration for roll_control, another input data port is added.

In general, new features can be added; partially defined features, completed; and property associations, added or modified. In the example in Table 12-3, the Required_Connection property value is changed in the roll_control extension. ${ }^{25}$ In the pitch_control extension, the Source_Name property association is added. The refinement options for type extension declarations are summarized on page 124 in the Appendix.

25 The default value for the predeclared property Required_Connection is true. However, it is declared explicitly as true in this example to demonstrate the refinement of property associations. 
Table 12-3: Example Type Extension

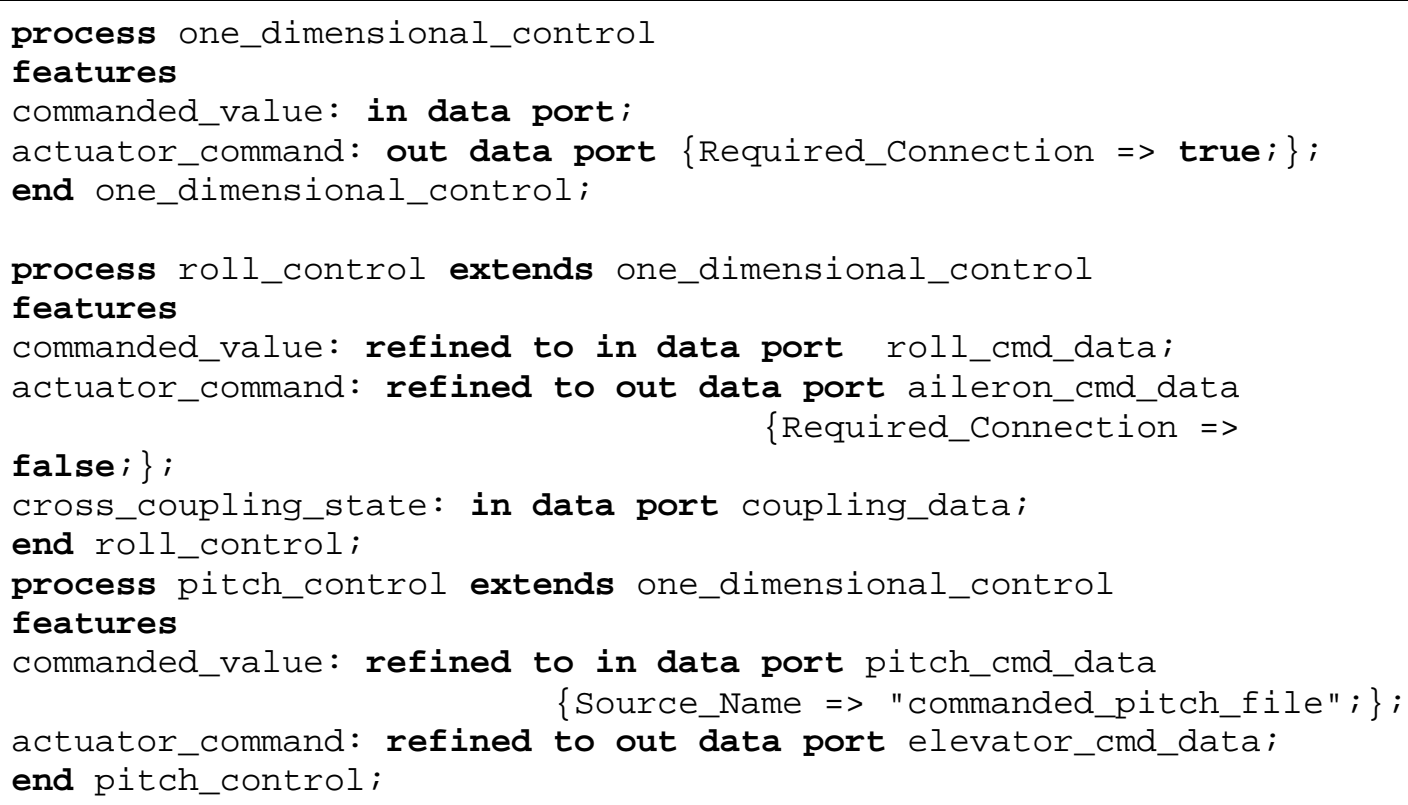

\subsubsection{Refinements within Implementations}

In an implementation declaration, the refines type subclause can be used to add or modify feature property associations of an implementation's type. For example, consider the server subprogram features for the thread type reader shown graphically and as AADL text in Table 12-4. There are two thread implementations, one for reading temperature (reader . temp) and one for reading pressure (reader . pressure). Each modifies the computation execution time value and adds a property association that defines a value for the subprogram's compute deadline. Note that including the name of the feature being refined (in this example a subprogram) in the refined to statement is optional. In the example, the subprogram read_data is included within the refined to declaration for the thread implementation reader . temp but is not included in the refined to declaration for the thread implementation reader.pressure. 
Table 12-4: Example Refines Type Implementation Subclauses
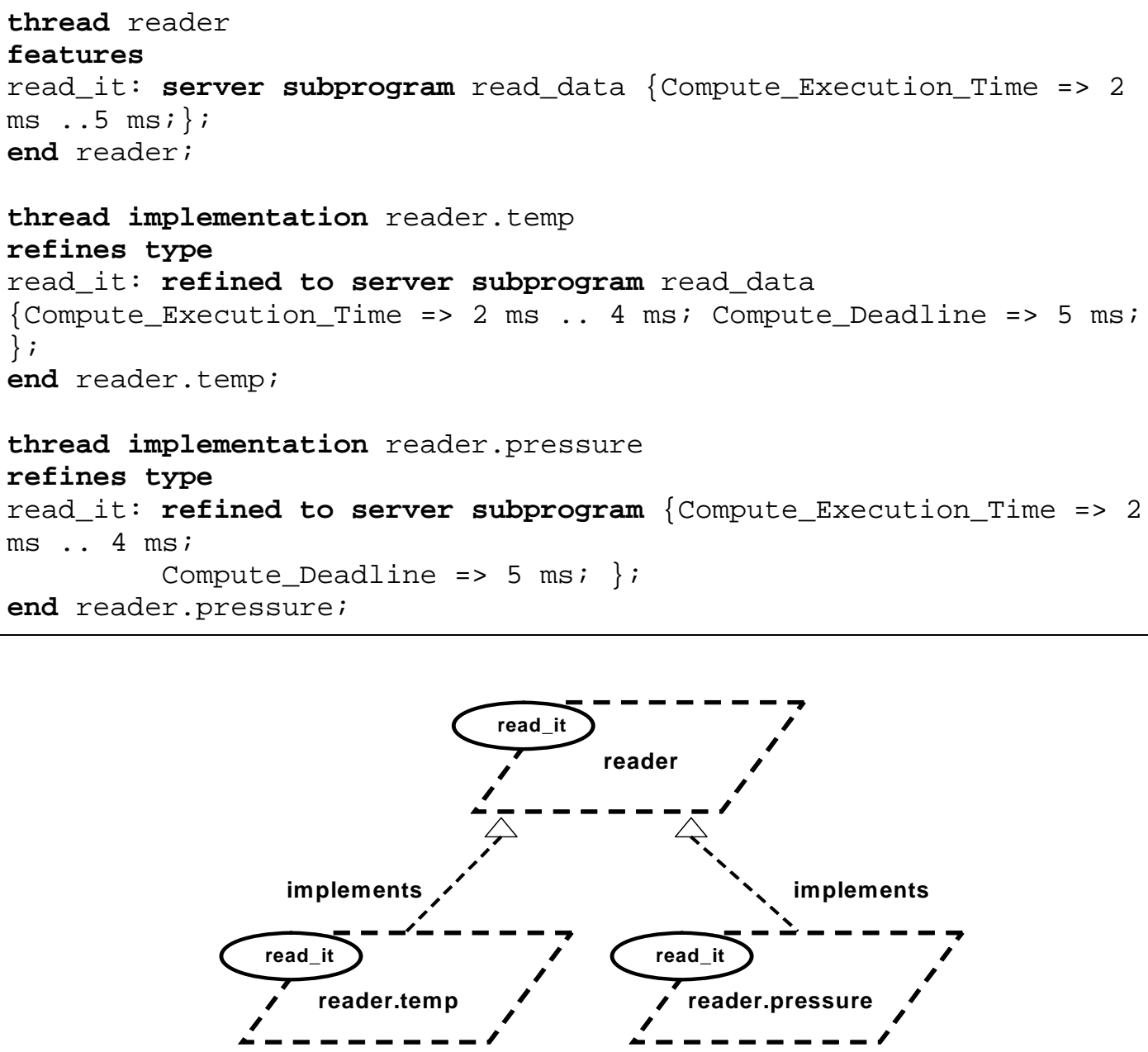

\subsubsection{Implementation Extensions}

Implementations can extend other implementations, modifying the underlying implementations and adding characteristics to them. Individual implementations can be extended multiple times, and extensions themselves can be extended. Implementation extensions can be integrated with type extension declarations to create an interrelated set of component types and implementations.

Table 12-5 shows example implementation extension declarations with accompanying type extension declarations for a flight control system. The type extension for flight_control_system adds an additional in data port sensor_set_redundant. Relationships among the declarations are shown graphically following the textual AADL specification. The refinement options for implementation extension declarations are summarized on page 125 in the Appendix. 
Table 12-5: Example Implementation Extensions

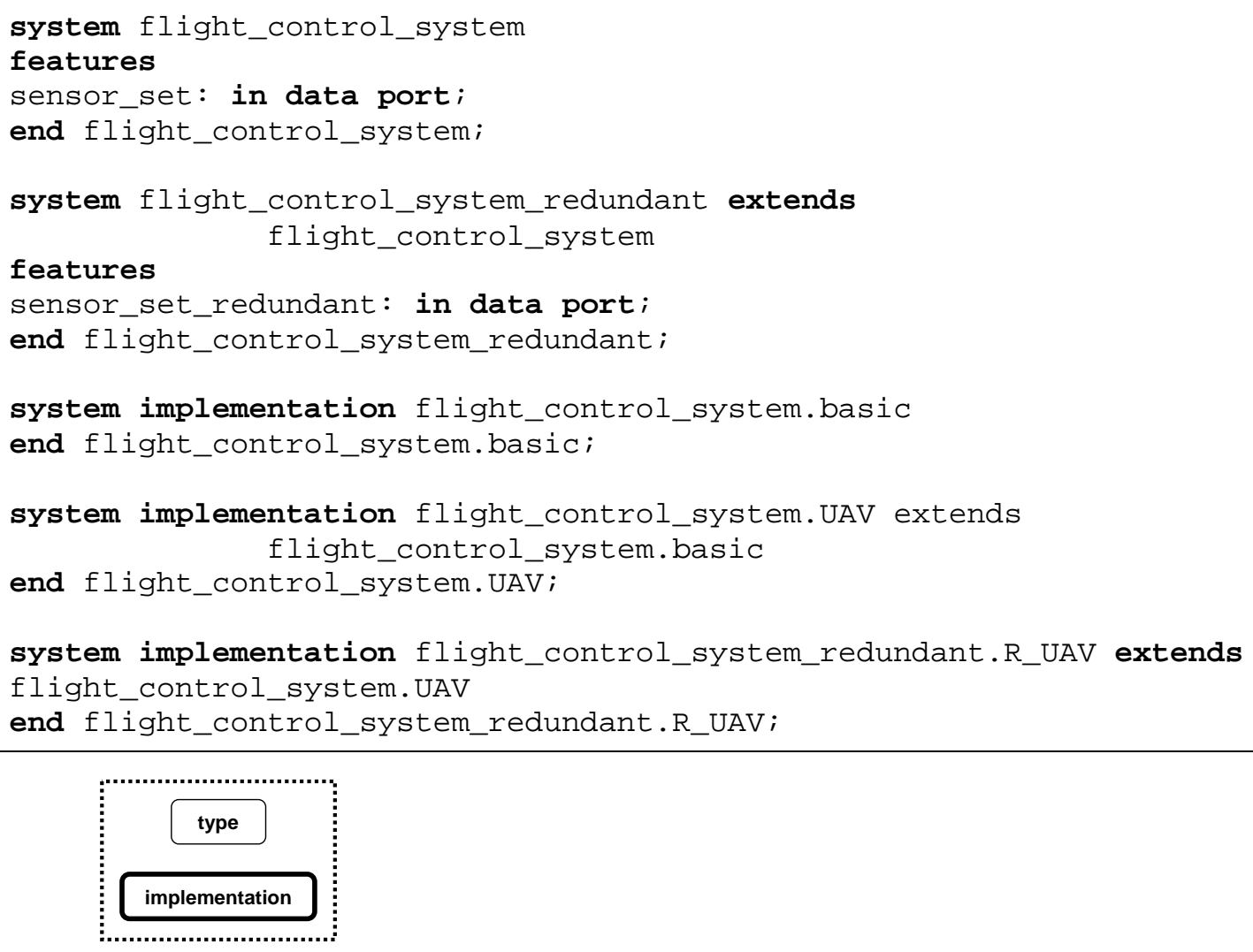




\subsubsection{Example Design Patterns}

In this section, the extension and refinement capabilities of the AADL are used to define a family of N-way Voting Lane components. Each $\mathrm{N}$-way component constitutes a lane within a redundant composite of $\mathrm{N}$-lanes and receives output data and system status opinions from the other lanes. Figure 12-1 shows a three-way lane system component.

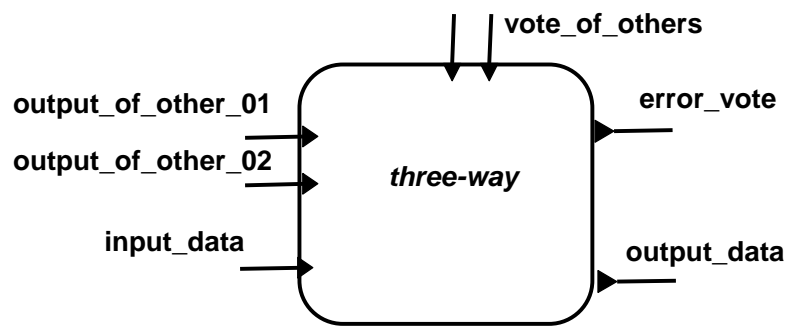

Figure 12-1: Three-way Voting Lane Component

The family of N-way Lane components depicted in Figure 12-2 is built upon extensions and refinements of generic type-implementation pairs. The core pair is a two-way voting generic type two-way and a generic implementation of that type two-way.g. The generic two-way voting type and implementation are extended to create a three-way voting generic type-implementation pair; the generic three-way voting type and implementation are extended to create a four-way voting generic typeimplementation pair.

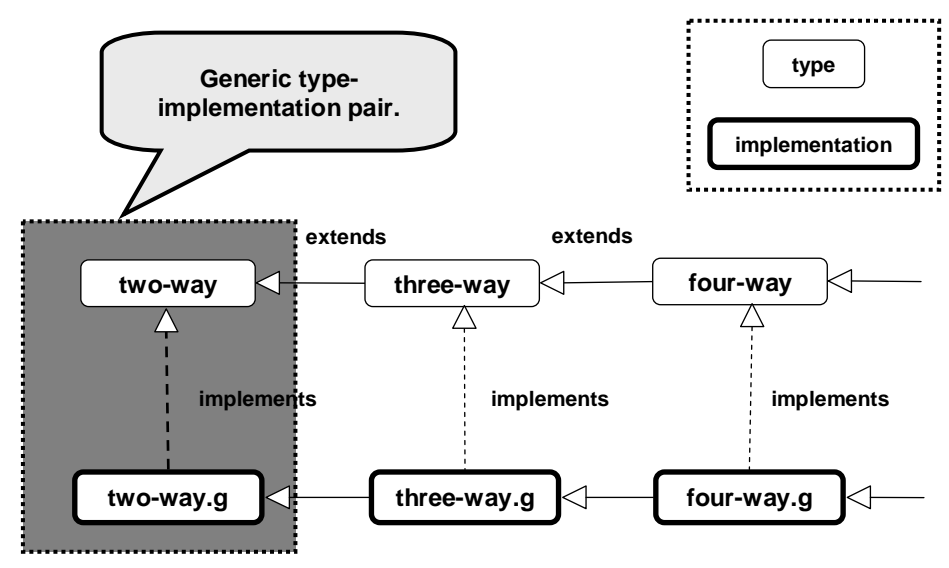

Figure 12-2: $\quad$ Generic N-way Voting Lanes Type-Implementation Pairs

Generic type-implementation pairs can be extensions along a well-defined aspect of the design. In this example of $\mathrm{N}$-way lane components, the aspect is the number of redundant lanes (voting ways) for the system. Generic implementations consist of general subclause declarations that can readily be refined in subsequent implementation extensions. In cascading generic implementations, partially defined subcomponents, calls, 
connections, flows, and modes are added. As appropriate, property associations are modified and added.

In cascading generic type declarations, features are partially defined, and basic property associations are declared. Generic type declarations consist of the following elements:

- partially defined features that can be completed in the refinements of a specialized extends type declarations

- basic flow declarations that can be used throughout the family with modifications only to the flow declaration property associations

- general property associations that characterize a component

In creating the family of type-implementation pairs illustrated in Figure 12-2, for instance, the two-way generic type is extended to create a three-way type by adding features that are partially defined rather than complete (e.g., data ports without data classifiers to handle the additional inputs from other lanes). The three-way generic implementation results from the extension of the two-way generic implementation. In this implementation extension, subcomponents, connections, modes, and other elements are added. Generic declarations should be sufficiently general to allow refinement by subsequent "voting" implementation extensions. The extension and refinement capabilities for types and implementations are summarized on pages 124-125 in the Appendix.

A specific realization of an aspect (e.g., a three-way system) is defined by an extension of the associated type-implementation pair, as shown in Figure 12-3. In the specific type extensions (extends), features are completed, features and flows are added, and relevant property associations are modified or added.

These declarations result in specialized realizations of the generic type. The specific implementation extensions (such as the three-way implementation generated from the three-way.g implementation in Figure 12-3) refine the general pattern of their associated generic implementations, providing all of the details required for instantiation. In the extension subcomponents definitions are completed; and calls, connections, flows, and modes are added.

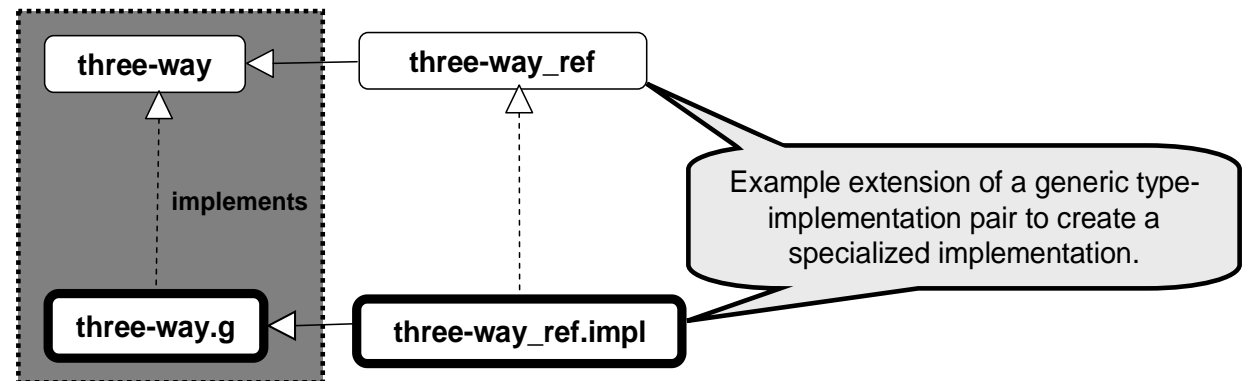

Figure 12-3: Specialized Extension and Refinement 


\section{Appendix}

\section{Component-Subcomponent Relationships}

Table 13-1 summarizes the permitted component-subcomponent relationships for each of the component abstractions in the AADL.

Table 13-1: $\quad$ Allowed Component-Subcomponent Relationships

\begin{tabular}{|c|c|c|c|}
\hline $\begin{array}{l}\text { Category } \\
\text { Group }\end{array}$ & $\begin{array}{l}\text { Component } \\
\text { Category }\end{array}$ & $\begin{array}{c}\text { Permitted } \\
\text { Subcomponents }\end{array}$ & $\begin{array}{c}\text { Permitted } \\
\text { Subcomponent of }\end{array}$ \\
\hline \multirow{5}{*}{ Software } & process & $\begin{array}{l}\text { thread } \\
\text { data } \\
\text { thread group }\end{array}$ & system \\
\hline & thread & data & $\begin{array}{l}\text { process } \\
\text { thread group }\end{array}$ \\
\hline & data & data & $\begin{array}{l}\text { process } \\
\text { thread } \\
\text { data } \\
\text { thread group } \\
\text { system }\end{array}$ \\
\hline & thread group & $\begin{array}{l}\text { data } \\
\text { thread } \\
\text { thread group }\end{array}$ & $\begin{array}{l}\text { process } \\
\text { thread group }\end{array}$ \\
\hline & subprogram & None allowed & None \\
\hline \multirow{4}{*}{$\begin{array}{l}\text { Execution } \\
\text { Platform }\end{array}$} & processor & memory & system \\
\hline & memory & memory & $\begin{array}{l}\text { processor } \\
\text { memory } \\
\text { system }\end{array}$ \\
\hline & bus & None allowed & system \\
\hline & device & None allowed & system \\
\hline Composite & system & $\begin{array}{l}\text { process } \\
\text { data } \\
\text { processor } \\
\text { memory } \\
\text { bus } \\
\text { device } \\
\text { system }\end{array}$ & system \\
\hline
\end{tabular}


Appendix

\section{Allowed Features}

Table 13-2 and Table 13-3 summarize the allowed features for each of the component abstractions in the AADL.

Table 13-2: $\quad$ Allowed Features for Components

\begin{tabular}{|c|c|c|}
\hline $\begin{array}{l}\text { Category } \\
\text { Group }\end{array}$ & $\begin{array}{l}\text { Component } \\
\text { Category }\end{array}$ & Allowed Features \\
\hline \multirow{5}{*}{ Software } & process & $\begin{array}{ll}\text { - } & \text { server subprogram } \\
\text { - } & \text { port/port group } \\
\text { - } & \text { provides data access } \\
\text { requires data access }\end{array}$ \\
\hline & thread & $\begin{array}{ll}\text { - } & \text { server subprogram } \\
\text { - } & \text { port/port group } \\
\text { - } & \text { provides data access } \\
\text { requires data access }\end{array}$ \\
\hline & data & $\begin{array}{l}\text { - } \quad \text { subprogram } \\
\text { - } \text { provides data access }\end{array}$ \\
\hline & thread group & $\begin{array}{ll}\text { - } & \text { server subprogram } \\
\text { - } & \text { port/port group } \\
\text { - } & \text { provides data access } \\
\text { requires data access }\end{array}$ \\
\hline & subprogram & $\begin{array}{ll}\text { - } & \text { out event port } \\
\text { - } & \text { out event data port } \\
\text { - } & \text { port group (event only) } \\
\text { - } & \text { requires data access } \\
\text { - } & \text { parameter }\end{array}$ \\
\hline \multirow{4}{*}{$\begin{array}{l}\text { Execution } \\
\text { Platform }\end{array}$} & processor & $\begin{array}{ll}\text { - } & \text { server subprogram } \\
\text { - } & \text { port/port group } \\
\text { - } & \text { requires bus access }\end{array}$ \\
\hline & memory & requires bus access \\
\hline & bus & requires bus access \\
\hline & device & $\begin{array}{l}\text { port/port group } \\
\text { - } \quad \text { server subprogram } \\
\text { - } \quad \text { requires bus access }\end{array}$ \\
\hline Composite & system & $\begin{array}{ll}\text { - } & \text { server subprogram } \\
\text { - } & \text { port/port group } \\
\text { - } & \text { provides data access } \\
\text { - } & \text { requides bus access } \\
\text { - } & \text { requires bus access } \\
\end{array}$ \\
\hline
\end{tabular}


Appendix

Table 13-3: Features and Allowed Components

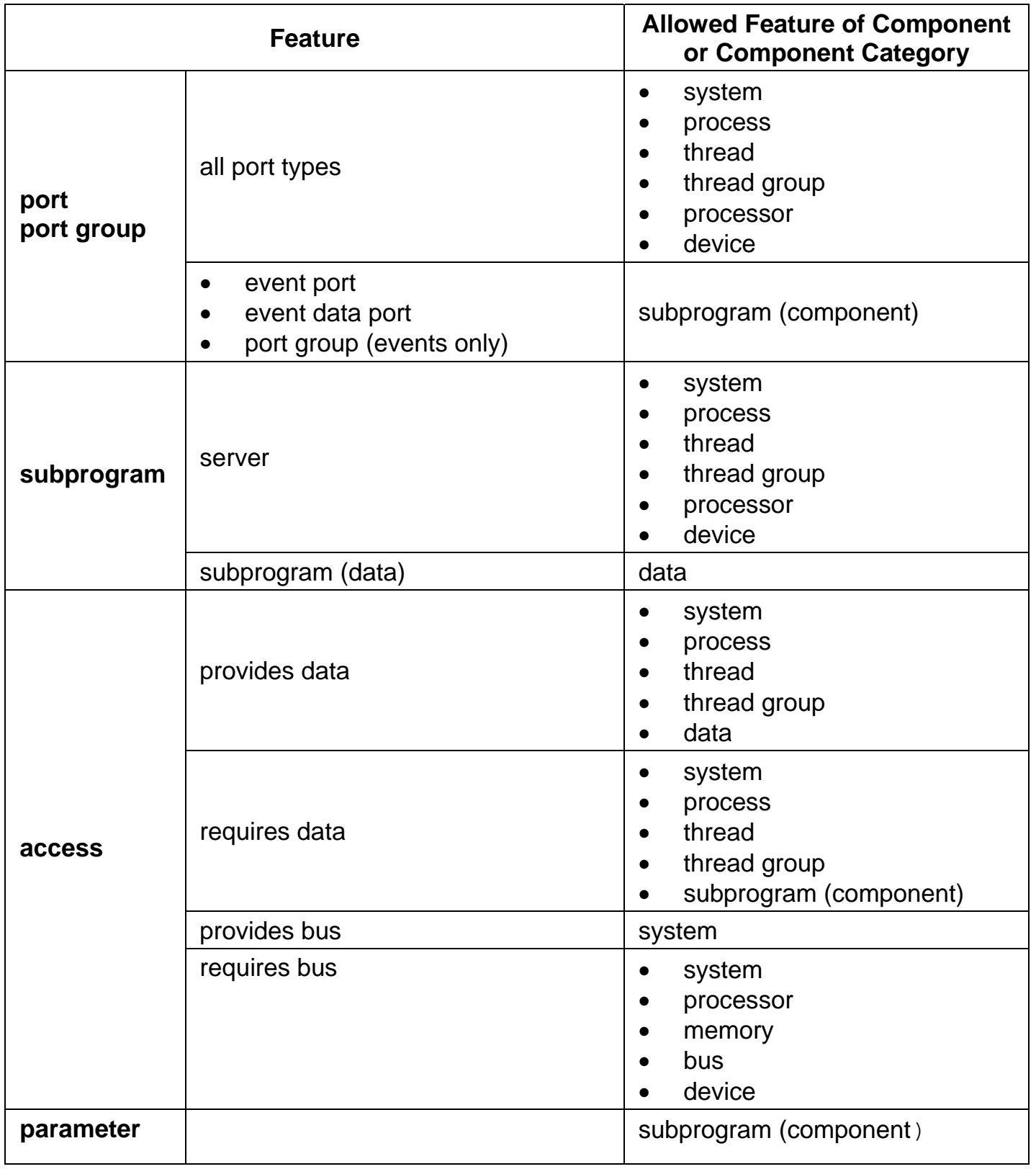


Appendix

\section{Constraints Summary}

Table 13-4 contains a summary of the legality rules for AADL components from Version 1.0 of the standard.

\section{Table 13-4: $\quad$ Constraints/Restrictions for Components}

\begin{tabular}{|c|c|c|}
\hline $\begin{array}{c}\text { Component } \\
\text { Category }\end{array}$ & Type & Implementation \\
\hline data & $\begin{array}{l}\text { Features: } \\
\text { - subprogram } \\
\text { - provides data access } \\
\text { Flow specifications: no } \\
\text { Properties yes }\end{array}$ & $\begin{array}{l}\text { Subcomponents: } \\
\text { - data } \\
\text { Subprogram calls: no } \\
\text { Connections: access } \\
\text { Flows: no } \\
\text { Modes: yes } \\
\text { Properties yes }\end{array}$ \\
\hline subprogram & $\begin{array}{l}\text { Features: } \\
\text { - } \quad \text { out event port } \\
\text { - } \quad \text { out event data port } \\
\text { - } \quad \text { port group } \\
\text { - } \quad \text { requires data access } \\
\text { - parameter } \\
\text { Flow specifications: yes } \\
\text { Properties yes }\end{array}$ & $\begin{array}{l}\text { Subcomponents: } \\
\text { • none } \\
\text { Subprogram calls: yes } \\
\text { Connections: yes } \\
\text { Flows: yes } \\
\text { Modes: yes } \\
\text { Properties yes }\end{array}$ \\
\hline thread & $\begin{array}{l}\text { Features: } \\
\text { - } \quad \text { server subprogram } \\
\text { - } \quad \text { port } \\
\text { - } \quad \text { provides data access } \\
\text { - } \quad \text { requires data access } \\
\text { Flow specifications: yes } \\
\text { Properties yes }\end{array}$ & $\begin{array}{l}\text { Subcomponents: } \\
\text { - data } \\
\text { Subprogram calls: yes } \\
\text { Connections: yes } \\
\text { Flows: yes } \\
\text { Modes: yes } \\
\text { Properties yes }\end{array}$ \\
\hline thread group & $\begin{array}{l}\text { Features: } \\
\text { - } \quad \text { server subprogram } \\
\text { - } \quad \text { port } \\
\text { - } \quad \text { provides data access } \\
\text { - } \text { requires data access } \\
\text { Flow specifications: yes } \\
\text { Properties yes }\end{array}$ & $\begin{array}{l}\text { Subcomponents: } \\
\text { - data } \\
\text { - thread } \\
\text { - thread group } \\
\text { Subprogram calls: no } \\
\text { Connections: yes } \\
\text { Flows: yes } \\
\text { Modes: yes } \\
\text { Properties yes }\end{array}$ \\
\hline process & $\begin{array}{l}\text { Features: } \\
\text { - } \quad \text { server subprogram } \\
\text { - } \quad \text { port } \\
\text { - } \quad \text { provides data access } \\
\text { - } \quad \text { requires data access } \\
\text { Flow specifications: yes } \\
\text { Properties yes }\end{array}$ & $\begin{array}{l}\text { Subcomponents: } \\
\text { - data } \\
\text { - thread } \\
\text { - thread group } \\
\text { Subprogram calls: no } \\
\text { Connections: yes } \\
\text { Flows: yes } \\
\text { Modes: yes } \\
\text { Properties yes }\end{array}$ \\
\hline
\end{tabular}


Appendix

Table 13-4: $\quad$ Constraints/Restrictions for Components (cont.)

\begin{tabular}{|c|c|c|}
\hline $\begin{array}{l}\text { Component } \\
\text { Category }\end{array}$ & Type & Implementation \\
\hline processor & $\begin{array}{l}\text { Features: } \\
\text { - } \quad \text { server subprogram } \\
\text { - } \quad \text { port/port group } \\
\text { - } \quad \text { requires bus access } \\
\text { Flow specifications: yes } \\
\text { Properties yes }\end{array}$ & $\begin{array}{l}\text { Subcomponents: } \\
\text { - memory } \\
\text { Subprogram calls: no } \\
\text { Connections: no } \\
\text { Flows: yes } \\
\text { Modes: yes } \\
\text { Properties: yes }\end{array}$ \\
\hline memory & $\begin{array}{l}\text { Features } \\
\text { - requires bus access } \\
\text { Flow specifications: no } \\
\text { Properties yes }\end{array}$ & $\begin{array}{l}\text { Subcomponents: } \\
\text { - memory } \\
\text { Subprogram calls: no } \\
\text { Connections: no } \\
\text { Flows: no } \\
\text { Modes: yes } \\
\text { Properties yes }\end{array}$ \\
\hline bus & $\begin{array}{l}\text { Features } \\
\text { - requires bus access } \\
\text { Flow specifications: no } \\
\text { Properties yes }\end{array}$ & $\begin{array}{l}\text { Subcomponents: } \\
\text { - none } \\
\text { Subprogram calls: no } \\
\text { Connections: no } \\
\text { Flows: no } \\
\text { Modes: yes } \\
\text { Properties yes }\end{array}$ \\
\hline device & $\begin{array}{l}\text { Features } \\
\text { - } \quad \text { port/port group } \\
\text { - } \quad \text { server subprogram } \\
\text { - } \quad \text { requires bus access } \\
\text { Flow specifications: yes } \\
\text { Properties yes }\end{array}$ & $\begin{array}{l}\text { Subcomponents: } \\
\text { - none } \\
\text { Subprogram calls: no } \\
\text { Connections: no } \\
\text { Flows: yes } \\
\text { Modes: yes } \\
\text { Properties yes }\end{array}$ \\
\hline system & $\begin{array}{l}\text { Features: } \\
\text { - } \quad \text { server subprogram } \\
\text { - } \quad \text { port/port group } \\
\text { - } \quad \text { provides data access } \\
\text { - } \quad \text { provides bus access } \\
\text { - } \quad \text { requires data access } \\
\text { - } \quad \text { requires bus access } \\
\text { Flow specifications: yes } \\
\text { Properties yes }\end{array}$ & $\begin{array}{l}\text { Subcomponents: } \\
\text { - data } \\
\text { - process } \\
\text { - } \text { processor } \\
\text { - } \text { memory } \\
\text { - device } \\
\text { - system } \\
\text { Subprogram calls: no } \\
\text { Connections: yes } \\
\text { Flows: yes } \\
\text { Modes: yes } \\
\text { Properties yes }\end{array}$ \\
\hline
\end{tabular}


Appendix

\section{Built-in Property Types}

Table 13-5 summarizes the AADL standard built-in property types.

Table 13-5: $\quad$ AADL Built-in Property Types

\begin{tabular}{|c|c|}
\hline Property Type & Definition \\
\hline aadlboolean & Two values, true or false \\
\hline aadlstring & All legal strings of the AADL \\
\hline enumeration & $\begin{array}{l}\text { An explicitly listed set of enumeration identifiers as } \\
\text { the set of legal values }\end{array}$ \\
\hline units & $\begin{array}{l}\text { An explicitly listed set of measurement unit identifiers } \\
\text { as the set of legal values }\end{array}$ \\
\hline aadlreal & A real value or a real value and its measurement unit \\
\hline aadlinteger & $\begin{array}{l}\text { An integer value or an integer value and its } \\
\text { measurement unit }\end{array}$ \\
\hline range & $\begin{array}{l}\text { Closed intervals of numbers indicating that a } \\
\text { property of this type has a value that is itself a range } \\
\text { term and specifies the number type of values in the } \\
\text { range term }\end{array}$ \\
\hline classifier & $\begin{array}{l}\text { Subset of syntactically legal component classifier } \\
\text { references whose category matches one of } \\
\text { component categories in the specified list }\end{array}$ \\
\hline reference & $\begin{array}{l}\text { Subset of syntactically legal references to those } \\
\text { components, whose category matches one of } \\
\text { component categories in the specified list, or to } \\
\text { connections or to server subprogram features; } \\
\text { indicated by the reserved word reference }\end{array}$ \\
\hline
\end{tabular}


Appendix

\section{AADL Reserved Words}

Table 13-6 lists the AADL reserved words. Reserved words are case insensitive.

Table 13-6: $\quad$ AADL Reserved Words

\begin{tabular}{|l|l|l|l|}
\hline aadlboolean & end & modes & reference \\
\hline aadlinteger & enumeration & none & refined \\
\hline aadlreal & event & not & refines \\
\hline aadlstring & extends & of & requires \\
\hline access & false & or & server \\
\hline all & features & out & set \\
\hline and & flow & package & sink \\
\hline annex & flows & parameter & source \\
\hline applies & group & path & subcomponents \\
\hline binding & implementation & port & subprogram \\
\hline bus & In & private & system \\
\hline calls & inherit & process & thread \\
\hline classifier & initial & processor & to \\
\hline connections & inverse & properties & true \\
\hline constant & Is & property & type \\
\hline data & list & provides & units \\
\hline delta & memory & public & value \\
\hline device & mode & range & \\
\hline
\end{tabular}




\section{Refinements within Type Extensions}

Table 13-7 summarizes the refinement capabilities within type extension declarations.

Table 13-7: $\quad$ Type Extensions and Associated Refinements

\begin{tabular}{|c|c|c|c|}
\hline \multicolumn{4}{|c|}{ Refinements within Type Extensions } \\
\hline Subclause & Refin & ent & Description (refined to) \\
\hline \multirow{6}{*}{ features } & ports & $\begin{array}{l}\text { data } \\
\text { event } \\
\text { data }\end{array}$ & $\begin{array}{l}\text { - } \quad \text { add ports (no refined to) } \\
\text { - complete partial declaration (add a data type or an } \\
\text { implementation classifier; change a data type } \\
\text { classifier to a data implementation classifier) } \\
\text { - } \quad \text { redefine or add port property associations }\end{array}$ \\
\hline & & event & $\begin{array}{l}\text { - } \quad \text { add event ports (no refined to) } \\
\text { - } \quad \text { redefine or add event port property associations }\end{array}$ \\
\hline & \multicolumn{2}{|c|}{ port group } & $\begin{array}{l}\text { - } \quad \text { add port groups (no refined to) } \\
\text { - } \quad \text { reference partial declarations (add missing type } \\
\text { implementation classifier) } \\
\text { - } \quad \text { redefine or add port group property associations }\end{array}$ \\
\hline & \multicolumn{2}{|c|}{ subprogram } & $\begin{array}{l}\text { - add server or data subprogram features (no refined } \\
\text { to) } \\
\text { - complete partial declarations (change type classifier } \\
\text { to an implementation classifier; no changes of } \\
\text { subprogram type or implementation classifiers) } \\
\text { - redefine or add subprogram property associations }\end{array}$ \\
\hline & \multicolumn{2}{|c|}{ parameters } & $\begin{array}{l}\text { - } \quad \text { add parameters (no refined to) } \\
\text { - complete partial declaration (no change of } \\
\text { parameter classifier to type or implementation; } \\
\text { change a type classifier to implementation) } \\
\text { - } \quad \text { redefine or add parameters property associations }\end{array}$ \\
\hline & \multicolumn{2}{|c|}{$\begin{array}{l}\text { subcomponent } \\
\text { access }\end{array}$} & $\begin{array}{l}\text { - } \text { add subcomponent access features (no refined to) } \\
\text { complete partial declaration (no subcomponent } \\
\text { classifier to type or implementation; type classifier to } \\
\text { implementation) } \\
\text { - } \text { redefine or add subcomponent property } \\
\text { associations }\end{array}$ \\
\hline flows & & & $\begin{array}{l}\text { - } \quad \text { add flow specifications (no refined to) } \\
\text { - } \quad \text { redefine or add flow property associations }\end{array}$ \\
\hline properties & & & - redefine or add component property associations \\
\hline
\end{tabular}




\section{Refinements within Implementation Declarations}

Table 13-8 summarizes the refinements associated within standard implementation declarations and implementation declarations that extends another.

Table 13-8: Implementations Extensions and Associated Refinements

\begin{tabular}{|c|c|}
\hline \multicolumn{2}{|r|}{ Refinements within Implementation Extensions } \\
\hline Subclause & Refinement Description \\
\hline refines type & - redefine or add feature property associations \\
\hline subcomponents & $\begin{array}{l}\text { - } \quad \text { add subcomponents (no refined to) } \\
\text { - } \quad \text { modify in modes with a new set of mode references } \\
\text { - } \quad \text { redefine or add subcomponent property associations }\end{array}$ \\
\hline calls & $\begin{array}{l}\text { - } \quad \text { add calls or call sequences (no refined to) } \\
\text { - no modification of call sequences }\end{array}$ \\
\hline connections & $\begin{array}{l}\text { - } \quad \text { add connections (no refined to) } \\
\text { - modify "in modes" references } \\
\text { - } \quad \text { redefine or add connection property associations }\end{array}$ \\
\hline flows & $\begin{array}{l}\text { - } \quad \text { add flow specifications (no refined to) } \\
\text { - } \text { modify in modes with a new set of mode references or mode } \\
\text { - redefine or add flow implementation property associations }\end{array}$ \\
\hline modes & $\begin{array}{l}\text { - } \quad \text { add modes (no refined to) } \\
\text { - } \quad \text { redefine or add mode property associations }\end{array}$ \\
\hline properties & - redefine or add component property associations \\
\hline
\end{tabular}




\section{Index}

\section{A}

AADL reserved words. 123 aadlboolean $95,102-106,122-123$ aadlinteger

$95,102,104,122-123$ aadlreal .... $102,104,122-123$ aadlstring $102,122-123$

Access.

Aggregate Data Port

All (reserved word)

And (reserved word)

Annex

Application software

Data

Process.

$34-36,58-59,62,71-72,81-84$

Subprogram

23-26

Thread

Thread group

Applies

$.34,36,41,46,56,71-72,76,83-84,97,105,118-119,123-124$

105,123

123

$4,7,10,13,20,129$

B

Binding

$25,33,55,67,71,100-102$

Bus. $46-48,74-77,121$

C

Calls

$77-79,84-85$

Classifier. $10,17-19,32,36,37,102,122-125$

Component

$37,41,77-80$

$26-33,52,61$

Component type $8,9,10,12,16-17,19,21,32,35,36,50,56,57,90-93,115,117-119$ Connections ............................................................... 9-10, 12, 19, 23, 34, 50, 56-58, 60-72, 74-77 $7,8,12,16,17,91-92$

Delayed $.81,84,86-88,92,95-98,101,105,111-112,116,122-123,125$ Immediate.

... 62, 64-66 Constant $.62-63,65-66$

Contained Property $96,102-103,106-107,123$

D

Data $34-37,58-59,62,71-72,81-84,120$

Data port..... $58-59,71$

Declarations $79,97-100$

Delta $12-13,16-20,26,30,40,57-59,68,70,72,77,91-93,95-97,104-108,125$

Device

$48-51,121$

\section{E}

End

$9,18,21,45,66-67,78,91-93,101,123$

Enumeration $102,104,122-123$

Error $.28,57-58,129$ 
Index

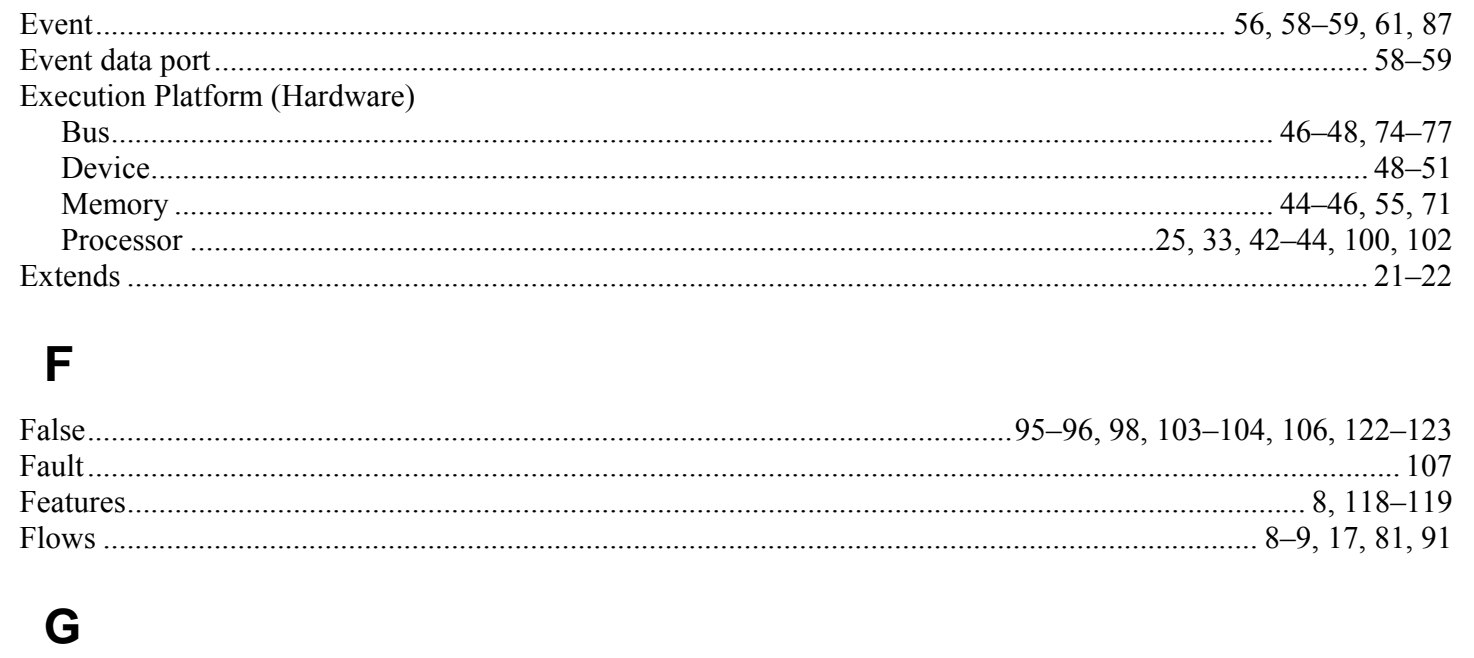

Group $1,4,8,10,12,14,19,23-25,30-33,37,41,50,52,54,60,67-71$, ..91, 96, 105, 117-119, 123-124

I

Identifier ... 106 Implementation $7,9,12,18-19,26,28,44,93,112-115,125$

In Inherit.... $9,100,105,123$ Initial Instance $32,86-87,123$

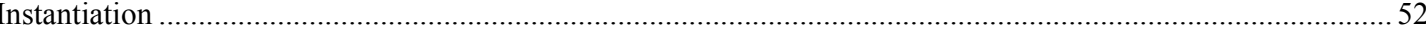

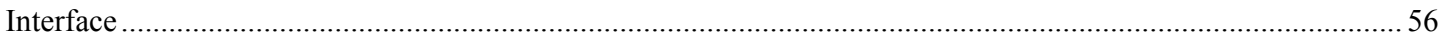

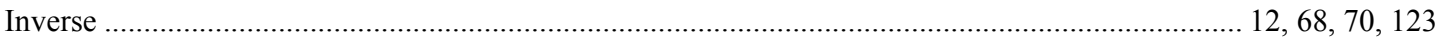

Is (reserved word)

\section{$\mathbf{L}$}

List......

\section{M}

Memory

$44-46,55,71,121$

Method calls

$2,9,78,86-89$

\section{$\mathbf{N}$}

Name 66-67, 105-106, 111

Namespace.

$10,19,21$

None

17-18, 123

Not

Of

Or

Out $14,18,24,28,41,57-58,61-64,67,70-71,81-82,84,87,91,98,111-112,118,123$

$\mathbf{P}$

Package

$12,19,109$ 


\section{Index}

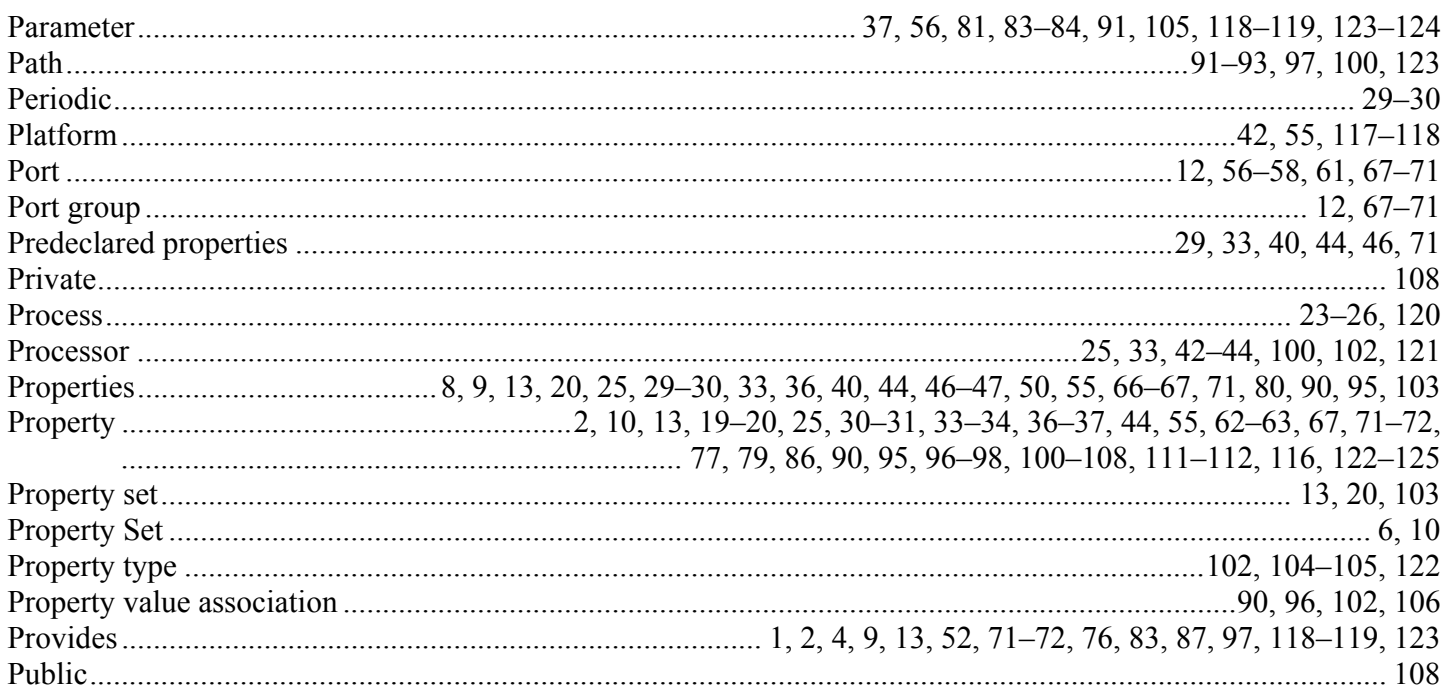

\section{$\mathbf{R}$}

Range $96,98,102-103,105,122-123$

Reference $14,31-32,34-35,67-68,79,81,83,100,102,104,108-110,122-124$

Refined $9,18,68,111-112,115,123-125$

Refines. $10,12,19,21,123,125$

Refines type 112

Remote calls

Requires

Reserved words.... $45,67,71-72,76,83,97,118-119,123$

\section{S}

Server.

Set.

Sink

Source

Subclause

Subcomponents

Subprogram

System

System Instance

$\mathbf{T}$

Thread Thread group

To

True

Type.
$23,39,52,55,79-80,96,105,112,118-119,122-124$ $1-2,12-13,20,24-25,33,55,58,67-68,71-72,87,95-97$, 102-104, 106-108, 111, 113, 122-123, 125 $91,93,123$ $4,23,25,28,33-34,36,38,43,50,52,58,60-62,66,71,91,93,123$ $30,124-125$ $4,9,10,12,14,19,23,32,34-38,41,45,52,60,69-71,76,79$, $87,90,92,95-98,100,115-116,123,125$ $. .37,41,77-80,120$ $5,11,50,52-54,60-61,73-74,121,129$ $54,60-61$

U

Units $. .5,10,46,102,104-105,122-123$

\section{V}

Value 102 


\section{References}

URLs are valid as of the publication date of this document.

[Feiler 04] Feiler, P. H.; Gluch, D. P.; Hudak, J. J.; \& Lewis, B. A. Embedded System Architecture Using SAE AADL (CMU/SEI-2004-TN-005). Pittsburgh, PA: Software Engineering Institute, Carnegie Mellon University, 2004. http://www.sei.cmu.edu/publications/documents /04.reports/04tn005.html

[SAE 06a]

Society of Automotive Engineers. SAE Standards: Architecture Analysis \& Design Language (AADL), AS5506, November 2004. http://www.sae.org/servlets/productDetail?PROD_TYP=STD\&PR OD_CD=AS5506 (2006)

[SAE 06b]

Society of Automotive Engineers. SAE Standards for Works in Progress: Error Model Annex, Draft version 0.91. http://www.sae.org/servlets/productDetail?PROD_TYP=STD\&PR OD_CD $=$ AS5506/2\&HIER_CD=TEAAS2\&WIP_SW=YES (2005)

[W3C 04] World Wide Web Consortium (W3C). Extensible Markup Language (XML) 1.0 (Third Edition). http://www.w3.org/TR/2004

/REC-xml-20040204/ (2004) 



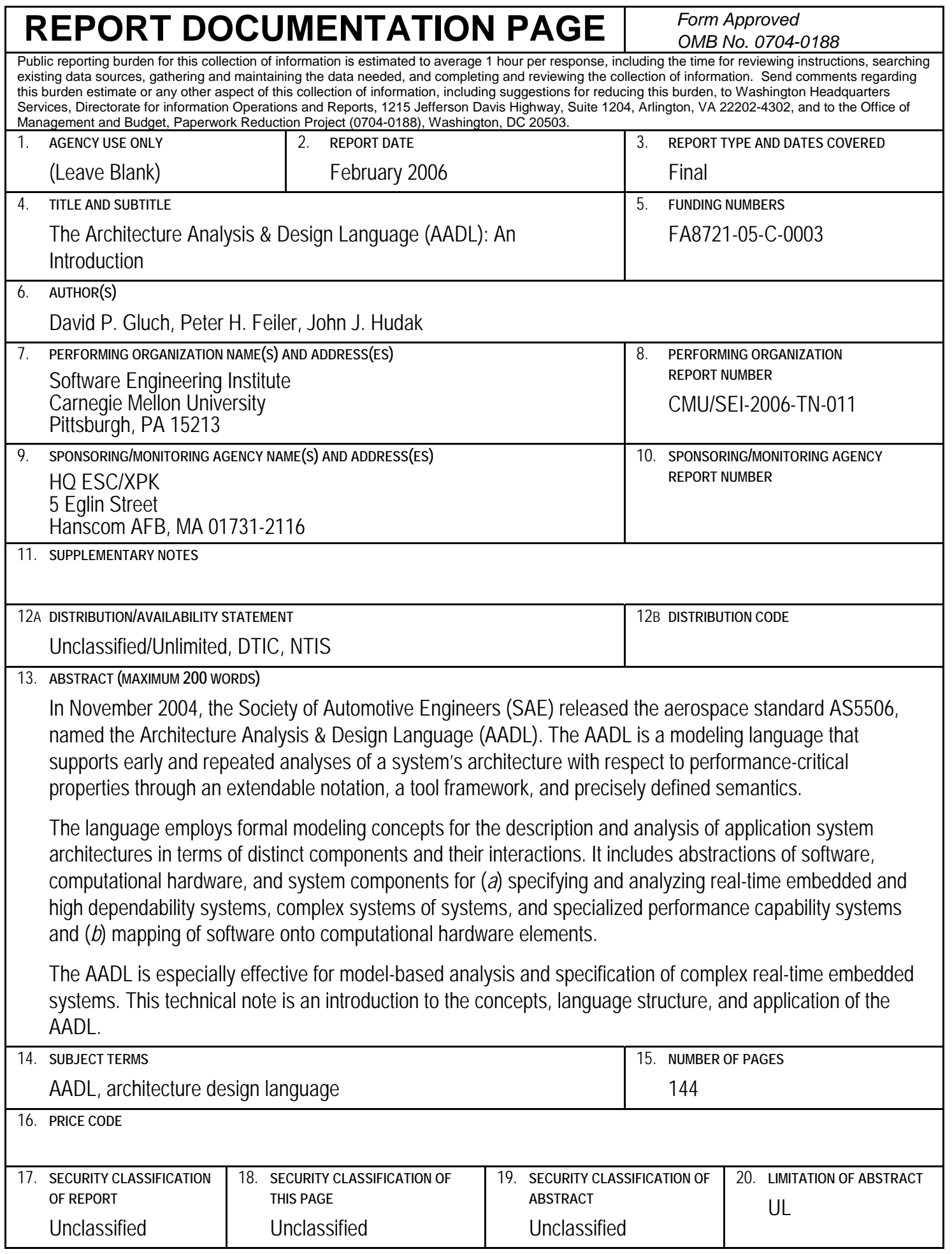

